\title{
Fahrerablenkung durch Informations- und Kommunikationssysteme, insbesondere Textbotschaften
}

Katja Schleinitz

Tibor Petzoldt

Josef Krems

Katharina Buchholz

Tina Gehlert 


\section{Fahrerablenkung durch Informations- und Kommunikationssysteme, insbesondere Textbotschaften}

Dr. rer. nat. Katja Schleinitz

Prof. Dr. Tibor Petzoldt

Prof. Dr. Josef Krems

M.A. Katharina Buchholz

Dr. rer. nat. Tina Gehlert

Unfallforschung der Versicherer 


\section{Impressum}

Gesamtverband der Deutschen Versicherungswirtschaft e. V.

Unfallforschung der Versicherer

Wilhelmstraße 43/43G, 10117 Berlin

Postfach 0802 64, 10002 Berlin

E-Mail: unfallforschung@gdv.de

Internet: www.udv.de

Facebook: www.facebook.com/unfallforschung

Twitter: @unfallforschung

YouTube: www.youtube.com/unfallforschung

ISBN-Nr.: 978-3-939163-79-4

Redaktion: Dr. rer. nat. Tina Gehlert

Bildnachweis: UDV und siehe Quellenangaben

Erschienen: 01/2018 


\section{Fahrerablenkung durch Informations- und Kommunikationssysteme, insbesondere Textbotschaften}

Bearbeitet durch:

Institut für Arbeits- , Organisations- und Verkehrspsychologie (IAOV GmbH)

in Kooperation mit TU Chemnitz Professur für Allgemeine und Arbeitspsychologie

Dr. rer. nat. Katja Schleinitz Prof. Dr. Tibor Petzoldt* Prof. Dr. Josef Krems

*inzwischen an der TU Dresden, Professur für Verkehrspsychologie

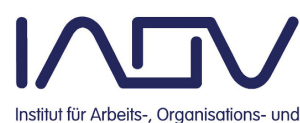
Institut für Arbeits-, Organisations- und
Verkehrsforschung GmbH Chemnitz
․ㅜㅂㅜ

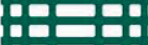

므를

TECHNISCHE UNIVERSITÄT

CHEMNITZ

Bei der UDV betreut von:

M.A. Katharina Buchholz

Dr. rer. nat. Tina Gehlert

Unfallforschung der Versicherer 



\section{Inhalt}

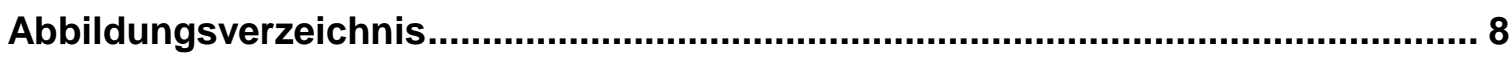

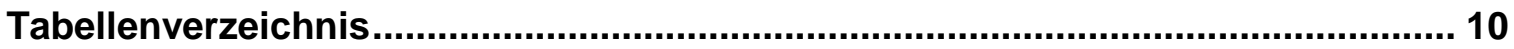

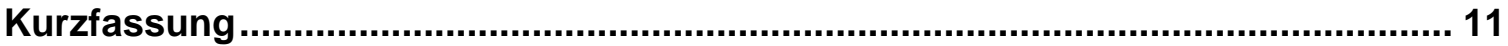

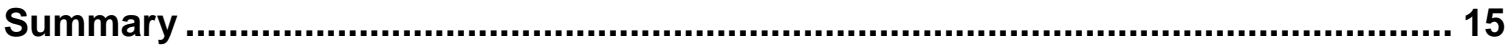

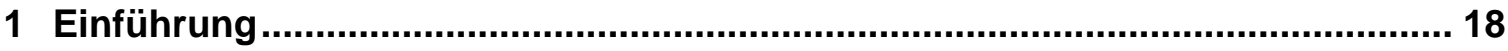

1.1 Auswirkungen des Lesens und Schreibens von Textnachrichten auf die Verkehrssicherheit ................................................................ 18

1.2 Auswirkungen des sprachgesteuerten Abhörens und Verfassens von Textnachrichten auf die Verkehrssicherheit ...........................................20

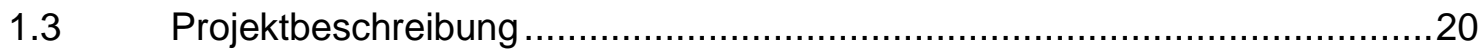

2 Literaturanalyse zu Kontexten der Fahrerablenkung ....................................... 22

3 Re-Analyse der SHRP2 Naturalistic Driving Study Daten .................................... 25

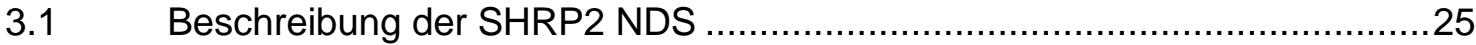

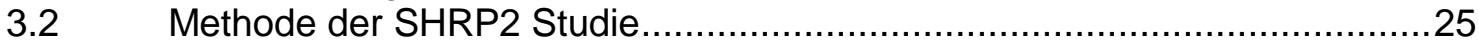

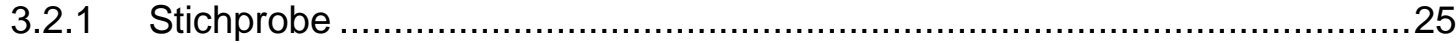

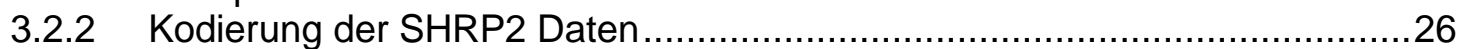

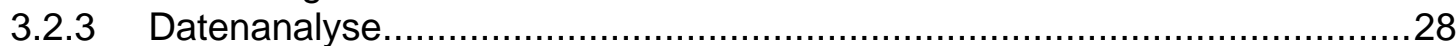

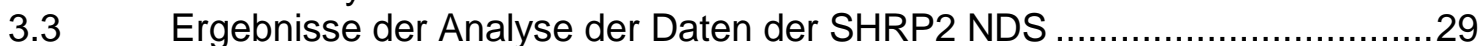

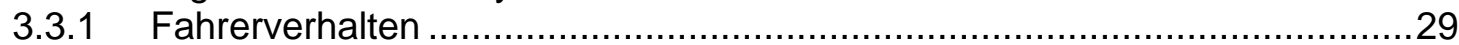

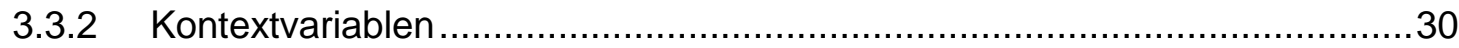

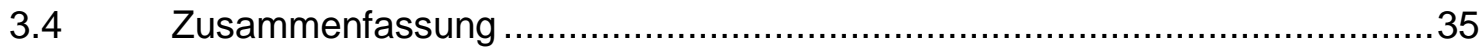

4 Videobasierte, strukturierte Interviewstudie zur Identifikation von Kontexten in denen Fahrer Textnachrichten verfassen ..................................... 37

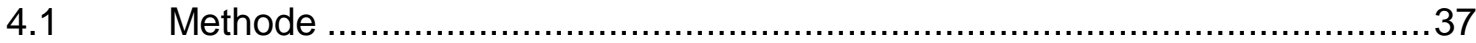

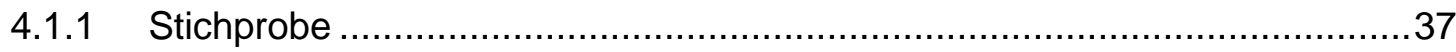

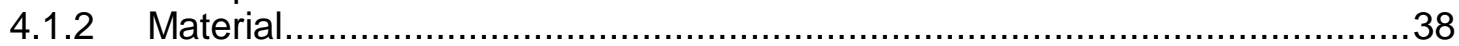

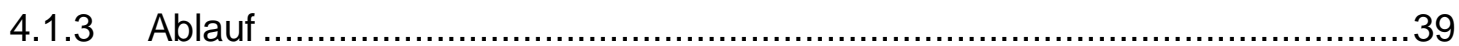

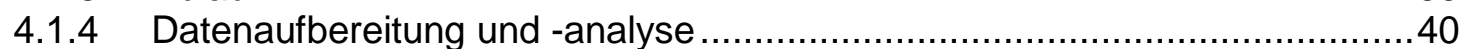

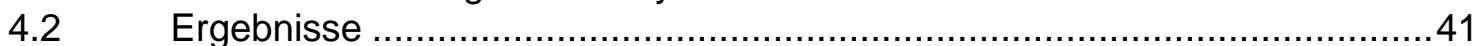

4.2.1 Häufigkeit des Lesens und Schreibens von Textnachrichten aus

Fragebogendaten ............................................................................ 41

4.2.2 Eingabestrategien bei der Eingabe einer Textnachricht ............................42

4.2.3 Häufigkeit des Verfassens von Textnachrichten in den gezeigten Verkehrssituationen .............................................................................43

4.2.4 Qualitative Aussagen zum Einfluss von Kontextvariablen auf das Verfassen von Textnachrichten ........................................................45

4.2.5 Einfluss von überdauernden (Persönlichkeits-)eigenschaften auf das Verfassen von Textnachrichten während des Fahrens........................49

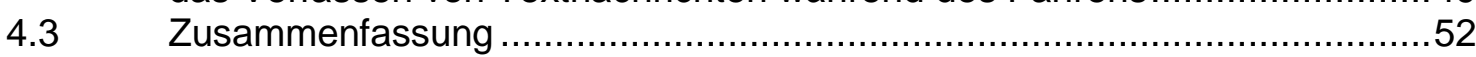

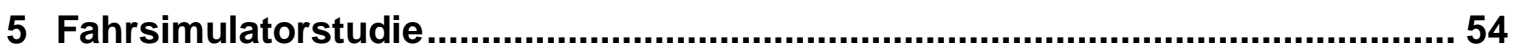

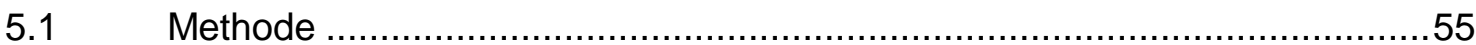

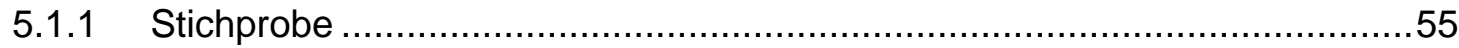

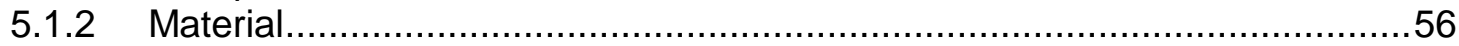

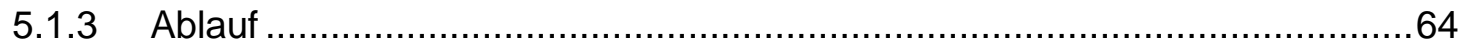

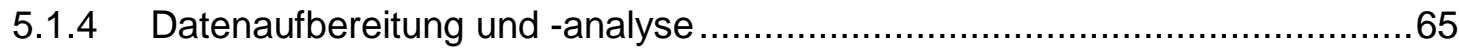




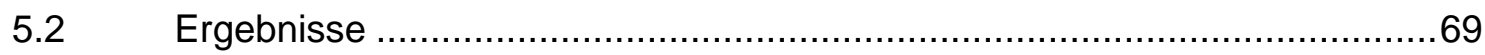

5.2.1 Häufigkeit des Lesens und Schreibens von Textnachrichten aus

Fragebogendaten ........................................................................ 70

5.2.2 Analyse von überdauernden (Persönlichkeits-)eigenschaften

bezüglich des Textens während des Fahrens .........................................71

5.2.3 Bearbeitung der Nebentätigkeit während der Fahrt im Simulator .................73

5.2.4 Vergleich Fahrperformanz auf geraden Streckenabschnitten mit und ohne Nebentätigkeit ..................................................................... 75

5.2.5 Besondere Verkehrssituationen - Kind auf der Straße ............................79

5.2.6 Besondere Verkehrssituationen - Baustelle ..............................................83

5.2.7 Besondere Verkehrssituationen - Schild $30 \mathrm{~km} / \mathrm{h}$ Geschwindigkeitsbegrenzung ........................................................ 86

5.2.8 Beanspruchung und der Einfluss von Kontextvariablen auf das Verfassen von Textnachrichten ..................................................... 87

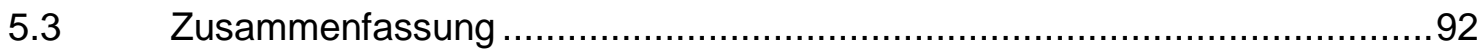

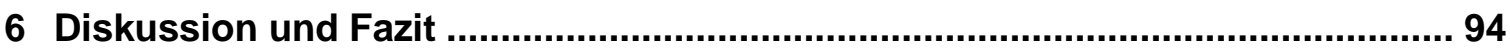

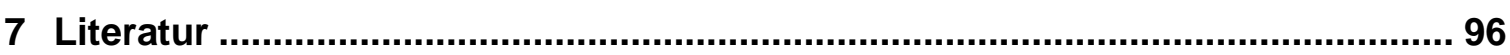

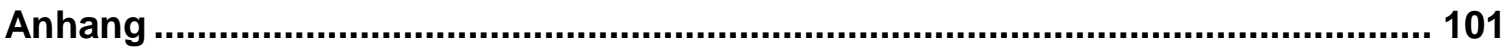




\section{Abbildungsverzeichnis}

Abbildung 1: verwendete Stichprobe des SHRP2 Datens atzes nach

Altersgruppen.

Abbildung 2: Videoausschnitt für eine Situation auf der Landstraße

Abbildung 3: Häufigkeit des Lesens von Textnachrichten bzw. der Nutzung Vorlesefunktion während des Fahrens, $n=40$.

Abbildung 4: Häufigkeit des Schreibens von Textnachrichten bzw. der Nutzung der Spracheingabe während des Fahrens, $n=40$.

Abbildung 5: Häufigkeit des Schreibens von Textnachrichten bzw. der Nutzung der Spracheingabe während des Fahrens $(N=41)$....

Abbildung 6: Häufigkeit der Anzahl der Situationen in denen die Teilnehmer eine Textnachricht schreiben würden $(N=41)$.

Abbildung 7: Links, eine Situation in der beinahe alle Teilnehmer eine Textnachricht verfasst hätten; rechts, eine Situation in der niemand eine Textnachricht verfasst hätte.

Abbildung 8: Zusammenhang zwischen Risikoerhöhung (in \%) und der Häufigkeit eine Textnachricht (in \%) zu schreiben $(N=41)$.

Abbildung 9: Nennungen (in \%) für Argumente warum Teilnehmer eine Textnachricht verfassen würden $(N=41)$.

Abbildung 10: Nennungen (in \%) für Argumente warum Teilnehmer keine Textnachricht verfassen würden $(N=41)$.

Abbildung 11: Nennungen (in \%) für Argumente was anders sein müsste, damit die Teilnehmer nicht mit dem Verfassen einer Textnachricht beginnen $(N=41)$.

Abbildung 12: Nennungen (in \%) für Argumente was anders sein müsste, damit die Teilnehmer mit dem Verfassen einer Textnachricht beginnen $(N=41)$.

Abbildung 13: Häufigkeit der Mittelwerte der Teilnehmer auf der Skala zur Einstellung gegenüber dem Schreiben von Textnachrichten während des Fahrens (Skala 1-7; $N=41$ ).

Abbildung 14: Häufigkeit der Mittelwerte der Teilnehmer auf der Skala zum gewohnheitsmäßigen Schreiben von Textnachrichten (Skala 1-7, $N=41$ ).

Abbildung 15: Mittelwerte der Zustimmung für die Skalen der Theorie des geplanten Verhaltens bezogen auf das Schreiben von Textnachrichten während des Fahrens $(N=41)$.

Abbildung 16: Überblick über Fahrsimulator inklusive Versuchsleiterarbeitsplatz............56

Abbildung 17: Ansicht integriertes System mit vier Kameras.

Abbildung 18: Ansicht Innenraum von „Rücksitz“ durch Innenraumkamera (links) und Ansicht der Strecke durch Szenenkamera aufgenommen (rechts).

Abbildung 19: Überblick über die Szenarien mit kritischen Situationen, oben mit Information als Vorinformation und unten ohne Information als Vorinformation, rechts Situationen mit dem überquerenden Kind, links mit der Baustelle.

Abbildung 20: Überblick über die Szenarien mit relevanten Verkehrsinformationen (Schild $30 \mathrm{~km} / \mathrm{h}$ Geschwindigkeitsbegrenzung).

Abbildung 21: Bearbeitung der Nebentätigkeit am Smartphone (links) bzw. am Touchdisplay (rechts)....

Abbildung 22: Überblick über die Datenquellen und Schritte zur Datenaufbereitung......65

Abbildung 23: Exemplarische Darstellung der Ermittlung der Intervalle für einen

Teilnehmer bei $50 \mathrm{~km} / \mathrm{h}$ Geschwindigkeitsbegrenzung.

Abbildung 24: Häufigkeit des Lesens von Textnachrichten bzw. der Nutzung

Vorlesefunktion während des Fahrens, Fragebogendaten $(N=82)$. 
Abbildung 25: Häufigkeit des Schreibens von Textnachrichten bzw. der Nutzung der Spracheingabe während des Fahrens, Fragebogendaten $(N=82) \ldots \ldots \ldots \ldots \ldots \ldots . . .70$

Abbildung 26: Häufigkeit des Schreibens von Textnachrichten bzw. der Nutzung der Spracheingabe während des Fahrens, Fragebogendaten $(N=82) \ldots \ldots \ldots \ldots \ldots \ldots . . . . .71$

Abbildung 27: Häufigkeit der Mittelwerte der Teilnehmer auf der Skala zur Einstellung gegenüber dem Schreiben von Textnachrichten während des Fahrens (links) und auf der zum gewohnheitsmäßigen Schreiben von Textnachrichten, Fragebogendaten (rechts, $N=82$ Personen).

Abbildung 28: Mittelwerte der Zustimmung für die Skalen der Theorie des geplanten Verhaltens bezogen auf das Schreiben von Textnachrichten während des Fahrens, Fragebogendaten ( $N=82$ Personen).

Abbildung 29: Dauer der Bearbeitung der Nebentätigkeit, Videodaten $(N=82)$

Abbildung 30: Durchschnittliche Geschwindigkeit Streckenabschnitt $30 \mathrm{~km} / \mathrm{h}$ Geschwindigkeitsbegrenzung, Fahrdaten ( $N=127$ Fahrten, 65 Teilnehmer, Fehlerbalken 95\% Konfidenzintervall).

Abbildung 31: Durchschnittliche Geschwindigkeit Streckenabschnitt 50 km/h Geschwindigkeitsbegrenzung, Fahrdaten ( $N=127$ Fahrten, 65 Teilnehmer, Fehlerbalken 95\% Konfidenzintervall).

Abbildung 32: Standardabweichung der lateralen Spurposition für Streckenabschnitt $30 \mathrm{~km} / \mathrm{h}$ Geschwindigkeitsbegrenzung, Fahrdaten ( $N=127$ Fahrten, 65 Teilnehmer, Fehlerbalken 95\% Konfidenzintervall)

Abbildung 33: Standardabweichung laterale Distanz für Streckenabschnitt $50 \mathrm{~km} / \mathrm{h}$ Geschwindigkeitsbegrenzung, Fahrdaten ( $N=127$ Fahrten, 65 Teilnehmer, Fehlerbalken 95\% Konfidenzintervall).

Abbildung 34: Reaktionszeit nach Sichtbarkeit des Balles bei Bremspedalbetätigung mit und ohne Vorinformation, Fahrdaten $(N=148$ Situationen, Fehlerbalken 95\% Konfidenzintervall).

Abbildung 35: Abstand zum Kind bei Bremspedalbetätigung mit und ohne Vorinformation, Fahrdaten ( $N=148$ Situationen, Fehlerbalken 95\% Konfidenzintervall).

Abbildung 36: links: Spearman Rho Korrelation zwischen Beanspruchung und Häufigkeit Texting $(N=6)$, rechts: Spearman Rho Korrelation zwischen Häufigkeit Texting und Risikoerhöhung, Interviewdaten $(N=6)$.

Abbildung 37: Nennungen (in \%) für Argumente warum Teilnehmer eine Textnachricht verfassen würden, Interviewdaten $(N=82)$.

Abbildung 38: Nennungen (in \%) für Argumente warum Teilnehmer keine Textnachricht verfassen würden, Interviewdaten $(N=82)$....

Abbildung 39: Nennungen (in \%) für Argumente was anders sein müsste, damit die Teilnehmer nicht mit dem Verfassen einer Textnachricht beginnen, Interviewdaten $(N=82)$.

Abbildung 40: Nennungen (in \%) für Argumente was anders sein müsste, damit die Teilnehmer mit dem Verfassen einer Textnachricht beginnen, Interviewdaten $(N=82)$. 


\section{Tabellenverzeichnis}

Tabelle 1: Überblick über Kontexte oder Umgebungsbedingungen und der

Bereitschaft Nebentätigkeiten nachzugehen. 23

Tabelle 2: Kreuztabelle zur Berechnung der Odds Ratio.............................................29

Tabelle 3: Fahrerverhalten: Fahrmanöver im betrachteten Abschnitt (Pre-incident behaviour)

Tabelle 4: Passagiere auf Vorder- und Rücksitz.

Tabelle 5: Umwelteinflüsse: Lichtverhältnisse, Witterungsbedingungen, Zustand

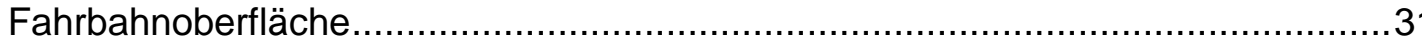

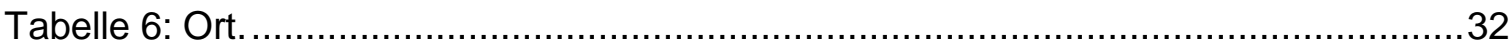

Tabelle 7: Infrastrukturmerkmale: Verkehrsführung, Fahrstreifen, Kreuzungstypen,

Verkehrsregelung, Baustellenbereich und Neigung. .............................................33

Tabelle 8: Fahrtmerkmale: Fahrtrichtung und Verkehrsdichte..................................34

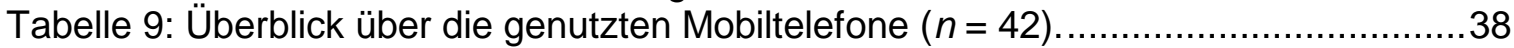

Tabelle 10: Prozent des Textens nach Komplexitätseinstufung nach Fastenmeier $(N=41)$

Tabelle 11: Prozent des Textens nach Straßentyp $(N=41)$.

Tabelle 12: Korrelationen der Skalen der Theorie des geplanten Verhaltens mit Anzahl der Situationen in denen Textnachricht verfasst worden wären $(N=41) \ldots . .51$

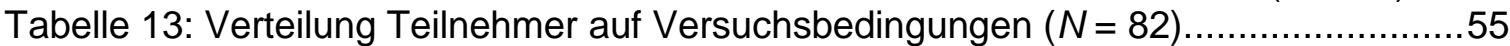

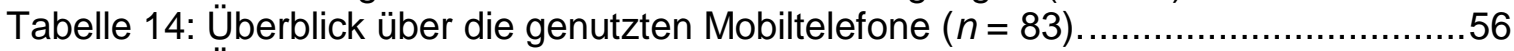

Tabelle 15: Überblick über die Szenarien der Fahrsimulation (Reihenfolge beispielhaft für Anordnung innerhalb der Simulation).

Tabelle 16: Überblick über die verschiedenen Kombinationen für die Fahrten mit Simulationsabläufen und Nebentätigkeiten.

Tabelle 17: Überblick über die sechs Fahrsituationen zur Beanspruchungsmessung. ...63

Tabelle 18: Überblick über Länge der Auswerteintervalle und die ausgewerteten Variablen der einzelnen Szenarien.

Tabelle 19: Anzahl bearbeiteter Zeichen pro Textnachricht, Videodaten.

Tabelle 20: Bedienstrategien beim Lesen - Position des Mobiltelefons im Fahrzeug, Videodaten $(n=33)$.

Tabelle 21: Bedienstrategien beim Schreiben - Position des Handys im Fahrzeug, Videodaten $(n=33)$.

Tabelle 22: Durchschnittliche Dauer und Länge der analysierten geraden

Streckenabschnitte, Videodaten $(n=65)$

Tabelle 23: Anzahl und prozentuale Anteile der Unfälle und Beinaheunfälle mit und ohne Vorinformation bei kritischer Situation Kind, Videodaten.

Tabelle 24: Reaktionszeit nach Sichtbarkeit des Balles, sowie Abstand und Time to Collision zum Kind bei einer Reaktion mit und ohne Vorinformation und die verschiedenen Nebenaufgabenbedingungen, Fahrdaten.

Tabelle 25: Reaktionszeit nach Sichtbarkeit des Baustellenschildes, sowie Abstand und Time to Collision zur Baustelle bei einer Reaktion mit und ohne Vorinformation und die verschiedenen Nebenaufgabenbedingungen, Fahrdaten.

Tabelle 26: Reaktionszeit nach Sichtbarkeit des $30 \mathrm{~km} / \mathrm{h}$ Schildes, sowie Dauer bis zu einer Geschwindigkeitsreduktion um 20\% im Park und im Wohn-und Geschäftsviertel und die verschiedenen Nebenaufgabenbedingungen, Fahrdaten.

Tabelle 27: Einschätzung bei der Beanspruchungsmessung (RSME,

Skala 0 - 150), Anteil der Teilnehmer die Texten würden in \% und die Einschätzung der Erhöhung des Risikos eines Unfalls in \% für die sechs Situationen aus der Simulation, Interviewdaten $(N=82)$. 


\section{Kurzfassung}

\section{Einleitung}

In den letzten Jahren haben Smartphones verstärkt Einzug ins Fahrzeug gehalten. Immer mehr Fahrer bearbeiten während des Fahrens Textnachrichten oder verfassen EMails. Dabei ist bekannt, dass das Lesen und Verfassen von Textnachrichten (im folgenden Texten genannt) das Unfallrisiko erhöhen und allgemein negative Auswirkungen auf die Fahrperformanz haben kann (z.B. stärkere Spurabweichungen, langsamere Reaktionen auf plötzlich auftretende Ereignisse (Caird, Johnston, et al., 2014; Yannis et al., 2016). Bei den meisten dieser Befunde ist allerdings anzumerken, dass es den Teilnehmern in der jeweiligen Untersuchung nicht frei stand selbst zu entscheiden, in welchen Situationen sie sich dem Texten beim Fahren zuwendeten. Vielmehr wurden die Teilnehmer zumeist dazu angehalten, an bestimmten Stellen einer durchfahrenen Strecke (i.d.R. im Rahmen einer Fahrsimulation) eine Textnachricht zu verfassen, unabhängig davon, ob sie dies im realen Verkehr auch getan hätten. Gleichzeitig zeigen aber andere Untersuchungen, dass Fahrer bei der Entscheidung Textnachrichten zu lesen oder zu schreiben durchaus eine Einschätzung der Verkehrssituation vornehmen und die Bearbeitung an deren Anforderungen anpassen. Insofern muss vermutet werden, dass bisherige Befunde zu den Folgen des Lesens und Schreibens von Textnachrichten nur unzureichenden Bezug zum tatsächlichen Geschehen im Straßenverkehr haben. Allerdings ist die Tatsache, dass situative Anpassungen der Nebenaufgabenbearbeitung erfolgen, nicht automatisch Beleg für eine vollständige Kompensation etwaiger negativer Folgen der Fahrerablenkung. So ist unklar, ob die subjektive Bewertung der jeweiligen Fahrsituation, von der die Fahrer ihre Entscheidung für oder gegen das Texten beim Fahren abhängig machen, tatsächlich angemessen ist, und den von der Situation ausgehenden Anforderungen entspricht. Außerdem ist unklar, inwieweit sich neue technische Entwicklungen wie etwa sprachbasierte Systeme zur Ein- und Ausgabe (Vorlesen, Spracheingabe) positiv auf das Fahr- und Reaktionsverhalten auswirken. Zwar gibt es Hinweise, dass zumindest die sprachbasierte Eingabe positive Effekte im Vergleich zur visuell-manuellen Eingabe auf das Fahr- und Reaktionsverhalten haben kann, (He et al., 2014, 2015), jedoch sind diese Befunde mit den gleichen zuvor beschriebenen Problemen behaftet.

Aus dieser Befundlage ergaben sich folgende generelle Forschungsfragen:

- In welchen Kontexten sind Autofahrer zur Bearbeitung von Textnachrichten bereit?

- Welche Auswirkungen haben die visuell-manuelle und die sprachbasierte Bearbeitung von Textnachrichten auf das allgemeine Fahrverhalten (z.B. Geschwindigkeitswahl, Spurhaltung) in Situationen, in denen sich die Fahrer aus freien Stücken für das Ausführen der Nebentätigkeit entscheiden?

- Welche Auswirkungen haben die visuell-manuelle und die sprachbasierte Bearbeitung von Textnachrichten auf das Reaktionsverhalten (z.B. Reaktionszeit, Abstand bei Reaktion) in Situationen, in denen sich die Fahrer aus freien Stücken für das Ausführen der Nebentätigkeit entscheiden, die sich in der Folge aber als sicherheitskritisch erweisen? 


\section{Methodik}

Um die aufgeworfenen Fragen zu beantworten wurden in einem ersten Schritt Kontexte identifiziert, in denen Fahrer bereit sind, Textnachrichten zu lesen oder zu verfassen. Zu diesem Zweck wurden drei Teilstudien durchgeführt:

- eine Literaturanalyse zu Kontexten der Fahrerablenkung,

- eine Re-Analyse der Daten einer naturalistischen Fahrstudie (SHRP2 Naturalistic Driving Study Daten) und

- eine videobasierte Interviewstudie zur Identifikation von Kontexten in denen Fahrer Textnachrichten verfassen.

In der darauffolgenden Fahrsimulatorstudie wurden innerhalb der identifizierten Verkehrskontexte mehr oder weniger kritische Situationen umgesetzt, um die Folgen des Textes beim Fahren zu untersuchen. Die Fahrstrecke wurde dabei so gestaltet, dass sie die Fahrer dazu anregte tatsächlich während der Fahrt Nebentätigkeiten auszuführen. Das heißt, dass die Fahrer allgemein der Ansicht waren, dass das Texten beim Fahren unproblematisch sei. Zusätzlich wurden als potentiell kritische Situationen (1) eine schlecht einsehbare Baustelle und (2) ein Kind, dass auf die Fahrbahn läuft, in die Simulation integriert. Beide Situationen wurden jeweils mit und ohne Vorinformation umgesetzt, die die potentiell kritische Situation ankündigte.

Um der technischen und rechtlichen ${ }^{1}$ Entwicklung Rechnung zu tragen, wurde neben der manuellen Bedienung des Smartphones auch die sprachgesteuerte Ein- und Ausgabe untersucht. Daraus resultierten insgesamt vier Aspekte bzw. Formen des Textens beim Fahren (im Folgenden als Nebentätigkeit oder Nebenaufgabe bezeichnet): (1) Schreiben, (2) Lesen, (3) Spracheingabe und (4) Vorlesen lassen einer Textnachricht. Das Schreiben und Lesen wurde von den Teilnehmern am eigenen Smartphone durchgeführt. Die Spracheingabe und Vorlesefunktion wurden über ein integriertes System in der Mittelkonsole des Fahrzeugs realisiert. Als Vergleich wurde zusätzlich das Fahrverhalten einer Kontrollgruppe erfasst, die keine Nebentätigkeit ausführte. Insgesamt nahmen 82 Fahrer an der Fahrsimulatorstudie teil.

\section{Ergebnisse}

\section{Identifikation von Kontexten}

Die drei Teilstudien zur Identifikation von Kontexten, in denen Fahrer bereit sind, Textnachrichten zu lesen oder zu verfassen, zeigen, dass situative Merkmale von zentraler Bedeutung sind. Entscheidend scheinen übergeordnete Merkmale wie die Komplexität oder Vorhersehbarkeit der Situation zu sein. Bei eher komplexen Situationen bzw. Situationen, die eine erhöhte Aufmerksamkeit des Fahrers benötigen, gaben die Fahrer an weniger häufig zum Mobiltelefon zu greifen. Dies betrifft z.B. kurvige Streckenabschnitte, sowie Fahren bei schlechten Licht- und Sichtbedingungen, hoher Verkehrsdichte und hohen Geschwindigkeiten. In der Interviewstudie zeigte sich, dass

1 Die aktuelle Novelle des §23 StVO („Handyparagraph“) nimmt sprachbasierte Systeme vom Nutzungsverbot während des Fahrens explizit aus (vgl, Quelle: Bundesgesetzblatt Jahrgang 2017 Teil I Nr. 68 (2017). Dreiundfünfzigste Verordnung zur Änderung straßenverkehrsrechtlicher Vorschriften vom 6. Oktober 2017. Bonn, abgerufen am 28.12.2017: https://www.bgbl.de/xaver/bgbl/start.xav\#_bgbl_\%2F\%2F*\%5B\%40attr_id\%3D\%27bgbl117s 3549.pdf\%27\%5D_1514458847080). 
insbesondere die Gegenwart anderer Verkehrsteilnehmer dazu führte, dass das Texten unterlassen wurde. Waren derartige Rahmenbedingungen nicht gegeben, waren die Fahrer durchaus bereit eine Textnachricht zu schreiben oder zu lesen.

\section{Fahrperformanz allgemein}

In der Fahrsimulatoruntersuchung zeigten sich hinsichtlich des generellen Fahrverhaltens ohne kritische Situationen nur vereinzelt statistisch signifikante Effekte der Nebentätigkeitsbearbeitung. So fuhren die Teilnehmer auf einigen Streckenabschnitten, auf denen sie eine Nebenaufgabe ausführten, langsamer als auf Streckenabschnitten ohne Nebenaufgabe. Auch zeigte sich vereinzelt eine geringere Variation der Spurposition, wenn die Teilnehmer eine Nebentätigkeit ausführten.

\section{Fahrperformanz in kritischen Situationen}

In Bezug auf die potentiell sicherheitskritischen Situationen (schlecht einsehbare Baustelle, Kind läuft auf die Straße) zeigten sich uneinheitliche Befunde. So wurden in der schlecht einsehbaren Baustelle keinerlei (Beinahe-) Zusammenstöße registriert, während es bei der Situation mit dem Kind, das auf die Straße lief, in knapp 40\% der Situationen zu einem Unfall oder Beinaheunfall kam. Dabei wurden beim Schreiben von Textnachrichten die meisten Unfälle bzw. Beinaheunfälle registriert, während es zwischen den anderen Nebentätigkeiten (Lesen, Spracheingabe, Vorlesen) kaum Unterschiede gab, auch im Vergleich zum Fahren ohne Nebentätigkeit. In weiteren Indikatoren für das Reaktionsverhalten, wie etwa wann die Reduktion des Gaspedaldrucks oder die Bremspedalbetätigung erfolgte, wurden keine systematischen Unterschiede zwischen den verschiedenen Nebentätigkeiten sowie dem Fahren ohne Nebentätigkeit gefunden. Tendenziell zeigte sich jedoch in einigen dieser Maße, dass die Teilnehmer beim Schreiben einer Nachricht etwas schlechter abschnitten als bei anderen Aufgaben. Hinweisreize auf die potentiell kritische Verkehrssituation (ein Warnschild für die Baustelle bzw. ein auf die Straße rollender Ball beim Kind) hatten die erwarteten positiven Effekte auf das Reaktionsverhalten. Diese Effekte waren unabhängig von der Art der Nebentätigkeit. Auch in Situationen, in denen auf eine veränderte Geschwindigkeitsbegrenzung reagiert werden sollte, wurden keine systematischen Unterschiede zwischen den Nebentätigkeiten sowie dem Fahren ohne Nebenaufgabe beim Zeitpunkt der Geschwindigkeitsreduktion gefunden. Allerdings wurde in Einzelfällen beim Schreiben einer Textnachricht später reagiert.

Insgesamt unterschied sich das Fahrverhalten bei Bearbeitung der meisten Nebentätigkeiten (mit Ausnahme des Schreibens von Textnachrichten) nicht wesentlich vom Fahrverhalten ohne Nebentätigkeit. Insbesondere war dies für die sprachbasierte Ein- und Ausgabe (Vorlesen, Spracheingabe) unerwartet. So wurde angenommen, dass sie schnellere Reaktionen als bei der visuell manuellen Bedienung erlaubt, aber eine langsamere Reaktion als beim Fahren ohne Nebenaufgabe zur Folge hat. Die Ergebnisse zeigen jedoch kein eindeutiges Bild. So unterschieden sich die Fahrleistungen mit sprachbasiertem System nicht systematisch von den Fahrleistungen beim Lesen einer Textnachricht. Gleichzeitig fanden sich aber auch keine klaren Unterschiede zum Fahren ohne Nebenaufgabe.

\section{Diskussion und Schlussfolgerungen}

Aus den ersten drei Untersuchungen ging hervor, dass die Fahrer wenig komplexe und vorhersehbare Situationen für geeigneter für das Texten während des Fahrens hielten als komplexere Situationen. Ebenso verhielt es sich in der durchgeführten Fahrsimulatorstudie. So wurde selbst auf der hier implementierten Strecke, die zum 
Bearbeiten von Nebentätigkeiten einlud, in Kurven häufig darauf verzichtet. Das bedeutet, dass Fahrer bei der Entscheidung Textnachrichten zu lesen oder zu schreiben auf eine subjektive Einschätzung der Verkehrssituation zurückgreifen, und die Bearbeitung an die wahrgenommenen Anforderungen anpassen.

Hinsichtlich der Frage nach den Auswirkungen auf das Reaktionsverhalten in kritischen Situationen zeigten sich sowohl beim manuellen als auch sprachbasierten Texten während des Fahrens kaum Unterschiede zum Fahren ohne Nebentätigkeit. Damit widersprechen die Ergebnisse zumindest in Teilen etablierten Erkenntnissen zu den Folgen von Fahrerablenkung. Eine mögliche Erklärung für diese vermeintliche Diskrepanz ist in der relativ geringen Beanspruchung zu suchen, die von der implementierten Fahrsimulatorstrecke ausging. Diese war so gestaltet, dass sie zum Texten einladen sollte, d.h. möglichst wenig komplex und weitgehend vorhersehbar (bis auf die beiden kritischen Situationen). Dies wurde auch von den Teilnehmern entsprechend so eingeschätzt. Die meisten Verkehrssituationen wurden als wenig bis etwas anstrengend bewertet. Dies könnte dazu geführt haben, dass die Fahrer ohne Nebentätigkeit unterfordert und daher bei der Fahraufgabe unaufmerksam waren. Laut dem Yerkes-Dodson Gesetz (Yerkes and Dodson, 1908) wird die beste Leistung bei mittlerer Beanspruchung erbracht. Sowohl zu geringe als auch zu hohe Beanspruchungen sind mit Leistungseinbußen verbunden. Die sehr geringe Beanspruchung der Strecke könnte sich beim Fahren ohne Nebentätigkeit entsprechend negativ ausgewirkt haben. Vor diesem Hintergrund kann vermutet werden, dass sich die Kontrollgruppe, die ohne Nebenaufgaben fuhr, unterhalb ihres Leistungsmaximums bewegt hat. Dies mag auch erklären, warum Fahrer, im Einklang mit früheren Befunden, Nebentätigkeiten in wenig beanspruchenden Fahrsituationen ohne große Leistungseinbußen ausführen konnten. 


\section{Summary}

\section{Introduction}

In recent years, there has been a considerable increase in smartphone use inside the vehicle. More and more drivers are willing to write text messages or even e-mails while driving. At the same time, it is well established that reading and writing text messages while driving (texting) has the potential to increase crash risk, or, more generally, can negatively impact on driving performance (e.g., increased deviation in lane position, delayed response to unexpected events (Caird, Johnston, et al., 2014; Yannis et al., 2016)). For most of these findings, however, it has to be acknowledged that participants in the respective studies were not free to decide in which driving situation they would text. Instead, they were usually required to start texting on a predefined location on the test track (e.g., during a driving simulator study), regardless of whether they would actually have been willing to do that in real traffic. At the same time, some studies show that drivers decide on whether to text or not based on an assessment of the traffic situation, i.e. they adjust their behaviour according to the requirements of the respective traffic environment. This suggests that the external validity of the studies that find predominantly negative effects of texting in controlled environments is at least limited. At the same time, the fact that drivers adapt their behaviour does not automatically imply a full compensation of potentially negative effects of driver distraction. So far, it is unclear whether the subjective assessment of the traffic situation, which is the basis for the decision to (not) text, is actually appropriate, and indeed reflects the requirements of this situation. Likewise, the potential effects of new technological developments that are intended to reduce the level of distraction and improve driving performance, such as speech based interaction systems (i.e., having the text read aloud, speech based text input) still require proper assessment. While there are a few studies showing that speech based input might have positive effects compared to visual-manual input (He et al., 2014, 2015), they suffer from the same methodological shortcomings as previously described.

Given the described situation, the following research questions arise:

- What are the contexts in which drivers are willing to text?

- What effects do visual-manual and speech based texting (reading and writing) have on general driving behaviour (e.g., speed selection, lane keeping) in situations in which drivers decide voluntarily to attend to the secondary task?

- What effects do visual-manual and speech based texting (reading and writing) have on response behaviour (e.g., response time, distance to hazard when responding) in situations in which drivers decide voluntarily to attend to the secondary task, which, however, then develop into a safety critical event?

\section{Method}

To address the research questions, the first phase of the project was concerned with identifying contexts in which drivers would be willing to read or write text messages. To that end, three separate investigations were conducted:

- An analysis of the literature with regard to the role of context for engagement in driver distraction

- A re-analysis of data from a naturalistic driving study (SHRP2), and

- A video based interview study to identify contexts in which drivers would be willing to text. 
In the second phase of the project, a driving simulator study was conducted, which included driving scenarios in which a variety of more or less safety critical situations were incorporated in the identified contexts. The scenarios were designed in a way that encouraged an engagement in a secondary task while driving, i.e. the drivers would, on average, consider the environment safe enough to text. As potentially safety critical situations, (1) a poorly visible work zone and (2) a child running across the street were integrated in the scenarios. For both situations, a version with and without preceding information (i.e., a traffic sign and a ball rolling across the street) that might facilitate the detection of the critical situation.

To account for technological and regulatory developments ${ }^{2}$, a speech based interaction to read or write text messages was investigated in addition to visual-manual interaction. This resulted in a total of four aspects / forms of texting while driving being investigated in this study (from here on jointly labelled "secondary tasks"): (1) visual-manual text input, (2) reading text message, (3) speech based text input, and (4) listening to text message. Participants used their own smartphones for visual-manual text input and reading. Speech-based interaction and listening were implemented with the help of a seemingly integrated system in the centre console of the vehicle. In addition, the study included a control group, which completed the test drive without engaging in a secondary task. In total, 82 drivers participated in the driving simulator study.

\section{Results}

\section{Identification of contexts}

The three investigations in the role of driving context for the willingness to text showed that indeed, situational characteristics are of vital importance. Of relevance seem to be general aspects such as the complexity or the predictability of the traffic situation. In situations that appear to be complex or seem to require an increased level of attention, drivers reported a reduced rate of mobile phone usage. Examples include curvy road segments, conditions of poor lighting and visibility, high traffic density and high speed. The interview study revealed that especially the presence of other road users would deter drivers from texting. In the absence of such deterring contextual characteristics, drivers showed a general willingness to engage in texting.

\section{General driving performance}

In the driving simulator study, there were only a few isolated significant effects of secondary task engagement on general driving performance. For example, secondary task engagement led to a reduction in speed on some of the analysed road segments. At the same time, secondary task engagement at times appeared to reduce the standard deviation of lane position.

\section{Driving performance in critical situations}

2 The recently revised version of the bill of §23 StVO („Handyparagraph“) explicitly excludes speech based systems from the usage ban of mobile phones while driving (see: Bundesgesetzblatt Jahrgang 2017 Teil I Nr. 68 (2017). Dreiundfünfzigste Verordnung zur Änderung straßenverkehrsrechtlicher Vorschriften vom 6. Oktober 2017. Bonn, abgerufen am 28.12.2017:

https://www.bgbl.de/xaver/bgbl/start.xav\#_bgbl_\%2F\%2F*\%5B\%40attr_id\%3D\%27bgbl117s 3549.pdf\%27\%5D_1514458847080). 
With regard to the potentially safety critical situations (work zone, child crossing), the results were mixed. While there was not a single (near) collision in the work zone scenario, about $40 \%$ of all encounters with the child resulted in a crash or near crash. Writing a text message resulted in the highest proportion of (near) collisions, whereas there was hardly a difference between the other secondary task conditions, including the control group. Other indicators for participants' ability to respond, such as the reduction of throttle pressure or brake pedal use did not show significant differences between the conditions. On a descriptive level, however, performance during visual-manual entry of a text message was somewhat impaired in comparison to the other conditions. Preceding information had the expected positive effect on response behaviour in the potentially critical situations. This effect was independent of secondary task condition. Non-critical situations, in which drivers had to reduce their speed in reaction to a traffic sign indicating a change in the speed limit, did also fail to uncover differences between the different conditions. Only in a few cases, delayed responses were observed during text message entry.

Overall, driver behaviour when engaged in a secondary task (with the exception of the visual-manual entry of a text message) did not visibly differ from driving without secondary task. This was especially unexpected for the speech based interaction, which was assumed to allow for swifter reactions than a visual-manual interaction, but still somewhat slower responses compared to a control group. The results, however, provide no clear evidence in that regard. Performance during speech based interaction was not better than when reading a text message, but also not worse than when driving without secondary task.

\section{Discussion and Conclusions}

The first three investigations showed that drivers seem to prefer situations for texting that are predictable and of limited complexity. Observations from the driving simulator study point in a similar direction, as drivers tended to reduce their engagement in the secondary task when navigating curves, although the simulation as whole was hardly complex or unpredictable. It appears that drivers use a subjective assessment of the traffic situation when deciding to text or not text, and also adjust their engagement dependent on the perceived requirements of the situation.

Regarding the effects of texting on response behaviour in critical situations, neither visual-manual nor speech based interaction did result in clear performance decrements when compared to driving without secondary task. This, to some degree, is contrary to previous findings regarding the effects of driver distraction. One potential explanation might lie in the comparatively low complexity of the implemented simulation environment. This environment was explicitly designed to encourage texting, i.e., it was of low complexity and rather predictable (excluding the two potentially critical situations). Accordingly, when asked, participants rated the demand of the different situations as comparatively low. This might have resulted in an underload of the drivers in the control group (i.e., without secondary task), which, in turn, might have caused inattention. According to the Yerkes-Dodson law (Yerkes and Dodson, 1908), performance is best under conditions of medium workload. Overload, as well as underload, can result in performance decrements. Accordingly, the rather low workload in the control condition can have impacted negatively on performance, which might explain why drivers, in line with previous findings, were able to engage in secondary tasks without clear negative effects in comparatively simple driving scenarios. 


\section{Einführung}

Die Verbreitung von Mobiltelefonen hat weltweit in den letzten zehn Jahren kontinuierlich zugenommen (heise online, 2017). Sie sind zu unserem ständigen Begleiter geworden und damit auch zwangsläufig im Fahrzeug angekommen. Entsprechend hat sich auch die Forschung in den letzten Jahren verstärkt mit der Problematik Mobiltelefonnutzung beim Fahren auseinandergesetzt. Verschiedene Beobachtungsstudien konnten zeigen, dass $3 \%$ bis $4 \%$ der deutschen Autofahrer während des Fahrens mit oder ohne Freisprecheinrichtung telefonierten (DEKRA e.V., 2014; Vollrath et al., 2016). In Deutschland wurden im Jahr 2016 fast 400.000 Verstöße gegen das Verbot der Nutzung eines Mobiltelefons beim Fahren registriert (Kraftfahrtbundesamt, 2017). Diese Zahl ist dabei nicht allein auf das Telefonieren zurückzuführen, welches in der letzten Zeit tatsächlich an Bedeutung verloren zu haben scheint. Stattdessen zeigte eine Befragung, dass insgesamt mehr Fahrer ihr Telefon während des Fahrens zum Schreiben und Lesen von Nachrichten einsetzten als zum Telefonieren (forsa Politik- und Sozialforschung $\mathrm{GmbH}, 2016)$. Dies kann sicher auch daran liegen, dass in den letzten Jahren insbesondere der Anteil Smartphones zugenommen hat (bitkom, 2017). Diese beinhalten ein besonders hohes Ablenkungspotential durch verschiedene Apps wie etwa Messengerdienste. Diesen Trend zum Texten (lesen und schreiben) und Browsen beim Fahren bestätigten auch die Ergebnisse einer Befragung unter deutschen Autofahrern (Kröling \& Gehlert, 2016). Vierzehn Prozent der Befragten gaben an, selten bis sehr oft eine SMS oder E-Mail beim Fahren zu schreiben, während sogar 27\% entsprechende Nachrichten lesen. In einer Beobachtungsstudie von Vollrath und Kollegen (2016) in drei deutschen Städten wurde gezeigt, dass ca. 5\% der Fahrer während des Fahrens Textnachrichten lasen oder schrieben. Auch internationale Studien zeigen, dass dieses Verhalten häufig vorkommt. In Befragungen berichteten zwischen knapp 20\% und $40 \%$ der Fahrer während des Fahrens Textnachrichten zu lesen oder zu schreiben (Vollrath et al., 2014; Kubitzki \& Fastenmeier, 2016; Prat et al., 2017). In einer US-amerikanischen Untersuchung berichteten sogar über $90 \%$ der Befragten im letzten Jahr während des Fahrens getextet zu haben, wobei es keine Unterschiede zwischen Männern und Frauen gab (Struckman-Johnson et al., 2015). Durch die neuen und stetig wachsenden Kommunikationsmöglichkeiten und auch -erfordernisse, die durch die Nutzung von Applikationen wie Facebook, WhatsApp usw. entstehen, ist zu erwarten, dass diese Zahlen weiter ansteigen.

\subsection{Auswirkungen des Lesens und Schreibens von Textnach- richten auf die Verkehrssicherheit}

Um die Gefährdung der Verkehrssicherheit durch Texting einschätzen zu können, erfolgte eine wissenschaftliche Betrachtung der Auswirkungen des Lesens und Schreibens von Textnachrichten auf das Fahrverhalten. In einigen Untersuchungen wurde dabei zwischen dem Lesen und Schreiben von Textnachrichten unterschieden, während in anderen Arbeiten das Texten als ganzheitliche Aufgabe (Lesen und Schreiben gemeinsam) betrachtet wurde (Caird, Johnston, et al., 2014). Aussagekräftiger ist in diesem Zusammenhang die Beobachtung der Konsequenzen des Textens in einem realistischen Fahrumfeld, in dem speziell Aspekte wie Unfallhäufigkeiten deutlich besser zu untersuchen sind als etwa in einem Fahrsimulator (Dingus et al., 2016; Simmons, Hicks \& Caird, 2016). Dabei konnte gezeigt werden, dass Fahrer, die durch das Lesen oder Schreiben einer Textnachricht abgelenkt waren, ein deutlich höheres Unfallrisiko aufwiesen als Fahrer ohne Nebentätigkeit, ebenso wie auch im Vergleich zu Fahrern, die anderen Nebentätigkeiten wie etwa Telefonieren oder 
Essen nachgingen (Caird, Johnston, et al. 2014; Dingus et al. 2016; Simmons et al. 2016).

Um jedoch die Umstände und Folgen im Detail untersuchen zu können, wurde der überwiegende Teil der Studien im Simulator umgesetzt. Dort können unter kontrollierten Bedingungen und in einer sicheren Umgebung die Auswirkungen des Textens klar von anderen Einflüssen abgegrenzt untersucht werden (z.B. He et al., 2014; Yannis et al., 2014; Schömig et al., 2015). In bisherigen Untersuchungen im Simulator wurden die Fahrer zumeist dazu angehalten, auf einem bestimmten Streckenabschnitt eine Textnachricht zu schreiben oder zu lesen (McKeever et al., 2013; He et al., 2014). Dabei wurden die Auswirkungen auf verschiedene Variablen untersucht, die mit Unfällen oder auch deren Vermeidung im Zusammenhang stehen, wie die Reaktion auf plötzlich auftretende Ereignisse (z.B.Bremsverhalten), Spurhaltung oder Kompensationsverhalten (z.B. Geschwindigkeitswahl, Abstand zum vorausfahrenden Fahrzeug).

In Bezug auf die Reaktionsfähigkeit der Fahrer zeigten sich deutlich negative Effekte bei plötzlichem Bremsen eines vorausfahrenden Fahrzeugs (Drews et al., 2009; Yannis et al., 2016). Die Reaktionszeit war verlängert, wenn die Fahrer mit dem Texten befasst waren. Dieser Effekt konnte ebenfalls gefunden werden, wenn andere plötzlich auftretende Ereignisse bemerkt werden sollten (Libby, Chaparro \& He, 2013; Yannis et al., 2014). In einer Studie von Choudhary und Velaga (2017) wurde zusätzlich zwischen dem Schreiben von kurzen ( $<10$ Zeichen) und längeren Textnachrichten ( $>10$ Zeichen) unterschieden. Gleichzeitig sollten die Fahrer dabei auf einen querenden Fußgänger oder querendes Fahrzeug reagieren. Die Autoren fanden längere Reaktionszeiten, wenn die Fahrer Textnachrichten verfassten, wobei insbesondere die längeren Nachrichten zu Beeinträchtigungen führten. Schömig und Kollegen (2015) allerdings konnten zeigen, dass, wenn die Fahrer frei wählen konnten, wann sie eine Eingabe tätigten, eine Bremsung als Reaktion auf einen plötzlich auftauchenden Fußgänger ähnlich schnell erfolgte wie wenn keine Nebenaufgabe bearbeitet wurde. Bei Personen, die zu einer Bearbeitung angehalten wurden (d.h. nicht frei entscheiden konnten), wurde eine verspätete Reaktion festgestellt. Auch zeigte die Studie, dass die Fahrer vor allem in kritischen Situationen die Beschäftigung mit der Nebenaufgabe deutlich verringerten. Die Autoren schlossen daraus, dass Fahrer durchaus in der Lage seien Verkehrssituationen adäquat zu antizipieren und die Bearbeitung der Nebenaufgabe entsprechend anzupassen.

Auch für die Spurhaltung wurden zumeist negative Effekte des Schreibens und Lesens von Textnachrichten berichtet (Caird, Johnston, et al. 2014). So verließen die Fahrer signifikant häufiger die Spur, wenn sie durch Texten abgelenkt waren als wenn sie nur das Fahrzeug steuerten (Hosking, Young \& Regan, 2009; Basacik, Reed \& Robbins, 2011). Auch fanden sich wiederholt deutlich größere Abweichungen von der Spurposition beim Texten im Vergleich zum Fahren ohne Nebentätigkeit (Leung et al., 2012; RudinBrown et al., 2013).

Um die beschriebenen negativen Effekte des Lesens und Schreibens von Textnachrichten auszugleichen, reagieren Fahrer oft mit Kompensationsverhalten. So wurde wiederholt berichtet, dass Fahrer die Geschwindigkeit reduzierten wenn sie eine Textnachricht lasen und schrieben (Libby, Chaparro \& He, 2013; Rudin-Brown et al., 2013; Yannis et al., 2014, 2016). Auch Vergrößerungen des Abstands zum Vorausfahrzeug wurden gefunden (Drews et al., 2009; Yannis et al., 2016). Gleichzeitig kam es auch zu mehr Schwankungen im Abstand zum Vorausfahrzeug (Drews et al., 2009), was wiederum auf eine Beeinträchtigung durch die Nebenaufgabenbearbeitung schließen lässt. 


\subsection{Auswirkungen des sprachgesteuerten Abhörens und Ver- fassens von Textnachrichten auf die Verkehrssicherheit}

Nicht zuletzt in Reaktion auf die potentiellen Gefahren der visuell-manuellen Interaktion mit einem Mobiltelefon sind in den Neufahrzeugen der vergangenen Jahre zunehmend Freisprecheinrichtungen, und seit neustem auch vermehrt sprachgesteuerte Systeme für die Eingabe und das Abhören von Textnachrichten zu finden. Diese Systeme sollen den Fahrer dadurch unterstützen, dass die Hände am Steuer verbleiben können, und auch die Notwendigkeit visueller Zuwendung reduziert wird. Es besteht Einigkeit darüber, dass ein zentrales Problem bei einer Reihe von im Fahrzeug zu beobachtenden Nebentätigkeiten darin besteht, dass die Fahrer zur Bearbeitung wiederholt den Blick von der Straße abwenden müssen. Diese Blickabwendung scheinen in Zusammenhang mit einem höheren Unfallrisiko zu stehen (Klauer et al., 2006; Simons-Morton et al., 2014). In diesem Sinn sollen neue Systeme wie etwa Spracheingabe oder Vorlesefunktion im Fahrzeug den Fahrer entlasten und durch verminderte Blickabwendung für eine höhere Sicherheit sorgen. Dem wird auch in der Novellierung des entsprechenden Paragraphen der StVO zur Nutzung des Mobiltelefons am Steuer (§ 23) Rechnung getragen. Dieser erlaubt in der neuen Fassung unter Punkt 2a die Nutzung einer Sprachsteuerung und Vorlesefunktion ohne manuelle Bedienung des Geräts (Bundesministerium für Verkehr und digitale Infrastruktur \& Bundesministerium für Umwelt, Naturschutz, Bau und Reaktorsicherheit, 2017).

Zum Telefonieren mit Freisprechanalage gibt es bereits eine vergleichsweise lange Forschungstradition (Alm \& Nilsson, 1994; Törnros \& Bolling, 2006; Lipovac et al., 2017), während zur sprachbasierten Eingabe oder dem Abhören von Textnachrichten noch relativ wenige Arbeiten existieren. In einer Studie von He und Kollegen (2014) mussten die Teilnehmer entweder eine Nachricht manuell oder sprachbasiert eingeben. Die Ergebnisse deuten darauf hin, dass die sprachbasierte Eingabe weniger schwerwiegende Konsequenzen für die Fahrleistung hat wie die manuelle Eingabe, sich in ihren Auswirkungen aber dennoch von einer Fahrt ohne Nebentätigkeit unterscheidet.

So fanden die Autoren eine größere Spurabweichung und stärkere Geschwindigkeitsschwankungen als wenn die Fahrer keiner Nebentätigkeit nachgingen. Gleichzeitig wurde aber bei der Spracheingabe ähnlich schnell auf das Abbremsen des Vorausfahrzeugs reagiert wie beim Fahren ohne Nebentätigkeit. Andere Studien fanden allerdings sehr wohl längere Reaktionszeiten bei Spracheingabe im Vergleich zum reinen Fahren fanden (Yager, 2013b; He et al., 2015). Außerdem fanden sich negative Effekte bei der Spurhaltung und Geschwindigkeit (He et al., 2015; Paridon, Hofmann \& Schreiber, 2015). Allerdings konnten He, Choi, McCarley, Chaparro und Wang (2015) zeigen, dass ein sicherheitsförderliches Kompensationsverhalten stattfand, in dem bei der Eingabe von Textnachrichten sowohl manuell als auch sprachbasiert größere Abstände zum Vorausfahrzeug gehalten wurden als wenn die Teilnehmer keiner Nebentätigkeit nachgingen. Es bleibt festzuhalten, dass die wenigen bislang veröffentlichten Arbeiten ein uneinheitliches Bild der möglichen Effekten von sprachbasierten Eingabesystemen auf die Verkehrssicherheit zeichnen. Auch lag der Schwerpunkt bislang auf Betrachtungen des Verfassens (manuell oder sprachbasiert) von Nachrichten, während zur Vorlesefunktion nach jetzigem Stand anscheinend noch keine Befunde vorliegen.

\subsection{Projektbeschreibung}

Die meisten Untersuchungen deuten auf negative Auswirkungen des Lesens und Schreibens von Textnachrichten beim Fahren hin. Einschränkend ist jedoch anzumerken, dass es den Teilnehmern in den bisherigen Studien zumeist nicht freistand, 
ob bzw. wann sie neben dem Fahren eine Nebenaufgabe ausführten, egal wie komplex oder schwierig die Fahrsituation war. Daher gibt es Zweifel an der Übertragbarkeit dieser Befunde auf die tatsächliche Verkehrsumgebung. Es ist fraglich, ob mit einem solchen Vorgehen realistisches Nutzungs-, Bedien- und Fahrverhalten beobachtet werden kann, wie etwa die Befunde zu Verhaltensanpassungen bei der Nebenaufgabenbearbeitung von Schömig und Kollegen (2015) nahelegen. Inwieweit diese Anpassungen allerdings ausreichend sind um das durch Nebenaufgabenbearbeitung erhöhte Unfallrisiko zu reduzieren ist unklar. So sind die zu beobachtenden Kompensationsstrategien (Reduktion der Geschwindigkeit, Vergrößerung von Sicherheitsabständen) in erster Linie darauf ausgelegt, dem Fahrer mehr Zeit bei eventuellen Reaktionen zu verschaffen, können aber nicht verhindern, dass potentielle Hinweisreize oder gar ganze kritische Situationen übersehen bzw. nicht als solche identifiziert werden. Ebenso ist unklar, ob die subjektive Bewertung der jeweiligen Fahrsituation, von der die Fahrer ihre Entscheidung für oder gegen die Nebenaufgabenbearbeitung abhängig machen, tatsächlich angemessen ist, und den von der Situation ausgehenden Anforderungen entspricht. Um derartige Fragen unter ungefährlichen und kontrollierten Bedingungen untersuchen zu können, und gleichzeitig dem Fahrer die Anpassung des Textens an die jeweilige Situation zu ermöglichen, erschien eine fahrsimulatorbasierte Untersuchung sinnvoll, in der der Fahrer frei über die Bearbeitung einer Nebentätigkeit entscheiden kann, in der aber gleichzeitig die Strecke so gestaltet ist, dass sie den Fahrer eher dazu anregt, tatsächlich einer Nebentätigkeit nachzugehen.

Im Vorfeld der Fahrsimulatorstudie war es zunächst nötig Kontexte zu identifizieren, in denen Fahrer bereit sind Textnachrichten zu lesen oder zu schreiben. Zu diesem Zweck erfolgten eine Literaturanalyse (Kapitel 2), eine Re-Analyse der Daten einer naturalistischen Fahrstudie (SHRP2, Kapitel 3) und eine Interviewstudie mit Autofahrern, von denen eine grundsätzliche Bereitschaft zum Texten während des Fahrens bekannt war (Kapitel 4). In der nachfolgenden Fahrsimulatorstudie (Kapitel 5) wurden innerhalb dieser identifizierten Kontexte konkrete, mehr oder weniger kritische Situationen umgesetzt, um die Folgen der Nebenaufgabenbearbeitung speziell in den Fällen untersuchen zu können, in denen Fahrer gemeinhin der Ansicht sind, dass diese Nebenaufgabenbearbeitung unproblematisch ist. In Anbetracht der vermeintlichen Vorteile einer sprachgesteuerten gegenüber einer visuell-manuellen Interaktion erfolgte im Rahmen der Untersuchung eine detaillierte Betrachtung beider Bedienmöglichkeiten.

Es ergaben sich folgende generelle Forschungsfragen:

- In welchen Kontexten sind Autofahrer zur Bearbeitung von Textnachrichten bereit?

- Welche Auswirkungen haben die visuell-manuelle und die sprachbasierte Bearbeitung von Textnachrichten auf das allgemeine Fahrverhalten (z.B. Geschwindigkeitswahl, Spurhaltung) in Situationen, in denen sich die Fahrer aus freien Stücken für die Bearbeitung der Nebenaufgabe entscheiden?

- Welche Auswirkungen haben die visuell-manuelle und die sprachbasierte Bearbeitung von Textnachrichten auf das Reaktionsverhalten (z.B. Reaktionszeit, Abstand bei Reaktion) in Situationen, in denen sich die Fahrer aus freien Stücken für die Bearbeitung der Nebenaufgabe entscheiden, die sich in der Folge aber als sicherheitskritisch erweisen? 


\section{Literaturanalyse zu Kontexten der Fahrerablenkung}

Um Kontexte zu identifizieren, in denen die Fahrer bereit sind Textnachrichten zu lesen und/oder zu schreiben, wurde zunächst eine Literaturrecherche durchgeführt. Hierbei wurden nicht nur Studien berücksichtigt, bei denen die Teilnehmer eine Textnachricht verfassten oder lasen, sondern auch Untersuchungen, bei denen eine andere Art von visuell-manueller Aufgabe am Mobiltelefon ausgeübt wurde, z.B. Eingabe einer Telefonnummer. Zusätzlich wurden Studien einbezogen, bei denen vergleichbaren artifiziellen Nebentätigkeiten nachgegangen wurde. So sollten die Probanden in einigen Untersuchungen Zahlen auf einem Display vorlesen, was weitestgehend dem Lesen einer Textnachricht entspricht. In anderen Arbeiten war das Abtippen von Zahlen von einem Display gefordert, was dem Lesen und Schreiben einer Textnachricht sehr nahekommt. Des Weiteren wurden in den Untersuchungen verschiedenste methodische Ansätzen verwendet. So fanden sich naturalistische Fahrstudien (Tivesten \& Dozza, 2014, 2015; Xiong et al., 2015), stationäre Beobachtungen (Huth, Sanchez \& Brusque, 2014; Kidd et al., 2016; Vollrath et al., 2016), Simulatorstudien (Rauch, Gradenegger \& Krüger, 2008, 2009; Schömig \& Metz, 2013; Schömig et al., 2015) und Befragungen (Young \& Lenné, 2010).

Ziel der Literaturanalyse war es Untersuchungsergebnisse zusammenzutragen, die Aussagen darüber erlauben, welche Kontextvariablen mit einer erhöhten oder auch verminderten Bereitschaft zum Texten bzw. zur Bearbeitung ähnlicher Aufgaben einhergehen. Ebenso wurden dabei auch einige Variablen gefunden, bei denen kein eindeutiger Zusammenhang auszumachen war, d.h. bei denen sich, teilweise entgegen der ursprünglichen Annahmen der Autoren, keine Effekte auf die Bereitschaft zum Texten zeigten. Tabelle 1 gibt einen Überblick über die Kontextfaktoren, die mit der Bereitschaft zum Lesen und Schreiben von Textnachrichten oder ähnlichen Nebentätigkeiten im Zusammenhang stehen. Es ist erkennbar, dass die Mehrheit der identifizierten Faktoren mit einer verringerten Bereitschaft zur Nebenaufgabenbearbeitung einherging. So wurden mehrere Kontextmerkmale identifiziert, bei denen davon ausgegangen werden kann, dass sie eine erhöhte Aufmerksamkeit bei der Fahrzeugkontrolle erfordern (hohe Geschwindigkeit, Überholmanöver). Bei einigen weiteren identifizierten Faktoren ist eine stärkere Aufmerksamkeitszuwendung zur Umgebung zu vermuten, entweder weil die Komplexität der Situation allgemein erhöht ist (dichter Verkehr, entgegenkommendes Fahrzeug, schlechtes Wetter, schlechte Lichtverhältnisse, kurvige Strecke), oder aber weil besonders auf andere Personengruppen Rücksicht genommen werden muss (Schule in der Nähe, Fußgängerüberwege). Auch die Präsenz von Mitfahrern scheint mit einer reduzierten Bereitschaft zum Texten einherzugehen. Demgegenüber stehen Faktoren wie niedrige Geschwindigkeiten bzw. Stillstand oder kein Gegenverkehr, für die eine erhöhte Bereitschaft zu Texten berichtet wurde. Auch im Stadtverkehr wird scheinbar verstärkt getextet, wobei vermutet werden kann, dass dies u.a. durch die erhöhte Präsenz von Lichtsignalanlagen (und damit der Notwendigkeit, dort zu stoppen) in dieser Umgebung zu erklären ist. Für die Präsenz vorausfahrender Fahrzeuge, Tageslicht (d.h. gute Beleuchtungs-/Sichtverhältnisse) und das gerade Durchfahren einer Kreuzung wurden keine eindeutigen Ergebnisse berichtet. Zusammenfassend lässt sich aber feststellen, dass eine hohe Schwierigkeit oder Komplexität einer Situation dazu beiträgt, dass weniger Nebentätigkeiten, während in einfachen Fahrsituationen in wenig komplexen Umgebungen mehr Nebentätigkeiten ausgeübt werden. 
Tabelle 1: Überblick über Kontexte oder Umgebungsbedingungen und der Bereitschaft Nebentätigkeiten nachzugehen.

\begin{tabular}{|c|c|c|}
\hline Kontextvariable & Quelle & Nebentätigkeit (It. Quelle) \\
\hline \multicolumn{3}{|c|}{$\begin{array}{c}\text { Kontextvariablen, bei denen eine erhöhte Bereitschaft Nebentätigkeiten nachzugehen } \\
\text { gefunden wurde }\end{array}$} \\
\hline \begin{tabular}{l|} 
Niedrige \\
Geschwindigkeit bzw. \\
Stillstand
\end{tabular} & $\begin{array}{l}\text { (1) Tivesten \& Dozza (2015) } \\
\text { (2) Tivesten \& Dozza (2014) } \\
\text { (3) Xiong et al. (2014) } \\
\text { (4) Rauch et al. (2008) } \\
\text { (5) Vollrath et al. (2016) } \\
\text { (6) Huth et al. (2014) } \\
\text { (7) Kidd et al. (2016) }\end{array}$ & $\begin{array}{l}(1,2,3,5,6,7) \text { Visuell-manuelle } \\
\text { (VM) Telefonaufgabe (Texting, } \\
\text { Lesen, Wählen); } \\
\text { (4) Zahlen vom Display vorlesen }\end{array}$ \\
\hline Innerhalb Stadt & $\begin{array}{l}\text { (1) Rauch et al. (2008) } \\
\text { (2) Rauch et al. (2009) }\end{array}$ & $\begin{array}{l}(1,2) \text { Zahlen vom Display } \\
\text { vorlesen }\end{array}$ \\
\hline Kein Gegenverkehr & Schömig et al. (2015) & Zahlen abtippen \\
\hline $\begin{array}{l}\text { Geradeausfahrten } \\
\text { (außerorts) }\end{array}$ & $\begin{array}{l}\text { (1) Rauch et al. (2008) } \\
\text { (2) Schömig et al. (2015) }\end{array}$ & $\begin{array}{l}\text { (1) Zahlen vom Display vorlesen } \\
\text { (2) Zahlen abtippen }\end{array}$ \\
\hline $\begin{array}{l}\text { Lichtverhältnisse: } \\
\text { Dämmerung, Nacht }\end{array}$ & $\begin{array}{l}\text { (1) Tivesten \& Dozza (2015) } \\
\text { (2) Kidd et al. (2016) }\end{array}$ & $(1,2)$ VM-Telefonaufgabe \\
\hline Autobahn, Landstraße & Tivesten \& Dozza (2015) & VM-Telefonaufgabe \\
\hline \multicolumn{3}{|c|}{$\begin{array}{c}\text { Kontextvariablen ohne eindeutigen Einfluss auf die Bereitschaft Nebentätigkeiten } \\
\text { nachzugehen }\end{array}$} \\
\hline $\begin{array}{l}\text { Gerade durchfahren } \\
\text { einer Kreuzung }\end{array}$ & Tivesten \& Dozza (2015) & VM-Telefonaufgabe \\
\hline $\begin{array}{l}\text { Vorausfahrendes } \\
\text { Fahrzeug }\end{array}$ & $\begin{array}{l}\text { (1) Tivesten \& Dozza (2015) } \\
\text { (2) Tivesten \& Dozza (2014) }\end{array}$ & $(1,2)$ VM-Telefonaufgabe \\
\hline Tageslicht & Tivesten \& Dozza (2015) & VM-Telefonaufgabe \\
\hline \multicolumn{3}{|c|}{$\begin{array}{c}\text { Kontextvariablen, bei denen eine verringerte Bereitschaft Nebentätigkeiten nachzugehen } \\
\text { gefunden wurde }\end{array}$} \\
\hline $\begin{array}{l}\text { Hohe } \\
\text { Geschwindigkeit }\end{array}$ & $\begin{array}{l}\text { (1) Tivesten \& Dozza (2015); } \\
\text { (2) Tivesten \& Dozza (2014); } \\
\text { (3) Xiong et al. (2014) }\end{array}$ & $(1,2,3)$ VM-Telefonaufgabe; \\
\hline $\begin{array}{l}\text { Spurwechsel/ } \\
\text { Überholen }\end{array}$ & $\begin{array}{l}\text { (1) Tivesten \& Dozza (2015); } \\
\text { (2) Schömig et al. (2015) }\end{array}$ & $\begin{array}{l}\text { (1) VM-Telefonaufgabe; } \\
\text { (2) Zahlen abtippen }\end{array}$ \\
\hline Dichter Verkehr & Young \& Lenné (2010); & $\begin{array}{l}\text { mehrere Zweitaufgaben u.a. } \\
\text { Texten }^{3}\end{array}$ \\
\hline
\end{tabular}

${ }^{3}$ Obwohl in der Arbeit von Young und Lenné (2010) das Texten als separate Verhaltensweise erfasst wurde, wurde in den Analysen zur Rolle verschiedener Kontextvariablen nicht zwischen dem Texten und anderen Nebenaufgaben unterschieden. Entsprechende Aussagen über alle Nebenaufgaben (inklusive Texten) hinweg. 


\begin{tabular}{|c|c|c|}
\hline Kontextvariable & Quelle & Nebentätigkeit (It. Quelle) \\
\hline $\begin{array}{l}\text { Entgegenkommendes } \\
\text { Fahrzeug } \\
\text { (ländliche Straße) }\end{array}$ & Tivesten \& Dozza (2014) & VM-Telefonaufgabe \\
\hline Schlechtes Wetter & Young \& Lenné (2010) & $\begin{array}{l}\text { mehrere Zweitaufgaben u.a. } \\
\text { Texten }\end{array}$ \\
\hline $\begin{array}{l}\text { Lichtverhältnisse: } \\
\text { Nacht }\end{array}$ & Young \& Lenné (2010) & $\begin{array}{l}\text { mehrere Zweitaufgaben u.a. } \\
\text { Texten }\end{array}$ \\
\hline $\begin{array}{l}\text { Enge Kurven, } \\
\text { Abbiegen, } \\
\text { Kreisverkehr, } \\
\text { kurze Entfernung } \\
\text { zur Kurve }\end{array}$ & $\begin{array}{l}\text { (1) Tivesten \& Dozza (2015) } \\
\text { (2) Tivesten \& Dozza (2014) } \\
\text { (3) Xiong et al. (2014) } \\
\text { (4) Young \& Lenné (2010) } \\
\text { (5) Schömig et al. (2013) } \\
\text { (6) Rauch et al. (2008) } \\
\text { (7) Basacik et al. (2011) } \\
\text { (8) Schömig et al. (2011) } \\
\text { (9) Kidd et al. (2016) }\end{array}$ & $\begin{array}{l}(1,2,3,7,9) \text { VM-Telefonaufgabe; } \\
(4) \text { Mobiltelefonnutzung; } \\
(5,6,8) \text { Zahlen vom Display } \\
\text { vorlesen }\end{array}$ \\
\hline Schule in der Nähe & Young \& Lenné (2010) & $\begin{array}{l}\text { mehrere Zweitaufgaben u.a. } \\
\text { Texten }\end{array}$ \\
\hline Fußgängerüberwege & Rauch et al. (2008) & Zahlen vom Display vorlesen \\
\hline $\begin{array}{l}\text { Fußgänger } \\
\text { überqueren Straße }\end{array}$ & Schömig et al. (2015) & Zahlen abtippen \\
\hline Mitfahrer & $\begin{array}{l}\text { (1) Tivesten \& Dozza (2015) } \\
\text { (2) Vollrath et al., (2016) }\end{array}$ & $\begin{array}{l}\text { (1) VM-Telefonaufgabe } \\
\text { (2) mehrere Zweitaufgaben u.a. } \\
\text { Texten }\end{array}$ \\
\hline
\end{tabular}

Anmerkung: $\mathrm{VM}=$ visuell manuell 


\section{Re-Analyse der SHRP2 Naturalistic Driving Study Daten}

Eine übergeordnete Zielstellung dieser Untersuchung bestand darin Kontexte zu identifizieren in denen Fahrer verstärkt dazu neigen Textnachrichten (z.B. SMS, WhatsApp-Nachrichten, E-Mails, Facebook-Nachrichten) während des Fahrens zu schreiben. Hierfür wurden die Daten einer naturalistischen Fahrstudie herangezogen, bei der die Nebentätigkeiten der Fahrer sowie diverse Kontextvariablen erfasst wurden.

\subsection{Beschreibung der SHRP2 NDS}

Im Rahmen des Second Strategic Highway Research Program wurde in den USA von 2006 bis 2015 eine großangelegte naturalistische Fahrstudie (Naturalistic Driving Study) durchgeführt (im folgenden SHRP2 NDS genannt). Ziel der SHRP2 NDS war die Untersuchung von Fahrverhalten und Fahrperformanz, mit Schwerpunkt Verkehrssicherheit. Der Fokus lag darauf zu verstehen wie Fahrer mit ihrem Fahrzeug, der Verkehrsumgebung, Verkehrskontrollsystemen und anderen Umwelteinflüssen interagieren und sich daran anpassen (Campbell, 2012). Es wurden insgesamt mehr als 3.300 Fahrzeuge in 6 verschiedenen Regionen der USA (Bloomington, Indiana; Central Pennsylvania; Tampa Bay, Florida; Buffalo, New York; Durham, North Carolina; Seattle, Washington) mit einem Datenaufzeichnungssystem ausgestattet (Dingus et al., 2015). Damit ist die SHRP2 NDS ist die größte naturalistische Fahrstudie, die bisher durchgeführt wurde. Der überwiegende Teil der Fahrzeuge waren Pkw sowie einige Vans und SUV's. Lkw und motorisierte Zweiräder wurden explizit nicht berücksichtigt. Die Fahrzeuge der Teilnehmer waren mit einer Vielzahl unterschiedlicher Instrumente zur Datenaufzeichnung ausgestattet, darunter u.a. Videokameras nach vorn, nach hinten, auf das Gesicht des Fahrers und das Armaturenbrett. Die Aufnahmen dieser verschiedenen Kameras konnten zur Identifikation und Analyse der Nebentätigkeiten herangezogen werden. Ferner war weitere Sensortechnik, z.B. zur Erfassung der Geschwindigkeit, Positionsbestimmung, Bremspedalbetätigung etc., verbaut. Einen genauen Überblick über die Ausstattung gibt Dingus et al. (2015). Insgesamt wurden mehr als 35 Millionen Meilen (56 Mio. km) von kontinuierlichen Fahrdaten und 2 Petabyte (PB) von Video-, Kinematik- und Audiodaten aufgezeichnet (Dingus et al., 2016). Eine der Analysen im Rahmen der SHRP2 NDS beschäftigte sich mit Fahrerunaufmerksamkeit und Unfallrisiko (Campbell, 2012). Die NDS Daten bieten die Möglichkeit zu untersuchen wie sich Fahrerablenkung bzw. -unaufmerksamkeit in Kombination mit Bedingungen der Fahrumgebung auf das Unfallrisiko auswirken. Aus diesem Grund wurden auch die Nebentätigkeiten der Fahrer sowie diverse Kontextvariablen der Umgebung miterfasst. Diese NDS Daten sind insbesondere dafür geeignet die Frage zu klären, in welchen Kontexten Fahrer besonders häufig Textnachrichten während des Fahrens schreiben oder Tätigkeiten im Internet (z.B. Mails schreiben, Webseiten besuchen) am Mobiltelefon ausführen.

\subsection{Methode der SHRP2 Studie}

\subsubsection{Stichprobe}

\section{Ausgangsstichprobe in SHRP2}

Über einen Zeitraum von insgesamt drei Jahren wurden mehr als 3.500 Teilnehmer während ihrer Fahrten beobachtet (Dingus et al., 2016). Die meisten Probanden wurden dabei über einen Zeitraum von ein bis zwei Jahre begleitet. Voraussetzungen für die Teilnahme an der Studie waren das Vorhandensein eines aktuellen Führerscheins, der 
Besitz eines Fahrzeuges bzw. die Erlaubnis zur Nutzung seitens des Besitzers, sowie ein Wohnsitz innerhalb des Studienbereiches. Ferner sollten die Teilnehmer mindestens drei Mal pro Woche fahren, und mindestens 3.000 Meilen (ca. 4.800 km) im Jahr zurücklegen (Dingus et al., 2015).

Verwendete Stichprobe für die Re-Analyse der Daten

Die für diese Auswertung verwendete Stichprobe umfasst 2.884 Fahrer. Die Altersspanne betrug dabei 16 bis 99 Jahre $^{4}$. Es war ein höherer Anteil junger Fahrer 20 bis 24 Jahre (20\%) beteiligt. Einen Überblick über Alters- und Geschlechtsverteilung gibt Abbildung 1.

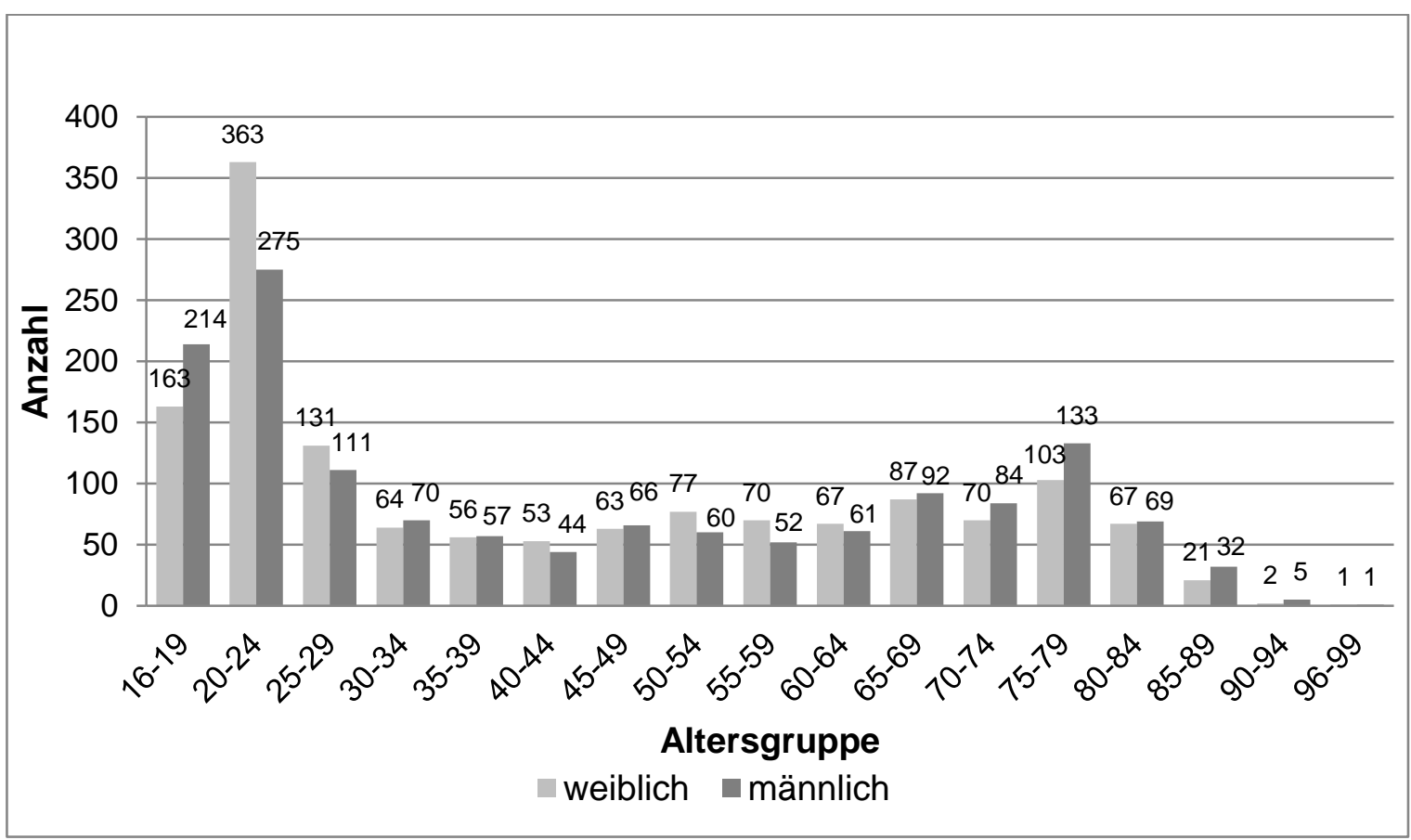

Abbildung 1: verwendete Stichprobe des SHRP2 Datensatzes nach Altersgruppen.

\subsubsection{Kodierung der SHRP2 Daten}

\section{Bestehende Kodierung der SHRP2 NDS}

Im Rahmen der SHRP2 NDS wurde eine Vielzahl unterschiedlicher Fahrzeugvariablen, Fahrervariablen, Fahrtvariablen sowie Ereignisvariablen erhoben. Da das primäre Ziel der SHRP2 NDS auf Fragen der Verkehrssicherheit lag, wurde im Datensatz zunächst nach Unfällen und vergleichbaren Konfliktsituationen gesucht, um diese umfassend beschreiben zu können. Für diese Situationen wurde eine umfangreiche Anleitung zur Kodierung der Videos erstellt (Virginia Tech Transportation Institute, 2015). Anhand dieser Anleitung wurden dann die Variablen (darunter Nebentätigkeiten und Kontextvariablen) aus den Videos der Fahrten von den Mitarbeitern des Virginia Tech Transportation Institute kodiert und deren Häufigkeit bestimmt. Um die Berechnung von Odds-Ratios und damit die Identifikation von potentiellen Risikofaktoren zu ermöglichen,

4 Dabei ist anzumerken, dass im Datensatz das Alter der Teilnehmer lediglich in den in Abbildung 1 dargestellten Kategorien festgehalten ist. Das genaue Alter der Teilnehmer ist demnach nicht bekannt. 
wurden zudem sogenannte Baselineabschnitte identifiziert, in denen entsprechend die gleichen Variablen kodiert wurden wie in den Konfliktsituationen. Diese kodierten Datensätze sind öffentlich zugänglich über die Website https://insight.shrp2nds.us/. Die Daten sind über eine Datenbank abrufbar und durch Filter kann man sich eine Auswahl an relevanten Variablen anzeigen lassen, sowie die Variablen verschiedenartig kombinieren. Über die Website besteht allerdings kein Zugang zu den Sensordaten (z.B. Geschwindigkeit, Bremspedalbetätigung etc.) der Fahrten, so dass diese Informationen für die Auswertung nicht zur Verfügung standen. Ebenso sind keine Videodaten der Fahrten verfügbar, so dass keine eigenständigen Kodierungen vorgenommen werden konnten.

\section{Verwendete kodierte Variablen für die Identifikation der Kontexte}

Für die vorliegende Analyse der Daten wurde aus den bestehenden Variablen eine Auswahl getroffen.

(a) Schwere des Ereignisses (event severity): Die Schwere der erfassten Ereignisse war in Unfall (crash), Beinaheunfall (near crash) sowie Baseline (Fahren ohne besondere Ereignisse) eingeteilt. Zum Zeitpunkt der Auswertung bestand der Datensatz aus 1.465 Unfällen, 2.710 Beinaheunfälle. und 32.586 Baselineabschnitten. Für die vorliegende Analyse waren ausschließlich die Daten der Baselineabschnitte von Interesse, da allgemein Bedingungen identifiziert werden sollten, die im Zusammenhang mit Texten (Schreiben und Lesen von Textnachrichten) oder Browsen stehen. Die Abschnitte des Fahrens in der Baselinebedingung wurden wiederum 2 Kategorien zugeordnet.

- balanced-sample baseline: Abschnitte, die vom gleichen Fahrer stammten, der einen Unfall oder Beinaheunfall hatte

- additional baseline: Abschnitte, die zufällig aus allen Fahrten aller Fahrten gezogen wurden

In die Auswertung wurden sowohl die balanced-sample baselines als auch die additional baselines einbezogen. Alle Baselineabschnitte waren 21 Sekunden lang.

(b) Nebentätigkeiten (secondary task): Für jeden Baselineabschnitt lagen im Datensatz Informationen zu während der Fahrt ausgeführten Nebentätigkeiten vor ${ }^{5}$. Von allen erfassten Nebentätigkeiten waren für die vorliegende Studie "Texten“ (texting) und „Browsen“ (browsing) relevant.

- Texten wurde definiert als das Drücken von Tasten oder Berühren des Touchscreens des Mobiltelefons durch den Fahrer, um eine Textnachricht zu verfassen bzw. zu senden.

- Browsen wurde beschrieben als das Drücken von Tasten oder Berühren des Touchscreens des Mobiltelefons durch den Fahrer, um das Internet oder Mobiltelefon-Anwendungen zu nutzen.

Texten und Browsen wurden für die folgenden Auswertungen zu „Manuelle Tätigkeiten am Mobiltelefon" zusammengefasst. Um zu ermitteln bei welchen Umgebungsbedingungen besonders häufig manuelle Tätigkeiten am Mobiltelefon

5 Es ist anzumerken, dass, abweichend von den sonstigen kodierten Variablen, für die Kodierung etwaiger Nebentätigkeiten nur die letzten 6 Sekunden des jeweiligen Baselineabschnitts betrachtet wurden. 
durchgeführt wurden, wurde die kodierte Kategorie „keine Nebenaufgaben“ zum Vergleich herangezogen (d.h. der Fahrer ist nur gefahren).

(c) Fahrerverhalten: Unter dieser Kategorie wurde die Variable „Fahrmanöver im betrachteten Abschnitt" (pre-incident maneuvre) berücksichtigt.

(d) Kontextvariablen ${ }^{6}$ : Hinsichtlich des Kontextes der Fahrsituation wurden folgende Variablen einer genauen Betrachtung unterzogen:

- Passagiere

- $\quad$ Anzahl der Passagiere auf dem Vordersitz (front seat passengers)

- $\quad$ Anzahl der Passagiere auf dem Rücksitz (rear seat passengers)

- Umwelteinflüsse

- $\quad$ Lichtverhältnisse (lighting)

- Witterungsbedingungen (weather)

- Zustand Fahrbahnoberfläche (surface conditions)

- Ort

- Ort (locality)

- Infrastrukturmerkmale

- Verkehrsführung (traffic flow)

- Anzahl der Fahrstreifen in die gleiche Richtung (through travel lanes)

- Kreuzungstypen (relation to junction)

- Verkehrsregelung (traffic control)

- Baustellenbereich (construction zone)

- Neigung (grade)

- Fahrtmerkmale

- Fahrtrichtung (alignment)

- Verkehrsdichte (traffic density)

\subsubsection{Datenanalyse}

Der Zugang zu den Daten erfolgte über die Website https://insight.shrp2nds.us/. Dort wurden über eine Datenbankabfrage einzelne Variablen ausgewählt und miteinander gekreuzt. Für die Baselineabschnitte wurden mittels Abfrage jeweils Texten und Browsen als Nebentätigkeit mit den zuvor beschriebenen Fahrerverhaltens- bzw. Kontextvariablen in Beziehung gesetzt. Die ermittelten Häufigkeiten für die Verhaltensweisen bzw. Kontextvariablen wurden dann in entsprechende Prozentanteile verrechnet (d.h. z.B. in wie viel Prozent aller Abschnitte, in denen keine Nebenaufgabe bearbeitet wurde, wurde

6 Die englischen Benennungen sind teilweise irreführend, und beschreiben die dahinterliegende Variable mitunter nur unzureichend. Bei der deutschen Benennung wurde auf eine präzisere Benennung geachtet, d.h. es handelt sich um keine direkte Übersetzung. 
eine bestimmte Kontextvariable beobachtet). Um klären zu können, ob bei Auftreten einer bestimmte Fahrverhaltensweise bzw. eines bestimmten Kontextmerkmals übermäßig häufig getextet bzw. gebrowst wurde, wurde danach das Odds Ratio (OR, auch Chancen- bzw. Quotenverhältnis) für Texting / Browsing unter den jeweiligen Bedingungen berechnet. (siehe Tabelle 2).

Tabelle 2: Kreuztabelle zur Berechnung der Odds Ratio.

\begin{tabular}{|l|c|c|}
\hline & $\begin{array}{c}\text { Potentieller Risikofaktor } \\
\text { präsent }\end{array}$ & $\begin{array}{c}\text { Potentieller Risikofaktor } \\
\text { abwesend }\end{array}$ \\
\hline Texting / Browsing-Fälle & $\mathrm{a}$ & $\mathrm{b}$ \\
\hline Kein Texting / Browsing & $\mathrm{c}$ & $\mathrm{d}$ \\
\hline
\end{tabular}

$$
O R=\frac{a / c}{b / d}
$$

Eine Odds Ratio über 1 bedeutet, dass bei Vorliegen der jeweiligen Verhaltensweise / des jeweiligen Kontexts von einer erhöhten "Chance“ für das Auftreten von Texting / Browsing ausgegangen werden kann. Ein Wert unter 1 bedeutet dagegen, dass bei Vorliegen der jeweiligen Verhaltensweise / des jeweiligen Kontexts die "Chance“ für Texting / Browsing reduziert ist.

\subsection{Ergebnisse der Analyse der Daten der SHRP2 NDS}

Insgesamt wurden 854 manuelle Tätigkeiten am Mobiltelefon beim Fahren in den Baselineabschnitten gefunden (2,8\%), wobei zwei Drittel dabei auf Texten $(n=603)$ und ein Drittel auf Browsen $(n=251)$ zurückgeführt werden konnte. Demgegenüber stehen 15.680 Abschnitte in denen keinerlei Nebentätigkeit beim Fahren ausgeführt wurde. Dies entspricht einem Anteil von 52,2\% an allen Baselineabschnitten.

\subsubsection{Fahrerverhalten}

Bei den Abschnitten mit manueller Tätigkeit am Mobiltelefon und den Abschnitten ohne Nebentätigkeit war der überwiegende Anteil aller Fahrmanöver ähnlich häufig zu beobachten (siehe Tabelle 3). Bei Fahrspurwechseln war allerdings eine reduzierte Bereitschaft $\mathrm{zu}$ Texten und Browsen erkennbar $(O R=0,46)$. Gleichzeitig ging ein plötzliches „driften“ beim Geradeausfahren mit verstärkter Telefonbedienung einher $(O R=6,45)$, was wohl eher als Folge der Bedienung zu interpretieren ist. 
Tabelle 3: Fahrerverhalten: Fahrmanöver im betrachteten Abschnitt (Pre-incident behaviour).

\begin{tabular}{|l|c|c|c|c|c|}
\hline Beschreibung & $\begin{array}{c}\text { Anzahl } \\
\text { manuelle } \\
\text { Tätigkeiten } \\
\text { am Mobil- } \\
\text { telefon }\end{array}$ & $\begin{array}{c}\text { \% Manuelle } \\
\text { Tätigkeiten } \\
\text { am Mobil- } \\
\text { telefon }\end{array}$ & $\begin{array}{c}\text { Anzahl } \\
\text { keine } \\
\text { Neben- } \\
\text { tätigkeiten }\end{array}$ & $\begin{array}{c}\text { \% keine } \\
\text { Neben- } \\
\text { tätigkeiten }\end{array}$ & OR \\
\hline $\begin{array}{l}\text { Geradeausfahren, konstante } \\
\text { Geschwindigkeit }\end{array}$ & 488 & 57,1 & 8.321 & 53,1 & 1,18 \\
\hline Abbremsen auf Fahrstreifen & 147 & 17,2 & 2.480 & 15,8 & 1,11 \\
\hline Eine Kurve fahren & 97 & 11,4 & 1.976 & 12,6 & 0,89 \\
\hline $\begin{array}{l}\text { Geradeausfahren, } \\
\text { Beschleunigen }\end{array}$ & 65 & 7,6 & 1.202 & 7,7 & 0,99 \\
\hline $\begin{array}{l}\text { Geradeausfahren, mit } \\
\text { unbeabsichtigtem "driften" } \\
\text { zwischen oder innerhalb der } \\
\text { Fahrstreifen }\end{array}$ & 28 & 3,3 & 82 & 0,5 & 6,45 \\
\hline Fahrstreifen wechseln & 14 & 1,6 & 543 & 3,5 & 0,46 \\
\hline
\end{tabular}

\subsubsection{Kontextvariablen}

Tabelle 4 gibt einen Überblick über die Anzahl der Passagiere auf Vorder- und Rücksitz. Es zeigte sich ein Zusammenhang zwischen der Häufigkeit der manuellen Bedienung des Mobiltelefons und der Präsenz von Passagieren. So scheint sich die Bereitschaft zur Beschäftigung mit Nebentätigkeiten bei Präsenz von Passagieren eher zu reduzieren.

Tabelle 4: Passagiere auf Vorder- und Rücksitz.

\begin{tabular}{|c|c|c|c|c|c|}
\hline Beschreibung & $\begin{array}{c}\text { Anzahl } \\
\text { manuelle } \\
\text { Tätigkeiten } \\
\text { am Mobil- } \\
\text { telefon }\end{array}$ & $\begin{array}{c}\text { \% Manuelle } \\
\text { Tätigkeiten } \\
\text { am Mobil- } \\
\text { telefon }\end{array}$ & $\begin{array}{c}\text { Anzahl } \\
\text { keine } \\
\text { Neben- } \\
\text { tätigkeiten }\end{array}$ & $\begin{array}{c}\text { \% keine } \\
\text { Neben- } \\
\text { tätigkeiten }\end{array}$ & OR \\
\hline \multicolumn{7}{|c|}{ Passagiere Vordersitz } \\
\hline $1^{8}$ & 755 & 88,4 & 12.406 & 79,1 & 2,01 \\
\hline 2 & 99 & 11,6 & 3.271 & 20,9 & 0,50 \\
\hline & \multicolumn{7}{|c|}{ Passagiere Rücksitz } \\
\hline 1 & 819 & 95,9 & 14.740 & 94,0 & 1,49 \\
\hline 2 & 24 & 2,8 & 620 & 3,9 & 0,70 \\
\hline & 10 & 1,2 & 260 & 1,7 & 0,70 \\
\hline
\end{tabular}

7 In der Ergebnistabelle sind jeweils nur die häufigsten Fahrverhaltensweisen bzw. Kontextvariablen berichtet. Entsprechend addieren sich die berichteten Prozentanteile nicht zu $100 \%$ auf.

$8 \quad 1$ Passagier $=$ Fahrer 
In Tabelle 5 sind die Häufigkeiten und Anteile der verschiedenen Umwelteinflüsse wie Lichtverhältnisse, Witterungsbedingungen und der Zustand der Fahrbahnoberfläche für die manuelle Bedienung am Mobiltelefon und die Abschnitte ohne Nebentätigkeit dargestellt. Auffällig ist lediglich, dass bei Dunkelheit, egal ob mit oder ohne Beleuchtung, häufiger getextet oder gebrowst wurde $(O R=1,62$ bzw. $O R=1,23)$. Für die Witterungs- und Oberflächenbedingungen wurde keine entsprechende Variation gefunden.

Tabelle 5: Umwelteinflüsse: Lichtverhältnisse, Witterungsbedingungen, Zustand Fahrbahnoberfläche.

\begin{tabular}{|c|c|c|c|c|c|}
\hline Beschreibung $^{9}$ & $\begin{array}{c}\text { Anzahl } \\
\text { manuelle } \\
\text { Tätigkeiten } \\
\text { am Mobil- } \\
\text { telefon }\end{array}$ & $\begin{array}{c}\text { \% Manuelle } \\
\text { Tätigkeiten } \\
\text { am Mobil- } \\
\text { telefon }\end{array}$ & $\begin{array}{l}\text { Anzahl } \\
\text { keine } \\
\text { Neben- } \\
\text { tätigkeiten }\end{array}$ & $\begin{array}{c}\% \text { keine } \\
\text { Neben- } \\
\text { tätigkeiten }\end{array}$ & OR \\
\hline \multicolumn{6}{|c|}{ Lichtverhältnisse } \\
\hline Tageslicht & 611 & 71,6 & 12.362 & 78,8 & 0,67 \\
\hline Dunkelheit, beleuchtet & 149 & 17,5 & 1.812 & 11,6 & 1,62 \\
\hline Dunkelheit, nicht beleuchtet & 60 & 7,0 & 905 & 5,8 & 1,23 \\
\hline \multicolumn{6}{|c|}{ Witterungsbedingungen } \\
\hline Keine ungünstigen Umstände & 774 & 90,6 & 14.049 & 89,6 & 1,12 \\
\hline Regen & 42 & 4,9 & 871 & 5,6 & 0,88 \\
\hline Nebel/ leichter Regen & 29 & 3,4 & 566 & 3,6 & 0,94 \\
\hline \multicolumn{6}{|c|}{ Zustand Fahrbahnoberfläche } \\
\hline Trocken & 714 & 83,6 & 13.014 & 83,0 & 1,04 \\
\hline Nass & 138 & 16,2 & 2.518 & 16,2 & 1,01 \\
\hline
\end{tabular}

Außerdem wurde betrachtet, inwieweit an bestimmten Örtlichkeiten oder Gebieten gehäuft manuelle Tätigkeiten am Mobiltelefon auftraten (siehe Tabelle 6). Hierbei zeigte sich, dass bei Schnellstraßen mit getrennten Fahrstreifen und Ampeln häufig am Mobiltelefon geschrieben oder gelesen wurde $(O R=1,32)$, ebenso wie im städtischen Bereich $(O R=1,52)$. Ebenso wurde eine erhöhte Quote an Schulen gefunden $(O R=1,16)$. An Kirchen oder auch in ländlichen Wohngebieten nutzten die Fahrer ihr Mobiltelefon dagegen seltener zum Texten oder Browsen $(O R=0,81$ bzw. $O R=0,71)$.

9 In der Ergebnistabelle sind jeweils nur die häufigsten Fahrverhaltensweisen bzw. Kontextvariablen berichtet. Entsprechend addieren sich die berichteten Prozentanteile nicht zu $100 \%$ auf. 
Tabelle 6: Ort.

\begin{tabular}{|l|c|c|c|c|c|}
\hline Beschreibung & $\begin{array}{c}\text { Anzahl } \\
\text { manuelle } \\
\text { Tätigkeiten } \\
\text { am Mobil- } \\
\text { telefon }\end{array}$ & $\begin{array}{c}\text { \% Manuelle } \\
\text { Tätigkeiten } \\
\text { am Mobil- } \\
\text { telefon }\end{array}$ & $\begin{array}{c}\text { Anzahl } \\
\text { keine } \\
\text { Neben- } \\
\text { tätigkeiten }\end{array}$ & $\begin{array}{c}\text { \% keine } \\
\text { Neben- } \\
\text { tätigkeiten }\end{array}$ & OR \\
\hline Industrie-/Gewerbegebiet & 275 & 32,2 & 5.140 & 32,8 & 0,97 \\
\hline $\begin{array}{l}\text { Autobahn/ Schnellstraße } \\
\text { getrennte Fahrbahnen ohne } \\
\text { Ampeln }\end{array}$ & 243 & 28,5 & 4.212 & 26,9 & 1,08 \\
\hline Städtische Wohngegend & 164 & 19,2 & 3.171 & 20,2 & 0,94 \\
\hline Schule & 52 & 6,1 & 831 & 5,3 & 1,16 \\
\hline $\begin{array}{l}\text { Schnellstraße getrennte } \\
\text { Fahrbahnen mit Ampeln }\end{array}$ & 40 & 4,7 & 563 & 3,6 & 1,32 \\
\hline Ländliche Wohngegend & 30 & 3,5 & 764 & 4,9 & 0,71 \\
\hline Städtisch & 25 & 2,9 & 306 & 2,0 & 1,52 \\
\hline Kirche & 15 & 1,8 & 339 & 2,2 & 0,81 \\
\hline
\end{tabular}

In Tabelle 7 sind die Häufigkeiten der Bedienung am Mobiltelefon für verschiedene Infrastrukturmerkmale dargestellt. Betrachtet man die Verkehrsführung, so wird deutlich, dass bei getrennten Fahrstreifen gehäuft getextet oder gebrowst wird ( $O R=1,31$ ), als wenn eine solche Trennung nicht vorliegt, oder es keine ausgewiesenen Fahrstreifen gibt. Bezogen auf die Anzahl der Fahrstreifen in die gleiche Richtung wurde bei Straßen ohne Fahrstreifen und mit fünf Fahrstreifen in gleicher Richtung eine geringere Rate manueller Tätigkeiten am Mobiltelefon beobachtet $(O R=0,56$ bzw. $O R=0,83)$. Auch bei den verschiedenen Kreuzungstypen wurden einzelne, unsystematische Unterschiede festgestellt. In Auf- / Abfahrten und an Verkehrsknotenpunkten wurden von den Fahrern häufiger manuelle Tätigkeiten am Mobiltelefon ausgeführt $(O R=1,26 \mathrm{bzw}$. $O R=1,15)$, während dies auf Parkplätzen seltener der Fall war $(O R=0,53)$. An Kreuzungen mit Stoppschildern wurde seltener getextet und gebrowst $(O R=0,69)$, während dies bei Abschnitten mit Baustellenschildern häufiger der Fall war $(O R=1,58)$. Ein ebenso uneinheitliches Bild zeigt sich, wenn man die Baustellensituationen genauer betrachtet. Bei der Einfahrt in eine Baustelle wurde weniger getextet $(O R=0,82)$, während sich die Rate in der Baustelle erhöhte $(O R=1,73)$. Auch wurde beim Fahren bergab tendenziell weniger getextet als im Schnitt $(O R=0,67)$.

10 In der Ergebnistabelle sind jeweils nur die häufigsten Fahrverhaltensweisen bzw. Kontextvariablen berichtet. Entsprechend addieren sich die berichteten Prozentanteile nicht zu $100 \%$ auf. 
Tabelle 7: Infrastrukturmerkmale: Verkehrsführung, Fahrstreifen, Kreuzungstypen, Verkehrsregelung, Baustellenbereich und Neigung.

\begin{tabular}{|c|c|c|c|c|c|}
\hline Beschreibung & $\begin{array}{c}\text { Anzahl } \\
\text { manuelle } \\
\text { Tätigkeiten } \\
\text { am Mobil- } \\
\text { telefon }\end{array}$ & $\begin{array}{c}\text { \% Manuelle } \\
\text { Tätigkeiten } \\
\text { am Mobil- } \\
\text { telefon }\end{array}$ & $\begin{array}{l}\text { Anzahl } \\
\text { keine } \\
\text { Neben- } \\
\text { tätigkeiten }\end{array}$ & $\begin{array}{c}\% \text { keine } \\
\text { Neben- } \\
\text { tätigkeiten }\end{array}$ & OR \\
\hline \multicolumn{6}{|c|}{ Verkehrsführung } \\
\hline $\begin{array}{l}\text { Geteilt (Mittelstreifen oder } \\
\text { Barriere) }\end{array}$ & 415 & 48,6 & 6.571 & 41,9 & 1,31 \\
\hline $\begin{array}{l}\text { Nicht geteilt - einfacher } \\
\text { Verkehrsweg in } 2 \text { Richtungen }\end{array}$ & 328 & 38,4 & 6.719 & 42,9 & 0,83 \\
\hline $\begin{array}{l}\text { Nicht geteilt - mittige 2- } \\
\text { Richtungen Linksabbiegespur }\end{array}$ & 66 & 7,7 & 1.341 & 8,6 & 0,90 \\
\hline Einbahnstraße & 30 & 3,5 & 543 & 3,5 & 1,01 \\
\hline Keine Fahrstreifen & 15 & 1,8 & 506 & 3,2 & 0,54 \\
\hline \multicolumn{6}{|c|}{ Fahrstreifen } \\
\hline 0 & 19 & 2,2 & 610 & 3,9 & 0,56 \\
\hline 1 & 315 & 36,9 & 6.280 & 40,1 & 0,87 \\
\hline 2 & 320 & 37,5 & 5.388 & 34,4 & 1,14 \\
\hline 3 & 151 & 17,7 & 2.378 & 15,2 & 1,20 \\
\hline 4 & 36 & 4,2 & 729 & 4,7 & 0,90 \\
\hline 5 & 12 & 1,4 & 265 & 1,7 & 0,83 \\
\hline \multicolumn{6}{|c|}{ Kreuzungstypen } \\
\hline Keine kreuzenden Straßen & 446 & 52,2 & 7.921 & 50,5 & 1,07 \\
\hline Kreuzung & 88 & 10,3 & 1.683 & 10,7 & 0,96 \\
\hline Autobahnkreuz & 87 & 10,2 & 1.404 & 9,0 & 1,15 \\
\hline Kreuzungsbezug & 82 & 9,6 & 1.521 & 9,7 & 0,99 \\
\hline Auffahrt, Zufahrtsweg, etc. & 69 & 8,1 & 1.222 & 7,8 & 1,04 \\
\hline Parkplatz Ein-/Ausfahrt & 46 & 5,4 & 1.125 & 7,2 & 0,74 \\
\hline Auffahrt/ Abfahrt & 23 & 2,7 & 337 & 2,2 & 1,26 \\
\hline Innerhalb des Parkplatzes & 13 & 1,5 & 448 & 2,9 & 0,53 \\
\hline
\end{tabular}




\begin{tabular}{|c|c|c|c|c|c|}
\hline Beschreibung & $\begin{array}{c}\text { Anzahl } \\
\text { manuelle } \\
\text { Tätigkeiten } \\
\text { am Mobil- } \\
\text { telefon }\end{array}$ & $\begin{array}{c}\% \text { Manuelle } \\
\text { Tätigkeiten } \\
\text { am Mobil- } \\
\text { telefon }\end{array}$ & $\begin{array}{l}\text { Anzahl } \\
\text { keine } \\
\text { Neben- } \\
\text { tätigkeiten }\end{array}$ & $\begin{array}{c}\% \text { keine } \\
\text { Neben- } \\
\text { tätigkeiten }\end{array}$ & OR \\
\hline \multicolumn{6}{|c|}{ Verkehrsregelung $^{11}$} \\
\hline Keine Verkehrsregelung & 711 & 83,3 & 13.018 & 83,0 & 1,02 \\
\hline Ampel & 58 & 6,8 & 1008 & 6,4 & 1,06 \\
\hline Baustellenschilder/ Warnungen & 35 & 4,1 & 414 & 2,6 & 1,58 \\
\hline Stoppschild & 17 & 2,0 & 449 & 2,9 & 0,69 \\
\hline \multicolumn{6}{|c|}{ Baustellenbereich } \\
\hline $\begin{array}{l}\text { Nicht } \\
\text { Baustellenbereichsbezogen }\end{array}$ & 812 & 95,1 & 15.100 & 96,3 & 0,74 \\
\hline $\begin{array}{l}\text { Baustellenbereich (Ereignis im } \\
\text { Bereich) }\end{array}$ & 31 & 3,6 & 334 & 2,1 & 1,73 \\
\hline $\begin{array}{l}\text { Auf Baustellenbereich bezogen } \\
\text { (Ereignis bei Annäherung an } \\
\text { Bereich oder anderweitig auf } \\
\text { Bereich bezogen) }\end{array}$ & 11 & 1,3 & 245 & 1,6 & 0,82 \\
\hline \multicolumn{6}{|c|}{ Neigung } \\
\hline Eben & 735 & 86,1 & 13.415 & 85,6 & 1,04 \\
\hline Anstieg & 83 & 9,7 & 1.463 & 9,3 & 1,05 \\
\hline Gefälle & 26 & 3,0 & 703 & 4,5 & 0,67 \\
\hline
\end{tabular}

Auch für die Fahrtrichtung ergaben sich nur wenige nennenswerte Unterschiede (siehe Tabelle 8). Bei Rechtskurven war eine Reduktion manueller Tätigkeiten am Mobiltelefon zu beobachten $(O R=0,72)$. Bei Stau und stockendem Verkehr wurden mehr manuelle Tätigkeiten am Mobiltelefon vorgenommen $(O R=1,61)$, während dies bei freier Fahrt seltener vorkam $(O R=0,83)$. Andere Variablen hatten keinen Einfluss.

Tabelle 8: Fahrtmerkmale: Fahrtrichtung und Verkehrsdichte.

\begin{tabular}{|l|c|c|c|c|c|}
\hline Beschreibung & $\begin{array}{c}\text { Anzahl } \\
\text { manuelle } \\
\text { Tätigkeiten } \\
\text { am Mobil- } \\
\text { telefon }\end{array}$ & $\begin{array}{c}\% \text { Manuelle } \\
\text { Tätigkeiten } \\
\text { am Mobil- } \\
\text { telefon }\end{array}$ & $\begin{array}{c}\text { Anzahl } \\
\text { keine } \\
\text { Neben- } \\
\text { tätigkeiten }\end{array}$ & $\begin{array}{c}\text { \% keine } \\
\text { Neben- } \\
\text { tätigkeiten }\end{array}$ & OR \\
\hline \multicolumn{7}{|c|}{ Fahrtrichtung } \\
\hline Geradeaus & 741 & 86,8 & 13.338 & 85,1 & 1,15 \\
\hline Linkskurve & 64 & 7,5 & 1.116 & 7,1 & 1,06 \\
\hline Rechtskurve & 49 & 5,7 & 1.226 & 7,8 & 0,72 \\
\hline
\end{tabular}

11 In der Ergebnistabelle sind jeweils nur die häufigsten Fahrverhaltensweisen bzw. Kontextvariablen berichtet. Entsprechend addieren sich die berichteten Prozentanteile nicht zu $100 \%$ auf. 


\begin{tabular}{|l|c|c|c|c|c|}
\hline & \multicolumn{1}{|c|}{$\begin{array}{c}\text { Anzahl } \\
\text { manuelle } \\
\text { Tätigkeiten } \\
\text { am Mobil- } \\
\text { telefon }\end{array}$} & $\begin{array}{c}\text { \% Manuelle } \\
\text { Tätigkeiten } \\
\text { am Mobil- } \\
\text { telefon }\end{array}$ & $\begin{array}{c}\text { Anzahl } \\
\text { keine } \\
\text { Neben- } \\
\text { tätigkeiten }\end{array}$ & $\begin{array}{c}\text { \% keine } \\
\text { Neben- } \\
\text { tätigkeiten }\end{array}$ & OR \\
\hline \multicolumn{2}{|c|}{ Verkehrsdichte } & \\
\hline $\begin{array}{l}\text { Freier Verkehrsfluss, keine } \\
\text { Vorausfahrenden }\end{array}$ & 283 & 33,1 & 5.867 & 37,4 & 0,83 \\
\hline $\begin{array}{l}\text { Freier Verkehrsfluss, } \\
\text { Vorausfahrende }\end{array}$ & 286 & 33,5 & 4.796 & 30,6 & 1,14 \\
\hline $\begin{array}{l}\text { Verkehrsfluss mit leichten } \\
\text { Einschränkungen }\end{array}$ & 226 & 26,5 & 4.054 & 25,9 & 1,03 \\
\hline $\begin{array}{l}\text { Stabiler Verkehrsfluss, } \\
\text { Manövrierbarkeit und } \\
\text { Geschwindigkeit eingeschränkt }\end{array}$ & 33 & 3,9 & 664 & 4,2 & 0,91 \\
\hline $\begin{array}{l}\text { Unbeständiger Verkehrsfluss - } \\
\text { vorübergehende } \\
\text { Einschränkungen verlangsamen } \\
\text { Fahrer erheblich }\end{array}$ & 16 & 1,9 & 184 & 1,2 & 1,61 \\
\hline
\end{tabular}

\subsection{Zusammenfassung}

Gemessen an der Gesamtzahl der untersuchten Abschnitte wurde das Schreiben von Textnachrichten bzw. das Browsen am Mobiltelefon selten beobachtet. Für den Einfluss der einzelnen Fahrverhaltens- und Kontextvariablen zeigte sich allerdings ein sehr diverses Bild. Für viele Variablen wurden erwartungsgemäße Ergebnisse gefunden, so z.B. dass Passagiere die Neigung zum Texten oder Browsen verringern, ebenso wie schwierige Fahrmanöver, enge Straßen ohne eindeutige Fahrpuren oder ein Stoppschild, bei dem sich die Fahrer orientieren müssen. Bei diesen eher komplexen Situationen bzw. Situationen, die erhöhte Aufmerksamkeit des Fahrers benötigen, erscheint es nur folgerichtig, dass die Fahrer weniger häufig zum Mobiltelefon griffen. Auch für die verstärkte Nutzung des Mobiltelefons zum Texten oder Browsen wurden zum Teil erwartungsgemäße Ergebnisse gefunden, wie etwa, dass Fahrer häufiger im Stau bzw. stockenden Verkehr texten oder in der Nacht, wenn wenig Verkehr ist. Diese Ergebnisse legen nahe, dass der Kontext bei der Bearbeitung von Nebentätigkeiten durchaus eine Rolle spielt.

Gleichzeitig besteht ein starker Zusammenhang zwischen verschiedenen Kontextvariablen, so dass konkrete Ursachenzuschreibungen auf Basis dieser Daten nur schwer möglich sind. Exemplarisch lässt sich das an dem Befund, dass in der Nähe von Schulen häufiger getextet wurde, beschreiben. Da an Schulen viele überquerende, u.U. wenig vorhersehbare Fußgänger (Kinder) zu erwarten sind, wäre eigentlich von einer erhöhten Komplexität der Situation, und damit von einer reduzierten Mobiltelefonnutzung auszugehen. Gleichzeitig kann aber auch vermutet werden, dass es sich bei den Fahrern in einigen Fällen um Eltern handelt, die ihre Kinder eben von jener Schule abholen, oder sie gerade dort abgesetzt haben, und diese Tatsache einen konkreten

12 In der Ergebnistabelle sind jeweils nur die häufigsten Fahrverhaltensweisen bzw. Kontextvariablen berichtet. Entsprechend addieren sich die berichteten Prozentanteile nicht zu $100 \%$ auf. 
Anlass für das Schreiben einer Nachricht liefert (z.B. um dem Partner mitzuteilen, dass man sich auf dem Heimweg befindet). An diesem Beispiel wird deutlich, dass Erklärungen für den Einfluss einzelner Faktoren eher schwierig sind, vor allem, wenn keine Kontrolle über die Motive für das Verfassen von Textnachrichten besteht. Es ist davon auszugehen, dass die „Notwendigkeit“ zu texten sehr wahrscheinlich nicht gleichmäßig über eine Fahrt verteilt ist. Denkbar ist, dass z.B. eher zu Fahrtbeginn getextet wird (z. B. weil zuvor begonnene Nachrichten oder "Dialoge“ noch beendet werden). Gleichzeitig gibt es wiederum einen Zusammenhang zwischen bestimmten Abschnitten der Fahrt und den Kontextvariablen, z.B. Fahrtbeginn häufig im städtischen Bereich, in der Nähe bestimmter Lokalitäten.

Ebenso problematisch ist die Verknüpfung von Fahrverhaltensvariablen und der Bedienung des Mobiltelefons. So ist bei einigen der kodierten Ausprägungen des Fahrverhaltens davon auszugehen, dass es sich nicht um ein dauerhaftes Verhalten handelt, vor dessen Hintergrund eine Entscheidung für oder gegen das Texten getroffen wird, sondern vielmehr um ein Verhalten als Folge des Textens, z.B. eine stärkere Variation in der Spurhaltung. Auch muss berücksichtigt werden, dass nicht alle Kontexte vollkommen auf den deutschen Verkehr übertragbar sind. Deutlich wird dies z.B. bei der Anzahl der Fahrstreifen, wo davon auszugehen ist, dass Straßen mit 5 Fahrstreifen in die gleiche Richtung in Deutschland eher selten anzutreffen sind, ebenso wie sehr breite Fahrbahnen. Auch die Tatsache, dass in Deutschland, anders als den USA, immer noch weitestgehend mit manuellem Getriebe gefahren wird, kann zu Abweichungen führen, wenn man bedenkt, dass sich die Notwendigkeit zu Schalten (und damit beide Hände zur Bewältigung der Fahraufgabe einzusetzen) nicht gleichmäßig über alle Kontexte verteilt. Aus diesen Gründen können die SHRP2 Daten zwar wertvolle Hinweise für die generelle Rolle von Kontextfaktoren für die Bereitschaft zur Bearbeitung von Nebenaufgaben liefern, können aber im Detail nicht aufklären, welche Faktoren speziell im Raum Deutschland gegebenenfalls mit einer erhöhten Nutzungsbereitschaft einhergehen. 


\section{Videobasierte, strukturierte Interviewstudie zur Identifikation von Kontexten in denen Fahrer Textnachrichten verfassen}

Aus der Analyse der SHRP2 Daten wurde bereits deutlich ist, dass anscheinend übergeordnete Faktoren, wie etwa die Komplexität der Verkehrssituation, bei der Entscheidung eine Textnachricht zu schreiben eine Rolle spielen. Entsprechend sollte sich die in der Folge beschriebene videobasierte, strukturierte Interviewstudie mit dem Einfluss, dieser und anderer Faktoren, beschäftigen. Für Fastenmeier (1995) setzt sich die Komplexität einer Verkehrssituation aus den Anforderungen an die Informationsverarbeitung des Fahrers und den Anforderungen zusammen, die sich aus der Fahrzeugbedienung ergeben. Zur Bestimmung der Aufgabenkomplexität von Verkehrssituationen steht von Fastenmeier (1995) eine umfassende Klassifikation zur Verfügung. Diese bezieht die Dauer der Situation, das Vorhandensein von Kreuzungen, die Art der Knotenpunktregelung, die Sicht- und Wetterbedingungen und die Verkehrsdichte mit ein. Beispiele für wenig komplexe Situationen sind lang andauernde Situationen auf gerader Strecke ohne Knotenpunkte, z.B. auf der Autobahn oder Landstraße, definiert. Als schwierige Situationen gelten z.B. kurze innerstädtische Situationen an Knotenpunkten, bei denen der Fahrer an eine Wartepflicht gebunden ist, und damit entsprechend auch andere Verkehrsteilnehmer zu beachten hat. Um den Einfluss der Komplexität der Verkehrssituation auf das Verfassen von Textnachrichten zu untersuchen, wurden Autofahrern Videos von verschieden komplexen Verkehrssituationen präsentiert. Zum einen sollten sie diese dahingehend bewerten, ob sie eine Textnachricht schreiben würden. Zum anderen wurde ein strukturiertes Interview zu Merkmalen der Situation durchgeführt. Zusätzlich zur generellen Variation der Komplexität wurden auch verschiedene Verkehrsumgebungen (Autobahn, Landstraße, Stadtverkehr) und damit Geschwindigkeitsbereiche gezeigt. Das Ziel dieser Teilstudie bestand darin, aufbauend auf den Erkenntnissen der SHRP2 Analyse, Kontextfaktoren (Infrastruktur, Verkehrsumgebung etc.) systematisch und detailliert herauszuarbeiten, unter denen die Autofahrer bereit, oder auch nicht bereit sind, Textnachrichten zu verfassen.

\subsection{Methode}

\subsubsection{Stichprobe}

Die Rekrutierung der Teilnehmer erfolgte über eine Auswahl aus der Probandendatenbank der Professur für Allgemeine Psychologie und Arbeitspsychologie sowie Aufrufe über Mailverteiler der Technischen Universität Chemnitz. Besonderes Augenmerk wurde dabei auf die Ansprache von Autofahrern mit hoher jährlicher Fahrleistung gelegt. Außerdem sollten Personen angeworben werden, die grundsätzlich bereit sind ihr Mobiltelefon während der Fahrt für Textnachrichten zu nutzen.

Die Auswahl der Teilnehmer erfolgte über einen Fragebogen zur Kontaktaufnahme, welcher als Onlinebewerbungstool an 180 Personen verschickt wurde. Der Fragebogen enthielt u.a. Angaben zu soziodemografischen Variablen (Alter, Geschlecht), zum Fahrzeug (Art des Fahrzeugs, Kilometerleistung) sowie zum Mobiltelefon und dessen Nutzung (Art des Telefons, Nutzungshäufigkeit beim Fahren). Insgesamt bewarben sich 71 Personen für die Teilnahme an der Studie, deren Zahl in einem Auswahlprozess sukzessive reduziert wurde. Bei der Selektion wurden folgende Kriterien berücksichtigt:

- mindestens 12.000 km Fahrleistung im Jahr

- mindestens 1 verfasste Textnachricht je 10 Stunden Fahrzeit 
Es wurden 41 Autofahrer (19 Frauen, 22 Männer) mit einem Durchschnittsalter von 32,7 Jahren (19 bis 63 Jahre) für die Teilnahme ausgewählt. Die Teilnehmer besaßen den Führerschein der Klasse B (Pkw) im Schnitt seit 14,5 Jahren (2 bis 43 Jahre). Die durchschnittliche jährliche Fahrleistung betrug $30.146 \mathrm{~km}$ (12.000 bis $95.000 \mathrm{~km}$ ). Die Mehrheit fuhr die meiste Zeit einen Pkw $(n=37)$, nur drei Teilnehmer fuhren überwiegend Kleintransporter, ein weiterer primär einen Lkw. 40 Teilnehmer nutzten ein Smartphone, nur einer ein konventionelles Mobiltelefon (Verteilung auf Typen siehe Tabelle 9). Fast zwei Drittel der Teilnehmer waren an das Betriebssystem Android gewöhnt (61\%), nahezu ein Drittel an iOS (32\%). Nur wenige nutzten andere Betriebssysteme (7\%). Zum Verfassen von persönlichen Nachrichten gaben beinahe drei Viertel der Teilnehmer an, üblicherweise WhatsApp (73\%) zu nutzen. SMS (14\%) und Facebook (8\%) wurden deutlich seltener genutzt, ebenso wie sonstige Dienste $(5 \%)$.

Tabelle 9: Überblick über die genutzten Mobiltelefone $\left(n=42^{13}\right)$.

\begin{tabular}{|l|c|c|}
\hline Art Mobiltelefon & Häufigkeit & Prozent \\
\hline Samsung & 18 & 42,9 \\
\hline Apple & 14 & 33,3 \\
\hline Sony & 5 & 11,9 \\
\hline HTC & 2 & 4,8 \\
\hline LG & 1 & 2,4 \\
\hline Motorola & 1 & 2,4 \\
\hline Nokia & 1 & 2,4 \\
\hline
\end{tabular}

\subsubsection{Material}

Mit den Teilnehmern wurden videogestützte Interviews durchgeführt. Hierfür wurden Videos von verschiedenartigen Verkehrssituationen in diversen Kontexten aufgenommen. Insgesamt wurden 43 Situationen ausgewählt, wobei explizit keine kritischen Situationen enthalten waren (z.B. Kreuzungssituationen in denen andere Autofahrer die Vorfahrt nicht beachten). Es sollte das alltägliche unkritische Fahren abgebildet werden. Die Auswahl der Szenen erfolgte anhand der verschiedenen Verkehrsumgebungen (Autobahn, Landstraße, Stadtverkehr) und der Komplexität der Verkehrssituation. Es wurden 14 Szenen auf der Autobahn, 8 Szenen auf der Landstraße und 21 Szenen aus dem innerstädtischen Bereich ausgewählt. Nach Fastenmeier (1995) ließen sich davon 9 Situationen als gering komplex, 9 Situationen als durchschnittlich komplex, und 8 Situationen als hoch komplex einstufen. Die siebzehn übrigen Situationen wurden in die videobasierte, strukturierte Interviewstudie aufgenommen, da sie relevante Sondersituationen für Autofahrer darstellen, und häufig im Straßenverkehr vorkommen (z.B. Tunnelfahrt, Überholen eines Radfahrers oder Fahren in einer 30er Zone). Diese Situationen konnten nicht unmittelbar einer Komplexitätsstufe zugeordnet werden, da sie nicht dem Schema von Fastenmeier (1995) entsprachen.

Das Videomaterial wurde mit einer Auflösung von $1.920 \times 1.080$ mit einer Bildrate von 25 Bildern pro Sekunde im alltäglichen Straßenverkehr aufgezeichnet. Die geschnittenen

13 Ein Teilnehmer besaß 2 Primärtelefone (Dienst- und Privattelefon), weshalb sich die Gesamtzahl erhöhte. 
Sequenzen hatten jeweils eine Länge zwischen 6 und 18 Sekunden. Um den Teilnehmer zusätzlich Informationen über die Fahrsituation zu geben, wurde die jeweils gefahrene Geschwindigkeit im Video angezeigt (siehe Abbildung 2).

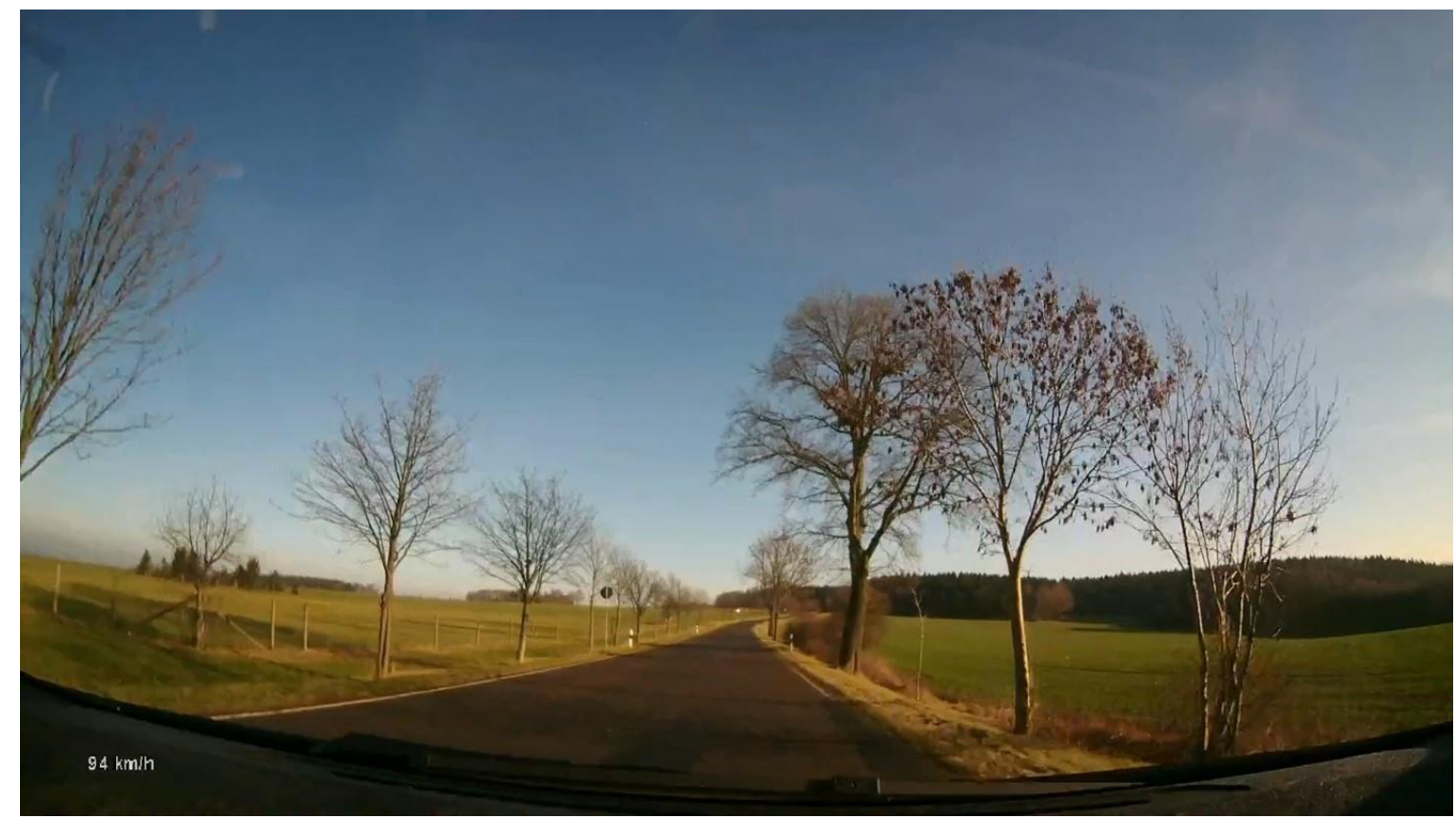

Abbildung 2: Videoausschnitt für eine Situation auf der Landstraße.

Ferner wurde ein Fragebogen zur Erfassung von Persönlichkeitsmerkmalen eingesetzt. Dieser beinhaltete u.a. Skalen, die die Einstellung gegenüber dem Schreiben von Textnachrichten (Harrison, 2011) sowie das gewohnheitsmäßige Schreiben von Textnachrichten (Bayer \& Campbell, 2012) erfassten. Außerdem wurden in Anlehnung an die Theorie des geplanten Verhaltens die subjektive Norm, Einstellung und Verhaltenskontrolle über das Texten während des Fahrens erhoben (Bayer \& Campbell, 2012). Als fahrbezogene Variable wurde der Fahrstil der Teilnehmer abgefragt (Popken, 2009).

\subsubsection{Ablauf}

Zu Beginn des Interviews erhielten die Teilnehmer eine kurze Einführung zur Studie durch den Versuchsleiter, sowie eine Aufklärung, dass Video- und Audiodaten aufgezeichnet werden. Danach sollten sie eine standardisierte Textnachricht an ihrem Mobiltelefon schreiben, wobei die Eingabe gefilmt wurde um verschiedene Eingabestrategien (z.B. Eingabe mit dem Daumen) zu erfassen.

Anschließend erhielten die Teilnehmer die Instruktionen für das Interview. Innen wurde mitgeteilt, dass sie in der Folge verschiedene Verkehrssituationen aus der Fahrerperspektive zu sehen bekämen, bei denen sie sich in die Rolle des Fahrers hineinversetzen sollten. Die Aufgabe der Teilnehmer bestand darin zu entscheiden, ob sie unter den dargestellten Rahmenbedingungen bereit wären, eine Textnachricht zu schreiben (unabhängig von der dafür genutzten Applikation). Dabei wäre es der Teilnehmer, der die „Unterhaltung“ initiiert, d.h. die Textnachricht sollte keine Antwort auf eine zuvor erhaltene Nachricht darstellen. Wenn die Teilnehmer ihre Bereitschaft zum Verfassen einer Textnachricht äußerten, sollten sie anschließend begründen, warum sie mit dem Schreiben einer Textnachricht beginnen würden, bzw. bei Ablehnung, warum sie das nicht tun würden. Dabei wurden sie darauf hingewiesen, dass sie bei ihrer 
Begründung nur Merkmale der Situation nennen, und keine pauschalen Aussagen wie „weil mir jemand schreibt" treffen sollten. Danach wurden die Teilnehmer noch einmal komplementär zur bisherigen Begründung gefragt, was in der jeweiligen Situation anders sein müsste, damit sie das Verfassen einer Textnachricht unterlassen (wenn sie zuvor angegeben hatten, dass sie in der Situation texten würden), bzw. was anders sein müsste, dass sie mit dem Verfassen einer Textnachricht beginnen (wenn sie zuvor angegeben hatten, dies nicht zu tun). Sie sollten dabei lediglich ein, das entscheidendste Merkmal benennen. Anschließend sollten sie die Erhöhung des Unfallrisikos bewerten, die sich ergäbe, wenn sie in der Situation mit dem Schreiben einer Textnachricht begonnen hätten (vgl. Interviewleitfaden im Anhang).

Nach Abschluss der Instruktionen wurden den Teilnehmern die 43 Situationen dargeboten. Um Reihenfolgeeffekte auszuschließen wurden 5 Abfolgen der Situationen erstellt. Diese unterschiedlichen Abfolgen wurden über die Teilnehmer hinweg ausbalanciert. Zu den Videos mussten sie jeweils die zuvor beschriebenen Fragen beantworteten. Der Versuchsleiter wurde instruiert nachzufragen, wenn die Teilnehmer nur pauschale, nicht auf die Situation bezogene Aussagen trafen. Im Anschluss an das Interview füllten die Teilnehmer den Fragebogen aus, und wurden danach verabschiedet. Insgesamt dauerte das Interview im Schnitt 60 Minuten (zwischen 35 und 90 Minuten).

\subsubsection{Datenaufbereitung und -analyse}

Insgesamt wurden ca. 3.000 Minuten Interviewmaterial aufgezeichnet. Die Audioaufnahmen wurden vollständig nachgehört und einzelne, wortwörtliche Aussagen der Personen transkribiert. Für die Frage „Warum würden Sie in dieser Situation mit dem Schreiben beginnen bzw. nicht beginnen?" wurden insgesamt 1.996 Argumente dafür gesammelt, dass man in der jeweiligen Situation eine Textnachricht verfassen würde, sowie 2.648 Argumente dafür das Schreiben zu unterlassen. Für die Auswertung wurde eine qualitative Inhaltsanalyse nach Mayring (2000) durchgeführt. Hierfür wurden die Argumente zu größeren Kategorien in mehreren Einzelschritten zusammengefasst, wobei die Kategorien wiederholt überarbeitet und verdichtet wurden. Diese Kategorien wurden anschließend bezogen auf die Häufigkeit der Nennungen ausgewertet. Das gleiche Vorgehen wurde bei der zweiten offenen Frage angewandt (was müsste anders sein, damit die Teilnehmer texten / nicht texten). Für die Frage nach der Unterlassung wurden 1.256 Argumente ausgewertet, sowie für die Frage „Was müsste anders sein, dass Sie texten?" 1.497 Argumente.

Die Angaben aus dem Fragebogen zur Kontaktaufnahme und der Befragung nach dem Interview wurden ebenfalls aufbereitet und auf ihre Plausibilität überprüft. Die Videos zur Eingabe der Textnachricht am Smartphone wurden alle gesichtet und verschiedene Eingabestrategien der Teilnehmer frei beschrieben. Diese Strategien wurden in einem zweiten Schritt zu Kategorien (z.B. „tippt mit beiden Daumen“) zusammengefasst und dann bezogen auf die Häufigkeit des Auftretens ausgewertet. 


\subsection{Ergebnisse}

\subsubsection{Häufigkeit des Lesens und Schreibens von Textnachrichten aus Fragebogendaten}

In Abbildung 3 ist die Häufigkeit des Lesens bzw. die Nutzung der Vorlesefunktion für Textnachrichten gegenübergestellt. Es ist erkennbar, dass die Teilnehmer eher dazu tendierten, Textnachrichten manuell am Display ihres Mobiltelefons zu lesen, während die Vorlesefunktion kaum genutzt wurde.

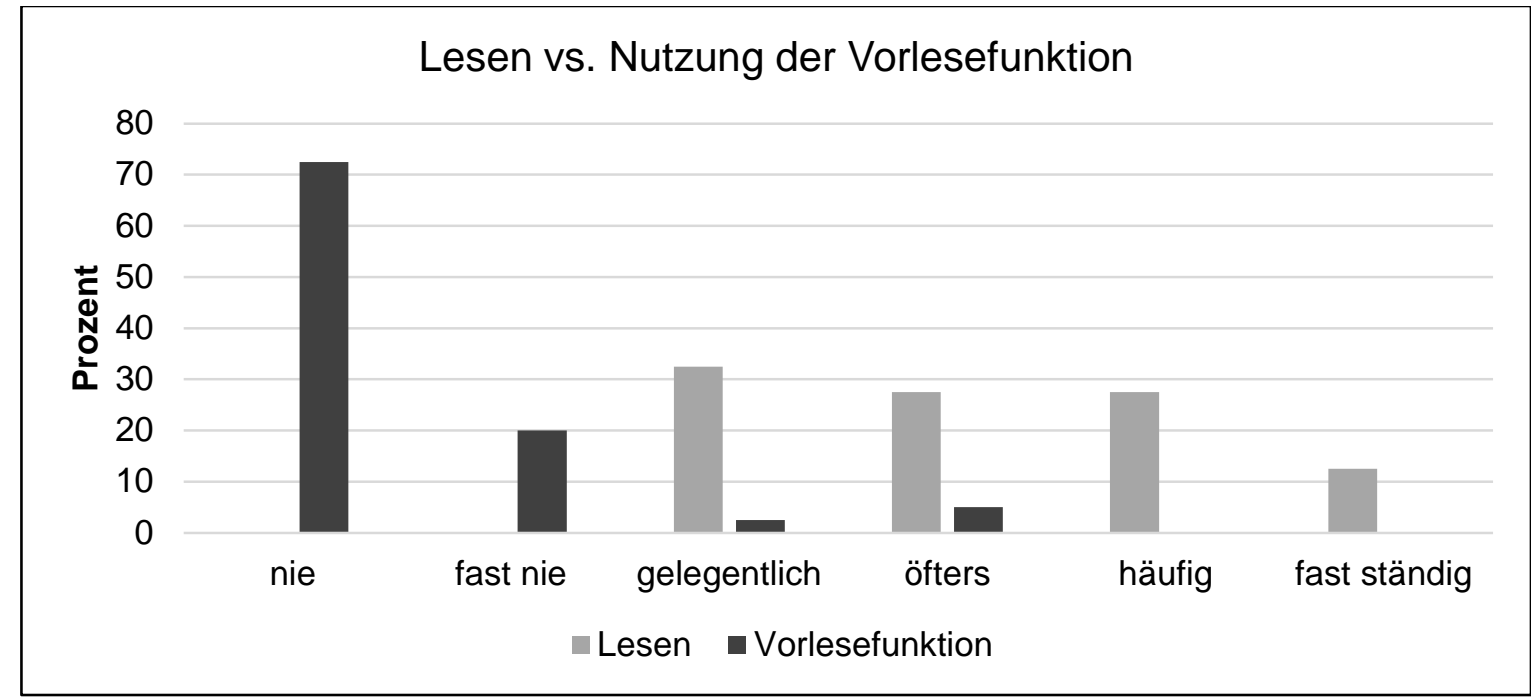

Abbildung 3: Häufigkeit des Lesens von Textnachrichten bzw. der Nutzung Vorlesefunktion während des Fahrens, $n=40$.

Für das Schreiben von Textnachrichten am Mobiltelefon ist ein ähnliches, wenn auch nicht ganz so deutliches, Muster erkennbar. Während die meisten Teilnehmer angaben, zumindest gelegentlich manuelle Eingaben zu tätigen, wird die Spracheingabe eher selten genutzt (siehe Abbildung 4).

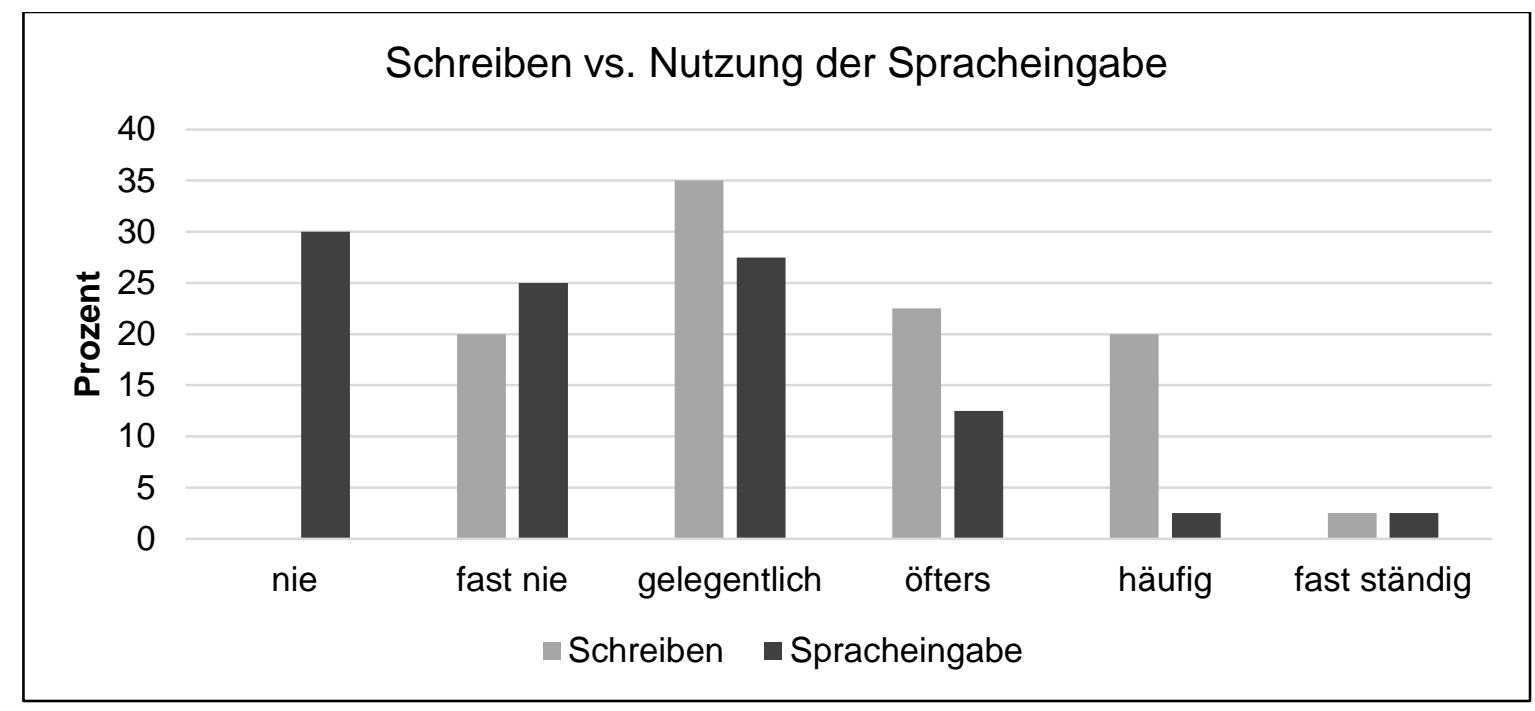

Abbildung 4: Häufigkeit des Schreibens von Textnachrichten bzw. der Nutzung der Spracheingabe während des Fahrens, $n=40$. 
Aus einer präzisierenden Frage zur Nutzungshäufigkeit in Relation zur Fahrzeit lässt sich bestätigen, dass alle Teilnehmer eine generelle Bereitschaft zum Bearbeiten von Textnachrichten aufwiesen, wobei Lesen häufiger als Schreiben berichtet wurde (siehe Abbildung 5). Entsprechend kann davon ausgegangen werden, dass die Teilnehmer an der Untersuchung grundsätzlich geübt darin waren, während des Fahrens Textnachrichten zu lesen und zu schreiben.

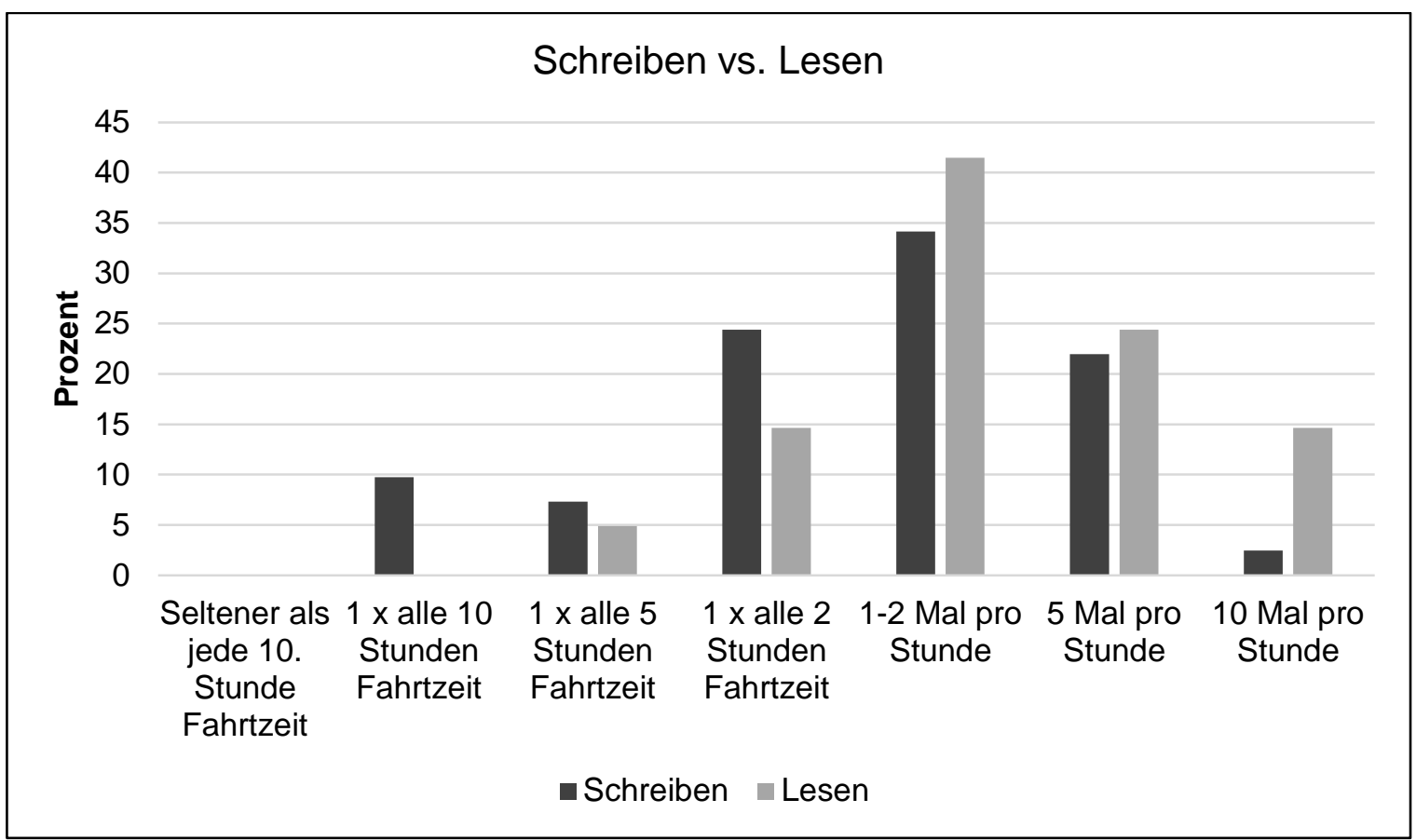

Abbildung 5: Häufigkeit des Schreibens von Textnachrichten bzw. der Nutzung der Spracheingabe während des Fahrens $(N=41)$.

\subsubsection{Eingabestrategien bei der Eingabe einer Textnachricht}

Der überwiegende Teil der Teilnehmer tippte die Textnachricht mit dem rechten Daumen (59\%), während $22 \%$ den rechten Zeigefinger benutzten und $12 \%$ beide Daumen. Nur ein geringer Teil bediente das Mobiltelefon mit der linken Hand (7\%). Außerdem tippte knapp die Hälfte der Teilnehmer jeden Buchstaben einzeln (49\%). Die Wortvervollständigung wurde von $44 \%$ der Teilnehmer genutzt, während $7 \%$ die SwipeFunktion nutzten (zur Worteingabe wird über die Tastatur gewischt). 


\subsubsection{Häufigkeit des Verfassens von Textnachrichten in den gezeigten Verkehrs- situationen}

Im Schnitt würden die Teilnehmer in 18,5 der 43 präsentierten Situationen eine Textnachricht schreiben $(43 \%, S D=6,5)$. Der vorsichtigste Teilnehmer würde dabei in nur sechs Situationen eine Textnachricht verfassen, während am anderen Ende des Spektrums zwei Teilnehmer bei 34 Situationen schreiben würden. In Abbildung 6 ist zu erkennen, dass die meisten Teilnehmer in 14 bis 22 Situationen Textnachrichten schreiben würden.

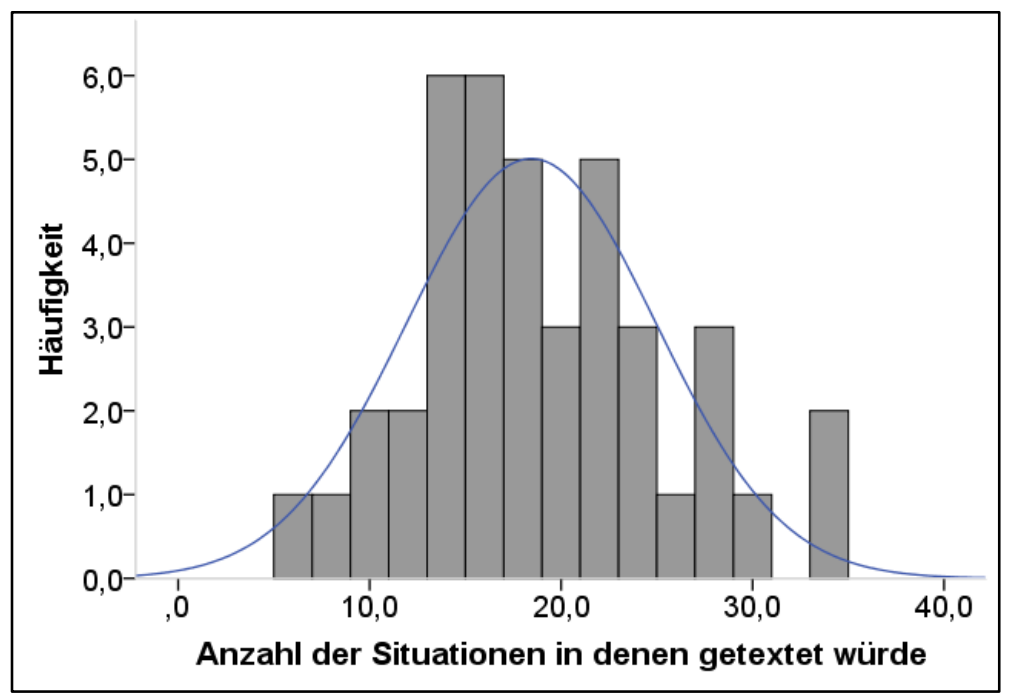

Abbildung 6: Häufigkeit der Anzahl der Situationen in denen die Teilnehmer eine Textnachricht schreiben würden $(N=41)$.

Ferner gab es Situationen, in denen beinahe jeder Teilnehmer (98\%) eine Textnachricht schreiben würde (z.B. stehend an der roten Ampel, siehe Abbildung 7 links) und auch Situationen in denen kein Teilnehmer eine Textnachricht schreiben würde (z.B. kurvige Landstraße, siehe Abbildung 7 rechts). Insgesamt war die Situationsauswahl sehr ausgewogen, wie sich an der Verteilung der Häufigkeit des Schreibens zeigt. Bei 18 Situationen würden jeweils zwischen $0 \%$ und $33 \%$ der Teilnehmer texten, bei 14 Situationen waren es zwischen $34 \%$ und $66 \%$ der Teilnehmer, und bei 11 Situationen waren es $67 \%$ bis $98 \%$ der Teilnehmer.
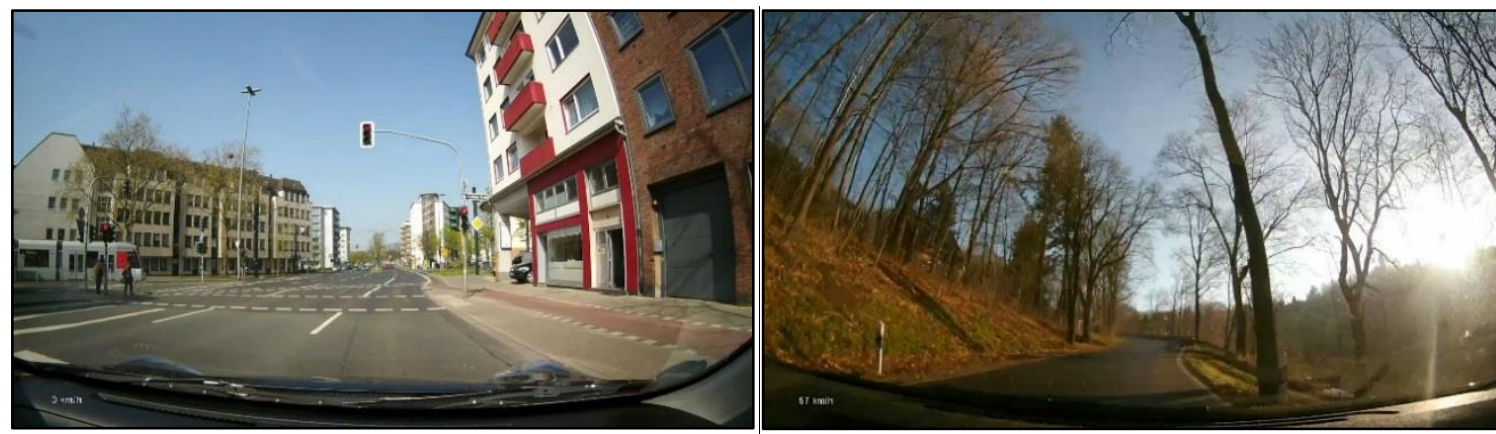

Abbildung 7: Links, eine Situation in der beinahe alle Teilnehmer eine Textnachricht verfasst hätten; rechts, eine Situation in der niemand eine Textnachricht verfasst hätte.

Hinsichtlich der Komplexität der Situationen zeigte sich, dass bei Situationen die von vornherein als hoch komplex eingestuft wurden, im Schnitt nur etwa ein Fünftel der Teilnehmer eine Textnachricht schreiben würde (siehe Tabelle 10), während es bei Situationen von mittlerer Komplexität immerhin ca. $40 \%$ und bei geringer Komplexität 
sogar über $60 \%$ waren. Bei den Situationen, die nicht in das Komplexitätsschema passten, wurde anscheinend eine ausgewogene Mischung gefunden. Im Mittel würden knapp $45 \%$ der Teilnehmer in diesen Situationen eine Textnachricht schreiben. Das Minimum lag bei $2 \%$ der Teilnehmer (Innenstadt: Radfahrer fährt vor Autofahrer, zweispurige Straße mit Gegenverkehr) und das Maximum bei 98\% der Teilnehmer (warten an der roten Ampel).

Tabelle 10: Prozent des Textens nach Komplexitätseinstufung nach Fastenmeier $(N=41)$.

\begin{tabular}{|l|c|c|c|c|c|}
\hline Komplexität & N & $\boldsymbol{M}$ & SD & Min & Max \\
\hline Hoch & 8 & 22,3 & 19,0 & 0,0 & 63,4 \\
\hline Mittel & 9 & 39,0 & 25,7 & 0,0 & 92,7 \\
\hline Gering & 9 & 61,8 & 25,0 & 24,4 & 95,1 \\
\hline Situationen ohne Komplexitätseinstufung & 17 & 44,6 & 29,6 & 2,4 & 97,6 \\
\hline
\end{tabular}

Bezogen auf den Straßentyp zeigte sich, dass für jeden Straßentyp anscheinend ähnlich schwierige Situationen ausgewählt wurden. Bei jedem Straßentyp würden zwischen $40 \%$ und $50 \%$ der Teilnehmer eine Textnachricht schreiben (siehe Tabelle 11). Die Spannweite reichte für alle Straßentypen von (nahezu) $0 \%$ bis mindestens knapp $80 \%$ der Teilnehmer.

Tabelle 11: Prozent des Textens nach Straßentyp $(N=41)$.

\begin{tabular}{|l|c|c|c|c|c|}
\hline Komplexität & N & $\boldsymbol{M}$ & SD & Min & Max \\
\hline Autobahn & 14 & 43,0 & 27,9 & 0,0 & 92,7 \\
\hline Landstraße & 8 & 47,3 & 27,3 & 0,0 & 78,0 \\
\hline Innenstadt & 21 & 41,1 & 30,1 & 2,4 & 97,6 \\
\hline
\end{tabular}

In der Folge wurde überprüft, inwieweit die in den verschiedenen Situationen abgegebene Bereitschaft zu Texten mit der wahrgenommenen Erhöhung des Risikos eines Unfalls durch dieses Verhalten zusammenhängt. Im Mittel wurde das Risiko um $48,2 \%(S D=18,1 \%)$ erhöht eingeschätzt, wenn eine Textnachricht geschrieben würde. In Abbildung 8 zeigt sich, dass in Situationen in denen die meisten Teilnehmer eine Textnachricht schreiben würden, die Erhöhung des Risikos als gering eingeschätzt wurde, während in den Situationen, in denen nur eine geringe Bereitschaft zum Texten bestand, auch das Unfallrisiko als teilweise deutlich erhöht bewertet wurde. Entsprechend ergab sich eine signifikant negative Korrelation zwischen der Anzahl der Teilnehmer die in der jeweiligen Situation eine Textnachricht verfasst hätten und der Risikoerhöhung $(r=-, 884 ; p<, 001)$. 


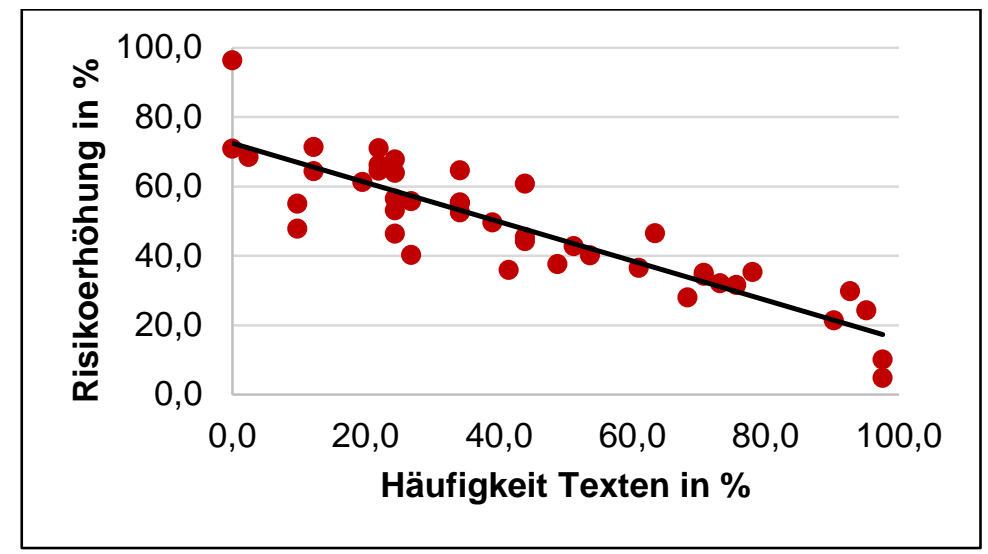

Abbildung 8: Zusammenhang zwischen Risikoerhöhung (in \%) und der Häufigkeit eine Textnachricht (in \%) zu schreiben $(N=41)$.

\subsubsection{Qualitative Aussagen zum Einfluss von Kontextvariablen auf das Verfassen von Textnachrichten}

Als Grund für die Bereitschaft eine Textnachricht zu schreiben wurde auffällig häufig angegeben, dass in den zugehörigen Situationen keine Gefahr durch andere Verkehrsteilnehmer bestünde (siehe Abbildung 9). Ein Teilnehmer drückte es folgendermaßen aus: „erscheint mir sicher, weil ich niemanden gefährde außer mich“. Außerdem wurden als weitere wichtige Gründe viel Platz und Abstand zu anderen Verkehrsteilnehmern genannt, gute Sicht- und Lichtverhältnisse, sowie auch eine moderate bzw. geringe Geschwindigkeit. Ein Teilnehmer sagte dazu: „man ist sowieso schon am Verzögern“. Andere empfanden auch ihr eigenes Fahrverhalten in der Situation als sicher, z.B. „wenn was ist, kann ich abbremsen“.

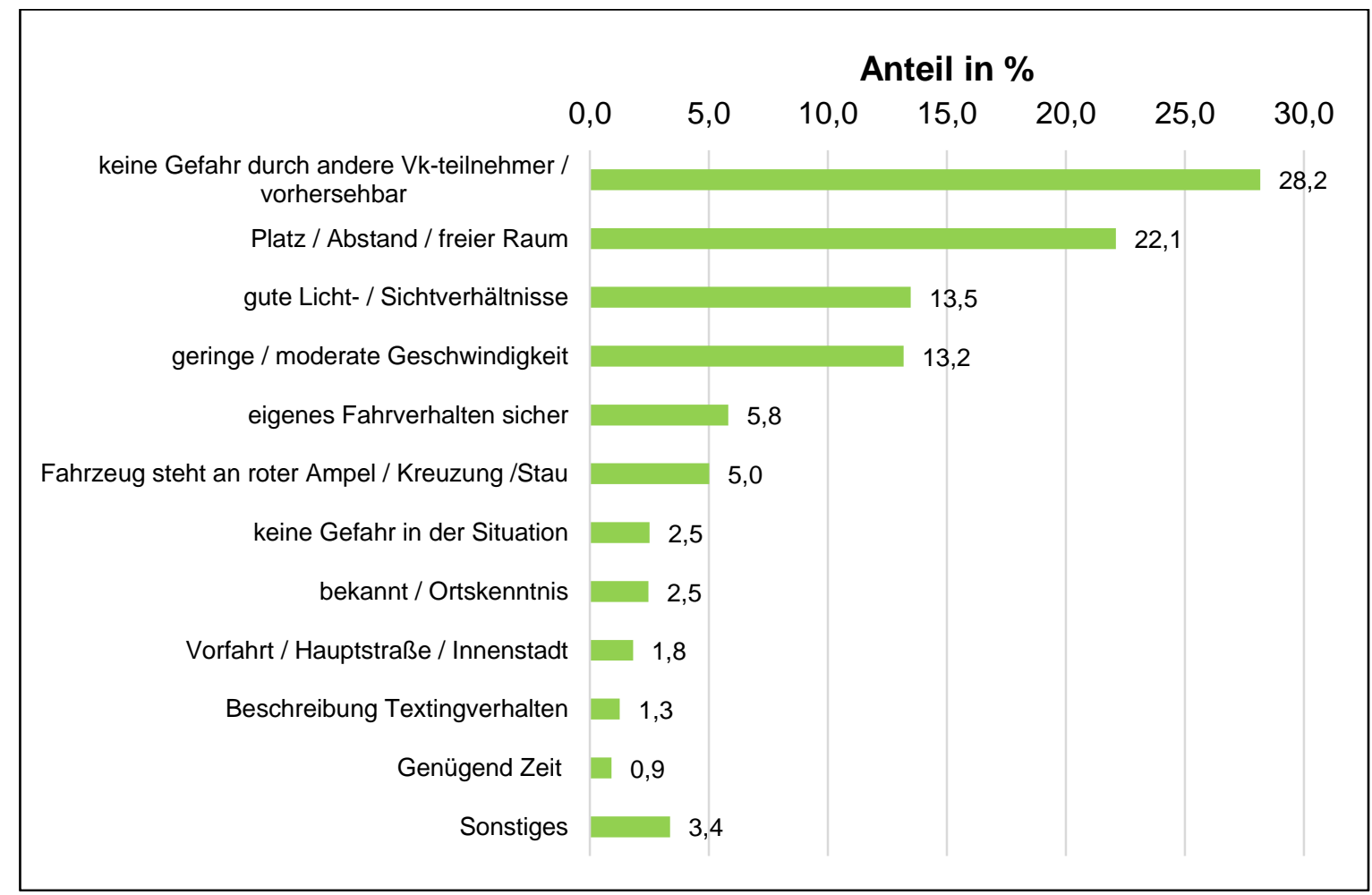

Abbildung 9: Nennungen (in \%) für Argumente warum Teilnehmer eine Textnachricht verfassen würden $(N=41)$. 
Als Gründe gegen das Verfassen einer Textnachricht in den gezeigten Situationen wurde mit großer Mehrheit genannt, dass eine Gefahr durch andere Verkehrsteilnehmer bestünde bzw. sich andere unberechenbar verhielten (siehe Abbildung 10). Ein Teilnehmer sagte, Vorausfahrender „bremst in Kurven sehr runter, wo ich dann doch schneller fahr". Als weiteren Ausschlussgrund wurden eine enge Fahrbahn oder anderweitige Verengungen der Straße genannt. Außerdem spielte, übereinstimmend mit der Definition von Fastenmeier (1995), auch die Komplexität der eigenen Fahraufgabe eine Rolle. Ebenso wurde allgemein die fehlende Übersichtlichkeit der Verkehrssituation als Hinderungsgrund benannt. Die Teilnehmer schienen auch die Vorfahrtsregelungen an Knotenpunkten in ihre Entscheidung einzubeziehen, z.B. „Vorfahrtsstraße, bin zwar drauf, aber könnte ja trotzdem jemand kommen“. Ferner wurde in einigen Fällen in Betracht gezogen, dass möglicherweise ein Blitzer oder die Polizei an der Stelle stehen könnte oder dass sich später eine bessere Möglichkeit zum Schreiben der Nachricht ergibt.

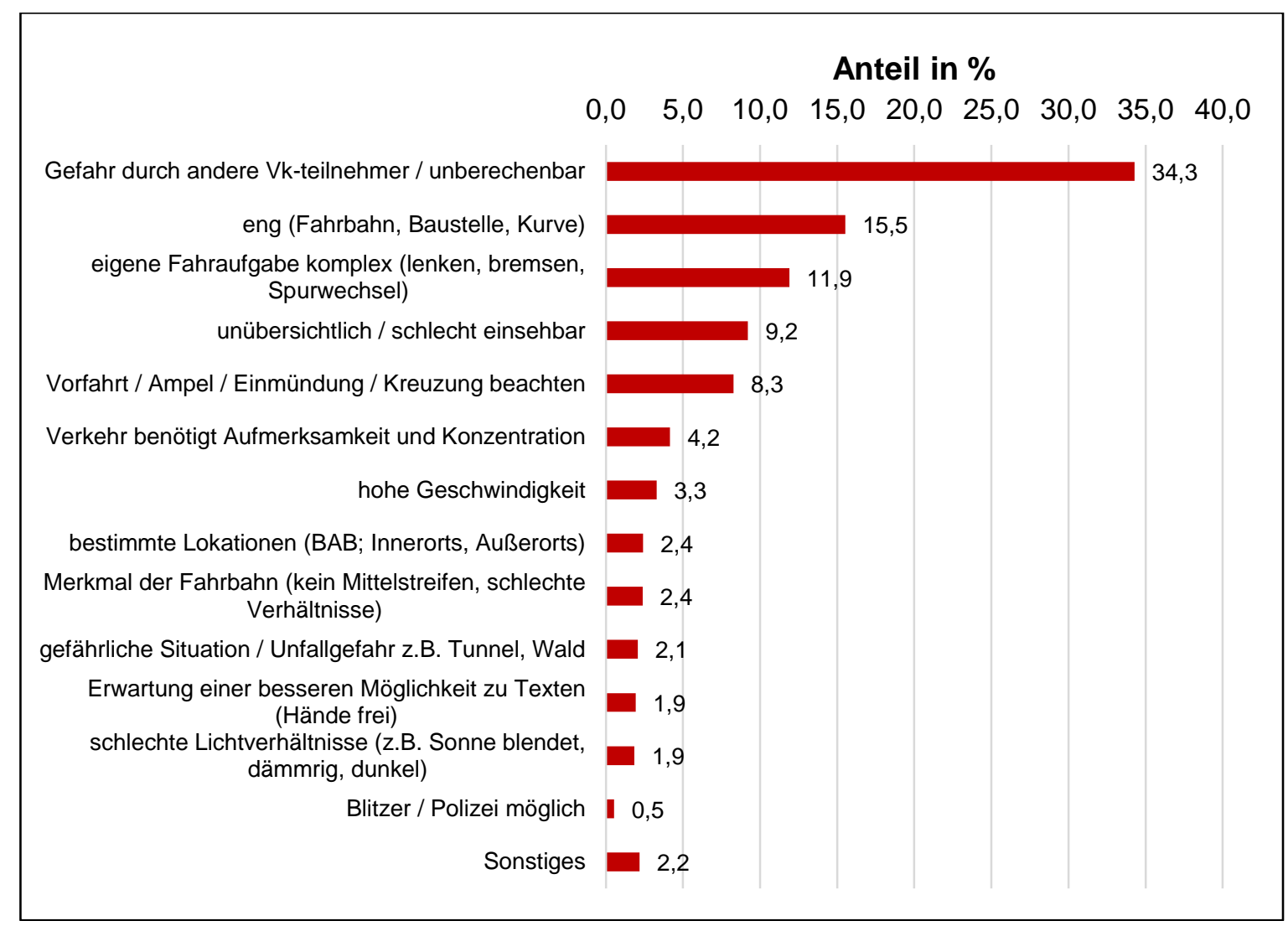

Abbildung 10: Nennungen (in \%) für Argumente warum Teilnehmer keine Textnachricht verfassen würden $(N=41)$. 
Auf die Frage „Was müsste anders sein, damit sie keine Textnachricht in der Situation verfassen?" (wenn zuvor eine Bereitschaft zum Texten signalisiert wurde) nannte die Hälfte der Teilnehmer, dass die deutlichere Präsenz von bzw. notwendige Interaktion mit anderen Verkehrsteilnehmern ein solcher Faktor wäre, z.B. „Fußgänger (Kinder, die spielen), Radler" (siehe Abbildung 11). Dabei wurden vornehmlich nicht-motorisierte Verkehrsteilnehmer genannt. Des Weiteren würden anscheinend schlechte Sicht und Wetterbedingungen, z.B. „Dunkelheit oder Regen“, ebenso wie eigene Fahrmanöver (lenken, bremsen, abbiegen) dazu führen, dass die Teilnehmer vom Schreiben der Textnachricht absehen würden.

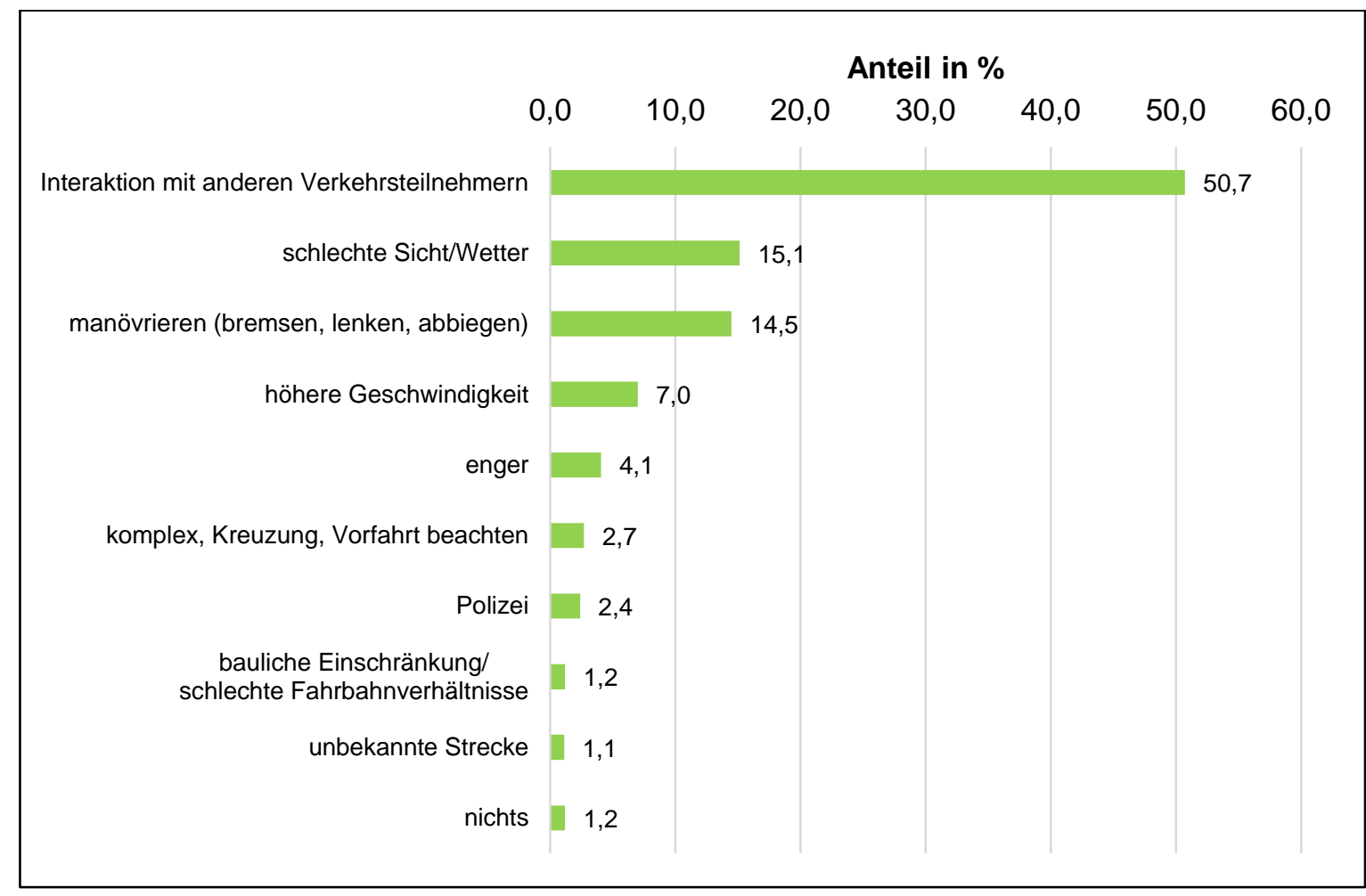

Abbildung 11: Nennungen (in \%) für Argumente was anders sein müsste, damit die Teilnehmer nicht mit dem Verfassen einer Textnachricht beginnen $(N=41)$. 
Auf die Frage, welches eine Merkmal anders sein müsste, damit sie in der Situation beginnen würden eine Textnachricht zu verfassen (wenn zuvor keine Bereitschaft bestand), antworteten die Teilnehmer sehr unterschiedlich. Dabei stach keine Begründung besonders hervor. Die meisten antworteten, dass sie beginnen würden, wenn keine anderen Verkehrsteilnehmer zugegen wären, oder generell wenig Verkehr sei, z.B. „nicht so viele Störfaktoren rechts und links bzw. geringe Verkehrsdichte“ (siehe Abbildung 12) Knapp 15\% der genannten Begründungen zielten auf eine breite bzw. gerade Strecke als Voraussetzung ab. Erwähnenswert ist auch, dass in einem erheblichen Teil der Fälle angegeben wurde, dass die Teilnehmer in der entsprechenden Situation nichts dazu hätte bewegen können eine Textnachricht zu schreiben.

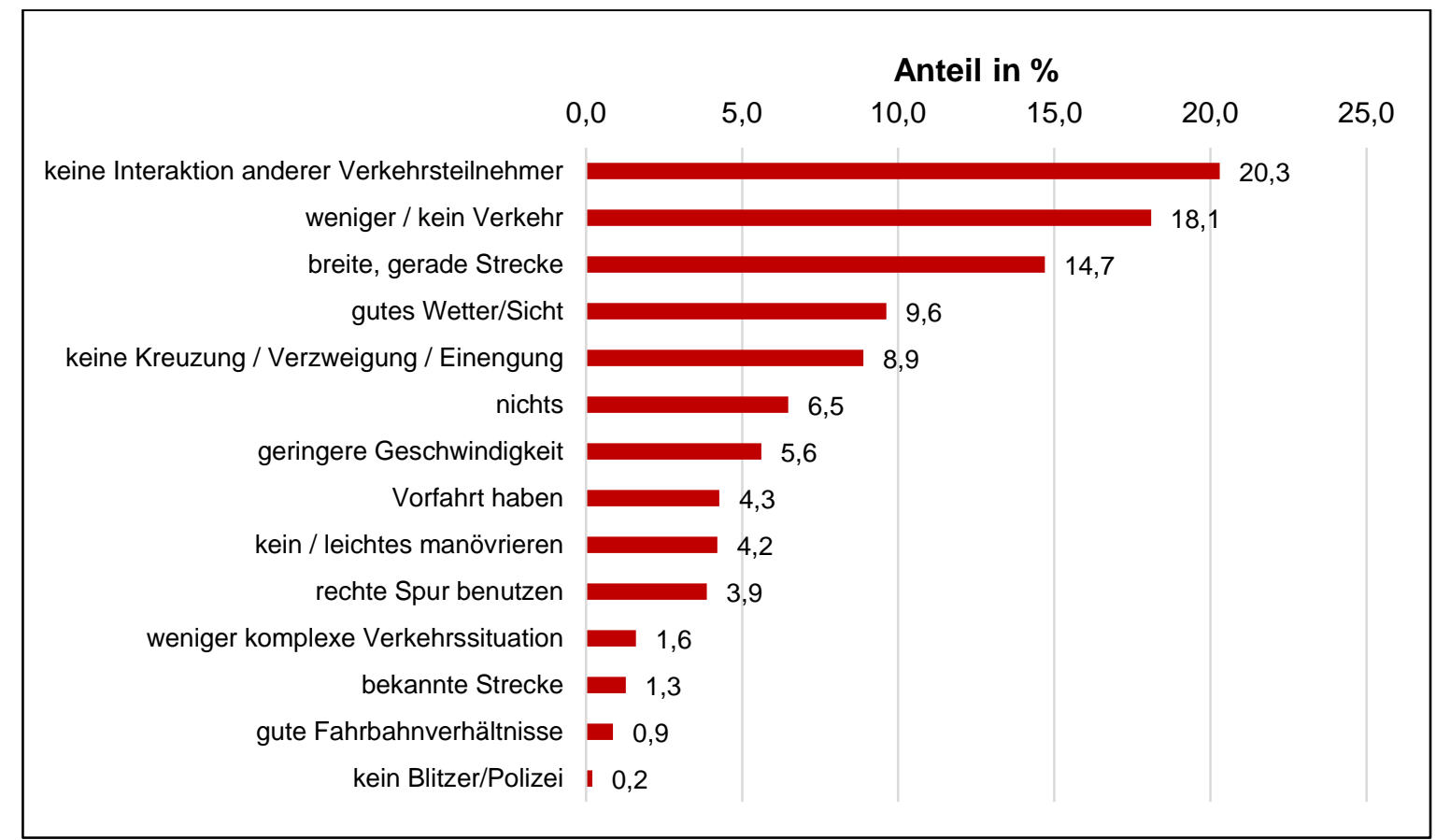

Abbildung 12: Nennungen (in \%) für Argumente was anders sein müsste, damit die Teilnehmer mit dem Verfassen einer Textnachricht beginnen $(N=41)$. 


\subsubsection{Einfluss von überdauernden (Persönlichkeits-) eigenschaften auf das Verfassen von Textnachrichten während des Fahrens}

\section{Einstellung zum Schreiben von Textnachrichten während des Fahrens}

Die Einstellung zum Schreiben von Textnachrichten während des Fahrens wurde mit einer Skala bestehend aus fünf Items erfasst (z.B. Textnachrichten während des Fahrens zu verfassen, ist nicht ablenkend). Die Teilnehmer sollten ihre Zustimmung auf sieben Stufen (1 - starke Ablehnung bis 7 - starke Zustimmung) angeben. In Abbildung 13 lässt sich erkennen, dass die Teilnehmer dem Schreiben von Textnachrichten während des Fahrens durchaus positiv gegenüber eingestellt waren $(M=4,5 ; S D=0,6)$. Das ist nicht überraschend, da die Stichprobe bewusst aus Teilnehmern zusammengestellt wurde, die dieses Verhalten grundsätzlich zeigen. Im vorliegenden Fall zeigte sich für die Teilnehmer allerdings kein Zusammenhang zwischen der Einstellung und der in der Untersuchung identifizierten Anzahl an Situationen, in denen die Teilnehmer eine Textnachricht schreiben würden $(r=, 017 ; p=, 917)$. Dies spricht für einen starken Einfluss situativer Merkmale auf die Bereitschaft bzw. Ablehnung eine Textnachricht zu verfassen. Dies ist weitgehend unabhängig von der grundsätzlich positiven Einstellung dem Texten beim Fahren gegenüber.

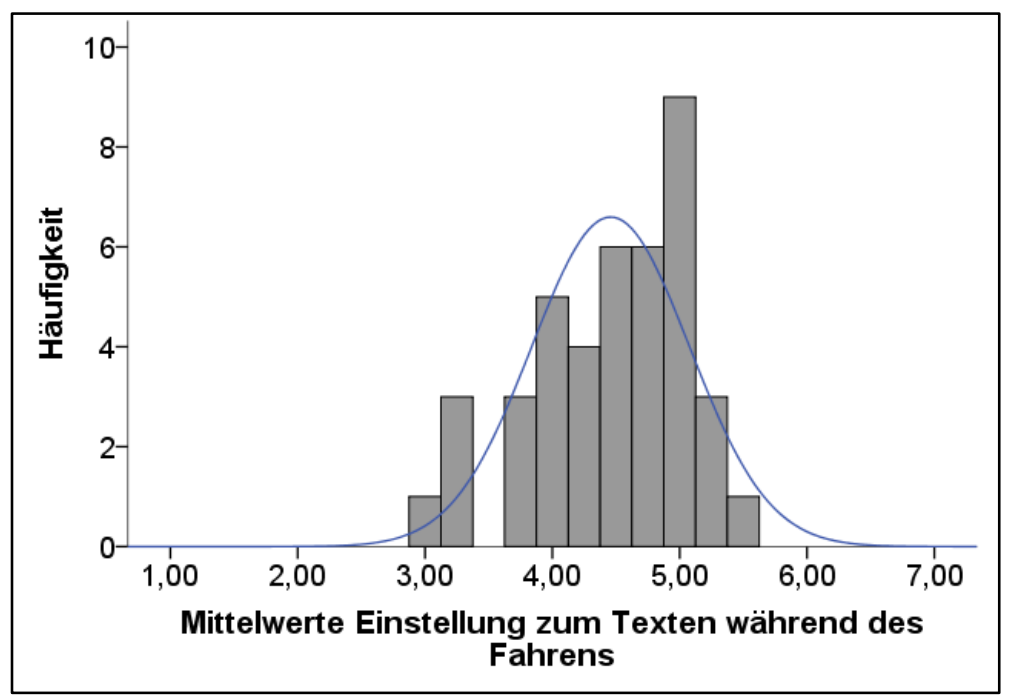

Abbildung 13: Häufigkeit der Mittelwerte der Teilnehmer auf der Skala zur Einstellung gegenüber dem Schreiben von Textnachrichten während des Fahrens (Skala 1-7; $N=41$ ). 
Auf einer 7-stufigen Zustimmungsskala wurde erfasst, wie stark die Teilnehmer eine Textnachricht aus Gewohnheit schreiben (z.B. Textnachrichten schreiben ist etwas, dass ich tue ohne darüber nachzudenken.). In Abbildung 14 ist die Zustimmung über die Teilnehmer hinweg dargestellt, höhere Werte auf $x$-Achse sprechen für starkes gewohnheitsmäßiges Schreiben von Textnachrichten. Die meisten Teilnehmer berichteten eine mittlere Zustimmung $(M=3,5 ; S D=1,3)$. Gleichzeitig bestand auch hier nur ein geringer Zusammenhang zwischen der berichteten Gewohnheit und der in der Untersuchung identifizierten Anzahl an Situationen, in denen eine Bereitschaft zum Schreiben einer Textnachricht geäußert wurde $(r=, 157 ; p=, 327)$, was als weiterer Beleg für den starken Einfluss situativer Merkmale zu werten ist.

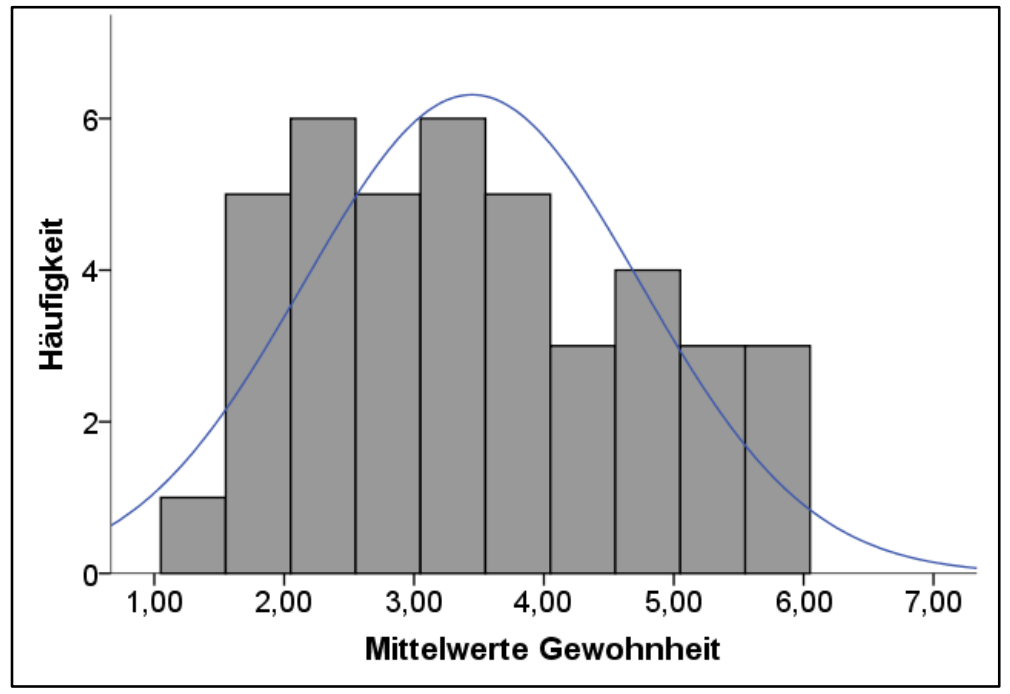

Abbildung 14: Häufigkeit der Mittelwerte der Teilnehmer auf der Skala zum gewohnheitsmäßigen Schreiben von Textnachrichten (Skala 1-7, $N=41$ ).

\section{Einstellung, Normen und Verhaltenskontrolle}

Auch für die verschiedenen Teilkomponenten der Theorie des geplanten Verhaltens wurden Antworten mit Hilfe einer 7-stufigen Skala erfasst. Dabei handelte es sich um die Komponenten Einstellung, Subjektive Verhaltenskontrolle und subjektive Norm, welche sich in wahrgenommene Normen, moralische Normen und Gruppennormen gliedert. Für die Skala zur Einstellung kam ein sogenanntes semantisches Differential zur Anwendung, auf dem die Teilnehmer ihre Einschätzung zwischen zwei benannten Polen (z.B. "gut" und „schlecht") einordnen mussten. Die Daten zeigen, dass die Teilnehmer gegenüber dem Schreiben von Textnachrichten während des Fahrens im Durchschnitt neutral eingestellt waren (siehe Abbildung 15). Es konnte dabei ein mittlerer statistisch signifikanter Zusammenhang zwischen dieser Einstellung und der Anzahl der Textnachrichten, die die Teilnehmer in den 43 ausgewählten Situationen schreiben würden, gefunden werden (siehe Tabelle 12). Das bedeutet, dass die Teilnehmer die dem Verhalten gegenüber positiver eingestellt waren auch tendenziell mehr Textnachrichten verfasst hätten. Der Mittelwert für die Kontrolle darüber Textnachrichten während des Fahrens zu schreiben lag über dem Skalenmittel. Das heißt, die Teilnehmer wurden gefragt, ob sie der Ansicht waren, die Kontrolle über das Verhalten zu haben. Die subjektive Norm gliedert sich nochmals in die wahrgenommene Norm, moralische Norm und Gruppennorm, welche auch getrennt ausgewertet wurden. Für die Skala zur Gruppennorm zeigt sich, dass die Teilnehmer offenbar tendenziell eher der Meinung waren, dass auch Freunde oder Verwandte das Schreiben von Textnachrichten 
während des Fahrens gutheißen. Die Werte zu wahrgenommenen Normen und moralischen Normen lagen im mittleren Bereich. Gleichzeitig wurde ein mittlerer signifikant negativer Zusammenhang zwischen der eigenen moralischen Norm und der Anzahl der Situationen, in denen die Teilnehmer ihre Bereitschaft zu texten signalisierten, gefunden. Das heißt, dass die Teilnehmer, die in weniger Situationen Textnachrichten verfasst hätten, dieses Verhalten allgemein moralisch fragwürdiger fanden.

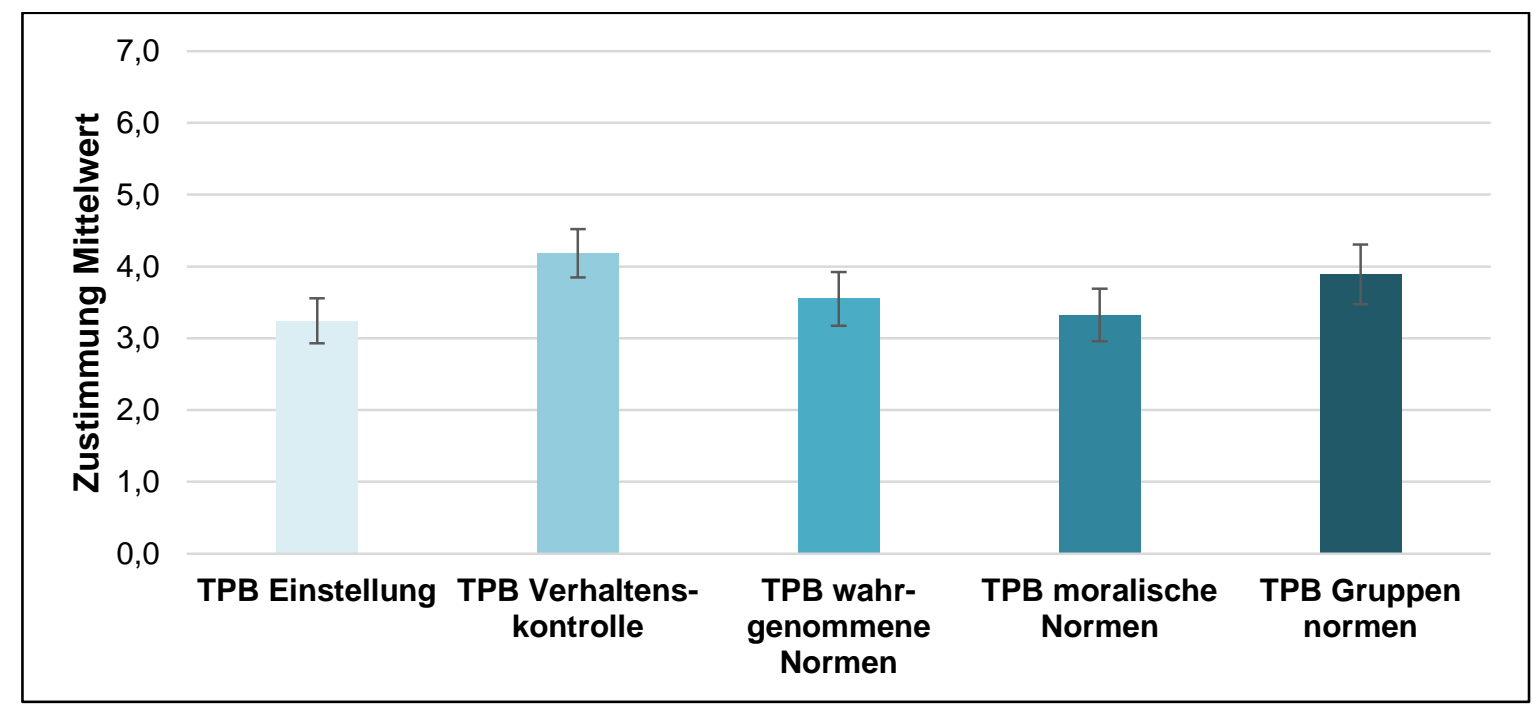

Abbildung 15: Mittelwerte der Zustimmung für die Skalen der Theorie des geplanten Verhaltens bezogen auf das Schreiben von Textnachrichten während des Fahrens $(N=41)$.

Tabelle 12: Korrelationen der Skalen der Theorie des geplanten Verhaltens mit Anzahl der Situationen in denen Textnachricht verfasst worden wären $(N=41)$.

\begin{tabular}{|l|c|c|}
\hline Skala & $\boldsymbol{r}$ & $\boldsymbol{p}$ \\
\hline TPB Einstellung &. $\mathbf{3 1 8}$ &. $\mathbf{0 4 2}$ \\
\hline TPB Verhaltenskontrolle & .267 & .091 \\
\hline TPB Wahrgenommene Normen & -.197 & .217 \\
\hline TPB Moralische Normen & -.314 &. $\mathbf{0 4 5}$ \\
\hline TPB Gruppennormen & -.039 & .807 \\
\hline
\end{tabular}

\section{Fahrstil}

Zusätzlich wurde der Fahrstil der Teilnehmer erfasst. Hierbei wurde in vorsichtigen und routinierten Fahrstil unterschieden (Popken, 2009). Insgesamt bewerteten sich die Teilnehmer auf einer 7-stufigen Skala als eher routinierte Fahrer $(M=5,0, S D=0,4)$ und als durchschnittlich vorsichtig $(M=3,1, S D=0,7)$. Es konnte ein mittlerer Zusammenhang zwischen vorsichtigem Fahrstil und der Anzahl der Situationen, in denen eine Textnachricht verfasst worden wäre, identifiziert werden $(r=-, 257 ; p=, 105)$. Dagegen bestand nur ein kleiner Zusammenhang zwischen routiniertem Fahrstil und der Anzahl der Situationen, in denen Textnachricht verfasst worden wäre $(r=, 110 ; p=, 492)$. 


\subsection{Zusammenfassung}

In der videobasierten, strukturierten Interviewstudie zeigte sich wie erwartet, dass die jeweils vorherrschende Verkehrssituation einen entscheidenden Einfluss auf die Bereitschaft eine Textnachricht zu verfassen ausübt. So wurden Situationen identifiziert, in denen nahezu jeder der Teilnehmer eine Textnachricht während des Fahrens schreiben würde. Genauso gab es aber auch Situationen, in denen kein oder kaum ein Teilnehmer eine Textnachricht verfassen würde. Auch wenn den Teilnehmern eine grundsätzlich hohe Bereitschaft während des Fahrens zu texten zugeschrieben werden konnte, und sie diesem Verhalten auch generell eher positiv gegenüberstanden, so schienen sie letztlich doch im konkreten Fall die Entscheidung für oder gegen das Texting anhand von situativen Merkmalen zu treffen. Für die erhobenen überdauernden Personenmerkmale (Einstellungen gegenüber dem Verfassen von Textnachrichten, Fahrstil usw.) hingegen konnten nur verhältnismäßig schwache Zusammenhänge zur Anzahl der Situationen, in denen die Teilnehmer eine Textnachricht schreiben würden, gefunden werden.

Das Ziel der videobasierten, strukturierten Interviewstudie bestand darin Kontexte zu identifizieren, bei denen Autofahrer bereit oder gerade nicht bereit sind während des Fahrens eine Textnachricht zu schreiben. Durch die Analyse der Interviewdaten konnten einige übergeordnete Merkmale identifiziert werden. Als wichtiger Aspekt wurde die Anwesenheit von anderen Verkehrsteilnehmern identifiziert. Die Gegenwart von Fußgängern oder Radfahrern, oder auch die hohe Verkehrsdichte auf der Fahrbahn wurden wiederholt als Hinderungsgründe genannt. Auch hätte das „Entfernen“ dieser Verkehrsteilnehmer aus der jeweiligen Situation nach Angabe der Untersuchungsteilnehmer in vielen Fällen die Bereitschaft zum Schreiben einer Textnachricht erhöht. Von manchen Teilnehmern wurde dieser starke Einfluss damit begründet, dass sie dann niemand anderen gefährden außer sich selbst. Außerdem sind andere Verkehrsteilnehmer auch dadurch eine Gefahrenquelle, weil sie sich nicht immer in der erwarteten Weise verhalten.

Die Komplexität der Fahraufgabe scheint auch unabhängig von der Beteiligung anderer Verkehrsteilnehmer als übergeordnetes Merkmal bei den Gründen für und gegen das Schreiben von Textnachrichten eine große Rolle zu spielen. In Situationen, die laut der Klassifikation von Fastenmeier (1995) als hoch komplex gewertet wurden, wurde das Verfassen von Textnachrichten eher unterlassen. Auch die Kontextfaktoren die dazu beitrugen, dass sich die Teilnehmer entschlossen keine Nachricht zu schreiben, wie etwa enge Straßen, zu beachtende Vorfahrt oder eine hohe Komplexität der eigenen Fahraufgabe unterstreichen die Wichtigkeit der Komplexität als Einflussfaktor auf die Bereitschaft Nachrichten zu schreiben. Auch wurden schlechte Sicht- und Wetterbedingungen genannt, welche bei Fastenmeier (1995) per se als komplexitätssteigernd angesehen werden. Im Umkehrschluss bedeutet dies, dass Autofahrer bei Abwesenheit dieser Faktoren durchaus bereit sein sollten eine Textnachricht zu schreiben, was auch durch die Ergebnisse des Interviews bestätigt werden konnte.

Zentral ist den meisten vorgebrachten Argumenten, dass sie implizit oder auch explizit auf die Vorhersehbarkeit der Verkehrssituation abzielen. Erscheint die Situation vorhersehbar, so erhöht sich auch die Bereitschaft beim Fahren einer Textnachricht zu verfassen. Unmittelbar mit dieser Vorhersehbarkeit verknüpft ist die Frage, inwieweit der Fahrer in der jeweiligen Situation unmittelbar reaktionsbereit sein muss (weil die Vorhersehbarkeit gering ist), oder ggf. etwas mehr Zeit zum Reagieren zur Verfügung steht. Letztlich scheinen sich die Fahrer ihrer sensorischen und kognitiven 
Einschränkungen durch das Schreiben einer Textnachricht durchaus bewusst zu sein, und wählen für die Bearbeitung der Aufgabe entsprechend Situationen, in denen innen die vermeintliche Vorhersehbarkeit einen gewissen zeitlichen Puffer verschafft. Allerdings ist fraglich, inwieweit sich diese subjektive Einschätzung der Situation immer mit der objektiven Realität deckt. Diese Problematik wird nicht zuletzt auch an den Situationen erkennbar, bei denen es innerhalb der Untersuchung keinen Konsens bzgl. der Eignung zum Schreiben einer Textnachricht gab, und bei denen von verschiedenen Teilnehmern teilweise einander entgegengesetzte Argumente geliefert wurden. 


\section{Fahrsimulatorstudie}

Wie die Literaturrecherche (Kapitel 2), die Re-Analyse der SHRP2-Daten (Kapitel 3) sowie die Interviewstudie (Kapitel 4) deutlich gemacht haben, sind sich Fahrer der von der Bearbeitung einer Nebentätigkeit (z.B. Texten) ausgehenden Beanspruchung durchaus bewusst und passen entsprechend ihr Fahrverhalten oder auch die Bearbeitung der Nebentätigkeit situationsabhängig an. So existieren offensichtlich bestimmte kontextuelle Bedingungen die dazu führen, dass die Fahrer das Bearbeiten von Textnachrichten unterlassen, ebenso wie es Kontexte gibt, in denen Fahrer eine erhöhte Bereitschaft zum Lesen oder Schreiben von Textnachrichten zeigen. Inwieweit die Kompensation allerdings ausreichend, bzw. inwieweit die Auswahl von für das Texten „geeigneten“ Kontexten tatsächlich angemessen ist, ist unklar. Speziell stellt sich dabei die Frage, wie Fahrer mit potentiell kritischen Situationen umgehen, die sich in vermeintlich einfachen, scheinbar sicheren Fahrkontexten entwickeln.

Um dies untersuchen zu können wurden in der Fahrsimulatorstudie Kontexte umgesetzt, bei denen eine hohe Bereitschaft zum Verfassen von Textnachrichten bei den Fahrern zu erwarten war. Die Fahrer sollten während des Fahrens einer von vier Nebentätigkeiten nachgehen:

1. Schreiben von Textnachrichten am Mobiltelefon,

2. Lesen von Textnachrichten am Mobiltelefon,

3. Spracheingabe von Textnachrichten durch ein sprachbasiertes System, oder

4. Vorlesen lassen von Textnachrichten durch ein sprachbasiertes System.

Dabei hatten die Fahrer jederzeit die Möglichkeit eine Nachricht nicht zu bearbeiten oder zu unterbrechen, so dass eine Anpassung des Verhaltens an die Situation möglich war. Diese Aufgaben wurden ausgewählt, da sie die beiden zentralen Funktionen von Messenger-Diensten und vergleichbaren Apps (Nachricht lesen und Nachricht schreiben) abbilden. Durch die freie Entscheidung für oder gegen die Nebenaufgabenbearbeitung sollten die Ergebnisse eine höhere Validität aufweisen als die Befunde vergleichbarer Fahrsimulatorstudien, in denen diese Freiheit nicht bestand.

Des Weiteren sollte vor dem vor dem Hintergrund aktueller rechtlicher und technischer Entwicklungen der Einfluss einer sprachbasierten Ein- und Ausgabe auf das Fahrverhalten untersucht werden. Eine Kontrollgruppe durchfuhr die simulierte Strecke ohne Nebentätigkeit.

Innerhalb der Strecke wurden einfache, unkritische Abschnitte zur Betrachtung der generellen Fahrperformanz bei der Ausführung von Nebentätigkeiten umgesetzt. Des Weiteren wurden Situationen umgesetzt, die zunächst vermeintlich einfach waren, sich in der Folge aber zu einer kritischen Situation entwickelten, um die Fahrperformanz in Grenzsituationen zu untersuchen. Einige dieser Situationen enthielten zudem Hinweise, im unmittelbaren Vorfeld auf die kritische Situation. Dies sollte ermöglichen genauer zu betrachten, inwieweit sich die verschiedenen Nebenaufgaben auf die Fahrleistung auswirken. Dabei sollten sowohl die reine Reaktionsfähigkeit, als auch die Verarbeitung von situationsrelevanten Informationen Beachtung finden. Zweck dieser Variationen war es, ein möglichst ganzheitliches Bild der Folgen verschiedener Formen und Teilaspekte des Textings auf die Fahrperformanz zu zeichnen. 


\subsection{Methode}

\subsubsection{Stichprobe}

Die Rekrutierung der Teilnehmer erfolgte über einen Aufruf in regionalen Medien, über die Homepage der Technischen Universität Chemnitz, sowie eine Auswahl aus der Probandendatenbank der Professur Allgemeine und Arbeitspsychologie. Außerdem wurden die Teilnehmer der Interviewstudie angesprochen. Die Bewerber füllten online einen Fragebogen zur Kontaktaufnahme aus. Der Fragebogen enthielt u.a. Angaben zu soziodemografischen Variablen (Alter, Geschlecht), zum Fahrzeug und zu mobiltelefonbezogenen Aspekten (Art, Nutzungshäufigkeit beim Fahren).

Insgesamt bewarben sich 705 Personen um eine Teilnahme an der Fahrsimulatorstudie, deren Zahl in einem Auswahlprozess sukzessive reduziert wurde. Es wurden Bewerber bevorzugt, die ihr Mobiltelefon möglichst häufig während des Fahrens zum Verfassen von Textnachrichten nutzten, sowie hinreichend erfahrene Autofahrer sind.

Insgesamt nahmen 98 Probanden an der Studie teil, wobei 16 Datensätze nicht verwertbar waren, da zehn Probanden wegen Simulatorkrankheit und sechs Teilnehmer auf Grund von technischen Problemen des Fahrsimulators oder Kameras vor dem Ende der Fahrten abbrechen mussten. Entsprechend flossen die Datensätze von 82 Teilnehmern (41 Männer, 41 Frauen), mit einem Durchschnittsalter von 30,3 Jahren (18 bis 63 Jahre) in die Analyse ein. Die Verteilung der Teilnehmer auf die verschiedenen Versuchsbedingungen ist in Tabelle 13 dargestellt.

Tabelle 13: Verteilung Teilnehmer auf Versuchsbedingungen $(N=82)$.

\begin{tabular}{|l|l|l|l|l|l|l|}
\hline & \multicolumn{2}{|c|}{ Frauen } & \multicolumn{2}{c|}{ Männer } & \multicolumn{2}{c|}{ Gesamt } \\
\hline Bedingung & $N$ & $M_{\text {Alter }}(S D)$ & $N$ & $M_{\text {Alter }}(S D)$ & $N$ & $M_{\text {Alter }}(S D)$ \\
\hline Baseline / Baseline & 9 & $35,0(10,9)$ & 8 & $32,0(13,1)$ & 17 & $33,6(11,7)$ \\
\hline Lesen / Schreiben & 9 & $24,7(5,5)$ & 8 & $29,9(8,2)$ & $\mathbf{1 7}$ & $27,1(7,2)$ \\
\hline Schreiben / Lesen & 7 & $26,1(3,7)$ & 9 & $28,8(6,5)$ & $\mathbf{1 6}$ & $27,6(5,4)$ \\
\hline $\begin{array}{l}\text { Vorlesen / } \\
\text { Spracheingabe }\end{array}$ & 8 & $26,5(3,6)$ & 8 & $27,5(4,0)$ & $\mathbf{1 6}$ & $27,0(3,7)$ \\
\hline $\begin{array}{l}\text { Spracheingabe / } \\
\text { Vorlesen }\end{array}$ & 8 & $39,5(13,3)$ & 8 & $32,4(10,0)$ & $\mathbf{1 6}$ & $35,9(11,9)$ \\
\hline
\end{tabular}

Die Teilnehmer besaßen den Führerschein der Klasse B (Pkw) im Durchschnitt seit 12,5 Jahren (1 bis 44 Jahre). Die durchschnittliche jährliche Fahrleistung betrug $29.773 \mathrm{~km}$ (2.500 bis $350.000 \mathrm{~km}$ ). Die Mehrheit der Teilnehmer fuhr überwiegend einen Pkw $(n=75)$, nur drei fuhren überwiegend Kleintransporter, vier einen Lkw. Außerdem nutzten 81 Teilnehmer ein Smartphone, lediglich ein Teilnehmer nutzte ein konventionelles Mobiltelefon (Verteilung auf Hersteller siehe Tabelle 14tabelle 9). 68,7\% der Teilnehmer nutzten das Betriebssystem Android, 25,3\% iOS, der Rest nutzte andere Betriebssysteme (2,4\%) bzw. machte keine Angabe (3,6\%). Zum Verfassen von Nachrichten nutzten drei Viertel der Teilnehmer gewöhnlich WhatsApp $(73,0 \%)$, einige wenige SMS (10,8\%) und Facebook (11,9\%), sowie sonstige Dienste $(4,3 \%)$. 
Tabelle 14: Überblick über die genutzten Mobiltelefone $\left(n=83^{14}\right)$.

\begin{tabular}{|l|c|c|}
\hline Art Mobiltelefon & Häufigkeit & Prozent \\
\hline Samsung & 33 & 39,8 \\
\hline Apple & 21 & 25,3 \\
\hline Sony & 11 & 13,3 \\
\hline Huawei & 5 & 6,0 \\
\hline HTC & 4 & 4,8 \\
\hline LG & 3 & 3,6 \\
\hline Sonstiges & 6 & 7,2 \\
\hline
\end{tabular}

\subsubsection{Material}

\section{Fahrsimulator}

Für die Untersuchung wurde ein statischer Fahrsimulator mit Mock-Up der Vorderfront eines Fahrzeugs bis einschließlich B-Säule und Blickwinkel von $180^{\circ}$ verwendet (siehe Abbildung 16). Das Fahrzeug verfügte über aktive Pedalerie und Lenkung. Gangwechsel erfolgten in Form eines Automatikgetriebes. Als Simulationsumgebung wurde SILAB 5.0 verwendet. Die Software ermöglichte die Aufzeichnung aller gängigen Fahrparameter, so z.B. Geschwindigkeit, Lenkwinkel, Bremsdruck oder Spurposition.

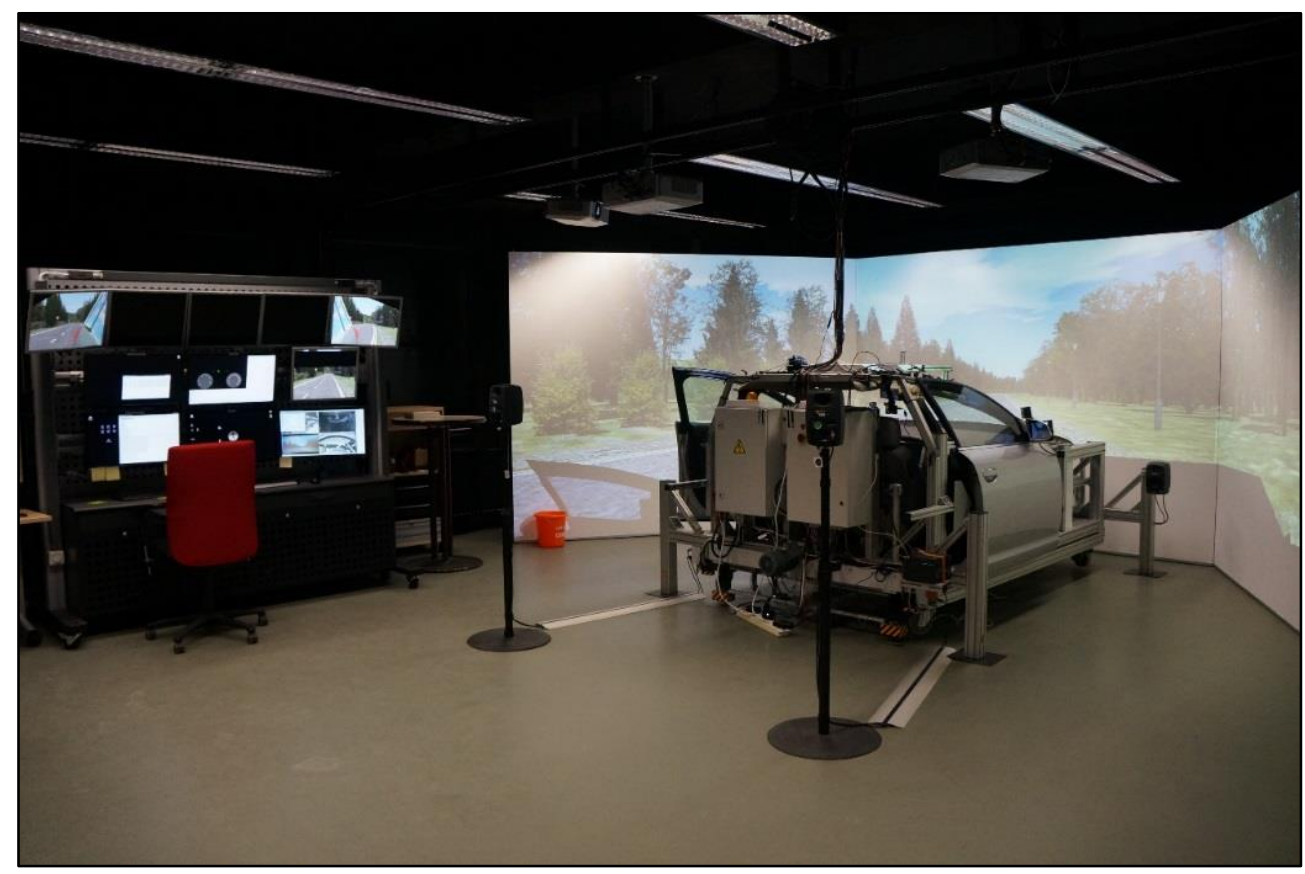

Abbildung 16: Überblick über Fahrsimulator inklusive Versuchsleiterarbeitsplatz.

Zur besseren Beschreibung des Fahrerverhaltens wurde zusätzlich ein aus vier Kameras bestehendes integriertes Kamerasystem verwendet. Je eine Kamera filmte das Gesicht des Fahrers, den Oberkörper, die Voraussicht auf Strecke und den Fußraum (siehe 
Abbildung 17). Zusätzlich wurde eine Innenraumkamera (GoPro Hero4 Black) und eine Szenenkamera (Sony Handycam, HDR-CX 115E siehe Abbildung 18, links) genutzt. Die Innenraumkamera filmte den Blick vom „Rücksitz“ auf den Fahrzeuginnenraum, um die Bedienung des Smartphones besser erfassen zu können. Die Szenenkamera filmte die Strecke hochauflösend vom Dach des Mock-Ups (siehe Abbildung 18, rechts).

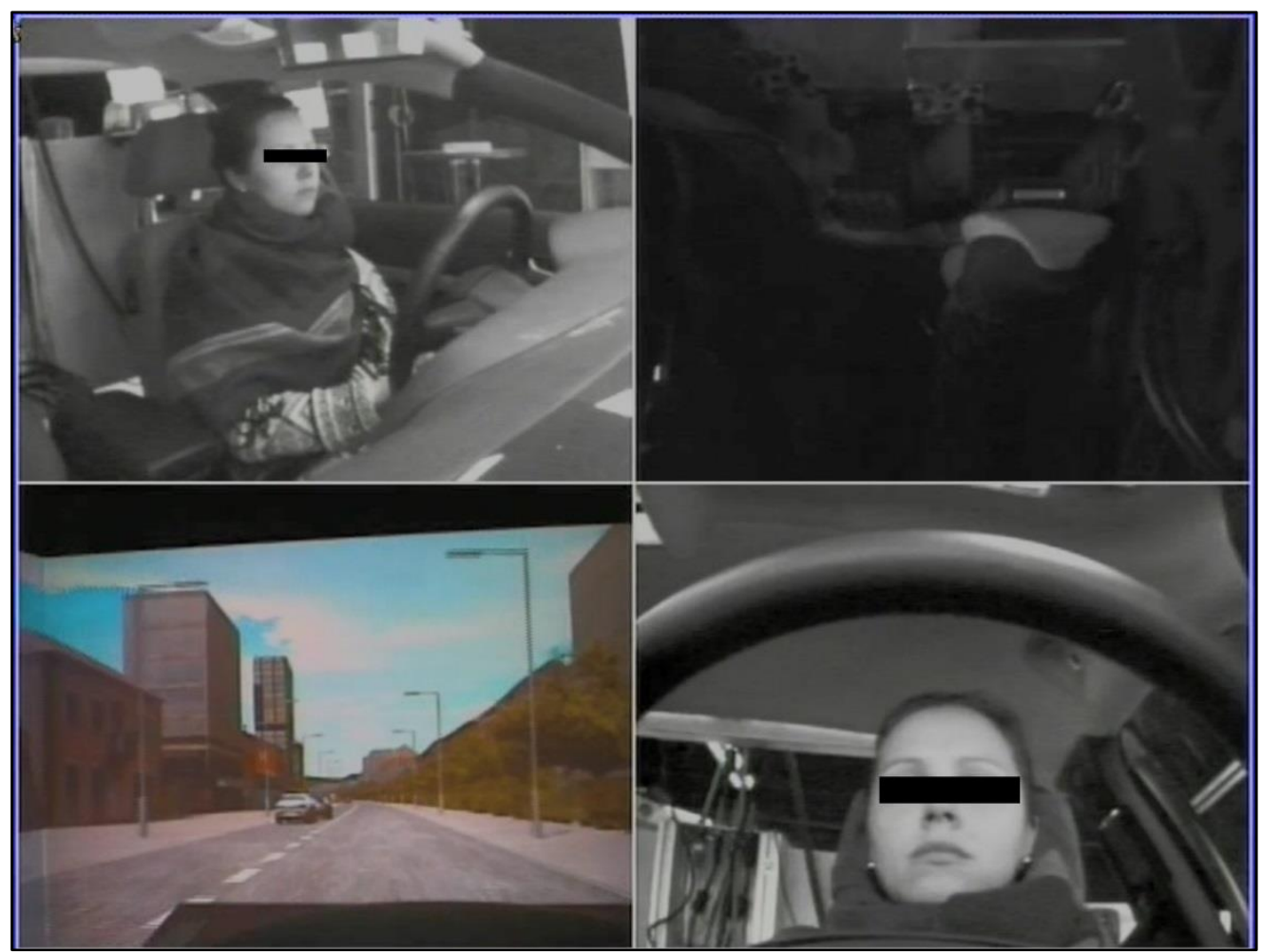

Abbildung 17: Ansicht integriertes System mit vier Kameras.
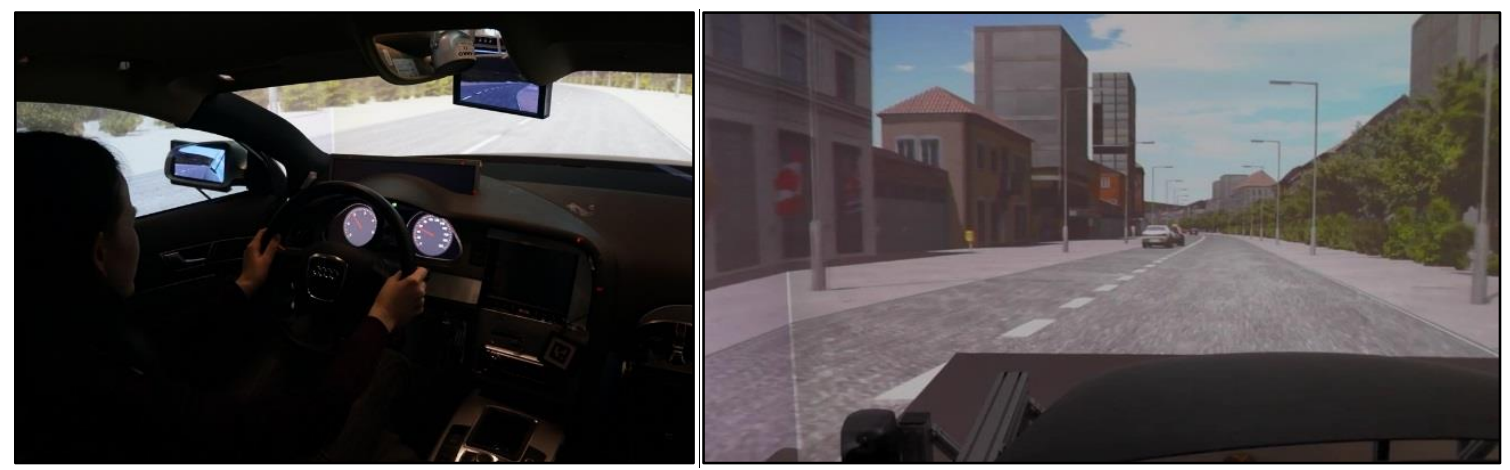

Abbildung 18: Ansicht Innenraum von „Rücksitz“ durch Innenraumkamera (links) und Ansicht der Strecke durch Szenenkamera aufgenommen (rechts).

\section{Szenarien der Fahrsimulation}

Es wurde eine abwechslungsreiche $11,7 \mathrm{~km}$ lange Strecke im urbanen Raum erstellt, deren Durchfahrung im Schnitt etwa 15 Minuten dauerte. Diese Strecke bestand aus zehn verschiedenen Szenarien. Zwei Szenarien beinhalteten kritische Situationen (siehe Abbildung 19): 
- Kind: Egofahrzeug ${ }^{15}$ fährt auf 2-spuriger Straße mit parkenden Fahrzeugen am rechten Fahrbahnrand, plötzlich tritt ein Kind hinter einem parkenden Fahrzeug hervor und überquert die Fahrbahn. In einer Variante rollt ein Ball als Vorinformation über die Straße bevor das Kind hinter dem parkenden Fahrzeug hervortrat, in der anderen Variante fehlt diese Vorinformation.

- Baustelle: Egofahrzeug fährt auf 2-spuriger Straße und nähert sich einer kaum sichtbaren Baustelle hinter einer leichten Kurve. In einer Variante dieser Situation wurde vor der Baustelle ein Hinweisschild als relevante Verkehrsinformation platziert, in der anderen Variante fehlt dieses Schild.

\section{Kind}
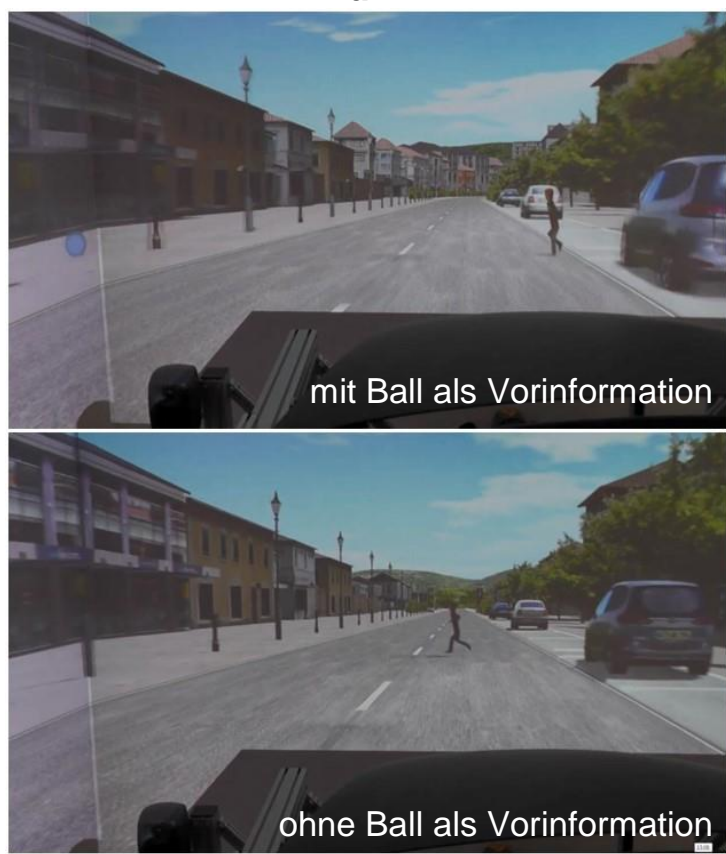

Baustelle

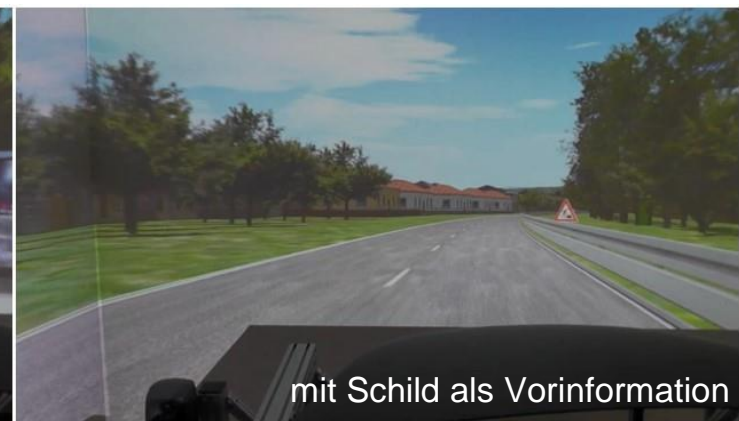

Äberblick über die Szenarien mit kritischen Situationen, oben mit Information als Vorinformation und unten ohne Information als Vorinformation, rechts Situationen mit dem überquerenden Kind, links mit der Baustelle.

In zwei weiteren Szenarien wurden den Fahrern relevante Verkehrsinformationen präsentiert (Schilder zur $30 \mathrm{~km} / \mathrm{h}$ Geschwindigkeitsbegrenzung, bei zuvor $50 \mathrm{~km} / \mathrm{h}$ ), bei denen zwar auch eine Reaktion des Fahrers erforderlich war, es aber, unabhängig vom Verhalten des Fahrers, in der Folge zu keiner kritischen Situation kam. Dies wurde zum einen bei einer Durchfahrt durch einen Park (Abbildung 20, links), zum anderen bei einer Fahrt im Wohn- und Geschäftsviertel eingesetzt (Abbildung 20, rechts). 

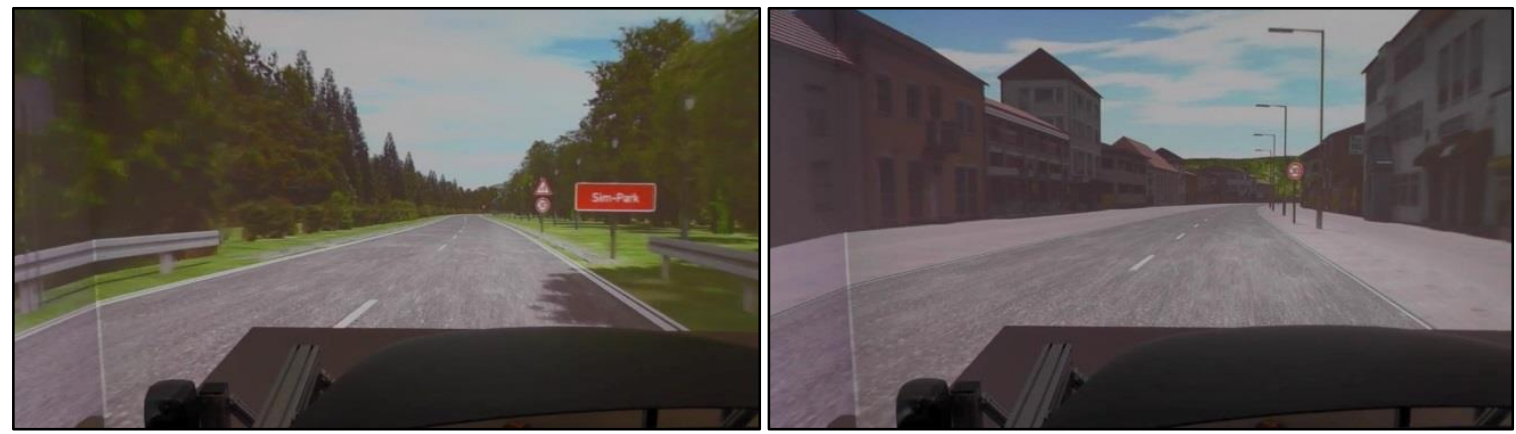

Abbildung 20: Überblick über die Szenarien mit relevanten Verkehrsinformationen (Schild $30 \mathrm{~km} / \mathrm{h}$ Geschwindigkeitsbegrenzung).

Zusätzlich zu diesen Szenarien bestand die Strecke noch aus sechs weiteren Abschnitten, bei denen typische „normale“ Fahraufgaben im urbanen Raum abgebildet wurden (genauere Beschreibung siehe Tabelle 15). Diese Szenarien dienten dazu den Fokus der Fahrer nicht zu stark auf die kritischen Situationen zu lenken.

In der Simulation waren sowohl Streckenabschnitte mit $30 \mathrm{~km} / \mathrm{h}$ als auch $50 \mathrm{~km} / \mathrm{h}$ Geschwindigkeitsbegrenzung implementiert. Die Fahrer durchfuhren diese Fahrsimulation zweimal. Zu diesem Zweck wurden zwei verschiedene Abläufe der Strecke programmiert, um zwischen der ersten und zweiten Fahrt Lerneffekte zu vermeiden. In den beiden Abläufen waren jeweils dieselben Szenarien in veränderter Anordnung enthalten. In Ablauf 1 wurde das Kind mit Ball als Vorinformation, aber die Baustelle ohne Hinweisschild präsentiert, während in Ablauf 2 das Kind ohne Ball als Vorinformation die Straße querte, aber vor der Baustelle ein Schild auf diese hinwies. Die Abfolge der Abläufe wurde über die Teilnehmer hinweg ausbalanciert. Der einen Hälfte der Teilnehmer wurde in der ersten Fahrt Ablauf 1 präsentiert, die andere Hälfte fuhr in der ersten Fahrt Ablauf 2. Ablauf 1 ist in Tabelle 15 exemplarisch dargestellt.

Tabelle 15: Überblick über die Szenarien der Fahrsimulation (Reihenfolge beispielhaft für Anordnung innerhalb der Simulation).

\begin{tabular}{|c|c|c|}
\hline Szenario & Spezifikation & Geschwindigkeit \\
\hline $\begin{array}{l}\text { 01. Anfahren, „normales“, } \\
\text { unkritisches Fahren }\end{array}$ & $\begin{array}{l}\text { Egofahrzeug startet aus Parklücke, ordnet } \\
\text { sich in Verkehr ein, fährt auf 2-spuriger } \\
\text { Straße innerhalb der Stadt }\end{array}$ & $\max .50 \mathrm{~km} / \mathrm{h}$ \\
\hline $\begin{array}{l}\text { 02. „Normales“, unkritisches } \\
\text { Fahren }\end{array}$ & $\begin{array}{l}\text { Egofahrzeug fährt auf 2-spuriger Straße, } \\
\text { Egofahrzug überquert verschiedene } \\
\text { Kreuzungen auf Vorfahrtsstraße ohne } \\
\text { Ampel, Umgebungsbedingungen in } \\
\text { Simulation allgemein: wenig } \\
\text { Umgebungsverkehr, teilweise kurvige } \\
\text { Strecke, wenige Fußgänger am } \\
\text { Fahrbahnrand, ab und zu Kreuzungen, } \\
\text { parkende Fahrzeuge, teilweise parkähnlich }\end{array}$ & $\max .50 \mathrm{~km} / \mathrm{h}$ \\
\hline $\begin{array}{l}\text { 03. Relevante } \\
\text { Verkehrsinformation, } \\
\text { kritische Situation in } \\
\text { der Folge }\end{array}$ & $\begin{array}{l}\text { Egofahrzeug fährt auf 2-spuriger Straße } \\
\text { mit parkenden Fahrzeugen am rechten } \\
\text { Fahrbahnrand, Ball rollt als } \\
\text { Vorinformation über die Straße bevor } \\
\text { das Kind hinter einem parkenden } \\
\text { Fahrzeug hervortritt und die Fahrbahn } \\
\text { überquert }\end{array}$ & $\max .50 \mathrm{~km} / \mathrm{h}$ \\
\hline
\end{tabular}




\begin{tabular}{|c|c|c|}
\hline Szenario & Spezifikation & Geschwindigkeit \\
\hline $\begin{array}{l}\text { 04. „Normales“, unkritisches } \\
\text { Fahren }\end{array}$ & $\begin{array}{l}\text { Egofahrzeug fährt auf 2-spuriger Straße, } \\
\text { überquert Ampelkreuzung auf mittlerer Spur } \\
\text { geradeaus, so dass vorher ein } \\
\text { Spurwechsel erforderlich ist, fährt weiter auf } \\
\text { 4-spuriger Straße und anschließend wieder } \\
\text { auf 2-spuriger Straße, leichte Kurven z.B. } \\
\text { Abbiegende Hauptstraße }\end{array}$ & $\max .50 \mathrm{~km} / \mathrm{h}$ \\
\hline $\begin{array}{l}\text { 05. Relevante } \\
\text { Verkehrsinformation, } \\
\text { keine kritische } \\
\text { Situation }\end{array}$ & $\begin{array}{l}\text { Egofahrzeug fährt auf 2-spuriger Straße, } \\
\text { Ankündigung Verringerung der } \\
\text { Geschwindigkeit auf } 30 \mathrm{~km} / \mathrm{h} \text {, danach } \\
\text { folgt Zone mit } 30 \mathrm{~km} / \mathrm{h} \text { Begrenzung }\end{array}$ & $\max .30 \mathrm{~km} / \mathrm{h}$ \\
\hline $\begin{array}{l}\text { 06. „Normales“, unkritisches } \\
\text { Fahren }\end{array}$ & $\begin{array}{l}\text { Egofahrzeug fährt auf einspuriger Straße in } \\
\text { 30er Zone mit parkenden Fahrzeugen am } \\
\text { Fahrbahnrand, muss an Kreuzung anhalten } \\
\text { und Vorfahrt gewähren, biegt anschließend } \\
\text { wieder in 2-spurige Straße ein, dann wieder } \\
50 \mathrm{~km} / \mathrm{h} \text { Begrenzung }\end{array}$ & $\max .30 \mathrm{~km} / \mathrm{h}$ \\
\hline $\begin{array}{l}\text { 07. Keine vorhergehenden } \\
\text { Informationen, kritische } \\
\text { Situation }\end{array}$ & $\begin{array}{l}\text { Egofahrzeug fährt auf 2-spuriger Straße, } \\
\text { schwer sichtbare Baustelle ohne } \\
\text { vorherige Ankündigung durch } \\
\text { Beschilderung nach leichter Kurve }\end{array}$ & $\max .50 \mathrm{~km} / \mathrm{h}$ \\
\hline $\begin{array}{l}\text { 08. „Normales“, unkritisches } \\
\text { Fahren }\end{array}$ & $\begin{array}{l}\text { 2-spurige Straße an Ampelkreuzung nach } \\
\text { Stopp überqueren in Mittelspur geradeaus, } \\
\text { 2-spurig weiter }\end{array}$ & $\max .50 \mathrm{~km} / \mathrm{h}$ \\
\hline $\begin{array}{l}\text { 09. Relevante } \\
\text { Verkehrsinformation, } \\
\text { keine kritische } \\
\text { Situation }\end{array}$ & $\begin{array}{l}\text { Egofahrzeug fährt auf 2-spuriger Straße, } \\
\text { Ankündigung Verringerung der } \\
\text { Geschwindigkeit auf } 30 \mathrm{~km} / \mathrm{h} \text {, danach } \\
\text { folgt Zone mit } 30 \mathrm{~km} / \mathrm{h} \mathrm{Begrenzung,} \\
\text { anschließend gilt wieder } 50 \mathrm{~km} / \mathrm{h} \\
\text { Begrenzung }\end{array}$ & $\max .30 \mathrm{~km} / \mathrm{h}$ \\
\hline $\begin{array}{l}\text { 10. Anhalten, „normales“, } \\
\text { unkritisches Fahren }\end{array}$ & $\begin{array}{l}\text { Egofahrzeug fährt auf 2-spuriger Straße und } \\
\text { hält am Fahrbahnrand }\end{array}$ & $\max .50 \mathrm{~km} / \mathrm{h}$ \\
\hline
\end{tabular}

\section{Nebentätigkeiten}

Während des Fahrens sollten die Teilnehmer an 10 Stellen in der Fahrsimulation Nebentätigkeiten bearbeiten. Insgesamt gab es vier verschiedene Nebentätigkeiten. Zwei Tätigkeiten erforderten eine visuell-manuelle Bedienung des Smartphones, für die anderen beiden wurde die Sprachsteuerung des im Fahrzeug integrierten Systems (Touchdisplay in der Mittelkonsole) genutzt. Jeder Teilnehmer führte im Versuch zwei dieser Tätigkeiten aus (jeweils eine Tätigkeit pro Fahrt), entweder die visuell-manuellen, oder die sprachbasierten.

\section{Visuell-manuelle Bedienung}

- Lesen: Teilnehmer lasen eine Textnachricht auf ihrem eigenen Smartphone während des Fahrens, welche innen vom Versuchsleiter zugeschickt wurde (siehe Abbildung 21 links). Der Eingang der Nachricht wurde durch ein akustisches Signal angekündigt. 
- Schreiben: Teilnehmer verfassten eine Textnachricht an ihrem Smartphone zu einem Thema, welches innen zuvor verbal (vom Band) vorgegeben wurde.

\section{Sprachbasierte Bedienung}

- Vorlesen: Auch hier wurde der Eingang der Nachricht durch ein akustisches Signal angekündigt. Die Teilnehmer mussten das System per Berührung einer Schaltfläche am Touchdisplay aktivieren, danach wurde innen die Nachricht von einer Computerstimme vorgelesen (siehe Abbildung 21 rechts).

- Spracheingabe: Es erfolgte eine verbale Vorgabe (vom Band) eines Themas für eine Textnachricht. Zum Einsprechen einer Nachricht musste zunächst die Schaltfläche auf dem Touchdisplay berührt werden. Ein Ton signalisierte die Aufnahmebereitschaft des Systems. Abschließend musste die Eingabe mit dem Sprachbefehl „Senden“ bestätigt werden.

In den Bedingungen „Lesen“ und „Vorlesen“ bekamen die Teilnehmer Textnachrichten mit einfachen Rätseln geschickt, welche sie mündlich beantworteten, z.B. „Du hast eine Nachricht von Frauke. Bitte entscheide, welches Wort passt nicht in die Reihe: Tennis Fußball - Volleyball - Handball - Schwimmen“. Die Abfolge der Rätsel war für alle Fahrer gleich. In den Bedingungen Schreiben und Spracheingabe wurde den Teilnehmern ein Thema auditiv vorgegeben, zu welchem sie eine Textnachricht schreiben oder einsprechen sollten, z.B. „gestern Abend“. Die Nachrichten sollten relativ kurz, d.h. i.d.R. einen Satz (5-6 Wörter) lang sein. Die Abfolge der Themen war für alle Fahrer gleich.

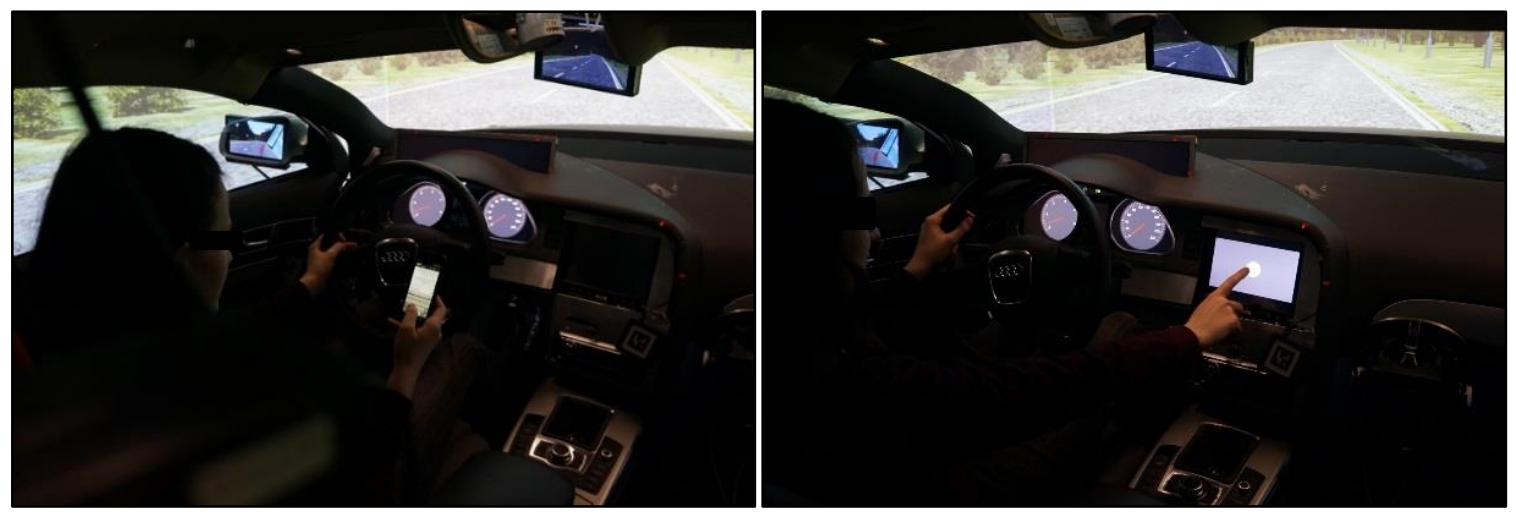

Abbildung 21: Bearbeitung der Nebentätigkeit am Smartphone (links) bzw. am Touchdisplay (rechts).

Aus den beiden Simulationsabläufen und den verschiedenen Nebentätigkeiten ergaben sich insgesamt zehn verschiedene Kombinationen, aufgeteilt auf die beiden Fahrten der Teilnehmer (siehe Tabelle 16). So las z.B. Teilnehmer Nr. 1 in der ersten Fahrt Textnachrichten am Smartphone und fuhr auf der Strecke, bei der der Ball als Vorinformation auf das Kind und die Baustelle ohne Hinweisschild präsentiert wurde. In Fahrt 2 verfasste er Textnachrichten am Smartphone und fuhr auf der Strecke, auf der die Baustelle mit Hinweisschild versehen war, aber das Kind ohne Vorinformation die Straße querte usw. (siehe Tabelle 16). 
Tabelle 16: Überblick über die verschiedenen Kombinationen für die Fahrten mit Simulationsabläufen und Nebentätigkeiten.

\begin{tabular}{|c|c|c|c|c|}
\hline \multirow{2}{*}{$\begin{array}{l}\text { Teil- } \\
\text { nehmer }\end{array}$} & \multicolumn{2}{|c|}{ Fahrt 1} & \multicolumn{2}{|c|}{ Fahrt 2} \\
\hline & Bedingung & Ablauf & Bedingung & Ablauf \\
\hline 001 & Lesen & $\begin{array}{l}\text { Ablauf } 1 \\
\text { Info + Kind } \\
\text { Baustelle }\end{array}$ & Schreiben & $\begin{array}{l}\text { Ablauf } 2 \\
\text { Info + Baustelle } \\
\text { Kind }\end{array}$ \\
\hline 002 & Lesen & $\begin{array}{l}\text { Ablauf } 2 \\
\text { Info + Baustelle } \\
\text { Kind }\end{array}$ & Schreiben & $\begin{array}{l}\text { Ablauf } 1 \\
\text { Info + Kind } \\
\text { Baustelle } \\
\end{array}$ \\
\hline 003 & Schreiben & $\begin{array}{l}\text { Ablauf } 1 \\
\text { Info + Kind } \\
\text { Baustelle }\end{array}$ & Lesen & $\begin{array}{l}\text { Ablauf } 2 \\
\text { Info + Baustelle } \\
\text { Kind }\end{array}$ \\
\hline 004 & Schreiben & $\begin{array}{l}\text { Ablauf } 2 \\
\text { Info + Baustelle } \\
\text { Kind }\end{array}$ & Lesen & $\begin{array}{l}\text { Ablauf } 1 \\
\text { Info + Kind } \\
\text { Baustelle } \\
\end{array}$ \\
\hline 005 & Vorlesen & $\begin{array}{l}\text { Ablauf } 1 \\
\text { Info + Kind } \\
\text { Baustelle } \\
\end{array}$ & Spracheingabe & $\begin{array}{l}\text { Ablauf } 2 \\
\text { Info + Baustelle } \\
\text { Kind } \\
\end{array}$ \\
\hline 006 & Vorlesen & $\begin{array}{l}\text { Ablauf } 2 \\
\text { Info + Baustelle } \\
\text { Kind }\end{array}$ & Spracheingabe & $\begin{array}{l}\text { Ablauf } 1 \\
\text { Info + Kind } \\
\text { Baustelle } \\
\end{array}$ \\
\hline 007 & Spracheingabe & $\begin{array}{l}\text { Ablauf } 1 \\
\text { Info + Kind } \\
\text { Baustelle }\end{array}$ & Vorlesen & $\begin{array}{l}\text { Ablauf } 2 \\
\text { Info + Baustelle } \\
\text { Kind }\end{array}$ \\
\hline 008 & Spracheingabe & $\begin{array}{l}\text { Ablauf } 2 \\
\text { Info + Baustelle } \\
\text { Kind }\end{array}$ & Vorlesen & $\begin{array}{l}\text { Ablauf } 1 \\
\text { Info + Kind } \\
\text { Baustelle }\end{array}$ \\
\hline 009 & ohne N & $\begin{array}{l}\text { Ablauf } 1 \\
\text { Info + Kind } \\
\text { Baustelle } \\
\end{array}$ & ohne NA & $\begin{array}{l}\text { Ablauf } 2 \\
\text { Info + Baustelle } \\
\text { Kind }\end{array}$ \\
\hline 010 & ohne NA & $\begin{array}{l}\text { Ablauf } 2 \\
\text { Info + Baustelle } \\
\text { Kind }\end{array}$ & ohne NA & $\begin{array}{l}\text { Ablauf } 1 \\
\text { Info + Kind } \\
\text { Baustelle }\end{array}$ \\
\hline$\ldots$ & $\ldots$ & & $\ldots$ & \\
\hline
\end{tabular}

\section{Beanspruchungsmessung und Interview zu ausgewählten Verkehrsszenen}

Um zu überprüfen, wie beanspruchend die Strecke von den Fahrern wirklich empfunden wird, wurden ausgewählte Szenarien hinsichtlich ihrer Beanspruchung bewertet. Dazu wurden sechs verschiedene Fahrsituationen mit einer Länge von jeweils zehn Sekunden aus der Simulation ausgewählt (Beschreibung siehe Tabelle 17). Die zugehörigen Videos wurden den Teilnehmern nach Abschluss der Fahrten präsentiert. Die Teilnehmer sollten zu jeder Situation berichten, wie stark beansprucht sie sich in dieser Situation während des Fahrens gefühlt haben. Etwaige Nebenaufgabenbearbeitungen in 
den jeweiligen Situationen sollten nicht in die Bewertung mit einbezogen werden. Die Beanspruchung wurde mittels Rating Scale for Mental Effort (RSME, Zijlstra 1985; Zijlstra 1993) von 0 - absolut keine Anstrengung bis 150 - mehr als extreme Anstrengung erfasst. Außerdem wurden den Teilnehmern zu diesen Situationen dieselben Fragen aus der Interviewstudie gestellt (siehe Kapitel 4.1.2), d.h. inwieweit sie in der jeweiligen Situation bereit wären Textnachrichten zu verfassen, welche situativen Aspekte maßgeblich für die (fehlende) Bereitschaft sind, welche Aspekte verändert werden müssten um diese Bereitschaft zu senken oder zu erhöhen, und wie sich das Unfallrisiko in der jeweiligen Situation verändert hätte, wenn sie mit dem Schreiben einer Textnachricht begonnen hätten.

Tabelle 17: Überblick über die sechs Fahrsituationen zur Beanspruchungsmessung.

\begin{tabular}{|c|c|}
\hline Situation & Beschreibung \\
\hline & $\begin{array}{l}\text { Egofahrzeug fährt auf zweispuriger Straße mit parkenden } \\
\text { Autos an beiden Fahrbahnrändern, ein Fahrzeug kommt } \\
\text { entgegen, Egofahrzeug kreuzt Fußgängerüberweg. }\end{array}$ \\
\hline & $\begin{array}{l}\text { Egofahrzeug fährt auf einspuriger Straße mit parkenden } \\
\text { Autos am linken Fahrbahnrand und Fußgängern auf dem } \\
\text { linken Gehweg, Egofahrzeug fährt auf eine T-Kreuzung zu }\end{array}$ \\
\hline & $\begin{array}{l}\text { Egofahrzeug fährt auf zweispuriger Straße mit parkenden } \\
\text { Autos am rechten Fahrbahnrand, die Straßenführung führt } \\
\text { aus einer Kurve mit zunächst wenig Einsicht auf eine } \\
\text { geradlinige Straße }\end{array}$ \\
\hline & $\begin{array}{l}\text { Egofahrzeug fährt auf einspuriger Straße mit parkenden } \\
\text { Autos am linken Fahrbahnrand und Fußgängern auf dem } \\
\text { linken Gehweg, Egofahrzeug fährt auf eine Kreuzung zu }\end{array}$ \\
\hline & $\begin{array}{l}\text { Egofahrzeug fährt auf zweispuriger, gerader Straße, mit } \\
\text { Leitplanke am rechten Fahrbahnrand und Wiese am linken } \\
\text { Fahrbahnrand auf eine Rechtskurve ohne Einsicht zu }\end{array}$ \\
\hline & $\begin{array}{l}\text { Egofahrzeug fährt auf dreispuriger Straße auf Ampelkreuzung } \\
\text { zu, muss sich in die mittlere Spur (Geradeausspur) } \\
\text { einordnen, Ampel schaltet von Rot auf Grün, an der } \\
\text { Kreuzung kommt ein Fahrzeug entgegen }\end{array}$ \\
\hline
\end{tabular}


Analog zur Interviewstudie wurde ein Fragebogen zur Erfassung von überdauernden Persönlichkeitsmerkmalen eingesetzt. Unter anderem wurden die Einstellung gegenüber dem Schreiben von Textnachrichten (Harrison, 2011) und das gewohnheitsmäßige Schreiben von Textnachrichten (Bayer \& Campbell, 2012) erfasst. Außerdem wurden in Anlehnung an die Theorie des geplanten Verhaltens die subjektive Norme, Einstellungen und Verhaltenskontrolle über das Texten während des Fahrens erhoben (Bayer \& Campbell, 2012). Als fahrbezogene Variable wurde der Fahrstil der Teilnehmer abgefragt (Popken, 2009).

\subsubsection{Ablauf}

Nach der Begrüßung durch den Versuchsleiter erhielten die Teilnehmer eine kurze Einführung zur Fahrsimulatorstudie, sowie eine Aufklärung über den Simulator, die Simulatorkrankheit und darüber, dass Video- und Audiodaten aufgezeichnet werden. Anschließend absolvierten sie eine kurze Übungsfahrt im Fahrsimulator, damit sie sich an die Steuerung gewöhnen konnten. Danach folgten die jeweiligen Instruktionen für die Fahrt. Aufgabe der Teilnehmer war es, innerhalb der Simulation den Ausschilderungen Richtung Chemnitz zu folgen, so natürlich wie möglich zu fahren und sich dabei an die Verkehrsregeln halten. Sie sollten nicht in Eile sein, aber auch nicht zu langsam fahren.

Zusätzlich erfolgte die Einweisung zur Bearbeitung der Nebentätigkeiten. Die Teilnehmer der Kontrollgruppe, die keiner Nebentätigkeit nachgehen sollten, erhielten nur die Instruktionen für die Fahrt. Bei allen Nebentätigkeiten wurde erläutert, dass ein Ton die Möglichkeit zur Bearbeitung der Nebentätigkeiten signalisiert. Die Teilnehmer sollten dann selbst entscheiden, ob die Situation es ihrer Meinung nach erlaubt eine Nebentätigkeit zu bearbeiten. Sie sollten sich nicht gezwungen fühlen in der Situation die Nebentätigkeit aufzunehmen und ganz so entscheiden, wie sie es sonst im Straßenverkehr auch tun würden. Außerdem wurden spezifische Instruktionen zur jeweiligen Nebentätigkeit erteilt, z.B. über das Berühren des Touchdisplays oder die Funktionsweise der Spracheingabe. Außerdem wurde darüber informiert, dass 30 Sekunden nach dem Startton zur Bearbeitung ein weiteres auditives Signal ertönen würde welches anzeigt, dass die Bearbeitung der Nebenaufgabe beendet werden sollte, egal welchen Stand die Bearbeitung hat. Damit sollte vermieden werden, dass die Teilnehmer zu lange mit einer Nebentätigkeit beschäftigt sind, was Probleme für das Timing der folgenden Aufgaben mit sich gebracht hätte.

Nach den Instruktionen absolvierten die Teilnehmer die erste Versuchsfahrt im Simulator. Danach füllten sie den ersten Teil des Fragebogens aus. Zweck dieser Unterbrechung war es, die Teilnehmer dazu zu bringen den Fahrsimulator zu verlassen und tatsächlich eine kurze Pause einzulegen, um u.a. auch der Simulatorkrankheit vorzubeugen. Anschließend wurden die Instruktionen zur zweiten Fahrt und den jeweiligen Nebentätigkeiten gegeben, gefolgt von der Fahrt. Nachdem beide Fahrten abgeschlossen waren, wurden den Teilnehmern die Verkehrsszenen aus der Simulation zur Beanspruchungsmessung vorgespielt. Es erfolgte die Situationsbewertung mittels RSME, Fragen zum Textingverhalten und den damit verknüpften situativen Merkmalen, sowie die Risikobewertung. Abschließend füllten sie den zweiten Teil des Fragebogens aus und wurden verabschiedet. Insgesamt dauerte der Versuch 90 bis 120 Minuten. 


\subsubsection{Datenaufbereitung und -analyse}

\section{Allgemeine Aspekte der Datenaufbereitung}

In einem ersten Schritt wurde zunächst die Verwertbarkeit der Daten, sowie die Synchronisierung der Daten aus verschiedenen Datenquellen sichergestellt. Für jeden Teilnehmer lagen Videodaten der verschiedenen Kameras sowie Fahrdaten in der Rohfassung aus dem Simulator vor. Ferner standen Interviewdaten inkl. Beanspruchungsmessung und Fragebogendaten zur Verfügung. Einen Überblick über die einzelnen Datentypen und die Schritte der Datenaufbereitung liefert Abbildung 22.

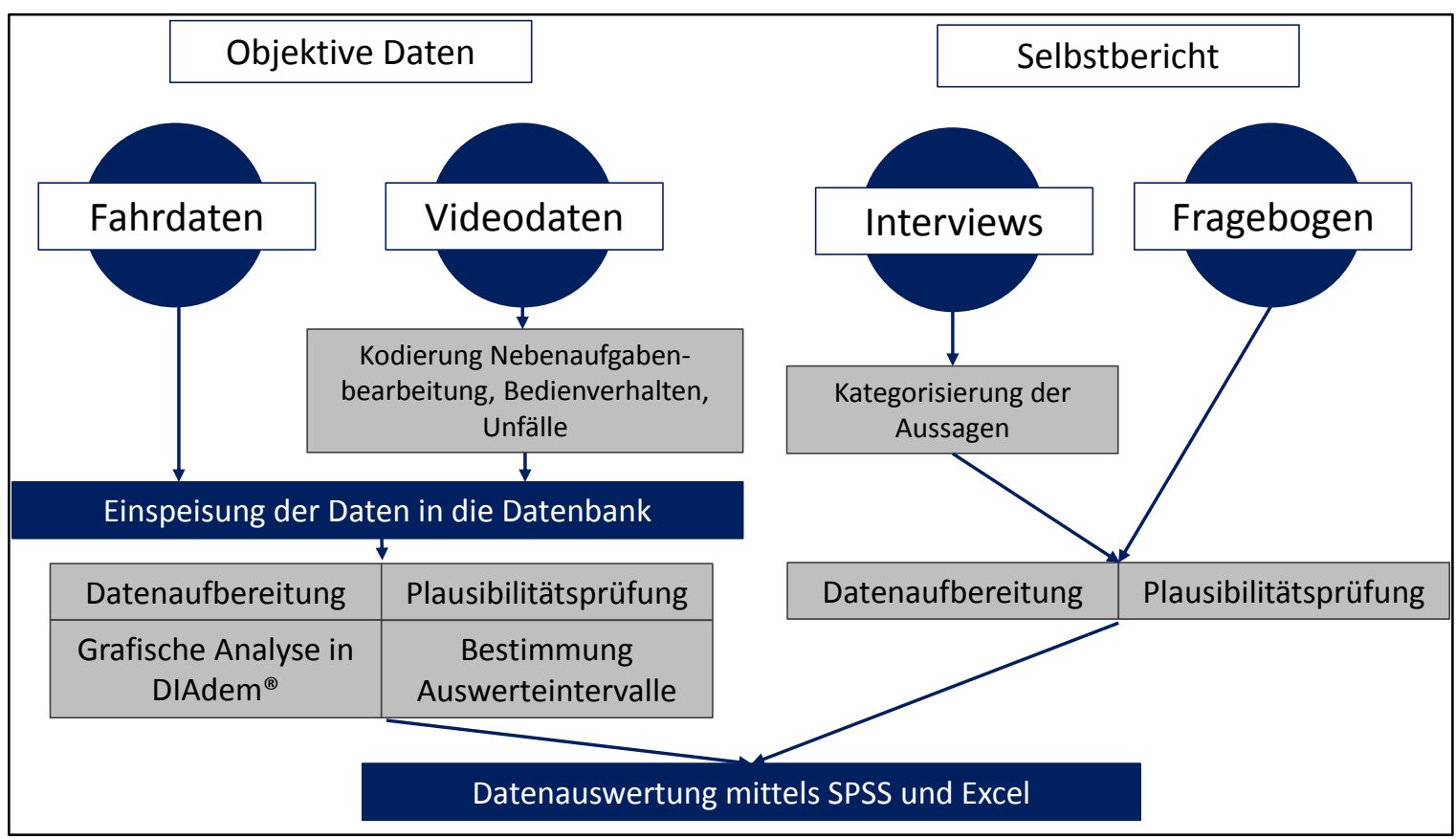

Abbildung 22: Überblick über die Datenquellen und Schritte zur Datenaufbereitung.

Zunächst wurden die Videos und Fahrdaten einander zugeordnet, um für jede Fahrt eines Teilnehmers einen möglichst vollständigen Datensatz zu erhalten. Die Fahrdaten wurden in eine Datenbank übertragen und anschließend umfangreichen Plausibilitätsprüfungen unterzogen, z.B. hinsichtlich Lücken in den Fahrdaten als auch Videos um ggf. Datensätze auszusortieren. Es wurden nur vollständige Datensätze berücksichtigt. Danach wurden sämtliche Videos so geschnitten, dass deren Beginn mit dem Start der Fahrt übereinstimmte. So konnte eine vollständige Synchronisierung mit den Fahrdaten erreicht werden. Diese Synchronisierung wurde mittels grafischer Analyse des Fahrverhaltens an markanten Streckenpunkten z.B. der kritischen Situationen mit Hilfe des Programms DIAdem® überprüft. Außerdem wurden an dieser Stelle die verschiedenen Fahrverhaltensparameter auf Ausreißer und unplausible Werte überprüft.

\section{Datenaufbereitung und -analyse der Dauer und Länge der Nebentätigkeiten}

Um die Bearbeitung der Nebentätigkeiten zu analysieren, wurden die Videodaten umfassend kodiert. Für die Kodierung der Dauer der Nebentätigkeit sowie des Bedienverhaltens am Mobiltelefon wurden vor allem die Videos der Innenraumkamera genutzt. Der Start einer Nebentätigkeit wurde definiert als das Lösen der Hand vom Lenkrad oder dem angestammten Platz (z.B. dem Oberschenkel) um nach dem Mobiltelefon bzw. dem Touchdisplay zu greifen. Die Nebentätigkeit war abgeschlossen, 
wenn sich die Hand wieder zurück am Lenkrad oder ihrem angestammten Platz befand (visuell-manuelle Bedienung) bzw. das letzte Wort gesprochen wurde (Sprachsteuerung). Zum Vergleich der Dauer der Nebentätigkeiten wurden inferenzstatistische Verfahren eingesetzt (Varianzanalyse).

Für die Länge der verfassten Nachrichten wurden jeweils die Anzahl der Zeichen inkl. Leerzeichen gezählt. Dabei ist zu beachten, dass bei diesem Maß lediglich das „Endergebnis“ der Eingaben betrachtet wird, etwaige zusätzliche Eingaben (z.B. bei Korrektur von Rechtschreibfehlern bei der visuell-manuellen Bedienung) wurden nicht erfasst. Bei der Spracheingabe wurde eine Audioaufzeichnung der eingesprochenen Nachrichten vorgenommen. Die Aussagen der Teilnehmer wurden verschriftlicht, um die Länge der Nachricht zu ermitteln. Damit entspricht die erfasste Länge derjenigen, die bei einem perfekten System (d.h. Spracherkennung ohne Fehler) zu erreichen wäre.

Datenaufbereitung und -analyse zum Einfluss der Bearbeitung der Nebentätigkeit auf allgemeine Fahrperformanz

Die Analyse der allgemeinen Fahrperformanz dient der Bewertung des Verhaltens bzw. der Verhaltenskonsequenzen der Nebentätigkeitsbearbeitung in nichtkritischen Verkehrssituationen. Für diese Analysen wurden für jeden Teilnehmer vergleichbare Streckenabschnitte (gerade, gleich lang) ausgewählt. Auf einigen dieser Abschnitte wurde eine Nebentätigkeit ausgeführt, auf anderen nicht. Es wurden jeweils zwei Abschnitte (einer mit, einer ohne Nebentätigkeit) mit Geschwindigkeitsbegrenzung $30 \mathrm{~km} / \mathrm{h}$ und Geschwindigkeitsbegrenzung $50 \mathrm{~km} / \mathrm{h}$ gewählt. Bei $30 \mathrm{~km} / \mathrm{h}$ Begrenzung waren die beiden ausgewählten Abschnitte jeweils $138 \mathrm{~m}$ lang, bei $50 \mathrm{~km} / \mathrm{h}$ Begrenzung $245 \mathrm{~m}$. Die tatsächlich sind jedoch in den meisten Fällen kürzer als die hier beschriebenen Maximallängen (138 m und $245 \mathrm{~m}$ ). Bei den Abschnitten bei denen eine Nebentätigkeit ausgeführt wurde, wurde dies selten über die gesamte Streckenlänge getan. Deshalb wurde innerhalb dieser Abschnitte für jeden Teilnehmer individuell die Strecke ermittelt, auf der tatsächlich eine Nebenaufgabenbearbeitung erfolgte. Diese Streckenlänge wurde letztlich für die Auswertung herangezogen, und auch auf den jeweils zugehörigen Abschnitt ohne Nebentätigkeit übertragen, so dass für jeden Teilnehmer eine individuelle Länge für die verglichenen Abschnitte mit $30 \mathrm{~km} / \mathrm{h}$ Begrenzung und für die Abschnitte mit $50 \mathrm{~km} / \mathrm{h}$ resultierte (siehe Abbildung 23: Exemplarische Darstellung der Ermittlung der Intervalle für einen Teilnehmer bei $50 \mathrm{~km} / \mathrm{h}$ Geschwindigkeitsbegrenzung. Abbildung 23).

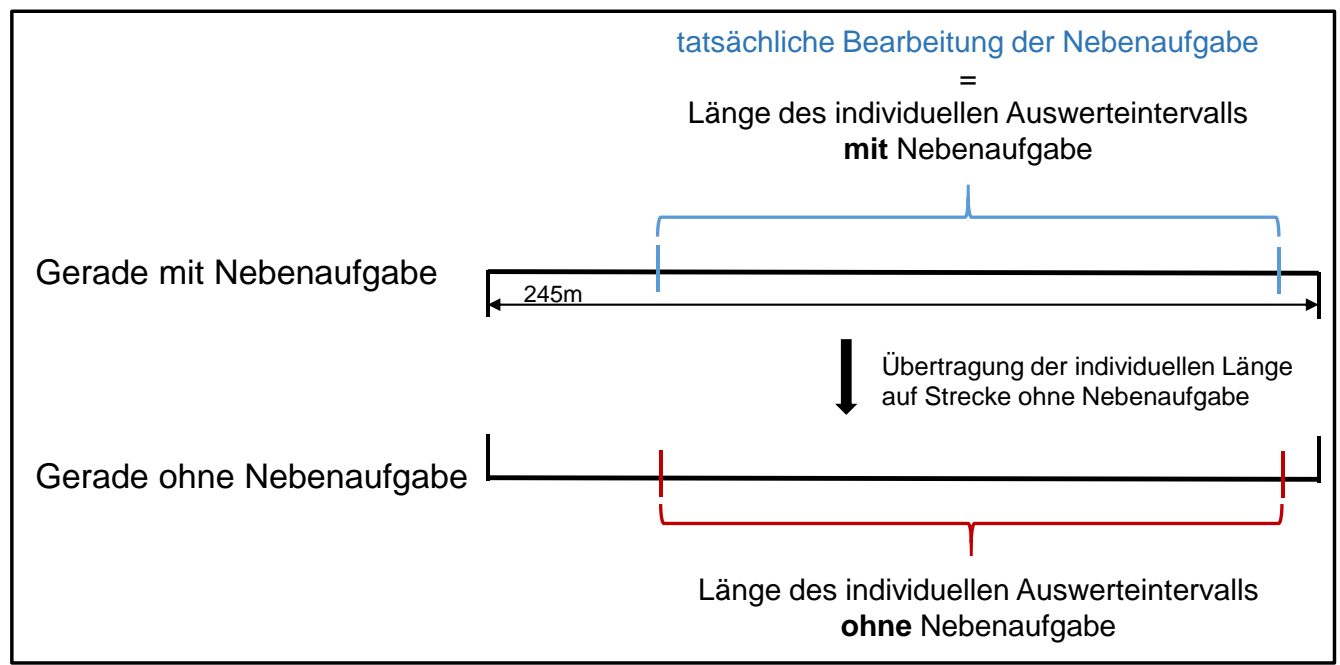

Abbildung 23: Exemplarische Darstellung der Ermittlung der Intervalle für einen Teilnehmer bei $50 \mathrm{~km} / \mathrm{h}$ Geschwindigkeitsbegrenzung. 
Für die Teilnehmer der Baselinebedingung (generell ohne Nebentätigkeit) wurde der Mittelwert der individuellen Vergleichsgeraden aller Teilnehmer mit Nebentätigkeit ermittelt. Dieser Mittelwert wurde genutzt um die Länge der zu analysierenden geraden Streckenabschnitte zu bestimmen.

Um Unterschiede zwischen den einzelnen Nebentätigkeiten hinsichtlich des Fahrverhaltens zu identifizieren, wurden die Durchschnittsgeschwindigkeiten sowie die Abweichungen in der lateralen Position betrachtet, und statistisch jeweils mit Hilfe von Varianzanalysen mit Messwiederholung (ANOVA) verglichen. Bei signifikanten Effekten wurden post-hoc Einzelvergleiche mit Bonferroni-Korrektur vorgenommen.

\section{Datenaufbereitung und -analyse der besonderen Verkehrssituationen Kind und Baustelle}

Analog zur Analyse der allgemeinen Fahrperformanz mussten auch für die Analyse der kritischen Szenarien zunächst die Streckenabschnitte definiert werden, innerhalb derer verschiedene Verhaltensparameter betrachtet werden sollten. Als Startpunkt eines Abschnittes wurde jeweils der Punkt gewählt, an dem die Vorinformation, also das Baustellenschild oder der Ball, sichtbar wurde. Für die Szenarien, in denen die kritische Situation ohne Vorinformation auftrat, wurde analog der Punkt gewählt, an dem diese Vorinformation sichtbar geworden wäre. Als Endpunkt wurde jeweils die Höhe des Kindes bzw. der Baustelle gewählt. Einen Überblick über die Länge der ausgewerteten Streckenabschnitte und auch der ausgewerteten Variablen gibt Tabelle 18.

Tabelle 18: Überblick über Länge der Auswerteintervalle und die ausgewerteten Variablen der einzelnen Szenarien.

\begin{tabular}{|c|c|c|c|}
\hline Szenario & Situation & Variablen & Länge Intervall \\
\hline $\begin{array}{l}\text { (Mit) / ohne } \\
\text { relevante } \\
\text { Information \& } \\
\text { kritische Situation }\end{array}$ & $\begin{array}{l}\text { (Ball rollt auf die } \\
\text { Straße) Kind tritt } \\
\text { hinter einem } \\
\text { parkenden } \\
\text { Fahrzeug } \\
\text { hervortritt und } \\
\text { überquert die } \\
\text { Fahrbahn }\end{array}$ & $\begin{array}{l}\text { - Reaktionszeit bei Reduktion } \\
\text { des Gaspedaldrucks bzw. } \\
\text { Betätigung des Bremspedals } \\
\text { ab Sichtbarkeit des Balls } \\
\text { - Abstand zum Kind } \\
\text { - Time to Collision zu Kind }\end{array}$ & $\begin{array}{l}70 \text { m (Sichtbarkeit } \\
\text { des Balls bis zur } \\
\text { Höhe des Kindes) }\end{array}$ \\
\hline $\begin{array}{l}\text { (Mit) / ohne } \\
\text { relevante } \\
\text { Information \& } \\
\text { kritische Situation }\end{array}$ & $\begin{array}{l}\text { (Baustellenschild) } \\
\text { Baustelle taucht } \\
\text { hinter Kurve auf }\end{array}$ & $\begin{array}{l}\text { - Reaktionszeit bei Reduktion } \\
\text { des Gaspedaldrucks bzw. } \\
\text { Betätigung des Bremspedals } \\
\text { ab Sichtbarkeit des } \\
\text { Baustellenschilds } \\
\text { - Abstand zur Baustelle } \\
\text { - Time to Collision zur Baustelle }\end{array}$ & $\begin{array}{l}270 \text { m (Sichtbarkeit } \\
\text { des Baustellen- } \\
\text { schilds bis zur } 1 . \\
\text { Pylone der } \\
\text { Baustelle) }\end{array}$ \\
\hline $\begin{array}{l}\text { Relevante } \\
\text { Information, } \\
\text { KEINE kritische } \\
\text { Situation }\end{array}$ & $\begin{array}{l}30 \mathrm{~km} / \mathrm{h} \text { Schild zur } \\
\text { Geschwindigkeits- } \\
\text { begrenzung im } \\
\text { Wohn- und } \\
\text { Geschäftsviertel }\end{array}$ & $\begin{array}{l}\text { - Reaktionszeit bei Reduktion } \\
\text { des Gaspedaldrucks bzw. } \\
\text { Betätigung des Bremspedals } \\
\text { ab Sichtbarkeit des } 30 \mathrm{~km} / \mathrm{h} \\
\text { Schildes } \\
\text { - Dauer bis eine } \\
\text { Geschwindigkeitsreduktion } \\
\text { von min. 20\% erreicht ist ab } \\
\text { Reaktion } \\
\text { - Geschwindigkeitsdifferenz ab } \\
\text { Sichtbarkeit des Schildes bis } \\
\text { zur Minimalgeschwindigkeit }\end{array}$ & $\begin{array}{l}380 \mathrm{~m} \\
\text { (Sichtbarkeit } \\
30 \mathrm{~km} / \mathrm{h} \text { Schild bis } \\
\text { zur nächsten Kurve) }\end{array}$ \\
\hline
\end{tabular}




\begin{tabular}{|c|c|c|c|}
\hline Szenario & Situation & Variablen & Länge Intervall \\
\hline $\begin{array}{l}\text { Relevante } \\
\text { Information, } \\
\text { KEINE kritische } \\
\text { Situation }\end{array}$ & $\begin{array}{l}30 \mathrm{~km} / \mathrm{h} \text { Schild zur } \\
\text { Geschwindigkeits- } \\
\text { begrenzung im } \\
\text { Parkareal }\end{array}$ & $\begin{array}{l}\text { - Reaktionszeit bei Reduktion } \\
\text { des Gaspedaldrucks bzw. } \\
\text { Betätigung des Bremspedals } \\
\text { ab Sichtbarkeit des } 30 \mathrm{~km} / \mathrm{h} \\
\text { Schildes } \\
\text { - Dauer bis eine } \\
\text { Geschwindigkeitsreduktion } \\
\text { von mindestens } 20 \% \text { erreicht } \\
\text { ab Reaktion } \\
\text { - Geschwindigkeitsdifferenz ab } \\
\text { Sichtbarkeit des Schildes bis } \\
\text { zur Minimalgeschwindigkeit }\end{array}$ & $\begin{array}{l}245 \mathrm{~m} \\
\text { (Sichtbarkeit } \\
30 \mathrm{~km} / \mathrm{h} \text { Schild bis } \\
\text { zur nächsten Kurve) }\end{array}$ \\
\hline
\end{tabular}

Bei der Analyse wurde schwerpunktmäßig das Verhalten bei kritischen Situationen mit und ohne Vorinformation in Abhängigkeit von den jeweils bearbeiteten Nebentätigkeiten betrachtet. Folgende Fahrverhaltensparameter wurden pro Situation ausgewertet:

- Anzahl Unfälle / Beinaheunfälle

- Reaktionszeit bis Reduktion des Gaspedaldrucks um mindestens 30\% / Betätigung des Bremspedals

- Abstand und Time to Collision (TTC) zu Kind / zu Baustelle im Moment der Reduktion des Gaspedaldrucks um mindestens 30\% / Betätigung des Bremspedals.

Die Videos des integrierten Kamerasystems wurden genutzt um Zusammenstöße mit dem Kind und der Baustelle zu identifizieren bzw. deren Häufigkeit zu bestimmen. Die Reaktionszeit wurde definiert als Zeit zwischen dem Einfahren in den analysierten Streckenabschnitt (d.h. Punkt an dem Vorinformation sichtbar wurde / sichtbar geworden wäre) und der Reduktion des Gaspedaldrucks bzw. der Bremspedalbetätigung. Ferner wurde der Abstand und die Time to Collision zum Kind oder der Baustelle im Moment der Reduktion des Gaspedaldrucks / Betätigung des Bremspedals bestimmt. Um Unterschiede zwischen der Bedingung mit und ohne Vorinformation und den einzelnen Nebentätigkeiten zu identifizieren, wurden jeweils gemischte Varianzanalysen (mixed ANOVA) berechnet. Bei signifikanten Effekten wurden post-hoc Einzelvergleiche mit Bonferroni-Korrektur vorgenommen. Bei den einzelnen Berechnungen liegen teilweise unterschiedliche Fallzahlen zu Grunde, da unterschiedlich viele Teilnehmer die Nebenaufgabe bearbeiteten bzw. nicht alle bremsten oder den Gaspedaldruck um mindestens $30 \%$ reduzierten.

Wenn die Voraussetzungen für eine Varianzanalyse verletzt waren (z.B. Normalverteilung und Varianzhomogenität bzw. Sphärizität), wurden nonparametrische Tests eingesetzt (Mann-Whitney-U-Test für zwei unabhängige Stichproben, KruskallWallis-H-Test für mehr als zwei unabhängige Stichproben). Bei signifikanten Effekten beim Vergleich von mehr als zwei Gruppen wurden anschließend post-hoc Tests mit Dunn-Bonferroni-Korrektur vorgenommen. Da insbesondere Reaktionszeiten selten normalverteilt sind, wurden in den Ergebnistabellen zusätzlich zu Mittelwert und Standardabweichung auch die Mediane dargestellt, da diese weniger anfällig für Ausreißer und Verzerrungen sind als Mittelwerte.

Datenanalyse der besonderen Verkehrssituationen Schild $30 \mathrm{~km} / \mathrm{h}$ Geschwindigkeitsbegrenzung

Auch für die Analyse der Reaktion auf die veränderte Geschwindigkeitsbegrenzung wurden zunächst die zu betrachtenden Streckenabschnitte definiert. Als Startpunkt 
wurde die Sichtbarkeit des Schildes zur $30 \mathrm{~km} / \mathrm{h}$ Geschwindigkeitsbegrenzung genutzt. Der Endpunkt wurde $180 \mathrm{~m}$ nach dem Schild gesetzt (maximal möglich, weil dann Kurve), da eine Reaktion auch erst nach dem Schild erfolgen konnte (siehe Tabelle 18). Für die beiden Szenarien wurden Vergleiche zwischen den verschiedenen Nebentätigkeiten hinsichtlich folgender Fahrverhaltensaspekte vorgenommen:

- Reaktionszeit bis Reduktion des Gaspedaldrucks um mindestens 30\% / Betätigung des Bremspedals

- Zeitspanne bis eine Geschwindigkeitsreduktion von minus 20\% nach der Reduktion des Gaspedaldrucks / Betätigung des Bremspedals erreicht wurde.

Die Reaktionszeit wurde als Zeit zwischen der Sichtbarkeit der $30 \mathrm{~km} / \mathrm{h}$ Begrenzung und der Reduktion des Gaspedaldrucks bzw. des Bremsens definiert. Ferner wurde die Zeitspanne bis zur Erreichung einer Geschwindigkeitsreduktion um mindestens 20\% (z.B. von $50 \mathrm{~km} / \mathrm{h}$ auf $40 \mathrm{~km} / \mathrm{h}$ ) ausgewertet. Hierfür wurde als Startpunkt der Zeitpunkt der Reduktion des Gaspedaldrucks bzw. die Betätigung des Bremspedals herangezogen. Um statistisch relevante Unterschiede zwischen den Nebentätigkeiten zu identifizieren wurden jeweils Varianzanalysen (ANOVA) und post-hoc Einzelvergleiche (mit Bonferroni-Korrektur) eingesetzt. Auch hier wurden im Falle der Verletzung der Voraussetzungen nonparametrische Tests eingesetzt (Mann-Whitney-U-Test für zwei unabhängige Stichproben, Kruskall-Wallis-H-Test für mehr als zwei unabhängige Stichproben).

\section{Datenaufbereitung und -analyse der Interviewdaten}

Die Audioaufnahmen des Interviews wurden vollständig nachgehört und einzelne Argumente bzw. wortwörtliche Aussagen der Personen transkribiert. Insgesamt wurden 664 Argumente registriert für die Frage warum die Fahrer in dieser Situation mit dem Schreiben von Textnachrichten beginnen würden. Insgesamt 343 Argumente gegen das Verfassen von Textnachrichten wurden erfasst. Für die Auswertung wurde, wie in der videobasierten, strukturierten Interviewstudie (vgl. Kapitel 4), eine qualitative Inhaltsanalyse nach Mayring (2000) durchgeführt. Die einzelnen Argumente wurden in mehreren Einzelschritten zu größeren Kategorien zusammengefasst. Diese wurden überarbeitet und verdichtet, und anschließend bezogen auf die Häufigkeit der Nennungen ausgewertet. Die komplementäre Frage, was in der jeweiligen Situation anders sein müsste, damit die Teilnehmer das Schreiben einer Textnachricht unterlassen würden (wenn sie vorher eine Textnachricht geschrieben hätten) oder auch damit beginnen (wenn sie es vorher unterlassen hätten), wurde ebenso ausgewertet. Für die Frage zur Unterlassung wurden 545 Argumente ausgewertet, während es für die Frage „Was müsste anders sein, damit Sie texten?" 264 Argumente waren.

\subsection{Ergebnisse}

Zuerst werden die Angaben zur Häufigkeit des Lesens und Schreibens von Textnachrichten (Kapitel 5.2.1), sowie Einstellungen und Persönlichkeitseigenschaften aus dem Fragebogen zur Beschreibung der Stichprobe dargestellt (Kapitel 5.2.2). Anschließend folgt ein Überblick über die Ergebnisse zur Fahrperformanz mit und ohne Nebentätigkeit (Kapitel 5.2.4). Danach werden die Ergebnisse für die Szenarien mit den kritischen Situationen (Kind/Baustelle) und die besonderen Verkehrssituationen (Schilder Geschwindigkeitsbegrenzung $30 \mathrm{~km} / \mathrm{h}$ ) dargestellt (Kapitel 5.2.5, 5.2.6, 5.2.7). Zum Abschluss folgen die Resultate der Beanspruchungsmessung und die Ergebnisse des Interviews zu einzelnen Verkehrsszenen aus der Simulation (Kapitel 5.2.8). 


\subsubsection{Häufigkeit des Lesens und Schreibens von Textnachrichten aus Fragebogendaten}

In Abbildung 24 ist die Häufigkeit des Lesens von Textnachrichten beim Fahren der Nutzung der Vorlesefunktion gegenübergestellt. Die Teilnehmer berichteten relativ hohe Nutzungsraten für das Lesen am Display ihres Mobiltelefons, während dagegen nur wenige Teilnehmer die Vorlesefunktion nutzten.

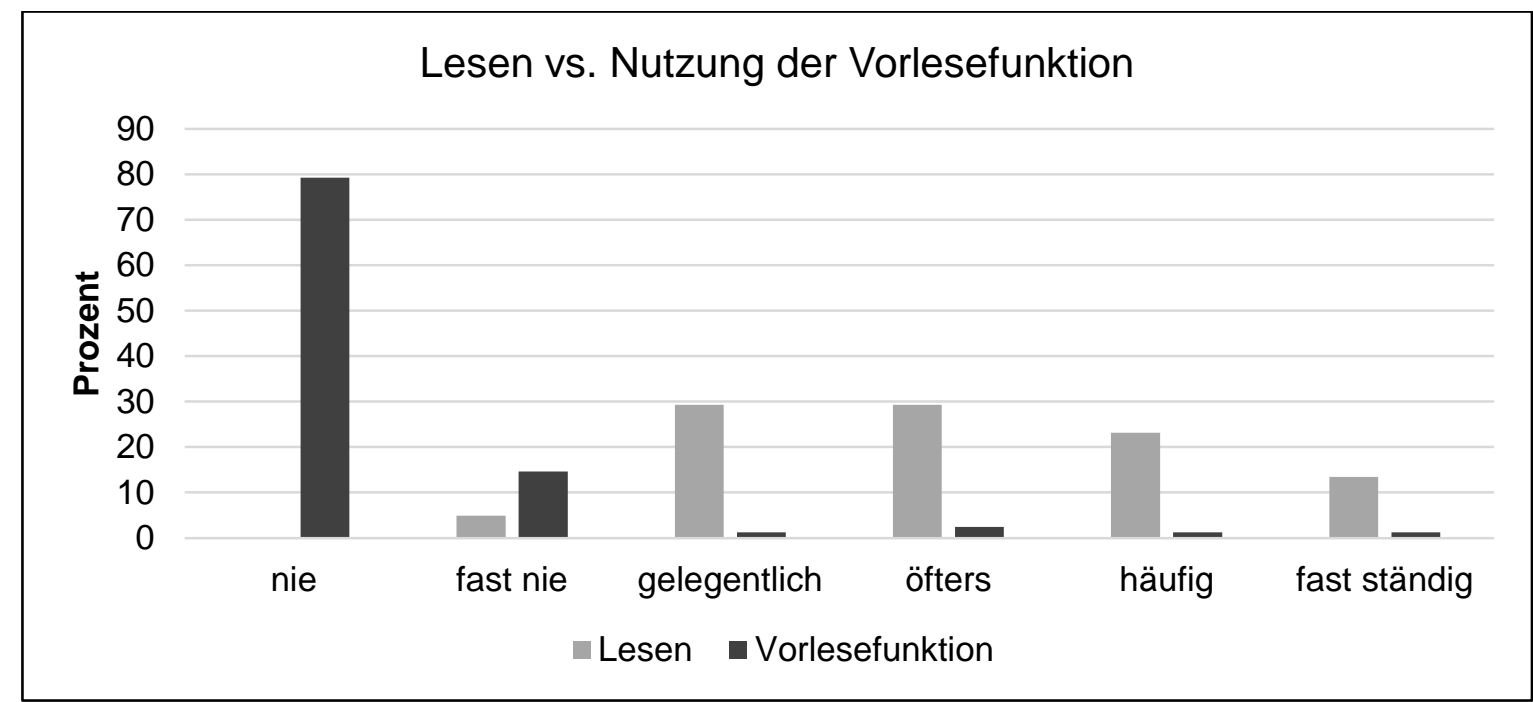

Abbildung 24: Häufigkeit des Lesens von Textnachrichten bzw. der Nutzung Vorlesefunktion während des Fahrens, Fragebogendaten $(N=82)$.

Für die Häufigkeit des Schreibens von Textnachrichten manuell am Mobiltelefon zeigt sich ein ähnliches Bild. Der überwiegende Teil der Teilnehmer gibt an, dies zumindest gelegentlich (oder häufiger) zu tun (siehe Abbildung 25), während die Spracheingabe selten bis nie genutzt wird.

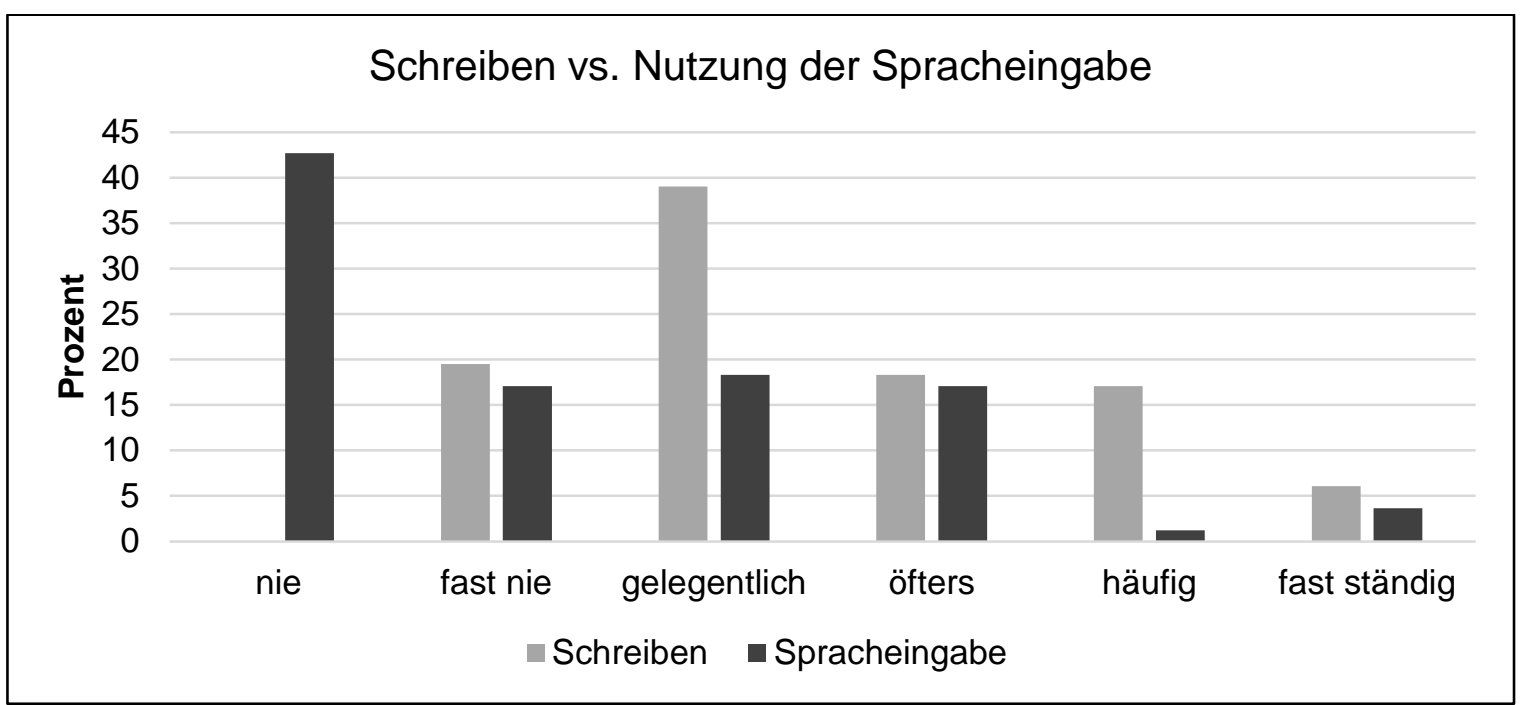

Abbildung 25: Häufigkeit des Schreibens von Textnachrichten bzw. der Nutzung der Spracheingabe während des Fahrens, Fragebogendaten $(N=82)$.

Aus einer präzisierenden Frage zur Nutzungshäufigkeit in Relation zur Fahrzeit lässt sich bestätigen, dass alle Teilnehmer eine generelle Bereitschaft zum Bearbeiten von Textnachrichten aufwiesen, wobei Lesen häufiger als Schreiben berichtet wurde (siehe 
Abbildung 26). Entsprechend kann davon ausgegangen werden, dass die Teilnehmer der Untersuchung grundsätzlich geübt darin waren während des Fahrens Textnachrichten zu lesen und zu schreiben.

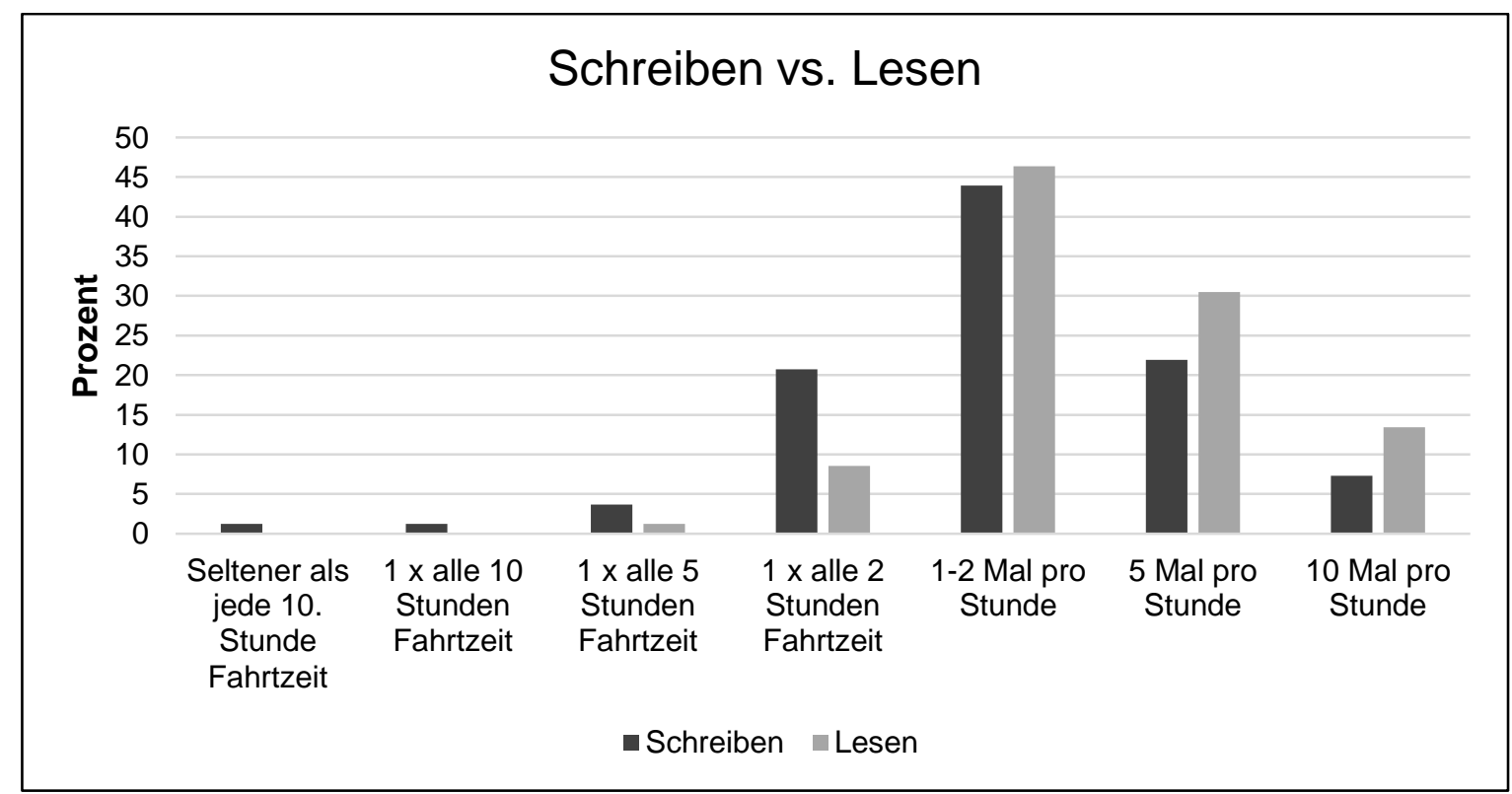

Abbildung 26: Häufigkeit des Schreibens von Textnachrichten bzw. der Nutzung der Spracheingabe während des Fahrens, Fragebogendaten $(N=82)$.

\subsubsection{Analyse von überdauernden (Persönlichkeits-)eigenschaften bezüglich des Textens während des Fahrens}

Die Einstellungen bzw. Persönlichkeitseigenschaften wurden ursprünglich erhoben, um sie zur Bereitschaft zum Bearbeiten von Textnachrichten innerhalb der Fahrsimulatorstudie in Beziehung $\mathrm{zu}$ setzen. Allerdings zeigte sich in der Fahrsimulatorstudie, dass ein Großteil der Teilnehmer praktisch alle Nebenaufgaben bearbeitete (Kapitel 5.2.3). Daher ist eine solche Analyse wenig sinnvoll, da keine Varianz in den Daten besteht und mögliche Zusammenhänge durch Deckeneffekte überlagert werden. Stattdessen werden die erfassten Einstellungen bzw. Persönlichkeitseigenschaften an dieser Stelle lediglich zur Charakterisierung der Stichprobe verwendet.

Zur Erfassung der Einstellung gegenüber dem Schreiben von Textnachrichten sollten die Teilnehmer auf einer 7-stufigen Zustimmungsskala für insgesamt fünf verschiedene Aussagen angeben, wie sie dem Schreiben von Textnachrichten beim Autofahren gegenüberstehen (Kapitel 5.1.2). Sie waren dem Schreiben von Textnachrichten während des Fahrens gegenüber mehrheitlich positiv eingestellt $(M=5,7 ; S D=0,7$, siehe Abbildung 27 links). Dies ist nicht überraschend, da die Stichprobe bewusst aus Teilnehmern zusammengestellt wurde, die dieses Verhalten grundsätzlich zeigen.

Ferner wurde auf einer 7-stufigen Zustimmungsskala erfasst, wie stark die Teilnehmer eine Textnachricht aus Gewohnheit schreiben. In Abbildung 27 rechts ist die Zustimmung über die Teilnehmer hinweg dargestellt. Die Teilnehmer zeigten mehrheitlich die Tendenz, Textnachrichten einfach aus Gewohnheit zu schreiben $(M=3,7 ; S D=1,1)$. 


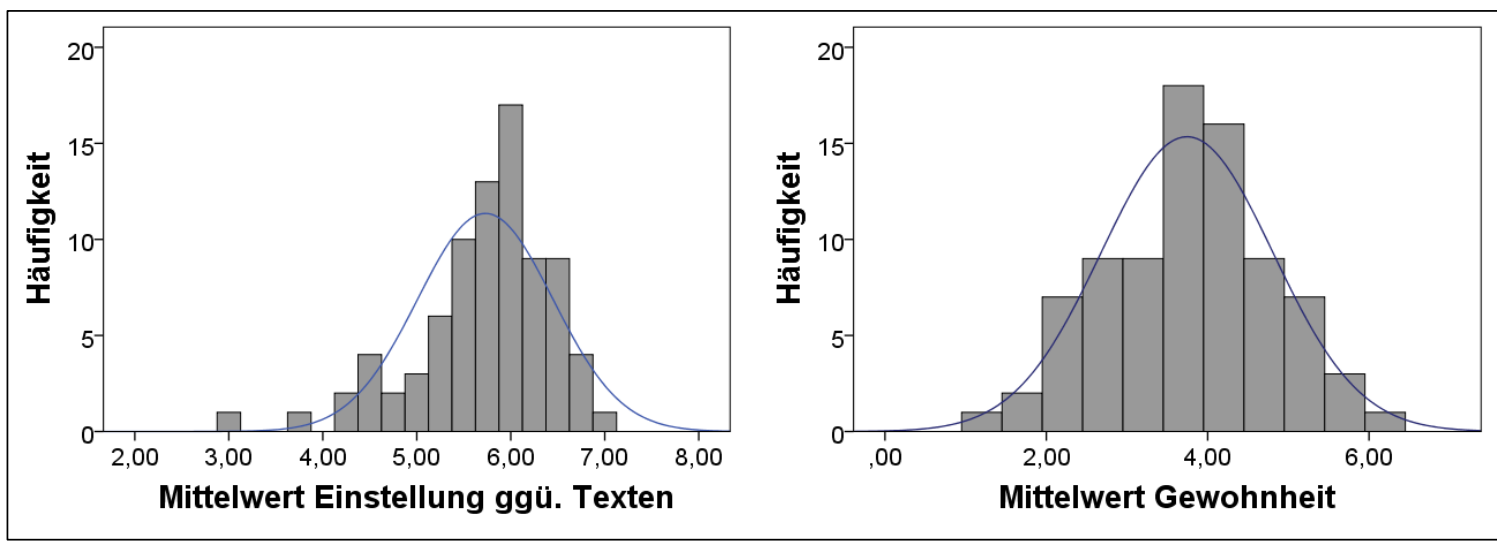

Abbildung 27: Häufigkeit der Mittelwerte der Teilnehmer auf der Skala zur Einstellung gegenüber dem Schreiben von Textnachrichten während des Fahrens (links) und auf der zum gewohnheitsmäßigen Schreiben von Textnachrichten, Fragebogendaten (rechts, $N=82$ Personen).

Bezüglich der einzelnen Komponenten der Theorie des geplanten Verhaltens ist erkennbar dass die Teilnehmer, leicht abweichend zum Ergebnis der reinen Einstellungsskala (S. 64), gegenüber dem Schreiben von Textnachrichten während des Fahrens im Schnitt neutral eingestellt waren (siehe Abbildung 28). Der Mittelwert für die Kontrolle darüber Textnachrichten während des Fahrens zu schreiben lag über dem Skalenmittel. Das heißt, dass die Teilnehmer der Ansicht waren, die Kontrolle über das Verhalten zu haben. Die Soziale Norm gliedert sich nochmals in die wahrgenommene Norm, moralische Norm und Gruppennormen, welche auch getrennt ausgewertet wurden. Für die Skala zur Gruppennorm lag der Durchschnittswert nah am Skalenmittelpunkt, was darauf hindeutet, dass die Teilnehmer der Meinung waren, dass ihre Freunde das Schreiben von Textnachrichten während des Fahrens weder besonders positiv noch negativ fanden. Auch bezogen auf ihre eigene moralische Norm, fanden sie es weder besonders unmoralisch noch moralisch unbedenklich Textnachrichten zu schreiben. Für die wahrgenommene Norm zeigt sich, dass die Teilnehmer bei Personen die sie respektieren das Gefühl hatten, dass diese dem Schreiben von Textnachrichten während des Fahrens eher neutral gegenüberständen.

Ebenfalls sollten die Teilnehmer ihren eigenen Fahrstil auf den Dimensionen Vorsicht und Routine einschätzen. Die Teilnehmer bewerteten sich auf einer 7-stufigen Skala als eher routinierte Fahrer $(M=5,4, S D=0,7)$ und als durchschnittlich vorsichtig $(M=3,2$, $S D=0,7)$.

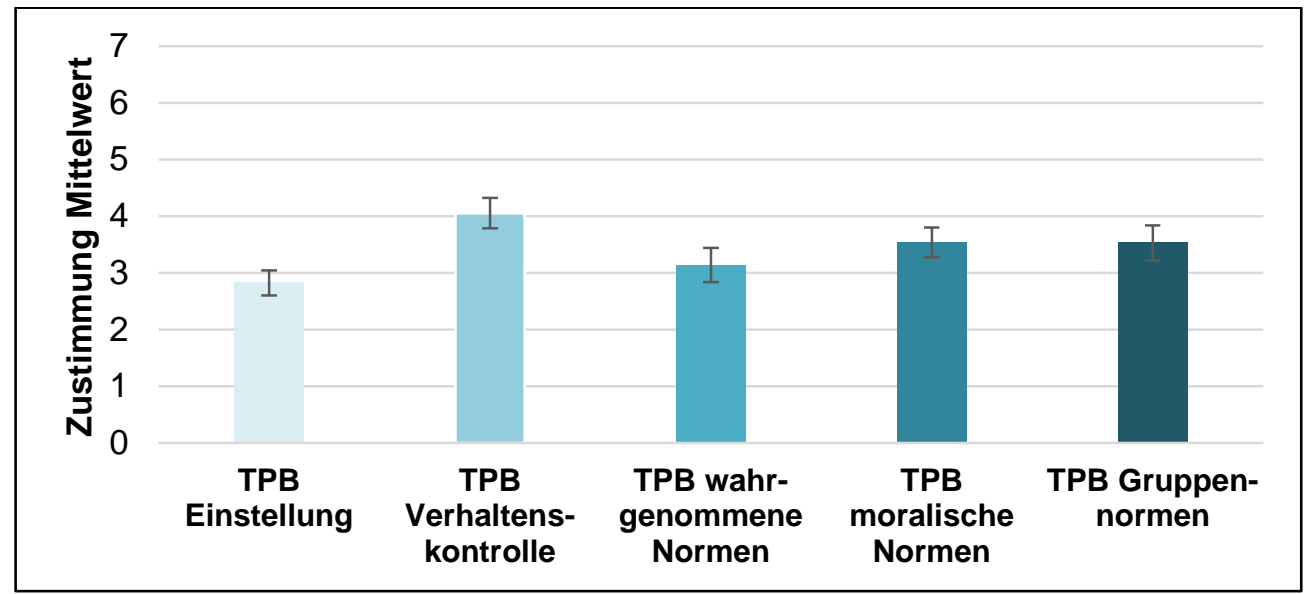

Abbildung 28: Mittelwerte der Zustimmung für die Skalen der Theorie des geplanten Verhaltens bezogen auf das Schreiben von Textnachrichten während des Fahrens, Fragebogendaten ( $N=82$ Personen). 


\subsubsection{Bearbeitung der Nebentätigkeit während der Fahrt im Simulator}

Die Bearbeitung der Nebentätigkeit wurde, über alle Teilnehmer hinweg, insgesamt $1.640 \mathrm{Mal}$ angetriggert. Nur in 45 Fällen wurde die Aufgabe durch die Teilnehmer nicht bearbeitet $(2,7 \%)$. Am häufigsten wurde die Nebentätigkeit bei der Spracheingabe (15 Fälle) und beim Lesen am Mobiltelefon (14 Fälle) nicht bearbeitet. Mit sechs Fällen wurde die Bearbeitung am seltensten beim manuellen Schreiben abgelehnt, während dies beim Vorlesen in 10 Fällen geschah.

Von den 65 Teilnehmern, die während der Untersuchung Nebentätigkeiten ausführten, ließen 18 Teilnehmer mindestens eine Möglichkeit zur Bearbeitung aus (Spannweite zwischen 1 und 6 ausgelassenen Fälle). Es zeigt sich, dass vor allem in Situationen mit Kurven die Bearbeitung von Nebentätigkeiten unterlassen wurde (27 Fälle). Ebenfalls wurden mehrfach die Aufgaben nicht bearbeitet, wenn parkende Fahrzeuge an einem der beiden Fahrbahnränder standen (11 Fälle) oder sich die Fahrer in einem Streckenabschnitt mit 30 km/h Geschwindigkeitsbegrenzung befanden (7 Fälle).

Die Dauer der verschiedenen Nebentätigkeiten unterschied sich erwartungsgemäß signifikant zwischen den einzelnen Nebentätigkeiten $(F(3,126)=118,95 ; p<, 001$; $\eta_{p}^{2}=, 739$, siehe Abbildung 29). Für das Schreiben einer Textnachricht am Mobiltelefon wurde deutlich mehr Zeit benötigt als für die anderen Tätigkeiten, während die Spracheingabe die geringste Zeit beanspruchte. Paarweise Vergleiche ergaben signifikante Unterschiede zwischen allen einzelnen Nebentätigkeiten (alle $p \leq .001$ ).

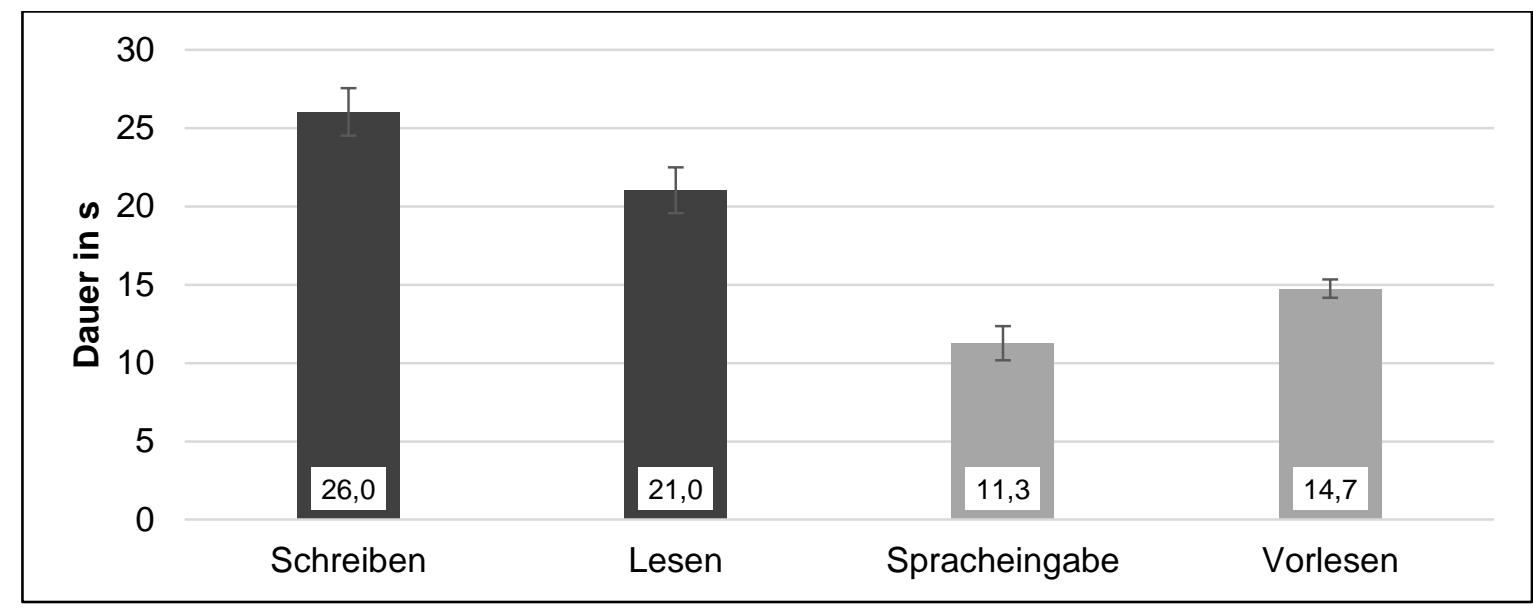

Abbildung 29: Dauer der Bearbeitung der Nebentätigkeit, Videodaten $(N=82)$.

Außerdem wurden für die Nebentätigkeiten Schreiben und Spracheingabe die Länge der verfassten Nachrichten verglichen (gemessen als Anzahl der verwendeten Zeichen, inklusive Leerzeichen). Die manuell eingegeben Nachrichten waren deutlich kürzer als die per Spracheingabe verfassten (siehe Tabelle 19). Zudem waren mehr Fehler in den manuell eingegebenen Nachrichten zu beobachten, z.B. „Das Fach war Mathe“ oder „Fußball“, im Vergleich zu Spracheingabe, z.B. „Mein liebstes Fach war Deutsch aber Sport war auch nicht schlecht".

Tabelle 19: Anzahl bearbeiteter Zeichen pro Textnachricht, Videodaten.

\begin{tabular}{|l|c|c|c|c|}
\hline Bedingung & M & SD & Min & Max \\
\hline Schreiben manuell & 17,7 & 10,2 & 2 & 64 \\
\hline Spracheingabe & 81,5 & 40,5 & 5 & 216 \\
\hline
\end{tabular}


Ferner wurden die Bedienstrategien beim Lesen und Schreiben einer Textnachricht durch manuelle Eingabe analysiert. Beim Lesen hielten alle Teilnehmer das Mobiltelefon in der rechten Hand und tippten mit dem rechten Daumen. Dabei hielten es die meisten vor die rechte Lenkradseite oder rechts oben vor das Lenkrad (siehe Tabelle 20).

Tabelle 20: Bedienstrategien beim Lesen - Position des Mobiltelefons im Fahrzeug, Videodaten $(n=33)$.

\begin{tabular}{|l|c|c|}
\hline Position & N & Anteil \% \\
\hline vor rechter Lenkradseite & 16 & 48,5 \\
\hline rechts oben vor Lenkrad & 8 & 24,2 \\
\hline mittig vor Lenkrad / auf Lenkradkreuz & 4 & 12,1 \\
\hline rechts unten vor Lenkrad & 3 & 9,1 \\
\hline Sonstiges & 2 & 6,0 \\
\hline
\end{tabular}

Beim manuellen Schreiben wurde auch von fast allen Teilnehmern (94\%) der rechte Daumen genutzt, lediglich 6\% nahmen beide Daumen. Ein Drittel der Teilnehmer nutzten die Wortvervollständigungsfunktion (33\%). Auch zum Schreiben wurde das Mobiltelefon meist vor der rechten Lenkradseite positioniert, in einigen Fällen auch rechts oben bzw. unten vor dem Lenkrad (siehe Tabelle 21).

Tabelle 21: Bedienstrategien beim Schreiben - Position des Handys im Fahrzeug, Videodaten $(n=33)$.

\begin{tabular}{|l|c|c|}
\hline Position & N & Anteil \% \\
\hline vor rechter Lenkradseite & 15 & 45,5 \\
\hline rechts oben vor Lenkrad & 6 & 18,2 \\
\hline rechts unten vor Lenkrad & 5 & 15,2 \\
\hline mittig vor Lenkrad / auf Lenkradkreuz & 3 & 9,1 \\
\hline rechts neben Lenkrad & 3 & 9,1 \\
\hline (unten) Höhe Oberschenkel & 1 & 3,0 \\
\hline
\end{tabular}




\subsubsection{Vergleich Fahrperformanz auf geraden Streckenabschnitten mit und ohne Nebentätigkeit}

Für den Vergleich der Fahrperformanz auf geraden Streckenabschnitten mit und ohne Nebentätigkeit wurden für jeden Teilnehmer Streckenabschnitte individueller Länge betrachtet. In Tabelle 22 sind die durchschnittliche Länge und die Fahrzeit der analysierten geraden Streckenabschnitte für die beiden Geschwindigkeitsbegrenzungen $30 \mathrm{~km} / \mathrm{h}$ und $50 \mathrm{~km} / \mathrm{h}$ abgetragen. Variationen ergeben sich aus unterschiedlichen Bearbeitungsdauern für die Nebentätigkeit, unterschiedlich schneller Aufnahme der Nebentätigkeit, sowie Unterschieden in den gefahrenen Geschwindigkeiten. So sind z.B. die analysierten Streckenabschnitte für die Spracheingabe etwas kürzer (und die damit verbundene Fahrzeit geringer) als bei den anderen Nebentätigkeiten, was in der generell kürzeren Bearbeitungsdauer bei Spracheingabe begründet ist (siehe S. 66).

Tabelle 22: Durchschnittliche Dauer und Länge der analysierten geraden Streckenabschnitte, Videodaten $(n=65)$.

\begin{tabular}{|c|c|c|c|c|}
\hline \multirow{2}{*}{$\begin{array}{l}\text { Art der } \\
\text { Nebentätigkeit }\end{array}$} & \multicolumn{2}{|c|}{ Streckenabschnitt $30 \mathrm{~km} / \mathrm{h}$} & \multicolumn{2}{|c|}{ Streckenabschnitt $50 \mathrm{~km} / \mathrm{h}$} \\
\hline & $\varnothing$ Dauer in $\mathbf{s}$ & $\varnothing$ Länge in $\mathbf{m}$ & $\varnothing$ Dauer in s & $\varnothing$ Länge in $\mathrm{m}$ \\
\hline Schreiben & 12,2 & 118,8 & 14,1 & 216,7 \\
\hline Lesen & 13,7 & 132,3 & 14,2 & 213,7 \\
\hline Spracheingabe & 10,0 & 90,7 & 10,4 & 157,2 \\
\hline Vorlesen & 13,3 & 123,4 & 13,5 & 199,3 \\
\hline Gesamt & 12,3 & 116,9 & 13,1 & 197,6 \\
\hline
\end{tabular}

Vergleich der Geschwindigkeit mit und ohne Nebentätigkeit auf gerader Strecke

Für den Vergleich der Geschwindigkeiten mit und ohne Nebentätigkeit wurden von 65 Teilnehmern insgesamt 127 Fahrten analysiert. Es wurden für eine Fahrt jeweils ein Streckenabschnitt mit und ein Streckenabschnitt ohne Nebenaufgabe untersucht. In Abbildung 30 ist die Durchschnittsgeschwindigkeit für die verschiedenen Nebentätigkeiten bei einer Geschwindigkeitsbegrenzung von $30 \mathrm{~km} / \mathrm{h}$ dargestellt. Es zeigt sich, dass Teilnehmer insbesondere beim Schreiben einer Textnachricht langsamer fuhren als beim Befahren eines vergleichbaren Streckenabschnitts (bei der gleichen Fahrt) ohne Eingabe einer Textnachricht. Die Varianzanalyse bestätigt, dass auf dem geraden Streckenabschnitt mit Nebentätigkeit signifikant langsamer gefahren wurde als auf dem Streckenabschnitt ohne Nebentätigkeit $\left(F(1,123)=6,26 ; p=, 014 ; \eta^{2}{ }_{p}=, 048\right)$. Es zeigte sich keine Interaktion zwischen der Art der Nebentätigkeit und deren Bearbeitung $\left(F(3,123)=1,87 ; p=, 138 ; \eta_{p}^{2}=, 044\right)$. Allerdings zeigte sich ein signifikanter Unterschied zwischen den verschiedenen Arten der Nebentätigkeiten $(F(3,123)=4,65$; $\left.p=, 004 ; \eta_{p}^{2}=, 102\right)$. Die Teilnehmer, die während ihrer Fahrt manuell Nachrichten schrieben, waren generell, sowohl mit als auch ohne Nebenaufgabe, mit signifikant höheren Geschwindigkeiten unterwegs als wenn sie die Spracheingabe $(p=, 005)$ oder Vorlesefunktion nutzten $(p=, 047)$. Darüber hinaus gab es noch eine Teilnehmergruppe, die die gesamte Strecke ohne Nebenaufgaben fuhr. Diese Baselinegruppe wurde dabei nicht in die Berechnung einbezogen, sondern nur zum visuellen Vergleich in der Abbildung 30 dargestellt. Es zeigt sich aber, dass die Teilnehmer die nie einer Nebentätigkeit nachgingen, schneller waren als die Teilnehmer die gerade einer Nebentätigkeit, egal welcher Art, nachgingen. Dies könnte darauf hindeuten, dass die Teilnehmer die Bearbeitung einer Nebentätigkeit durch die Verringerung der Geschwindigkeit bei der Bearbeitung kompensierten. Außerdem waren sie auf 
Streckenabschnitten auf denen alle Teilnehmer keine Nebentätigkeit ausführten schneller als die Teilnehmer der Nebenaufgabenbedingungen (außer beim Schreiben).

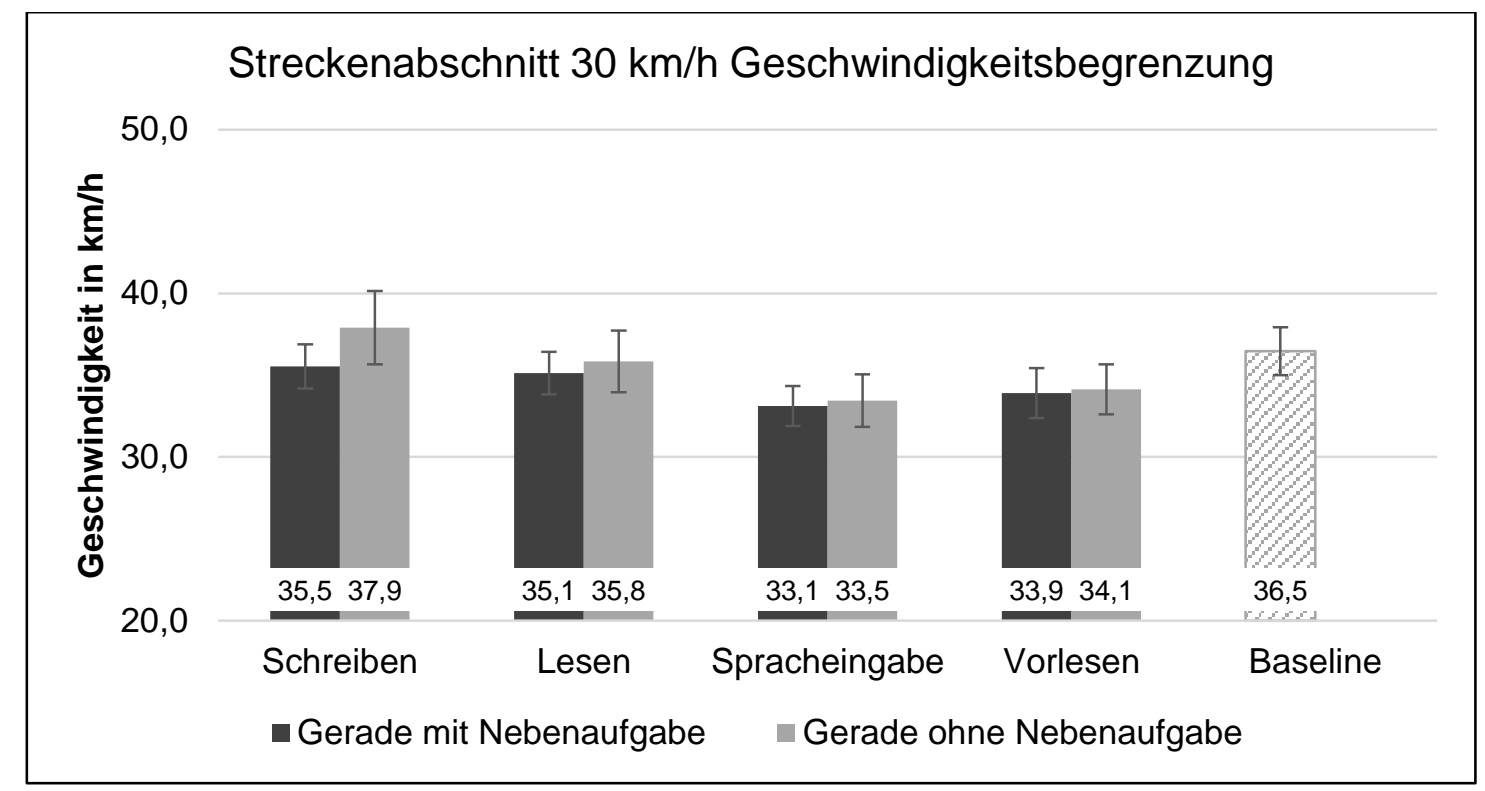

Abbildung 30: Durchschnittliche Geschwindigkeit Streckenabschnitt 30 km/h Geschwindigkeitsbegrenzung, Fahrdaten ( $N=127$ Fahrten, 65 Teilnehmer, Fehlerbalken 95\% Konfidenzintervall).

Für die Analyse der geraden Abschnitte bei Geschwindigkeitsbegrenzung von $50 \mathrm{~km} / \mathrm{h}$ fand sich kein signifikanter Unterschied zwischen den Abschnitten mit und ohne Nebentätigkeit $\left(F(1,123)=0,75 ; p=, 389 ; \eta_{p}^{2}=, 006\right.$, siehe Abbildung 31). Es bestand ebenfalls keine Interaktion zwischen der Bearbeitung und der Art der Nebentätigkeit $\left(F(3,123)=0,91 ; \quad p=, 437 ; \quad \eta_{p}^{2}=, 022\right)$. Auch die genauere Betrachtung der Nebentätigkeiten ergab keinen signifikanten Unterschied bezogen auf die Geschwindigkeit $\left(F(3,123)=1,23 ; p=, 301 ; \eta_{p}^{2}=, 029\right)$. Zieht man allerdings die Baselinebedingung zum rein deskriptiven Vergleich hinzu, so scheint es, dass in den Fahrten mit Nebentätigkeit generell geringere Geschwindigkeiten erreicht wurden, unabhängig davon, ob die Teilnehmer zum jeweiligen Zeitpunkt tatsächlich eine Aufgabe bearbeiteten.

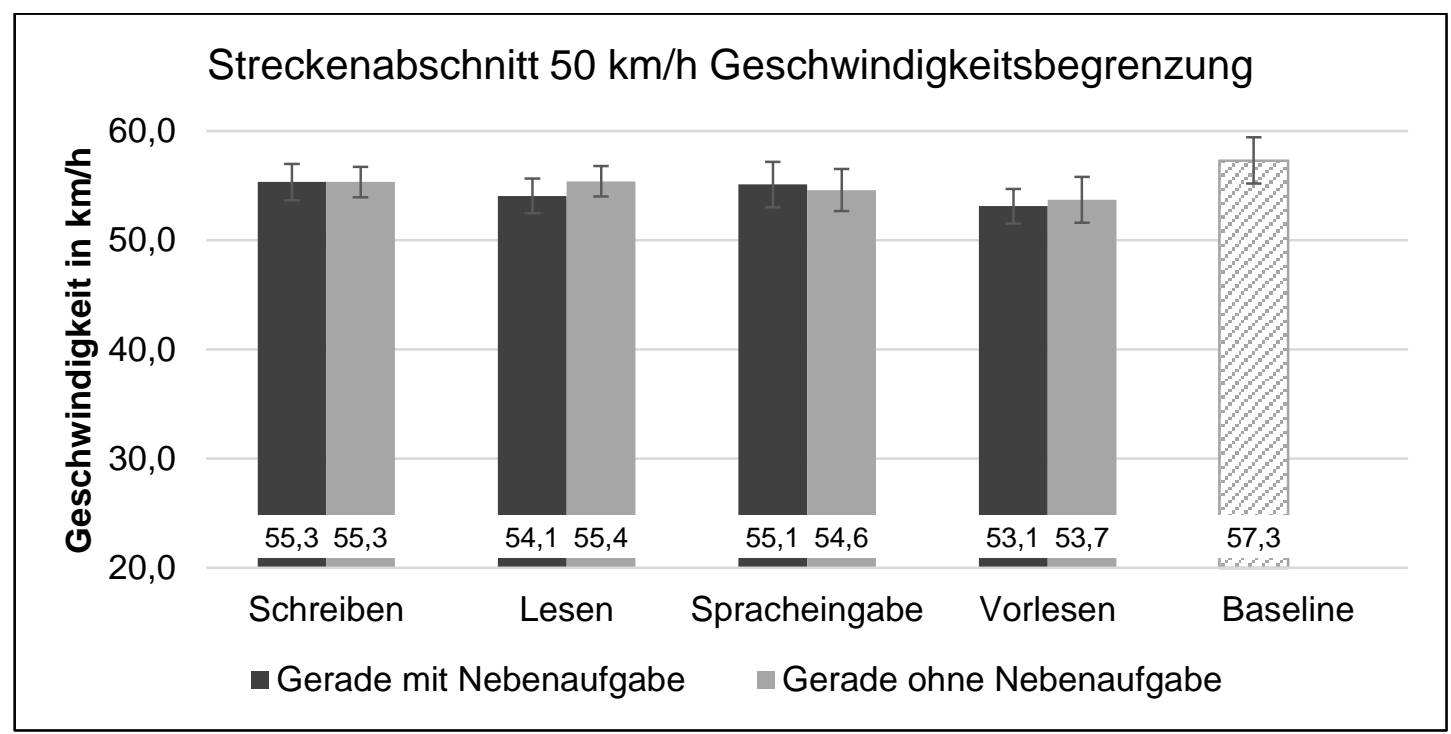

Abbildung 31: Durchschnittliche Geschwindigkeit Streckenabschnitt 50 km/h Geschwindigkeitsbegrenzung, Fahrdaten ( $N=127$ Fahrten, 65 Teilnehmer, Fehlerbalken 95\% Konfidenzintervall). 
Zusätzlich zur Geschwindigkeit wurde auch die Standardabweichung der lateralen Spurposition für das Fahren mit und ohne Nebentätigkeit verglichen. Generell muss man hierbei beachten, dass es sich bei diesen Abweichungen jeweils um wenige $\mathrm{cm}$ handelt. In Abbildung 32 ist erkennbar, dass ohne Nebenaufgabe signifikant stärkere Schwankungen in der Spurposition zu verzeichnen waren als mit Nebenaufgabe $\left(F(1,123)=5,72 ; p=, 018 ; \eta_{p}^{2}=, 044\right)$. Ebenfalls wurde ein signifikanter Haupteffekt für die Art der Nebentätigkeit gefunden $\left(F(3,123)=3,54 ; p=, 017 ; \eta_{p}^{2}=, 080\right)$. Die post-hoc Tests ergaben, dass sich Lesen signifikant von der Spracheingabe unterschied $(p=, 013)$, mit deutlich geringeren Schwankungen in der Spurposition bei der Spracheingabe. Eine signifikante Interaktion zwischen der Bearbeitung und der Art der Nebentätigkeit konnte allerdings nicht festgestellt werden $(F(3,123)=0,19 ; p=, 905$; $\left.\eta_{p}^{2}=, 005\right)$.

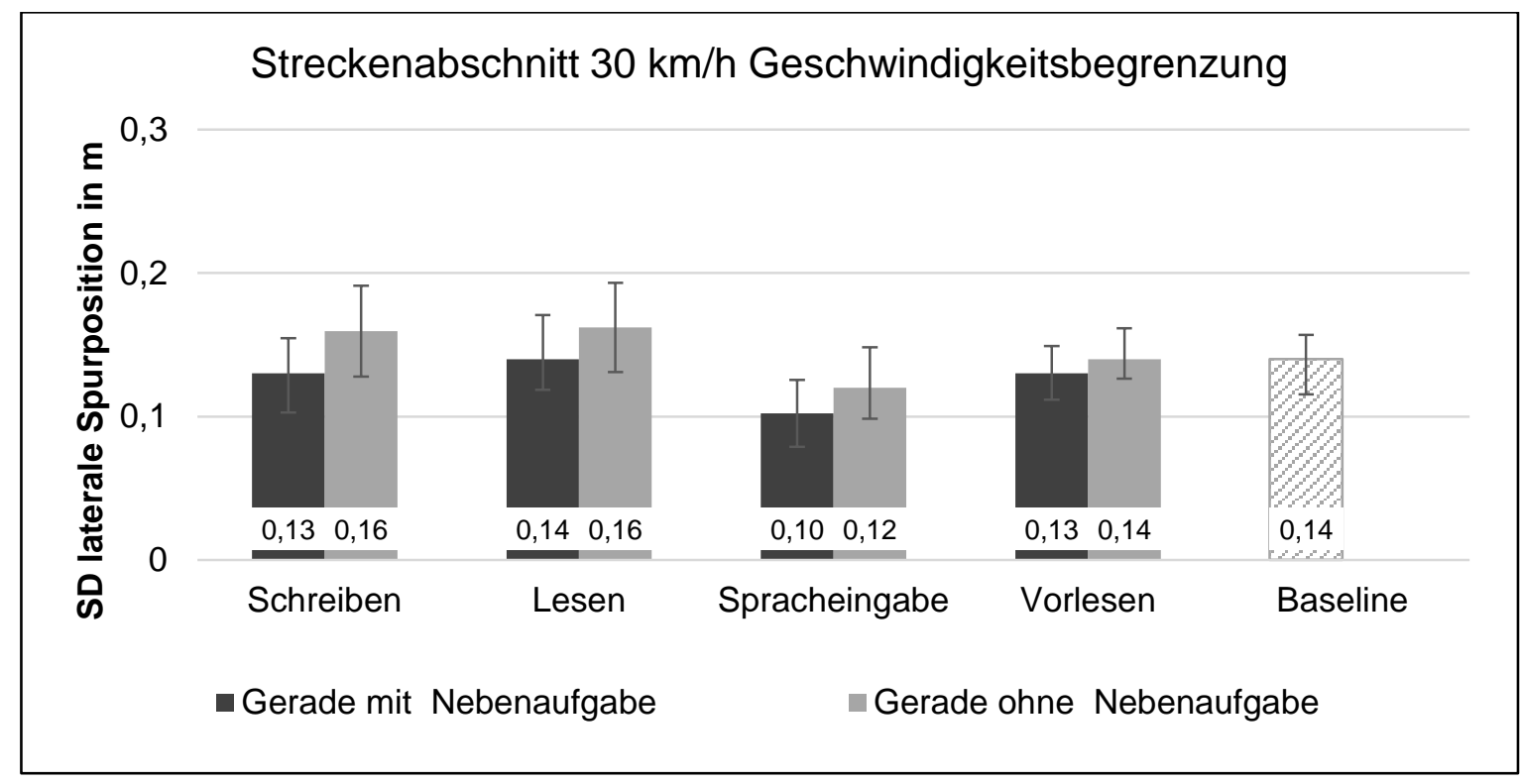

Abbildung 32: Standardabweichung der lateralen Spurposition für Streckenabschnitt 30 km/h Geschwindigkeitsbegrenzung, Fahrdaten ( $N=127$ Fahrten, 65 Teilnehmer, Fehlerbalken 95\% Konfidenzintervall).

Auf geraden Strecken mit $50 \mathrm{~km} / \mathrm{h}$ Geschwindigkeitsbegrenzung wurden keine signifikanten Unterschiede in der Standardabweichung der Spurposition zwischen dem Fahren mit und ohne Nebentätigkeit gefunden $\left(F(1,122)=2,55 ; p=, 113 ; \eta_{p}^{2}=, 020\right.$, siehe Abbildung 33). Ebenso unterschieden sich die Nebentätigkeiten nicht signifikant voneinander $\left(F(3,122)=0,47 ; \quad p=, 704 ; \quad \eta_{p}^{2}=, 011\right)$. Allerdings ergab sich eine signifikante Interaktion zwischen der Nebenaufgabe und dem Fahren mit und ohne Nebentätigkeit $\left(F(3,122)=5,52 ; p=, 001 ; \eta_{p}^{2}=, 119\right)$. In der Gruppe der Spracheingabe zeigten sich beim Fahren ohne Nebentätigkeit starke Schwankungen in der Spurposition. 


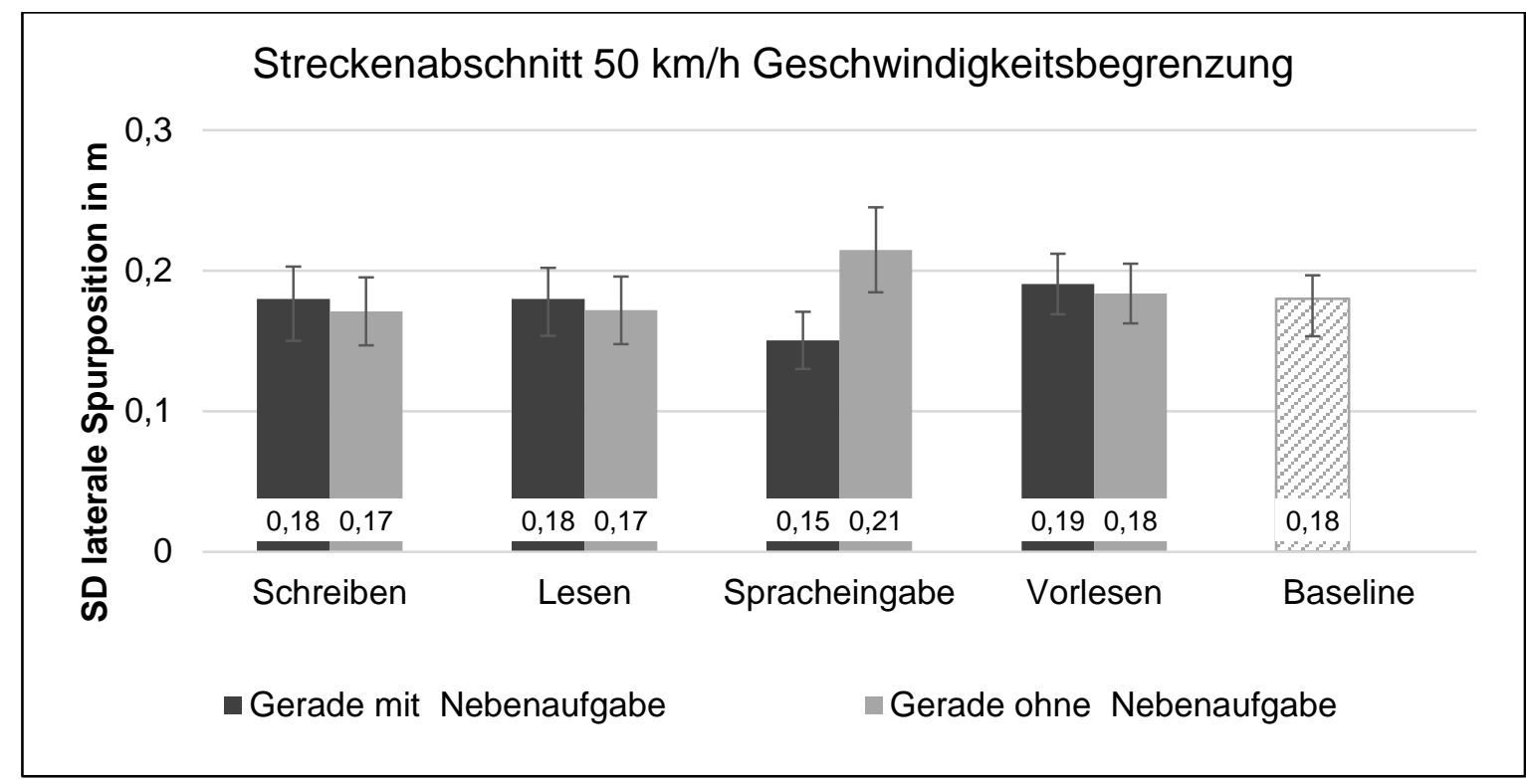

Abbildung 33: Standardabweichung laterale Distanz für Streckenabschnitt 50 km/h Geschwindigkeitsbegrenzung, Fahrdaten ( $N=127$ Fahrten, 65 Teilnehmer, Fehlerbalken 95\% Konfidenzintervall). 


\subsubsection{Besondere Verkehrssituationen - Kind auf der Straße}

\section{Unfälle und Beinaheunfälle}

Bei der Fahrbahnüberquerung des Kindes gab es im Durchschnitt bei $12,4 \%$ der Situationen einen Zusammenstoß zwischen Egofahrzeug und Kind, sowie in 24,8\% einen Beinaheunfall. Mit dem Ball als Vorinformation wurden weniger Zusammenstöße und Beinaheunfälle registriert als ohne Vorinformation (siehe Tabelle 23). Die Fahrer scheinen durch den Ball bereits darauf vorbereitet zu sein zu reagieren. Hinsichtlich der verschiedenen Nebentätigkeiten wurden die meisten Unfälle und Beinaheunfälle beim manuellen Schreiben einer Nachricht am Mobiltelefon beobachtet, häufiger ohne Vorinformation als mit Vorinformation. Am seltensten kam es bei der Spracheingabe zu Unfällen, egal ob mit oder ohne Vorinformation.

Tabelle 23: Anzahl und prozentuale Anteile der Unfälle und Beinaheunfälle mit und ohne Vorinformation bei kritischer Situation Kind, Videodaten.

\begin{tabular}{|c|c|c|c|c|c|c|c|c|c|c|c|c|}
\hline & \multicolumn{2}{|c|}{ Schreiben } & \multicolumn{2}{|c|}{ Lesen } & \multicolumn{2}{|c|}{$\begin{array}{l}\text { Sprach- } \\
\text { eingabe }\end{array}$} & \multicolumn{2}{|c|}{ Vorlesen } & \multicolumn{2}{|c|}{ Baseline } & \multicolumn{2}{|c|}{ Gesamt } \\
\hline & $N$ & $\%$ & $N$ & $\%$ & $N$ & $\%$ & $N$ & $\%$ & $N$ & $\%$ & $N$ & $\%$ \\
\hline \multicolumn{13}{|c|}{ Mit Vorinformation } \\
\hline Unfall & 2 & 14,3 & 2 & 12,5 & 0 & 0,0 & 0 & 0,0 & 2 & 11,8 & 6 & 7,8 \\
\hline \begin{tabular}{|l|} 
Beinahe- \\
unfall
\end{tabular} & 5 & 35,7 & 3 & 18,8 & 1 & 6,7 & 2 & 13,3 & 3 & 17,6 & 14 & 18,2 \\
\hline $\begin{array}{l}\text { keine } \\
\text { Gefahr }\end{array}$ & 7 & 50,0 & 11 & 68,8 & 14 & 93,3 & 13 & 86,7 & 12 & 70,6 & 57 & 74,0 \\
\hline Gesamt & 14 & 100,0 & 16 & 100,0 & 15 & 100,0 & 15 & 100,0 & 17 & 100,0 & 77 & 100,0 \\
\hline \multicolumn{13}{|c|}{ Ohne Vorinformation } \\
\hline Unfall & 4 & 25,0 & 2 & 14,3 & 1 & 7,7 & 3 & 18,8 & 3 & 17,6 & 13 & 17,1 \\
\hline $\begin{array}{l}\text { Beinahe- } \\
\text { unfall }\end{array}$ & 6 & 37,5 & 5 & 35,7 & 4 & 30,8 & 3 & 18,8 & 6 & 35,3 & 24 & 31,6 \\
\hline $\begin{array}{l}\text { keine } \\
\text { Gefahr }\end{array}$ & 6 & 37,5 & 7 & 50,0 & 8 & 61,5 & 10 & 62,5 & 8 & 47,1 & 39 & 51,3 \\
\hline Gesamt & 16 & 100,0 & 14 & 100,0 & 13 & 100,0 & 16 & 100,0 & 17 & 100,0 & 76 & 100,0 \\
\hline
\end{tabular}

\section{Reduktion Gaspedaldruck}

Als Reaktion auf das Kind bzw. die Vorinformation wurde erwartet, dass die Teilnehmer vom Gas gehen, und letztlich bremsen. Entsprechend wurde zunächst betrachtet, wann eine Reduktion des Gaspedaldrucks um mindestens 30\% erfolgt. Analysiert wurden die vergangene Zeit seit Sichtbarkeit der Vorinformation (bzw. im Szenario ohne Vorinformation das Passieren des entsprechenden Streckenpunktes), der Abstand zum Kind, sowie die Time to Collision in Bezug auf das Kind (siehe Tabelle 24, S. 75).

Wie erwartet reduzierten die Teilnehmer signifikant früher den Gaspedaldruck, wenn sie durch den Ball vorgewarnt worden waren $(U=1.241,50 ; p<, 001)$. Generell fand sich ein signifikanter Unterschied zwischen den Nebentätigkeiten $(H(4)=10,25 ; p=, 036)$. Bei den post-hoc Vergleichen unterschieden sich die einzelnen Bedingungen allerdings nicht signifikant voneinander (kleinstes $p=, 115$ ). 
Analog erfolgte die Reduktion des Gaspedaldrucks mit dem Ball als Vorinformation in signifikant größerem Abstand zum Kind als ohne Vorinformation $(U=1.347,0 ; p<, 001$, siehe Tabelle 24, S. 75). Erneut wurde auch ein signifikanter Unterschied zwischen den Nebentätigkeiten gefunden $(H(4)=9,61 ; p=, 048)$. Allerdings ergaben die post-hoc Tests keine signifikanten Unterschiede zwischen den einzelnen Nebentätigkeiten bzw. der Baseline (kleinstes $p=, 097$ ).

Ähnliche Ergebnisse ergaben sich auch bei der Analyse der Time to Collision bei Reduktion des Gaspedaldrucks, die ohne Ball als Vorinformation signifikant geringer ausfiel mit Vorinformation $(U=1.613,0 ; p<, 001$, siehe Tabelle 24, S. 75). Zwischen den Nebentätigkeiten bestand ein signifikanter Unterschied $(H(4)=11,60 ; p=, 021)$. Die pochoc Vergleiche ergaben, dass bei Teilnehmern, die manuell eine Textnachricht am Mobiltelefon verfassten, die Reduktion des Gaspedaldrucks bei signifikant geringerer Time to Collision erfolgte als bei denen, die sich eine Nachricht vorlesen ließen $(p=, 034)$. Zwischen den anderen Nebentätigkeiten bzw. im Vergleich zur Baseline bestanden keine signifikanten Unterschiede.

\section{Bremspedalbetätigung}

Für die Betätigung des Bremspedals wurden, analog zur Reduktion des Gaspedaldrucks, der Zeitpunkt (in Relation zum Passieren des Streckenpunktes, an dem die Vorinformation sichtbar wurde bzw. sichtbar geworden wäre) der Abstand sowie die Time to Collision zum Kind bei erstmaliger Betätigung des Bremspedals analysiert (siehe Tabelle 24, S. 75).

In Abbildung 34 ist die Zeit bis zur ersten Betätigung des Bremspedals dargestellt. Es ist erkennbar, dass die Teilnehmer das Bremspedal zeitiger betätigten, wenn sie durch den Ball vorgewarnt wurden. Dieser Unterschied für die Vorinformation war signifikant $\left(F(1,138)=63,37 ; p<, 001 ; \eta_{p}^{2}=, 315\right.$. Zwischen den verschiedenen Nebentätigkeiten zeigte sich kein signifikanter Unterschied $\left(F(4,138)=1,85 ; p=, 122 ; \eta^{2}{ }_{p}=, 051\right)$. Es wurde in allen Bedingungen ähnlich schnell reagiert. Auch gab es keine signifikante Interaktion zwischen Vorinformation und Nebentätigkeit $(F(4,138)=0,99 ; p=, 415$; $\left.\eta_{p}^{2}=, 028\right)$. Das bedeutet, dass die Vorinformation bei allen Nebentätigkeiten in vergleichbarer Weise funktionierte.

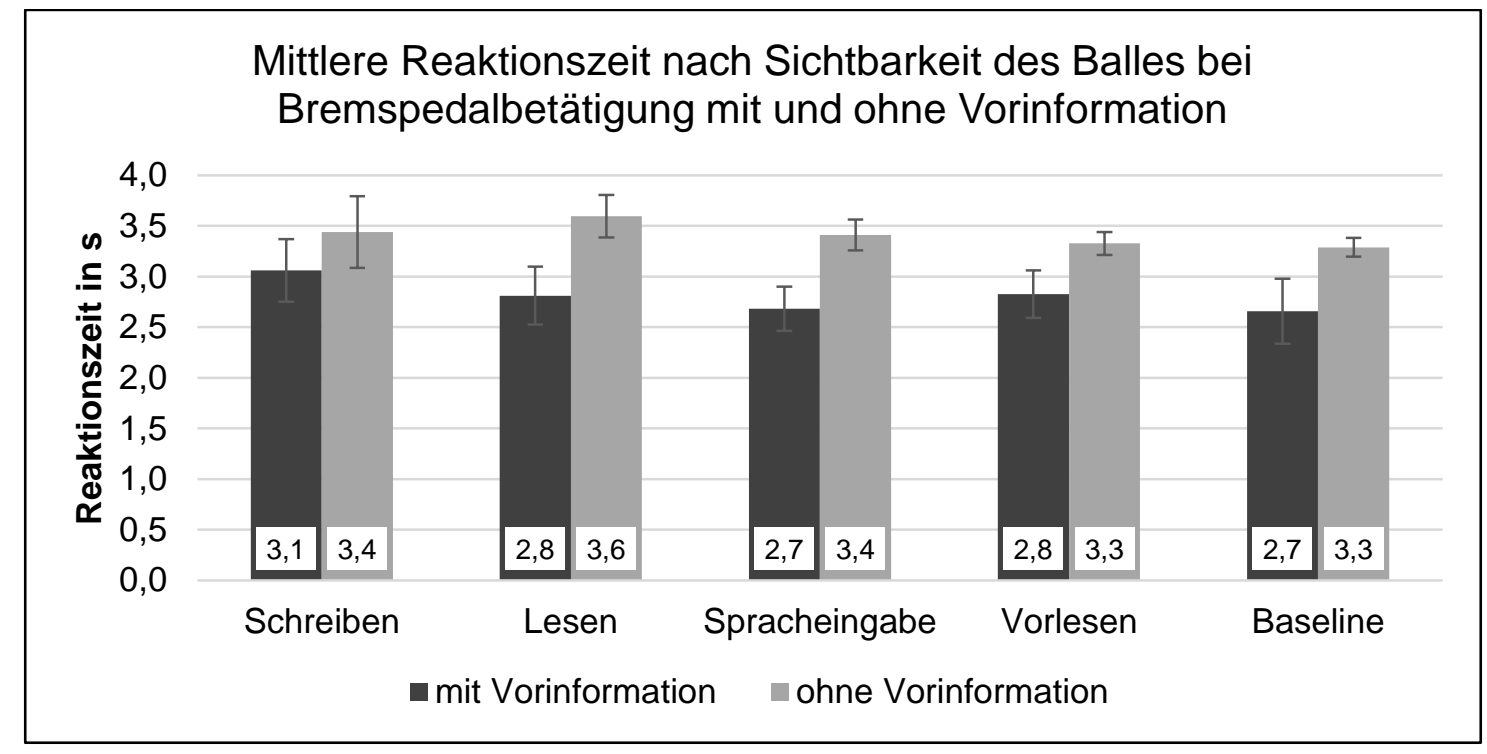

Abbildung 34: Reaktionszeit nach Sichtbarkeit des Balles bei Bremspedalbetätigung mit und ohne Vorinformation, Fahrdaten ( $N=148$ Situationen, Fehlerbalken 95\% Konfidenzintervall). 
Auch für den Abstand zum Kind bei Bremspedalbetätigung mit und ohne Vorinformation ergab sich ein signifikanter Unterschied $\left(F(1,138)=34,47 ; p<, 001 ; \eta_{p}^{2}=, 200\right)$, mit größerem Abstand bei Vorinformation (siehe Abbildung 35). Zwischen den einzelnen Nebentätigkeiten und auch der Baseline bestand kein Unterschied $(F(4,138)=1,51$; $\left.p=, 203 ; \eta_{p}^{2}=, 042\right)$. Betrachtet man die Daten auf deskriptiver Ebene, so scheinen die Teilnehmer beim Schreiben der Textnachricht am wenigsten von der Vorinformation profitiert zu haben als in den anderen Bedingungen, jedoch ergab sich keine signifikante Interaktion zwischen Vorinformation und Nebentätigkeit $(F(4,138)=0,76 ; p=, 552$; $\left.\eta_{p}^{2}=, 022\right)$.

Analog zu den vorhergehenden Befunden war auch die Time to Collision bei Bremsreaktion signifikant geringer, wenn die Teilnehmer nicht durch den Ball vorgewarnt worden waren $\left(F(1,138)=18,62 ; p<, 001 ; \eta_{p}^{2}=, 119\right.$, siehe Tabelle 24, S. 75). Obwohl die Teilnehmer beim Schreiben der Nachricht tendenziell bei geringerer Time to Collision bremsten, konnte zwischen den Nebentätigkeiten, und auch im Vergleich zur Baseline, kein statistisch relevanter Unterschied gefunden $\left(F(4,138)=1,76 ; p=, 140 ; \eta_{p}^{2}=, 049\right)$. Auch Interaktion zwischen Vorinformation und Nebentätigkeit konnte nicht gefunden werden $\left(F(4,138)=0,74 ; p=, 568 ; \eta_{p}^{2}=, 021\right)$.

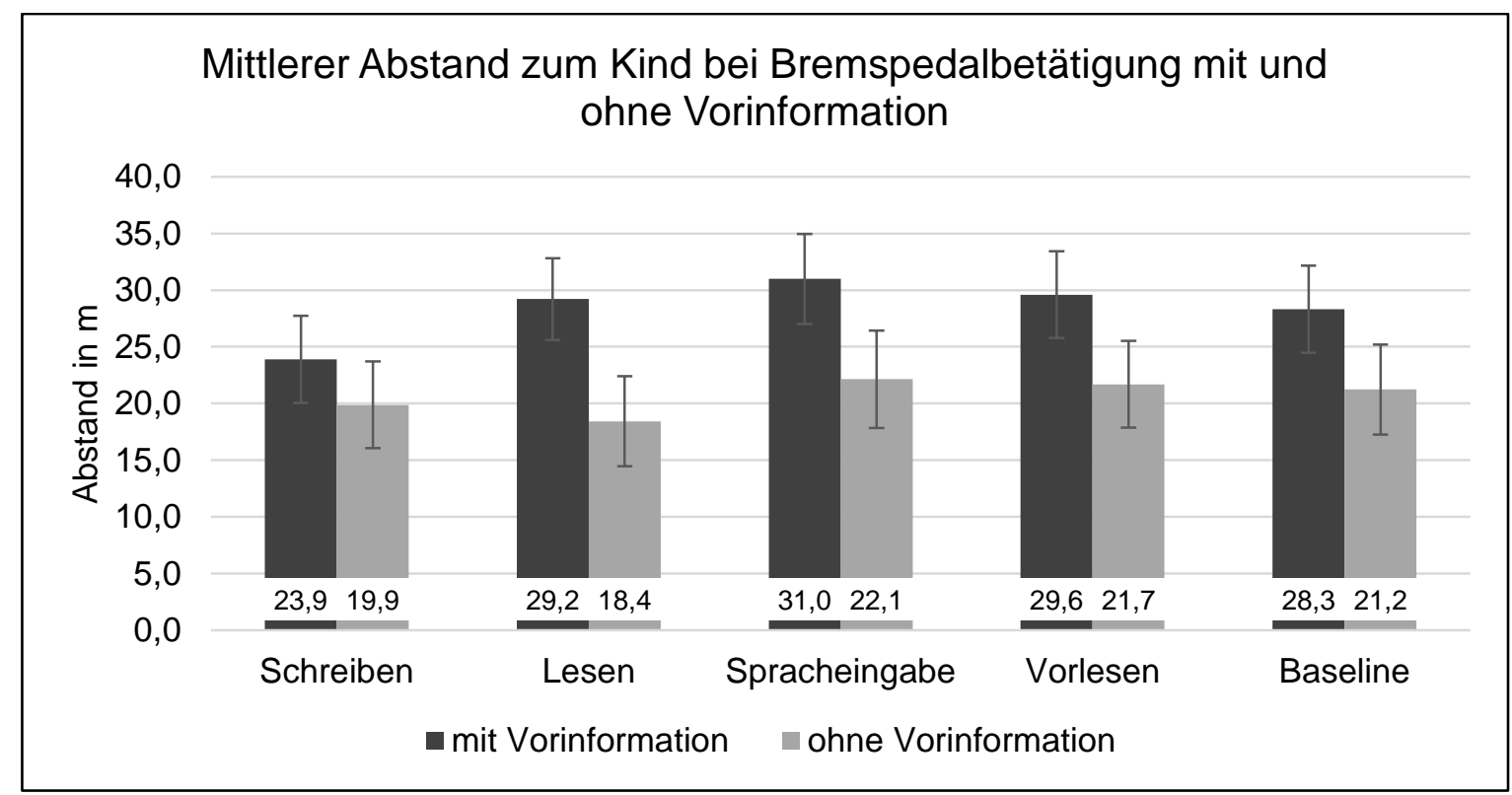

Abbildung 35: Abstand zum Kind bei Bremspedalbetätigung mit und ohne Vorinformation, Fahrdaten ( $N=148$ Situationen, Fehlerbalken 95\% Konfidenzintervall). 
Tabelle 24: Reaktionszeit nach Sichtbarkeit des Balles, sowie Abstand und Time to Collision zum Kind bei einer Reaktion mit und ohne Vorinformation und die verschiedenen Nebenaufgabenbedingungen, Fahrdaten.

\begin{tabular}{|c|c|c|c|c|c|c|c|c|c|}
\hline & & \multicolumn{4}{|c|}{ Mit Vorinformation } & \multicolumn{4}{|c|}{ Ohne Vorinformation } \\
\hline & & $N$ & $M$ & $S D$ & $M D$ & $N$ & $M$ & $S D$ & $M D$ \\
\hline \multicolumn{10}{|c|}{ Reaktionszeit } \\
\hline \multirow{5}{*}{$\begin{array}{c}\text { Reduktion } \\
\text { des } \\
\text { Gaspedal- } \\
\text { drucks } \\
N=146\end{array}$} & Schreiben & 15 & 2,31 & 0,75 & 2,32 & 15 & 2,95 & 0,81 & 3,02 \\
\hline & Lesen & 17 & 2,12 & 0,96 & 2,02 & 14 & 3,12 & 0,55 & 3,13 \\
\hline & Spracheingabe & 13 & 2,17 & 0,54 & 2,03 & 12 & 2,32 & 1,19 & 2,99 \\
\hline & Vorlesen & 14 & 1,82 & 0,58 & 1,92 & 14 & 2,40 & 0,98 & 2,93 \\
\hline & Baseline & 17 & 1,70 & 0,41 & 1,68 & 15 & 2,62 & 0,89 & 3,00 \\
\hline \multirow{5}{*}{$\begin{array}{c}\text { Brems- } \\
\text { pedal- } \\
\text { betätigung } \\
N=148\end{array}$} & Schreiben & 15 & 3,06 & 0,56 & 3,02 & 17 & 3,44 & 0,69 & 3,45 \\
\hline & Lesen & 17 & 2,81 & 0,56 & 3,00 & 14 & 3,60 & 0,36 & 3,49 \\
\hline & Spracheingabe & 14 & 2,68 & 0,38 & 2,69 & 12 & 3,41 & 0,24 & 3,43 \\
\hline & Vorlesen & 15 & 2,83 & 0,42 & 2,83 & 15 & 3,33 & 0,20 & 3,27 \\
\hline & Baseline & 15 & 2,66 & 0,58 & 2,55 & 14 & 3,29 & 0,16 & 3,33 \\
\hline \multicolumn{10}{|c|}{ Abstand } \\
\hline \multirow{5}{*}{$\begin{array}{c}\text { Reduktion } \\
\text { des } \\
\text { Gaspedal- } \\
\text { drucks } \\
N=146\end{array}$} & Schreiben & 15 & 35,51 & 10,58 & 37,76 & 15 & 27,54 & 11,44 & 26,82 \\
\hline & Lesen & 17 & 38,88 & 13,86 & 42,46 & 14 & 25,11 & 9,36 & 24,33 \\
\hline & Spracheingabe & 13 & 38,74 & 6,93 & 41,57 & 12 & 37,97 & 16,71 & 33,73 \\
\hline & Vorlesen & 14 & 44,05 & 8,47 & 43,76 & 14 & 35,91 & 13,78 & 30,36 \\
\hline & Baseline & 17 & 42,82 & 5,02 & 44,09 & 15 & 29,65 & 15,83 & 25,19 \\
\hline \multirow{5}{*}{$\begin{array}{c}\text { Brems- } \\
\text { pedal- } \\
\text { betätigung } \\
N=148\end{array}$} & Schreiben & 15 & 23,88 & 9,24 & 26,40 & 17 & 19,88 & 10,52 & 21,29 \\
\hline & Lesen & 17 & 29,21 & 8,36 & 33,34 & 14 & 18,43 & 6,77 & 19,52 \\
\hline & Spracheingabe & 14 & 30,99 & 6,71 & 31,38 & 12 & 22,14 & 6,48 & 21,87 \\
\hline & Vorlesen & 15 & 29,60 & 6,74 & 31,35 & 15 & 21,69 & 7,22 & 23,40 \\
\hline & Baseline & 15 & 28,32 & 8,83 & 31,42 & 14 & 21,23 & 6,69 & 20,07 \\
\hline \multicolumn{10}{|c|}{ Time to Collision } \\
\hline \multirow{5}{*}{$\begin{array}{c}\text { Reduktion } \\
\text { des } \\
\text { Gaspedal- } \\
\text { drucks } \\
N=146\end{array}$} & Schreiben & 15 & 2,35 & 67 & 2,41 & 15 & 1,88 & ,66 & 1,86 \\
\hline & Lesen & 17 & 2,67 & 99 & 2,72 & 14 & 1,79 & ,76 & 1,79 \\
\hline & Spracheingabe & 13 & 2,67 & ,60 & 2,69 & 12 & 2,72 & 1,15 & 2,65 \\
\hline & Vorlesen & 14 & 3,15 & ,77 & 3,01 & 14 & 2,47 & ,94 & 2,33 \\
\hline & Baseline & 17 & 2,66 & ,36 & 2,78 & 15 & 2,05 & 1,23 & 1,72 \\
\hline \multirow{5}{*}{$\begin{array}{c}\text { Brems- } \\
\text { pedal- } \\
\text { betätigung } \\
N=148\end{array}$} & Schreiben & 15 & 1,63 & ,67 & 1,67 & 17 & 1,40 & 74 & 1,50 \\
\hline & Lesen & 17 & 2,08 & ,72 & 1,84 & 14 & 1,33 &, 57 & 1,42 \\
\hline & Spracheingabe & 14 & 2,19 & ,62 & 2,13 & 12 & 1,67 & ,73 & 1,49 \\
\hline & Vorlesen & 15 & 2,14 & ,60 & 2,26 & 15 & 1,58 & ,72 & 1,65 \\
\hline & Baseline & 15 & 1,83 & ,57 & 1,89 & 14 & 1,51 & ,67 & 1,38 \\
\hline
\end{tabular}




\subsubsection{Besondere Verkehrssituationen - Baustelle}

\section{Unfälle und Beinaheunfälle}

Anders als beim zuvor beschriebenen Szenario kamen an der Baustelle alle Fahrer rechtzeitig zum Stehen, egal ob mit oder ohne Hinweisschild. Es wurden keine Unfälle oder Beinaheunfälle beobachtet.

\section{Reduktion Gaspedaldruck}

Auch bei der Baustelle wurde analysiert, wann die Teilnehmer den Gaspedaldruck reduzierten. Mit dem Schild als Vorinformation wurde signifikant früher das Gas reduziert als ohne Schild als Vorinformation $(U=1.668,5 ; p<, 001$, siehe Tabelle 25). Allerdings fand sich kein signifikanter Unterschied zwischen den Nebentätigkeiten bezogen auf die Reduktion des Gaspedaldrucks $(H(4)=0,850 ; p=, 932)$.

Analog dazu war der Abstand zur Baustelle bei Reduktion des Gaspedaldrucks signifikant größer, wenn zuvor das Baustellenschild auf die Baustelle hingewiesen hatte $(U=1.766,0 ; p=, 001$, siehe Tabelle 25, S. 78). Besonders deutlich ist dieser Effekt in den Bedingungen Lesen und Vorlesen ausgeprägt. Unabhängig davon welche Nebentätigkeit ausgeführt wurde oder auch in der Bedingung ohne Nebentätigkeit wurde der Gaspedaldruck in einem ähnlichen Abstand zur Baustelle vermindert $(H(4)=1,29$; $p=$,864). Allerdings ist auffällig, dass, wenn keine Vorinformation erfolgte, die Teilnehmer bei Schreiben einer Nachricht in deutlich größerem Abstand zur Baustelle vom Gas gingen als die Teilnehmer in den anderen Bedingungen.

Auch die Time to Collision bei Reduktion des Gaspedaldrucks war signifikant größer, wenn die Baustelle zuvor durch das Schild angekündigt wurde $(U=1.822,0 ; p=, 002$, siehe Tabelle 25, S. 78). Zwischen den Nebentätigkeiten bzw. der Baseline besteht kein signifikanter Unterschied in Bezug auf die Time to Collision bei der der Gaspedaldruck reduziert wurde $(H(4)=0,65 ; p=, 958)$.

\section{Bremspedalbetätigung}

Anders als bei Reduktion des Gaspedaldrucks schien das Baustellenschild keinen erkennbaren Effekt auf die Bremsreaktion der Teilnehmer zu haben (siehe Tabelle 25, S. 78). So zeigte die Varianzanalyse für den Zeitpunkt der Bremsreaktion, dass mit dem Schild als Vorinformation ebenso spät gebremst wurde als ohne Schild $(F(1,141)=2,26$; $\left.p=, 135 ; \eta_{p}^{2}=, 016\right)$. Eine Erklärung mag in der eher geringen Kritikalität der Situation liegen, die dazu führte, dass die Teilnehmer tatsächlich erst bei Sichtbarkeit der Baustelle bremsten. Für die einzelnen Nebentätigkeiten ergab sich allerdings ein signifikanter Effekt auf den Zeitpunkt der Bremsung $(F(4,141)=2,86 ; p=, 026$; $\left.\eta_{p}^{2}=, 075\right)$. Die post-hoc Vergleiche ergaben jedoch keine signifikanten Unterschiede zwischen den einzelnen Nebentätigkeiten (kleinstes $p=, 052$ ). Auch konnte keine Interaktion zwischen Vorinformation und Nebentätigkeit gefunden werden $\left(F(4,141)=2,30 ; p=, 062 ; \eta_{p}^{2}=, 061\right)$.

Ähnliche Befunde zeigten sich bei der Betrachtung des Abstands zur Baustelle bei Bremsreaktion. Das Hinweisschild auf die Baustelle hatte keinen Effekt auf diesen Abstand $(U=2.505,0 ; p=, 207$, siehe Tabelle 25, S. 78). Allerdings fanden sich signifikante Unterschiede zwischen den Nebentätigkeiten $(H(4)=12,03 ; p=, 017)$, so bremsten die Teilnehmer beim manuellen Lesen am Mobiltelefon mit geringerem Abstand als diejenigen die keine Nebenaufgabe hatten $(p=, 017)$. 
Auch auf die Time to Collision bei der Bremsreaktion hatte die Präsenz des Baustellenschildes keinen Einfluss $\left(F(1,141)=2,55 ; p=, 112 ; \eta^{2}{ }_{p}=, 018\right.$, siehe Tabelle 25, S. 78). Ebenso wurde kein Unterschied zwischen den einzelnen Nebentätigkeiten gefunden $\left(F(4,141)=1,93 ; p=, 109 ; \eta_{p}^{2}=, 049\right)$. Allerdings bestand eine signifikante Interaktion zwischen der Art der Nebentätigkeit und der Vorinformation $(F(4,141)=2,51$; $\left.p=, 045 ; \eta^{2 p}=, 066\right)$. Bei den Teilnehmern, die Nachrichten schrieben, war die Time to Collision bei Bremsung kleiner mit Vorinformation im Vergleich zu keiner Vorinformation. Bei der Baselinegruppe, d.h. den Teilnehmer, die keine Nebenaufgaben bearbeitet haben, war es genau umgekehrt. Die Time to Collision Bremsung war mit Vorinformation größer als ohne. 
Tabelle 25: Reaktionszeit nach Sichtbarkeit des Baustellenschildes, sowie Abstand und Time to Collision zur Baustelle bei einer Reaktion mit und ohne Vorinformation und die verschiedenen Nebenaufgabenbedingungen, Fahrdaten.

\begin{tabular}{|c|c|c|c|c|c|c|c|c|c|}
\hline & & \multicolumn{4}{|c|}{ Mit Vorinformation } & \multicolumn{4}{|c|}{ Ohne Vorinformation } \\
\hline & & $N$ & $M$ & $S D$ & $M D$ & $N$ & $M$ & $S D$ & $M D$ \\
\hline \multicolumn{10}{|c|}{ Reaktionszeit } \\
\hline \multirow{5}{*}{$\begin{array}{c}\text { Reduktion } \\
\text { des } \\
\text { Gaspedal- } \\
\text { drucks } \\
N=146\end{array}$} & Schreiben & 13 & 7,41 & 4,74 & 9,15 & 14 & 7,63 & 3,79 & 7,46 \\
\hline & Lesen & 11 & 6,26 & 4,50 & 5,42 & 17 & 9,09 & 4,20 & 10,82 \\
\hline & Spracheingabe & 15 & 8,76 & 3,02 & 9,50 & 15 & 8,56 & 3,97 & 10,12 \\
\hline & Vorlesen & 13 & 4,96 & 3,40 & 4,87 & 15 & 10,59 & 3,52 & 11,82 \\
\hline & Baseline & 16 & 6,69 & 2,93 & 7,08 & 16 & 9,98 & 2,58 & 10,93 \\
\hline \multirow{5}{*}{$\begin{array}{c}\text { Brems- } \\
\text { pedal- } \\
\text { betätigung } \\
N=148\end{array}$} & Schreiben & 15 & 11,59 & 1,62 & 11,18 & 14 & 11,29 & 2,17 & 12,24 \\
\hline & Lesen & 12 & 11,85 & 1,80 & 11,87 & 17 & 12,55 & 1,59 & 12,78 \\
\hline & Spracheingabe & 15 & 12,86 & 1,13 & 12,93 & 15 & 12,21 & 1,42 & 12,22 \\
\hline & Vorlesen & 13 & 11,19 & 1,88 & 11,02 & 16 & 12,91 & 1,33 & 12,73 \\
\hline & Baseline & 17 & 11,08 & 1,71 & 11,43 & 17 & 11,64 & 1,74 & 12,23 \\
\hline \multicolumn{10}{|c|}{ Abstand } \\
\hline \multirow{5}{*}{$\begin{array}{c}\text { Reduktion } \\
\text { des } \\
\text { Gaspedal- } \\
\text { drucks } \\
N=146\end{array}$} & Schreiben & 13 & 152,2 & 72,10 & 126,0 & 14 & 150,6 & 57,26 & 147,4 \\
\hline & Lesen & 11 & 172,9 & 68,01 & 183,7 & 17 & 132,7 & 59,43 & 94,54 \\
\hline & Spracheingabe & 15 & 138,1 & 49,95 & 138 & 15 & 135,3 & 61,73 & 99,41 \\
\hline & Vorlesen & 13 & 190,4 & 56,29 & 189,3 & 15 & 115,8 & 45,89 & 95,27 \\
\hline & Baseline & 16 & 165,2 & 44,74 & 161,8 & 16 & 114,1 & 35,74 & 97,11 \\
\hline \multirow{5}{*}{$\begin{array}{c}\text { Brems- } \\
\text { pedal- } \\
\text { betätigung } \\
N=148\end{array}$} & Schreiben & 15 & 83,14 & 15,56 & 77,21 & 14 & 94,13 & 27,37 & 79,31 \\
\hline & Lesen & 12 & 83,78 & 18,09 & 77,03 & 17 & 79,78 & 16,82 & 75,56 \\
\hline & Spracheingabe & 15 & 80,82 & 15,54 & 81,38 & 15 & 80,74 & 11,12 & 80,70 \\
\hline & Vorlesen & 13 & 96,41 & 28,36 & 88,78 & 16 & 81,33 & 9,46 & 83,30 \\
\hline & Baseline & 17 & 99,55 & 18,49 & 94,51 & 17 & 86,89 & 19,45 & 81,44 \\
\hline \multicolumn{10}{|c|}{ Time to Collision } \\
\hline \multirow{5}{*}{$\begin{array}{c}\text { Reduktion } \\
\text { des } \\
\text { Gaspedal- } \\
\text { drucks } \\
N=146\end{array}$} & Schreiben & 13 & 9,40 & 4,31 & 7,30 & 14 & 9,37 & 3,27 & 8,94 \\
\hline & Lesen & 11 & 10,47 & 3,96 & 11,44 & 17 & 8,53 & 3,63 & 6,47 \\
\hline & Spracheingabe & 15 & 9,37 & 3,89 & 10,02 & 15 & 8,76 & 4,29 & 6,86 \\
\hline & Vorlesen & 13 & 11,89 & 3,80 & 11,23 & 15 & 7,67 & 2,63 & 6,86 \\
\hline & Baseline & 16 & 10,50 & 2,98 & 10,42 & 16 & 7,18 & 2,29 & 6,50 \\
\hline \multirow{5}{*}{$\begin{array}{c}\text { Brems- } \\
\text { pedal- } \\
\text { betätigung } \\
N=148\end{array}$} & Schreiben & 15 & 5,32 & 1,10 & 4,83 & 14 & 6,16 & 1,39 & 5,64 \\
\hline & Lesen & 12 & 5,53 & 1,48 & 5,23 & 17 & 5,44 & 1,33 & 5,35 \\
\hline & Spracheingabe & 15 & 5,73 & 1,42 & 5,79 & 15 & 5,26 & ,81 & 5,35 \\
\hline & Vorlesen & 13 & 6,59 & 2,18 & 5,84 & 16 & 5,71 & ,78 & 5,61 \\
\hline & Baseline & 17 & 6,84 & 1,68 & 6,66 & 17 & 5,60 & 1,42 & 5,19 \\
\hline
\end{tabular}




\subsubsection{Besondere Verkehrssituationen - Schild $30 \mathrm{~km} / \mathrm{h}$ Geschwindigkeitsbegrenzung}

\section{Reduktion Gaspedaldruck und Bremspedalbetätigung}

Es wurden an zwei Stellen in der Simulation Geschwindigkeitsbegrenzungen von $30 \mathrm{~km} / \mathrm{h}$ implementiert, einmal in einer parkähnlichen Umgebung bei der das Schild durch Verdeckung relativ spät zu sehen war und im Wohn- und Geschäftsviertel auf einer langen Geraden mit guter Einsehbarkeit des Schildes. Um zu prüfen, wie schnell die Teilnehmer auf die neue Geschwindigkeitsbegrenzung reagieren, wurde auch hier die Zeit ab Sichtbarkeit des Schildes bis zur Reduktion des Gaspedaldrucks bzw. bis zur Bremspedalbetätigung analysiert.

In Bezug auf die Reduktion des Gaspedaldrucks wurden für das Park-Szenario signifikante Unterschiede zwischen den Nebentätigkeiten gefunden $(H(4)=19,64$; $p=, 001)$. In der Baseline wurde signifikant schneller mit einer Reduktion des Gaspedaldrucks auf das Schild reagiert als beim Schreiben einer Textnachricht $(p=, 017)$ oder bei Nutzung der Vorlesefunktion $(p<, 001)$, (siehe Tabelle 26, S. 80). Auch für die Spracheingabe zeigt sich im Vergleich zur Baseline die gleiche Tendenz, auch wenn der Unterschied statistisch nicht signifikant war. Bei der langen Geraden im Wohn- und Geschäftsviertel zeigte sich kein Unterschied zwischen den Nebentätigkeiten und der Baseline $(H(4)=8,05 ; p=, 090)$. Der Grund ist möglicherweise die große Varianz in den Reaktionszeiten der Teilnehmer innerhalb ihrer Gruppen. Aufgrund der guten Sichtbarkeit des Schildes stand ein relativ großes Zeitfenster für eine Reaktion zur Verfügung stand. Wahrscheinlich überdecken individuelle Fahrstrategien (generell frühes / spätes vom-Gas-Gehen) hier etwaige Effekte der Nebenaufgaben.

Vergleichbare Ergebnisse zeigten sich auch für die Betätigung des Bremspedals. Im Park wurde über alle Nebentätigkeiten hinweg ein signifikanter Unterschied in der Zeit bis zur Bremspedalbetätigung gefunden $(H(4)=15,18 ; p=, 004$, siehe Tabelle 26, S. 80). Die deskriptiven Ergebnisse zeigen, dass besonders die Teilnehmer die eine Textnachricht verfassten oder einsprachen, später bremsten im Vergleich zu den Teilnehmern der Baselinegruppe. Das würde für eine geringere Ablenkung der Baselinegruppe sprechen. Allerdings waren die Unterschiede nicht signifikant (kleinstes $p=, 051$ Baseline vs. Schreiben). Im Wohn- und Geschäftsviertel wurde im Schnitt bei allen Nebentätigkeiten sowie der Baseline zu vergleichbaren Zeitpunkten gebremst $(H(4)=4,65 ; p=, 325)$.

Dauer bis eine Geschwindigkeitsreduktion von minus 20\% erreicht wurde (Einstellen der Zielgeschwindigkeit)

Als weiterer Indikator für die Reaktion auf die veränderte Geschwindigkeitsbegrenzung wurde untersucht, wie lange es dauert bis die Teilnehmer ihre Geschwindigkeit um mindestens $20 \%$ (z.B. von $50 \mathrm{~km} / \mathrm{h}$ auf $40 \mathrm{~km} / \mathrm{h}$ ) reduziert hatten, ausgehend vom Zeitpunkt der Reduktion des Gaspedaldrucks. Sowohl im Park-Szenario als auch im Wohn- und Geschäftsviertel wurde kein signifikanter Unterschied zwischen den einzelnen Nebentätigkeiten und der Baseline gefunden (Park: $H(4)=1,96 ; p=, 744$; Wohn-/Geschäftsviertel: $H(4)=7,14 ; p=, 129$, siehe Tabelle 26, S. 80). Gleiche Ergebnisse ergaben sich mit der Bremsreaktion als Referenzpunkt. Auch hier wurde weder für den Park noch das Wohn- und Geschäftsviertel ein Unterschied zwischen den Nebenaufgabenbedingungen in Bezug auf die Zeit bis zur Reduktion der Geschwindigkeit um 20\% gefunden (Park: $H(4)=3,08 ; p=, 545$; Wohn-/Geschäftsviertel: $H(4)=8,42 ; p=, 078)$ 
Tabelle 26: Reaktionszeit nach Sichtbarkeit des $30 \mathrm{~km} / \mathrm{h}$ Schildes, sowie Dauer bis zu einer Geschwindigkeitsreduktion um 20\% im Park und im Wohn-und Geschäftsviertel und die verschiedenen Nebenaufgabenbedingungen, Fahrdaten.

\begin{tabular}{|c|c|c|c|c|c|c|c|c|c|}
\hline & & \multicolumn{4}{|c|}{ Park } & \multicolumn{4}{|c|}{ Wohn-/Geschäftsviertel } \\
\hline & & $N$ & $M$ & $S D$ & $M D$ & $N$ & $M$ & $S D$ & $M D$ \\
\hline \multicolumn{10}{|c|}{ Reaktionszeit } \\
\hline \multirow{5}{*}{$\begin{array}{c}\text { Reduktion } \\
\text { des } \\
\text { Gaspedal- } \\
\text { drucks } \\
N=146\end{array}$} & Schreiben & 24 & 2,52 & 2,10 & 1,98 & 30 & 6,07 & 3,70 & 5,44 \\
\hline & Lesen & 26 & 2,10 & 2,62 & 1,57 & 31 & 5,68 & 5,06 & 3,25 \\
\hline & Spracheingabe & 23 & 2,74 & 3,19 & 1,80 & 29 & 7,90 & 4,76 & 8,27 \\
\hline & Vorlesen & 26 & 3,25 & 2,18 & 3,49 & 28 & 5,39 & 3,38 & 4,18 \\
\hline & Baseline & 30 & 1,27 & 1,05 & 1,14 & 32 & 6,94 & 2,46 & 7,93 \\
\hline \multirow{5}{*}{$\begin{array}{c}\text { Brems- } \\
\text { pedal- } \\
\text { betätigung } \\
N=148\end{array}$} & Schreiben & 17 & 4,02 & 2,09 & 3,37 & 27 & 10,60 & 2,05 & 10,13 \\
\hline & Lesen & 23 & 2,83 & 1,12 & 2,48 & 24 & 9,68 & 3,72 & 9,57 \\
\hline & Spracheingabe & 24 & 3,91 & 1,95 & 3,52 & 26 & 10,94 & 2,19 & 10,32 \\
\hline & Vorlesen & 23 & 3,71 & 1,52 & 4,23 & 26 & 10,44 & 2,89 & 9,93 \\
\hline & Baseline & 32 & 2,57 & ,83 & 2,59 & 30 & 9,94 & 2,15 & 9,94 \\
\hline \multicolumn{10}{|c|}{ Dauer } \\
\hline \multirow{5}{*}{$\begin{array}{c}\text { Reduktion } \\
\text { des } \\
\text { Gaspedal- } \\
\text { drucks } \\
N=146\end{array}$} & Schreiben & 18 & 3,40 & 2,65 & 7,10 & 27 & 6,88 & 4,40 & 2,05 \\
\hline & Lesen & 21 & 3,67 & 3,24 & 6,68 & 30 & 6,87 & 4,08 & 2,55 \\
\hline & Spracheingabe & 22 & 3,17 & 1,74 & 2,87 & 25 & 4,88 & 4,08 & 2,67 \\
\hline & Vorlesen & 18 & 3,30 & 1,48 & 7,08 & 27 & 7,38 & 4,19 & 3,11 \\
\hline & Baseline & 29 & 2,89 & 1,66 & 5,58 & 32 & 6,33 & 3,43 & 2,52 \\
\hline \multirow{5}{*}{$\begin{array}{c}\text { Brems- } \\
\text { pedal- } \\
\text { betätigung } \\
N=148\end{array}$} & Schreiben & 17 & 1,31 & 1,29 & ,92 & 27 & 1,66 & 3,09 & ,85 \\
\hline & Lesen & 22 & 1,40 & 1,43 & 1,00 & 24 & 1,48 & 1,66 & 1,17 \\
\hline & Spracheingabe & 24 & 1,38 & 1,35 &, 93 & 26 & 1,07 & ,68 & ,89 \\
\hline & Vorlesen & 22 & 1,51 & 1,23 & 1,13 & 24 & 1,57 & 1,80 & 1,00 \\
\hline & Baseline & 32 & 1,24 &, 58 & 1,05 & 30 & 2,64 & 2,74 & 1,34 \\
\hline
\end{tabular}

\subsubsection{Beanspruchung und der Einfluss von Kontextvariablen auf das Verfassen von Textnachrichten}

Die generelle von der Fahrstrecke ausgehende Beanspruchung wurde anhand von sechs ausgewählten Situationen mittels Rating Scale for Mental Effort überprüft (siehe Tabelle 27). Hierbei sollten die Teilnehmer auf einer Skala von 0 - absolut keine Anstrengung bis 150 - mehr als extreme Anstrengung ihre Beanspruchung in der jeweiligen Situation einschätzen. Die meisten Situationen wurden als wenig bis etwas anstrengend eingeschätzt. Auch in den Situationen, in denen sich im weiteren Verlauf kritische Situationen ergeben hatten, wurden als wenig bzw. maximal etwas anstrengend eingeschätzt (Situation Nr. 3 \& Nr. 5). Entsprechend signalisierten die Teilnehmer auf Nachfrage eine generell hohe Bereitschaft, in den sechs ausgewählten Situationen Textnachrichten zu verfassen. In den meisten Situationen würde mindestens die Hälfte, bis teilweise mehr als $90 \%$ der Teilnehmer eine Textnachricht schreiben. Lediglich in der etwas stärker beanspruchenden Situation Nr. 6 waren nur 13,4\% der Teilnehmer dazu bereit. Die statistische Überprüfung des Zusammenhangs zwischen der 
selbsteingeschätzten Beanspruchung in den sechs Situationen und der Bereitschaft, in den Situationen eine Textnachricht zu schreiben zeigt, dass eine hohe subjektive Beanspruchung mit einer reduzierten Bereitschaft zum Verfassen einer Textnachricht einherging (Spearman Rho Korrelation: $r=-, 714 ; p<, 111$, Abbildung 36, links). Die Erhöhung des Unfallrisikos durch das Schreiben von Textnachrichten wurde für die meisten Situationen als relativ gering eingestuft (max. 50,2\% für Situation Nr. 2). Lediglich für die Situation Nr. 6, die als etwas stärker anstrengend eingeschätzt wurde und bei der nur wenige Teilnehmer eine Textnachricht schreiben würden, wurde eine mittlere Risikoerhöhung von beinahe $70 \%$ angegeben. Auch hier zeigt die statistische Prüfung einen Zusammenhang zwischen der wahrgenommenen Erhöhung des Unfallrisikos und der Bereitschaft zu Texten (siehe Abbildung 36, rechts). Eine subjektiv stärkere Risikoerhöhung geht mit einer geringeren Bereitschaft zum Schreiben einer Textnachricht einher $(r=-, 943 ; p<, 001)$.

Tabelle 27: Einschätzung bei der Beanspruchungsmessung (RSME, Skala 0 - 150), Anteil der Teilnehmer die Texten würden in \% und die Einschätzung der Erhöhung des Risikos eines Unfalls in \% für die sechs Situationen aus der Simulation, Interviewdaten $(N=82)$.

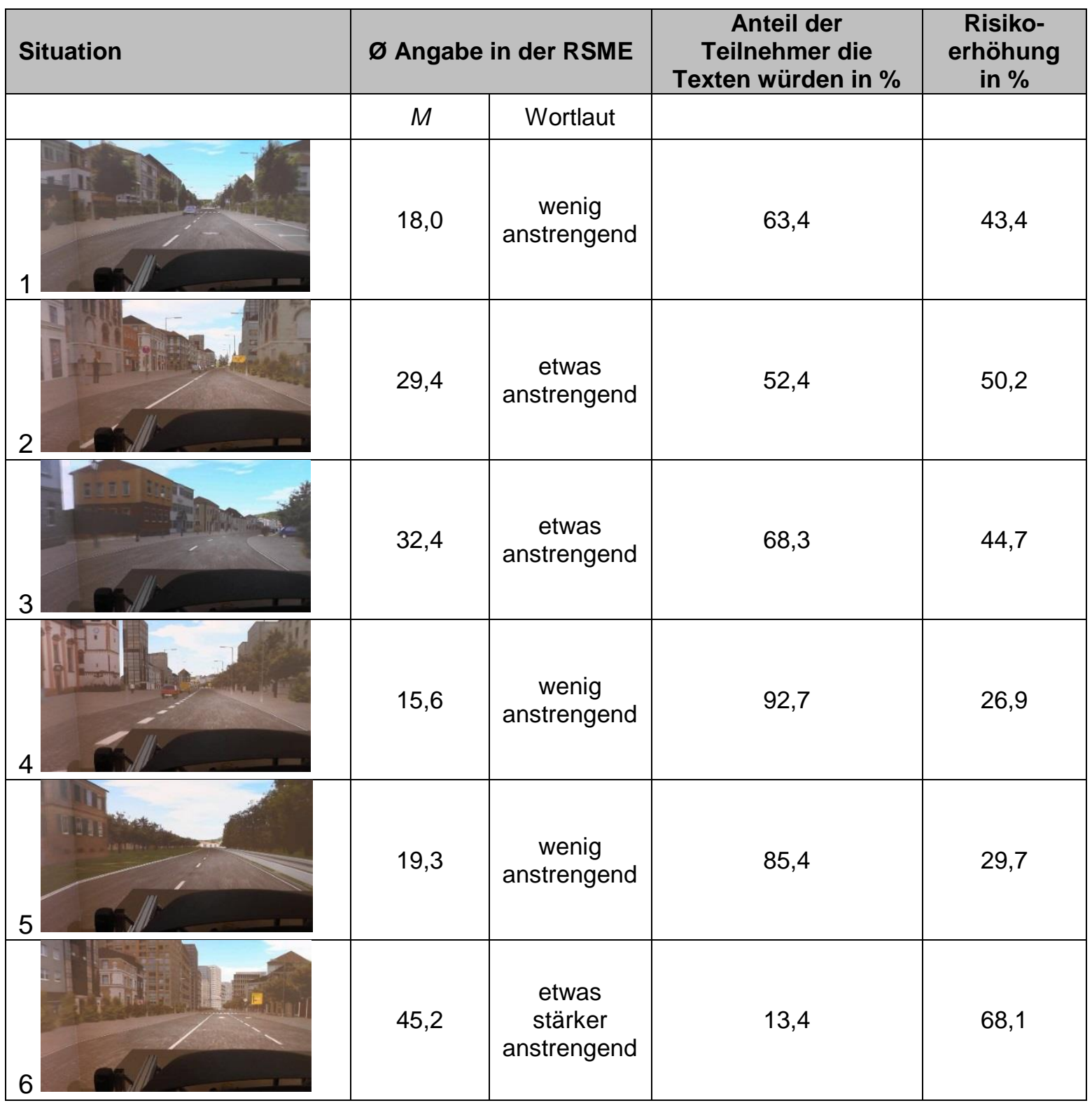




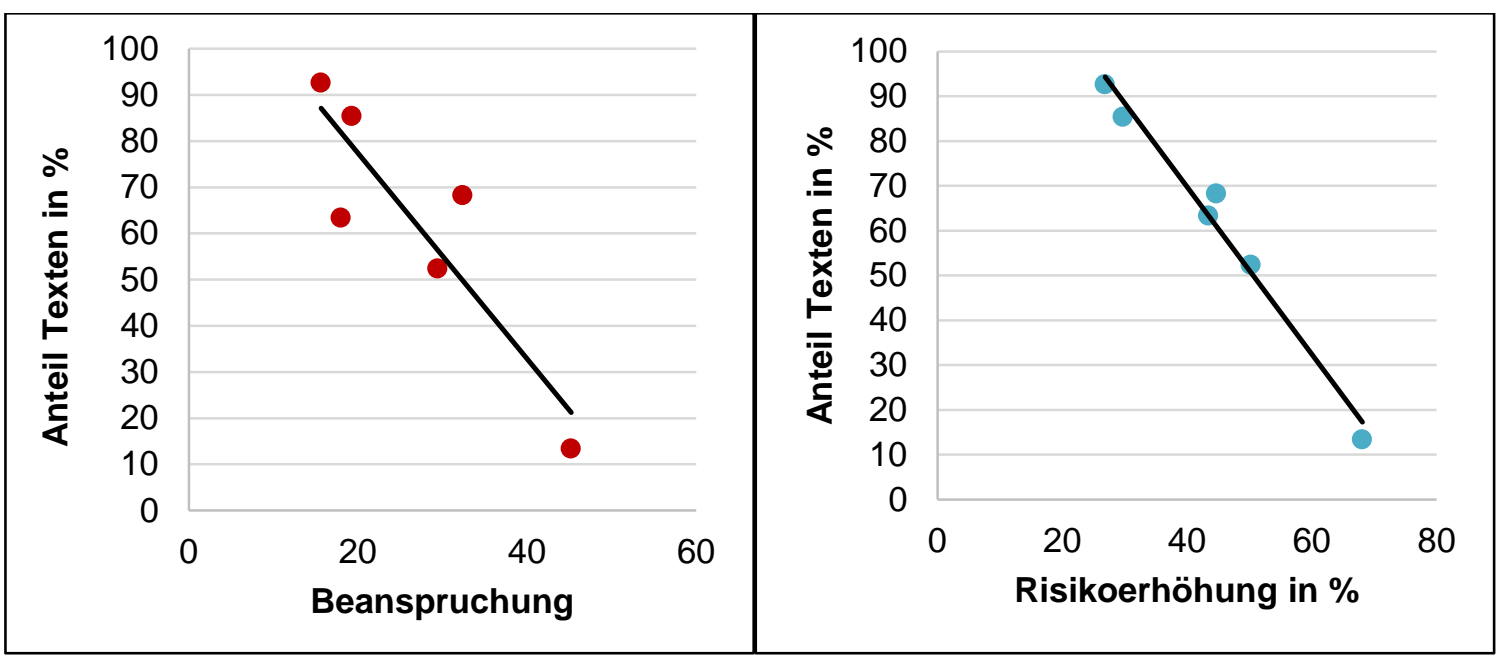

Abbildung 36: links: Spearman Rho Korrelation zwischen Beanspruchung und Häufigkeit Texting $(N=6)$, rechts: Spearman Rho Korrelation zwischen Häufigkeit Texting und Risikoerhöhung, Interviewdaten $(N=6)$.

Außerdem wurden die Teilnehmer zu den situativen Merkmalen befragt, die jeweils dazu führen würden, dass sie zum Verfassen einer Textnachricht in der gezeigten Situation bereit wären. Am häufigsten wurde genannt, dass keine oder nur wenige andere Verkehrsteilnehmer zugegen waren (siehe Abbildung 37). Beispielhaft ist eine Antwort wie etwa „freier Fußweg, keine Passanten“. Außerdem wurde, ähnlich wie in der Interviewstudie, auch wiederholt angemerkt, dass es sich um eine breite und gerade Strecke handeln würde, oder es sich insgesamt um eine übersichtliche Situation handele, mit Aussagen wie z.B. "gut einsehbar".

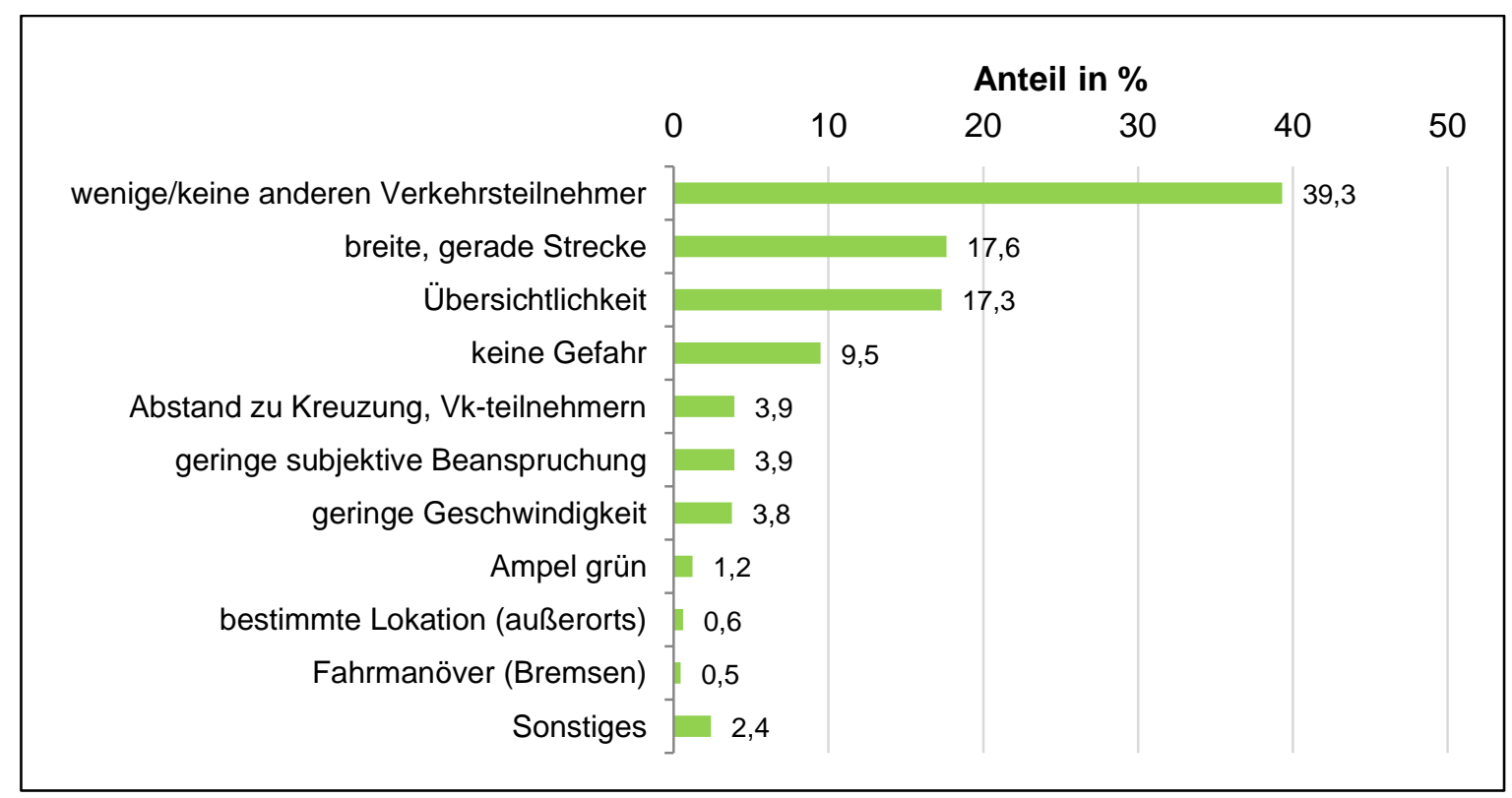

Abbildung 37: Nennungen (in \%) für Argumente warum Teilnehmer eine Textnachricht verfassen würden, Interviewdaten $(N=82)$.

Als Gründe dafür, sich in einer der gezeigten Situation gegen das Verfassen einer Textnachricht zu entscheiden wurde mit sehr großer Mehrheit genannt, dass es um eine enge, kurvige oder komplexe Straße handele (siehe Abbildung 38), mit Aussagen wie z.B. „schmale Straße“ oder auch „Kreuzungsbereich“. Als weitere Gründe wurden die Präsenz anderer Verkehrsteilnehmer, z.B. „Gefahr durch plötzlich auftauchende 
Menschen“, sowie allgemein die Tatsache, dass die Situation Konzentration erfordere, genannt.

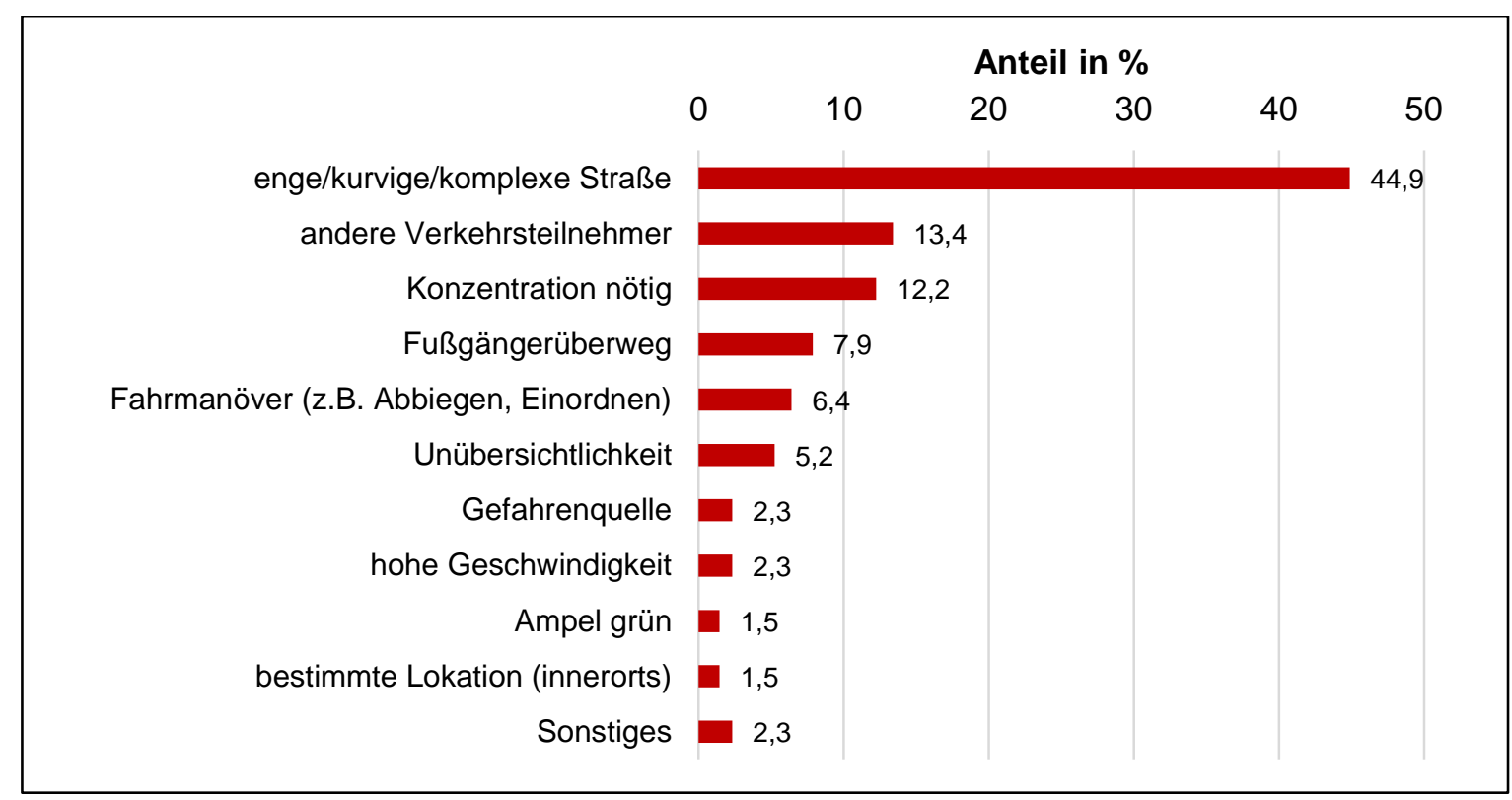

Abbildung 38: Nennungen (in \%) für Argumente warum Teilnehmer keine Textnachricht verfassen würden, Interviewdaten $(N=82)$.

Wie in der Interviewstudie wurde den Teilnehmer in der Folge wieder die komplementäre Frage gestellt, welches Merkmal in der jeweiligen Situation anders sein müsste, damit sie das Verfassen einer Textnachricht unterlassen (wenn zuvor eine Bereitschaft zum Texten signalisiert wurde) oder mit dem Schreiben einer Textnachricht beginnen (wenn zuvor keine Bereitschaft bestand). Auf die Frage „Was müsste anders sein, damit sie keine Textnachricht in der Situation verfassen?" wurde mit knapp 60\% die Präsenz von anderen Verkehrsteilnehmern genannt (siehe Abbildung 39) z.B. „Kinder am Straßenrand“ oder "mehr Autos links und rechts“. Mit großem Abstand wurden dann noch die Enge bzw. Kurvigkeit der Straße, sowie andere Gefahrenquellen, z.B. "Baustelle“, genannt. 


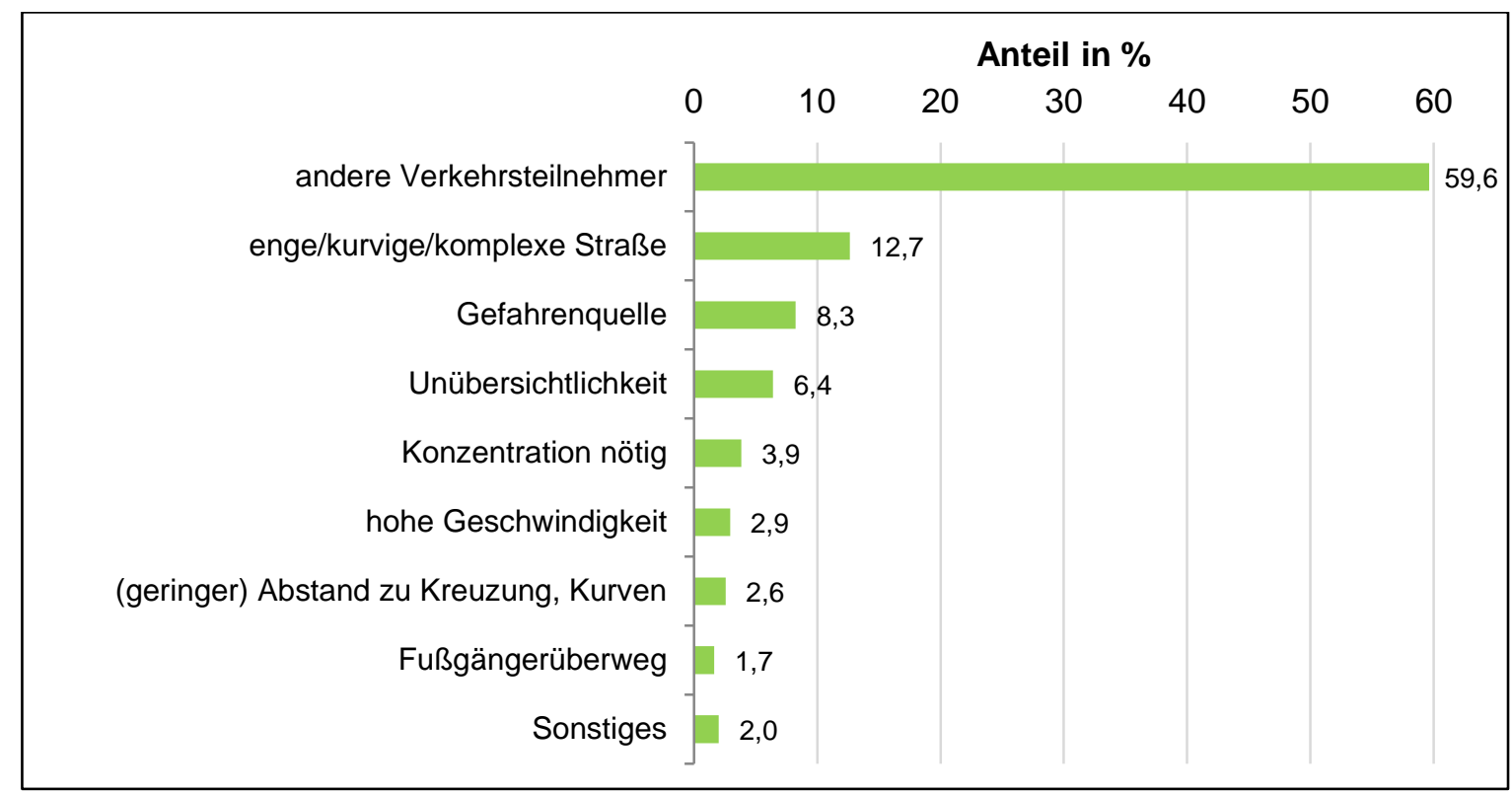

Abbildung 39: Nennungen (in \%) für Argumente was anders sein müsste, damit die Teilnehmer nicht mit dem Verfassen einer Textnachricht beginnen, Interviewdaten $(N=82)$.

Auf die Frage, was anders sein müsste, damit sie in der Situation beginnen würden eine Textnachricht zu verfassen, antworteten die Teilnehmer am häufigsten, dass sie beginnen würden, wenn sie eine breite gerade Strecke vorfänden (siehe Abbildung 40), z.B. "gerade Strecke ohne Abbiegung". Weitere benannte Merkmale waren das Fehlen anderer Verkehrsteilnehmer, das Warten an einer roten Ampel, oder allgemein eine hohe Übersichtlichkeit der Situation.

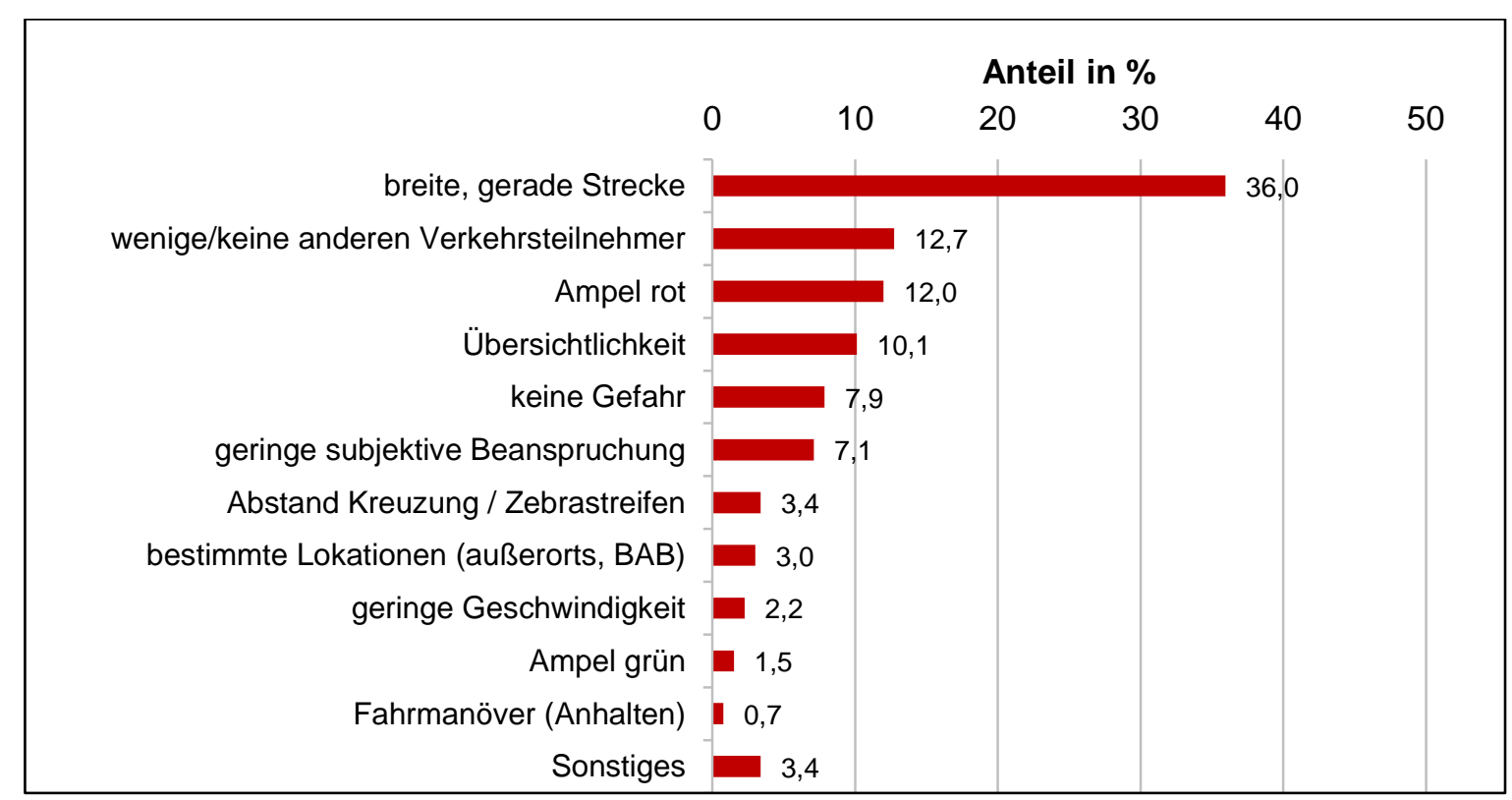

Abbildung 40: Nennungen (in \%) für Argumente was anders sein müsste, damit die Teilnehmer mit dem Verfassen einer Textnachricht beginnen, Interviewdaten $(N=82)$. 


\subsection{Zusammenfassung}

Zielstellung der beschriebenen Fahrsimulatorstudie war es zu untersuchen, inwieweit sich die selbstgewählte Zuwendung zu einer Nebentätigkeit auf das allgemeine Fahrverhalten und das Reaktionsverhalten in potentiell kritischen Verkehrssituationen auswirkt.

Beim allgemeinen Fahrverhalten ließen sich teilweise Effekte der Nebentätigkeitsbearbeitung identifizieren. So waren bei Streckenabschnitten mit einer Nebentätigkeit im Vergleich zu Streckenabschnitten ohne Nebenaufgabe zum Teil verringerte Geschwindigkeiten zu beobachten, was Befunden aus anderen Studien entspricht (z. B. Yager, 2013; Yannis et al., 2014). Auch zeigte sich eine geringe Variation der Spurposition, wenn die Teilnehmer eine Nebentätigkeit ausführten. Dies ist vor allem vor dem Hintergrund früherer Befunden zu den Folgen des visuell-manuellen Verfassens von Textnachrichten (Yager, Cooper \& Chrysler, 2012; He et al., 2015) überraschend. Gleichzeitig zeigen Untersuchungen zum Telefonieren beim Fahren, dass es besonders auf ruhigen Streckenabschnitten, ähnlich wie in der Fahrsimulatorstudie, durchaus zur Verringerung der Spurabweichungen kommen kann (Brookhuis, De Vries \& De Waard, 1991; Törnros \& Bolling, 2005). Allerdings ist generell einschränkend anzumerken, dass die beschriebenen Effekte auf Geschwindigkeit und Spurposition für Streckenabschnitte mit $50 \mathrm{~km} / \mathrm{h}$ Geschwindigkeitsbegrenzung nicht zu finden waren.

Auch in Bezug auf die potentiell sicherheitskritischen Situationen zeigten sich uneinheitliche Befunde. So wurden in der Baustellensituation keine (Beinahe-) Zusammenstöße registriert. Gleichzeitig kam es aber in der Situation, in der ein Kind auf die Straße lief, in knapp 40\% der Situationen zu einem Unfall oder Beinaheunfall. In diesem Zusammenhang war vor allem das Schreiben von Textnachrichten am Mobiltelefon auffällig, wie auch bei Drews (2009) bzw. Yannis und Kollegen (2014). Dabei wurden die meisten Unfälle und auch Beinaheunfälle registriert, während es zwischen den anderen Nebenaufgaben, auch im Vergleich zum Fahren ohne Nebentätigkeit, kaum Unterschiede gab. Hinsichtlich weiterer Fahrverhaltensindikatoren, wie etwa wann die Reduktion des Gaspedaldrucks oder die Bremspedalbetätigung erfolgte, wurden allerdings, anders als in früheren Arbeiten (Libby, Chaparro \& He, 2013; Yannis et al., 2016), keine systematischen Unterschiede zwischen den verschiedenen Nebentätigkeiten sowie dem Fahren ohne Nebentätigkeit gefunden. Tendenziell zeigte sich jedoch auch in diesen Maßen, dass die Teilnehmer beim Schreiben einer Nachricht etwas schlechter abschnitten als bei den anderen Nebenaufgaben. Beispielsweise, zeigte sich bei den Teilnehmern die Textnachrichten schrieben ein geringerer Abstand bzw. kleinere Time to Collision zum Kind beim Einsetzen der Bremsung. Die Vorinformation (Schild vor Baustelle bzw. Ball auf Straße), die den Fahrern die Möglichkeit geben sollte sich die potentiell kritische Situation zu antizipieren hatte den erwarteten positiven Effekt. So wurde durchweg deutlich früher auf das Ereignis reagiert, wenn die Vorinformation vorhanden war. Entsprechend weniger Unfälle und Beinaheunfälle waren auch zu beobachten. Darüber hinaus gab es aber keine Interaktionen zwischen der Präsenz der Vorinformation und der Art der Nebenaufgabe in den relevanten Fahrverhaltensparametern.

Bei den Situationen, in denen auf eine veränderte Geschwindigkeitsbegrenzung reagiert werden sollte, wurden ebenso keine systematischen Unterschiede zwischen den Nebentätigkeiten sowie dem Fahren ohne Nebenaufgabe beim Zeitpunkt der Geschwindigkeitsreduktion gefunden. Allerdings zeigte sich bei der parkähnlichen Situation, bei der das Schild sehr spät zu sehen war und eher abrupt reagiert werden 
musste, dass beim Schreiben einer Nachricht später reagiert wurde als ohne Nebenaufgabe.

Während das Schreiben von Textnachrichten in einigen Maßen eher auffällige Ergebnisse zur Folge hatte, sind die Befunde zur sprachbasierten Interaktion weitestgehend unauffällig. Frühere Untersuchungen ließen vermuten, dass die sprachbasierte Bedienung (Vorlesen, Spracheingabe) mit besseren Fahrleistungen als das Schreiben von Textnachrichten einhergehen würde, aber gleichzeitig dennoch Leistungseinbußen im Vergleich zum Fahren ohne Nebentätigkeit zu beobachten sein müssten ( $\mathrm{He}$ et al., 2014, 2015). Die vorliegende Fahrsimulatorstudie ergab diesbezüglich jedoch keine klaren Ergebnisse. So waren die Fahrleistungen mit sprachbasiertem System nicht systematisch verschieden von denen beim Lesen einer Textnachricht. Gleichzeitig fanden sich aber auch keine klaren Unterschiede im Verhalten zum Fahren ohne Nebenaufgabe. Ganz generell ist erstaunlich, dass sich das Fahrverhalten ohne Nebenaufgabe nicht wesentlich vom Fahrverhalten bei Bearbeitung der meisten Nebenaufgaben unterschied (mit Ausnahme des Schreibens von Textnachrichten). 


\section{Diskussion und Fazit}

Die Zielstellung dieses Projektes bestand darin zu prüfen, inwieweit Fahrer in vermeintlich sicheren, einfachen Verkehrssituationen, in denen sie sich mit einer hohen Wahrscheinlichkeit für die Bearbeitung einer Nebentätigkeit entscheiden, noch adäquat auf Hinweisreize und kritische Situationen reagieren können. In einem ersten Schritt sollten dazu zunächst die Kontexte identifiziert werden, in denen die Fahrer bereit sind Textnachrichten zu verfassen oder zu lesen. Hierfür wurde eine umfassende Literaturrecherche, eine Re-Analyse der naturalistischen Fahrstudie (SHRP2) als auch eine videobasierte Interviewstudie durchgeführt. Die Ergebnisse der verschiedenen Untersuchungen zeigen, dass situative Merkmale einen starken Einfluss darauf haben, ob Fahrer bereit sind, Textnachrichten während des Fahrens zu verfassen oder zu lesen. Entscheidend in den Situationen schienen übergeordnete Merkmale wie die Komplexität oder Vorhersehbarkeit zu sein. Wenn die Situation vorhersehbar schien bzw. eine geringe Komplexität aufwies (z.B. gerade Strecke, wenig Verkehr), waren die Fahrer durchaus bereit, eine Textnachricht zu schreiben oder zu lesen. Die Strecke der darauf aufbauenden Fahrsimulatorstudie wurde so umgesetzt, dass sie entsprechend dieser Merkmale vorhersehbar und von geringer Komplexität war. In der Untersuchung sollte dann getestet werden, wie sich die selbstgewählte Bearbeitung verschiedener Nebentätigkeiten auf das Fahr- und Reaktionsverhalten auswirkt.

Die Ergebnisse der Fahrsimulatorstudie lassen darauf schließen, dass die Fahrer ihr Fahrverhalten in einem gewissen Maße an die Bearbeitung der Nebentätigkeiten anpassen, z.B. in dem sie die Geschwindigkeit reduzieren. Der Fokus der Untersuchung lag allerdings auf möglichen Unterschieden im Reaktionsverhalten in verschiedenen Situationen zwischen den einzelnen Nebentätigkeiten und zum Fahren ohne Nebenaufgabe. Bei den meisten Maßen für das Reaktionsverhalten (wie Unfälle, Zeit bis zur Reduktion des Gaspedaldrucks oder Betätigung des Bremspedals, sowie Abstand / Time to Collision bei Reaktion) wurden keine systematischen Unterschiede zwischen den Nebentätigkeiten gefunden. Zudem ergaben sich auch keine Unterschiede im Vergleich zum Fahren ohne Nebentätigkeit. Lediglich beim visuell-manuellen Verfassen von Textnachrichten wurden bei einigen Maßen Beeinträchtigungen festgestellt. So zeigten sich beim Schreiben tendenziell Verzögerungen in der Reaktion auf eine kritische Situation, sowie erhöhte Raten an Unfällen bzw. Beinaheunfällen.

Die Tatsache, dass keine Unterschiede zwischen dem Reaktionsverhalten ohne Nebenaufgabe und dem Verhalten bei Bearbeitung des überwiegenden Teils der Nebenaufgaben (mit Ausnahme der visuell-manuellen Texteingabe) gefunden werden konnten, ist relativ überraschend, geht man von früheren Befunden aus (z.B. Caird, Johnston et al., 2014; He et al., 2014; Yannis et al., 2016). Eine mögliche Erklärung kann in der relativen Einfachheit der Strecke gesucht werden, die, wie auch die Teilnehmer in der Nachbefragung bestätigten, nur wenig beanspruchend war. Zwar wurde die Strecke bewusst mit der Zielstellung einer geringen Beanspruchung gestaltet, jedoch ist denkbar, dass dies zu einer Unterforderung der Fahrer, die keine Nebentätigkeit ausführen mussten, geführt hat. Laut dem Yerkes-Dodson Gesetz (Vollrath \& Krems, 2011; Yerkes \& Dodson, 1908) besteht zwischen der von einer Aufgabe ausgehenden Beanspruchung und der Leistung in dieser Aufgabe ein umgekehrt U-förmiger Zusammenhang. Das bedeutet, dass die beste Leistung bei mittlerer Beanspruchung erbracht wird, während eine sowohl zu geringe als auch zu hohe Beanspruchung mit Leistungseinbußen verbunden sind. Die sehr geringe Beanspruchung der Strecke könnte sich beim Fahren ohne Nebentätigkeit entsprechend negativ ausgewirkt haben. Vor diesem Hintergrund ist erklärbar, dass die Fahrperformanz ohne Nebentätigkeit im Vergleich zu den Fahrern, die einer Nebentätigkeit nachgingen, nicht erkennbar besser war. 
Gleichzeitig konnten allerdings bereits Schömig und Kollegen (2015) zeigen, dass Fahrer Nebenaufgaben in wenig beanspruchenden Fahrsituationen anscheinend tatsächlich ohne große Leistungseinbußen ausführen können, besonders wenn sie sich frei für oder gegen die Bearbeitung einer Nebentätigkeit entscheiden können. Wenn ihnen die Fahrsituation geeignet erscheint, bearbeiten sie Nebenaufgaben, wenn nicht, dann unterbleibt die Bearbeitung. In Anbetracht der Tatsache, dass sich die Reaktionsfähigkeit der Teilnehmer bei Bearbeitung der Nebentätigkeit nicht von den Fahrern ohne Nebentätigkeit unterschied kann demnach auch als Beleg dafür gewertet werden, dass die von den Teilnehmern vorgenommene Situationseinschätzung den Anforderungen der Situation angemessen war. Entsprechend ergaben sich keine Beeinträchtigungen in den relevanten Parametern. Inwieweit diese Einschätzung allerdings auch in komplexeren Verkehrssituationen als den dargestellten zuverlässig funktioniert, kann mit den vorliegenden Daten nicht geklärt werden.

Diese Problematik wird durch die generell hohe Bereitschaft der Teilnehmer im Versuch Nebenaufgaben zu bearbeiten verdeutlicht. So entschlossen sich die Fahrer bei fast jeder Möglichkeit zur Bearbeitung der Nebenaufgabe, nur sehr wenige Möglichkeiten blieben ungenutzt. Dies ist als weiterer Beleg für die geringe Komplexität der Strecke zu werten. Augenscheinlich wurde den Teilnehmern die Entscheidung zugunsten der Nebenaufgabenbearbeitung zu einfach gemacht. In nachfolgenden Untersuchungen sollte entsprechend eine höhere Variabilität in den Streckencharakteristika und damit auch der Komplexität kreiert werden, so dass es Deckeneffekte bei der Bearbeitung vermieden werden, und die Fahrer in bestimmten Verkehrssituationen tatsächlich an potentielle Grenzbereiche herangeführt werden.

Dass das Texten in der vorliegenden Untersuchung zumeist keine negativen Konsequenzen nach sich zog, entspricht durchaus der Wahrnehmung der meisten Fahrer im realen Straßenverkehr. Das setzt eine für die Verkehrssicherheit ungünstige Lernerfahrung in Gang. Wenn die Fahrer während des Fahrens Textnachrichten lesen oder schreiben ohne dass es zu negativen Konsequenzen kommt, wird dies als Bestätigung dafür gewertet, dass das Texten möglicherweise nicht so gefährlich ist. Dies führt in der Folge zur Aufrechterhaltung dieses Verhaltens und irgendwann auch in Situationen in denen es eine Gefahr darstellt. Diese Lernerfahrung aufzubrechen ist eine der zentralen Herausforderungen für die Verkehrssicherheitskommunikation. 


\section{$7 \quad$ Literatur}

Alm, H. \& Nilsson, L. (1994). Changes in driver behaviour as a function of handsfree mobile phones - A simulator study. Accident Analysis and Prevention, 26 (4), S. 441451.

doi: 10.1016/0001-4575(94)90035-3.

Basacik, D., Reed, N. \& Robbins, R. (2011). Smartphone use while driving - a simulator study. London.

Bayer, J. B. \& Campbell, S. W. (2012). Texting while driving on automatic: Considering the frequency-independent side of habit. Computers in Human Behavior, 28 (6), S. 2083-2090. doi: 10.1016/j.chb.2012.06.012.

Bitkom. (2017). Smartphone - Markt: Konjunktur und Trends. Zugriff: am 10.8.17, https://www.bitkom.org/Presse/Anhaenge-an-PIs/2017/02-Februar/Bitkom-

Pressekonferenz-Smartphone-Markt-Konjunktur-und-Trends-22-02-2017-

Praesentation.pdf

Brookhuis, K. A., De Vries, G. \& De Waard, D. (1991). The effects of mobile telephoning on driving performance. Accident Analysis and Prevention, 23 (4), S. 309-316.

Bundesgesetzblatt Jahrgang 2017 Teil I Nr. 68 (2017). Dreiundfünfzigste Verordnung zur Änderung straßenverkehrsrechtlicher Vorschriften vom 6. Oktober 2017. Bonn, abgerufen am 28.12.2017

https://www.bgbl.de/xaver/bgbl/start.xav\#_bggb__\%2F\%2F*\%5B\%40attr_id\%3D\%27bg bl117s3549.pdf\%27\%5D_1514458847080).

Bundesministerium für Verkehr und digitale Infrastruktur \& Bundesministerium für Umwelt, Naturschutz, Bau und Reaktorsicherheit (2017). Verordnung zur Änderung straßenverkehrsrechtlicher Vorschriften. Berlin.

Caird, J. K., Johnston, K. A., Willness, C. R. \& Asbridge, M. (2014). The use of metaanalysis or research synthesis to combine driving simulation or naturalistic study results on driver distraction. Journal of Safety Research. National Safety Council, 49 (February), S. 91-96. doi: 10.1016/j.jsr.2014.02.013.

Caird, J. K., Johnston, K. A., Willness, C. R., Asbridge, M. \& Steel, P. (2014). A metaanalysis of the effects of texting on driving. Accident Analysis and Prevention, 71, S. 311-318. doi: 10.1016/j.aap.2014.06.005.

Campbell, K. L. (2012). The SHRP 2 Naturalistic Driving Study. Transportation Research News, S. 30-35.

Choudhary, P. \& Velaga, N. R. (2017). Modelling driver distraction effects due to mobile phone use on reaction time. Transportation Research Part C: Emerging Technologies, 77, S. 351-365. doi: 10.1016/j.trc.2017.02.007.

DEKRA e.V. (2014). 3 Prozent aller Autofahrer mit Handy am Ohr. Stuttgart.

Dingus, T. A., Guo, F., Lee, S., Antin, J. F., Perez, M., Buchanan-King, M. \& Hankey, J. (2016). Driver crash risk factors and prevalence evaluation using naturalistic driving data. in Proceedings of the National Academy of Sciences of the United States of America, S. 1-6. doi: 10.1073/pnas.1513271113.

Dingus, T. A., Hankey, J. M., Antin, J. F., Lee, S. E., Eichelberger, L., Stulce, K. E., McGraw, D., Perez, M. \& Stowe, L. (2015). Naturalistic driving study: Technical coordination and quality control. Washington D.C. doi: S2-S06-RW-1. 
Drews, F. A., Yazdani, H., Godfrey, C. N., Cooper, J. M. \& Strayer, D. L. (2009). Text messaging during simulated driving. Human Factors: The Journal of the Human Factors and Ergonomics Society, 51 (5), S. 762-770. doi: 10.1177/0018720809353319.

Fastenmeier, W. (1995). Taxonomien von Verkehrssituationen. In Häcker, H. (Hrsg.) Autofahrer und Verkehrssituation. Köln, Bonn: Verlag TÜV Rheinland GmbH, Deutscher Psychologen Verlag, S. 44-78.

forsa Politik- und Sozialforschung GmbH (2016). Nutzung von Smartphones bzw. Mobiltelefonen im Straßenverkehr. Berlin.

Harrison, M. A. (2011). College students' prevalence \& perceptions of text messaging while driving. Accident Analysis and Prevention, 43 (4), S. 1516-1520. doi: 10.1016/j.aap.2011.03.003.

He, J., Chaparro, A., Nguyen, B., Burge, R. J., Crandall, J., Chaparro, B. S., Ni, R. \& Cao, S. (2014). Texting while driving: Is speech-based text entry less risky than handheld text entry? Accident Analysis and Prevention, 72, S. 287-295. doi: 10.1016/j.aap.2014.07.014.

He, J., Choi, W., McCarley, J. S., Chaparro, B. S. \& Wang, C. (2015). Texting while driving using Google Glass: Promising but not distraction-free. Accident Analysis and Prevention, 81, S. 218-229. doi: 10.1016/j.aap.2015.03.033.

heise online. (2017). Endkundenabsatz von Smartphones weltweit von 2007 bis 2016 (in Millionen Stück). In Statista - Das Statistik-Portal. Zugriff am 25. Juli 2017, von https://de.statista.com/statistik/daten/studie/12856/umfrage/absatz-von-smartphonesweltweit-seit-2007/

Hosking, S. G., Young, K. L. \& Regan, M. A. (2009). The effects of text messaging on young drivers. Human Factors, 51 (4), S. 582-592. doi: 10.1177/0018720809341575.

Huth, V., Sanchez, Y. \& Brusque, C. (2014). Drivers' phone use at red traffic lights: A roadside observation study comparing calls and visual-manual interactions. Accident Analysis and Prevention, 74, S. 42-48. doi: 10.1016/j.aap.2014.10.008.

Kidd, D. G., Tison, J., Chaudhary, N. K., McCartt, A. T. \& Casanova-Powell, T. D. (2016). The influence of roadway situation, other contextual factors, and driver characteristics on the prevalence of driver secondary behaviors. Transportation Research Part F: Traffic Psychology and Behaviour, 41, S. 1-9. doi: 10.1016/j.trf.2016.06.004.

Klauer, S. G., Klauer, S. G., Dingus, T. a., Dingus, T. a., Neale, V. L., Neale, V. L., Sudweeks, J. D., Sudweeks, J. D., Ramsey, D. J. \& Ramsey, D. J. (2006). The Impact of driver inattention on near crash/crash risk: An analysis using the 100-Car naturalistic driving study data. doi: DOT HS 810594.

Kraftfahrtbundesamt (2017). Anzahl der registrierten Verstöße gegen das Handyverbot am Steuer in Deutschland von 2005 bis 2015. In Statista - Das Statistik-Portal. Zugriff am $25 . \quad$ Juli 2017, von https://de.statista.com/statistik/daten/studie/224044/umfrage/registrierte-verstoessegegen-das-handyverbot-am-steuer-seit-2005/.

Kröling, S. \& Gehlert, T. (2016) Verkehrsklima in Deutschland 2016 Unfallforschung kompakt. Berlin: Gesamtverband der Deutschen Versicherungswirtschaft e.V. Unfallforschung der Versicherer.

Kubitzki, J. \& Fastenmeier, W. (2016) Ablenkung durch moderne Informations-und Kommunikationstechniken und soziale Interaktion bei Autofahrern. Unterföhring: AZT Automotive $\mathrm{GmbH}$ - Allianz Zentrum für Technik \& Institut Mensch Verkehr Umwelt. 
Leung, S., Croft, R. J., Jackson, M. L., Howard, M. E. \& Mckenzie, R. J. (2012). A comparison of the effect of mobile phone use and alcohol consumption on driving simulation performance. Traffic Injury Prevention, 13 (6), S. 566-574. doi: 10.1080/15389588.2014.925299.

Libby, D., Chaparro, A. \& He, J. (2013). Distracted while driving: A comparison of the effects of texting and talking on a cell phone. In Proceedings of the Human Factors and Ergonomics Society Annual Meeting, S. 1874-1878. doi: 10.1177/1541931213571418.

Lipovac, K., Đerić, M., Tešić, M., Andrić, Z. \& Marić, B. (2017). Mobile phone use while driving-literary review. Transportation Research Part F: Traffic Psychology and Behaviour, 47, S. 132-142. doi: 10.1016/j.trf.2017.04.015.

Mayring, P. (2000). Qualitative Inhaltsanalyse. Grundlagen und Techniken (7. Auflage). Weinheim: Deutscher Studien Verlag.

McKeever, J. D., Schultheis, M. T., Padmanaban, V. \& Blasco, A. (2013). Driver performance while texting: even a little is too much. Traffic Injury Prevention, 14 (2), S. 132-137. doi: 10.1080/15389588.2012.699695.

Paridon, H., Hofmann, S. \& Schreiber, F. (2015). Manuelle versus sprachgesteuerte Bearbeitung von SMS während einer Autofahrt: Effekte auf Leistung, Beanspruchung und physiologische Parameter. Zeitschrift für Verkehrssicherheit, 61, S. 28-32.

Popken, A. (2009). Drivers' reliance on lane keeping assistance systems as a function of the level of assistance. Doctoral dissertation, Chemnitz University of Technology. Von: $<$ http://archiv.tu-chemnitz.de/pub/2010/0048/index.html>.

Prat, F., Gras, M. E., Planes, M., Font-Mayolas, S. \& Sullman, M. J. M. (2017). Driving distractions: An insight gained from roadside interviews on their prevalence and factors associated with driver distraction. Transportation Research Part F: Traffic Psychology and Behaviour, 45, S. 194-207. doi: 10.1016/j.trf.2016.12.001.

Rauch, N., Gradenegger, B. \& Krüger, H.-P. (2008) Das Konzept des Situationsbewusstseins und seine Implikationen für die Fahrsicherheit. Veitshöchheim.

Rauch, N., Gradenegger, B. \& Krüger, H.-P. (2009). Darf ich oder darf ich nicht? Situationsbewusstsein im Umgang mit Nebenaufgaben während der Fahrt. Zeitschrift für Arbeitswissenschaften, 1 (9), S. 3-17.

Rudin-Brown, C. M., Young, K. L., Patten, C., Lenné, M. G. \& Ceci, R. (2013) 'Driver distraction in an unusual environment: Effects of text-messaging in tunnels. Accident Analysis and Prevention, 50, S. 122-129. doi: 10.1016/j.aap.2012.04.002.

Schömig, N. \& Metz, B. (2013) Three levels of situation awareness in driving with secondary tasks. Safety Science, 56, S. 44-51. doi: 10.1016/j.ssci.2012.05.029.

Schömig, N., Schoch, S., Neukum, A., Schumacher, M. \& Wandtner, B. (2015) Simulatorstudien zur Ablenkungswirkung fahrfremder Tätigkeiten. Bergisch Gladbach: Bundesanstalt für Straßenwesen.

Simmons, S. M., Hicks, A. \& Caird, J. K. (2016). Safety-critical event risk associated with cell phone tasks as measured in naturalistic driving studies: A systematic review and meta-analysis. Accident Analysis and Prevention, 87, S. 161-169. doi: 10.1016/j.aap.2015.11.015.

Simons-Morton, B. G., Guo, F., Klauer, S. G., Ehsani, Johnathon, P. \& Pradhan, A. K. (2014). Keep your eyes on the road: young driver crash risk increases according to duration of distraction. The Journal of Adolescent Health, 54(5), S. S61-S67. doi: 10.1016/j.jadohealth.2013.11.021. 
Struckman-Johnson, C., Gaster, S., Struckman-Johnson, D., Johnson, M. \& MayShinagle, G. (2015). Gender differences in psychosocial predictors of texting while driving. Accident Analysis and Prevention, 74, S. 218-228. doi: 10.1016/j.aap.2014.10.001.

Tivesten, E. \& Dozza, M. (2014). Driving context and visual-manual phone tasks influence glance behavior in naturalistic driving. Transportation Research Part F: Traffic Psychology and Behaviour, 26, S. 258-272. doi: 10.1016/j.trf.2014.08.004.

Tivesten, E. \& Dozza, M. (2015). Driving context influences drivers' decision to engage in visual-manual phone tasks: Evidence from a naturalistic driving study. Journal of Safety Research, 53, S. 87-96. doi: 10.1016/j.jsr.2015.03.010.

Törnros, J. \& Bolling, A. (2006). Mobile phone use - effects of conversation on mental workload and driving speed in rural and urban environments. Transportation Research Part F: Traffic Psychology and Behaviour, 9(4), S. 298-306. doi: 10.1016/j.trf.2006.01.008.

Törnros, J. \& Bolling, A. (2005). Mobile phone use - Effects of handheld and handsfree phones on driving performance. Accident Analysis and Prevention, 37 (5), S. 902-909. doi: 10.1016/j.aap.2005.04.007.

Virginia Tech Transportation Institute (2015) Researcher Dictionary for Video Reduction Data. Blacksburg, Virginia.

Vollrath, M., Huemer, A. K., Nowak, P., Pion, O. \& Hummel, T. (2014) Ablenkung durch Informations- und Kommunikationssysteme Ablenkung durch Informations- und Kommunikationssysteme. Berlin: Gesamtverband der Deutschen Versicherungswirtschaft e. V. Unfallforschung der Versicherer.

Vollrath, M., Huemer, A. K., Teller, C., Likhacheva, A. \& Fricke, J. (2016) 'Do German drivers use their smartphones safely? - Not really!. Accident Analysis and Prevention, 96, S. 29-38. doi: 10.1016/j.aap.2016.06.003.

Vollrath, M. \& Krems, J. (2011) Verkehrspsychologie: Ein Lehrbuch für Psychologen, Ingenieure und Informatiker. Kohlhammer.

Xiong, H., Bao, S., Sayer, J. \& Kato, K. (2015) 'Examination of drivers' cell phone use behavior at intersections by using naturalistic driving data. Journal of Safety Research, 54 (June), p. 89.e29-93. doi: 10.1016/j.jsr.2015.06.012.

Yager, C. E. (2013a). An evaluation of the effectiveness of voice-to-text programs at reducing incidences of distracted driving. Austin, Texas.

Yager, C. E. (2013b). Driver safety impacts of voice-to-text mobile applications. in Proceedings of the Human Factors and Ergonomics Society Annual Meeting. San Diego, S. 1869-1873. doi: 10.1177/1541931213571417.

Yager, C. E., Cooper, J. M. \& Chrysler, S. T. (2012). The effects of reading and writing text-based messages while driving. In Proceedings of the Human Factors and Ergonomics Society Annual Meeting, S. 2196-2200. doi: 10.1177/1071181312561463.

Yannis, G., Laiou, A., Papantoniou, P. \& Christoforou, C. (2014). Impact of texting on young drivers' behavior and safety on urban and rural roads through a simulation experiment. Journal of Safety Research, 49 (February), S. 25-31. doi: 10.1016/j.jsr.2014.02.008.

Yannis, G., Laiou, A., Papantoniou, P. \& Gkartzonikas, C. (2016). Simulation of texting impact on young drivers behavior and safety on motorways. Transportation Research Part F: Traffic Psychology and Behaviour, 41, S. 10-18. doi: 10.1016/j.trf.2016.06.003. 
Yerkes, R.M., \& Dodson, J.D. (1908). The relation of strength of stimulus to rapidity of habit-formation. Journal of Comparative Neurology and Psychology, 18, 459-482.

Young, K. L. \& Lenné, M. G. (2010). Driver engagement in distracting activities and the strategies used to minimise risk. Safety Science, 48 (3), S. 326-332. doi: 10.1016/j.ssci.2009.10.008.

Zijlstra, F. R. H. (1985) The Construction of a Scale to Measure Perceived Effort.

Zijlstra, F. R. H. (1993) Efficiency in work behaviour: A design approach for modern tools. Technische Universiteit Delft. doi: 90-6275-918-1. 


\section{Anhang}

Interviewleitfaden

Situation 1: [beschreiben]

01 Würden Sie in der gezeigten Situationen mit dem $\square$ Ja Schreiben einer Textnachricht beginnen?

Falls JA: Warum würden Sie in dieser Situation mit dem Schreiben beginnen?

02

Falls NEIN: Warum würden Sie in dieser Situation nicht mit dem Schreiben beginnen?

[Bitte darauf hinweisen, dass nur Merkmale der gezeigten Situationen relevant sind und nicht globale Dinge wie: „wenn mir jemand schreibt".]

Falls JA: Was müsste in der Situation anders sein, damit Sie nicht beginnen? (nur 1 Merkmal)

03 Falls NEIN: Was müsste in der Situation anders sein, damit Sie beginnen? (nur 1 Merkmal)

[bei globalen Antworten wie "Stau“ nachfragen, was müsste direkt in der gerade gezeigten Situation anders sein?]

\section{Risikobewertung Situation 1}

Stellen Sie sich vor, Sie würden in dieser Situation eine Textnachricht verfassen (ungeachtet der zuvor gegebenen Antwort) - um wie viel Prozent wäre Ihrer Meinung nach die Wahrscheinlichkeit eines Unfalls erhöht? 


\section{GDV \\ DIE DEUTSCHEN VERSICHERER}

Gesamtverband der Deutschen Versicherungswirtschaft e. V.

Wilhelmstraße 43 / 43G, 10117 Berlin

Postfach 0802 64, 10002 Berlin

Telefon 030 / 2020 - 50 00, Fax 030 / 2020 - 6000

Internet: www.gdv.de, www.udv.de 